\title{
Embedded Sensors and Controls to Improve Component Performance and Reliability Conceptual Design Report
}

\section{September 2012}

\author{
Prepared by \\ R. Kisner \\ A. Melin, \\ T. Burress, \\ D. Fugate, \\ D. Holcomb, \\ J. Wilgen, \\ J. Miller, \\ D. Wilson, \\ P. Silva, \\ L. Whitlow, and \\ F. Peretz
}

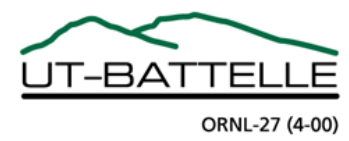




\title{
DOCUMENT AVAILABILITY
}

Reports produced after January 1, 1996, are generally available free via the U.S. Department of Energy (DOE) Information Bridge.

Web site http://www.osti.gov/bridge

Reports produced before January 1, 1996, may be purchased by members of the public from the following source.

\author{
National Technical Information Service \\ 5285 Port Royal Road \\ Springfield, VA 22161 \\ Telephone 703-605-6000 (1-800-553-6847) \\ TDD 703-487-4639 \\ Fax 703-605-6900 \\ E-mail info@ntis.gov \\ Web site http://www.ntis.gov/support/ordernowabout.htm
}

Reports are available to DOE employees, DOE contractors, Energy Technology Data Exchange (ETDE) representatives, and International Nuclear Information System (INIS) representatives from the following source.

Office of Scientific and Technical Information

P.O. Box 62

Oak Ridge, TN 37831

Telephone 865-576-8401

Fax 865-576-5728

E-mail reports@osti.gov

Web site http://www.osti.gov/contact.html

This report was prepared as an account of work sponsored by an agency of the United States Government. Neither the United States Government nor any agency thereof, nor any of their employees, makes any warranty, express or implied, or assumes any legal liability or responsibility for the accuracy, completeness, or usefulness of any information, apparatus, product, or process disclosed, or represents that its use would not infringe privately owned rights. Reference herein to any specific commercial product, process, or service by trade name, trademark, manufacturer, or otherwise, does not necessarily constitute or imply its endorsement, recommendation, or favoring by the United States Government or any agency thereof. The views and opinions of authors expressed herein do not necessarily state or reflect those of the United States Government or any agency thereof. 
ORNL/TM-2012/433

Measurement Science and Systems Engineering Division

\title{
EMBEDDED SENSORS AND CONTROLS TO IMPROVE COMPONENT PERFORMANCE AND RELIABILITY CONCEPTUAL DESIGN REPORT
}

\author{
R. Kisner \\ A. Melin, \\ T. Burress, \\ D. Fugate, \\ D. Holcomb, \\ J. Wilgen, \\ J. Miller, \\ D. Wilson, \\ P. Silva, \\ L. Whitlow, and \\ F. Peretz
}

Date Published: September 2012

\author{
Prepared by \\ OAK RIDGE NATIONAL LABORATORY \\ Oak Ridge, Tennessee 37831-6283 \\ managed by \\ UT-BATTELLE, LLC \\ for the \\ U.S. DEPARTMENT OF ENERGY \\ under contract DE-AC05-00OR22725
}





\section{CONTENTS}

PIST OF FIGURES
LIST OF TABLES
ABBREVIATED TERMS
EXECUTIVE SUMMARY
ABSTRACT T
1.3

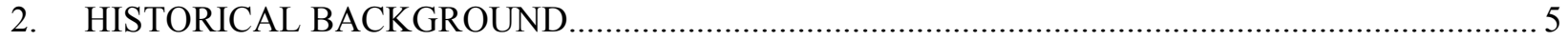

2.1 ADVANCES ENABLED BY EMBEDDED SENSORS \& CONTROLS ….......................... 6

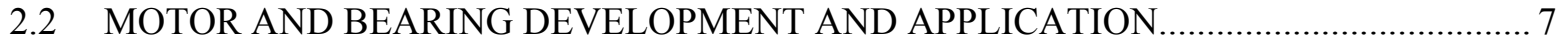

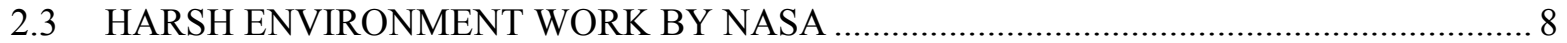

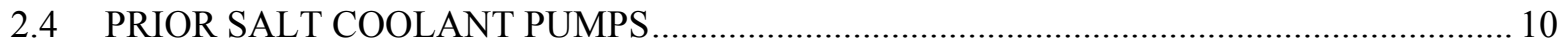

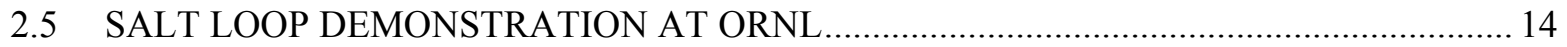

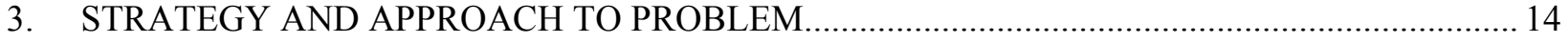

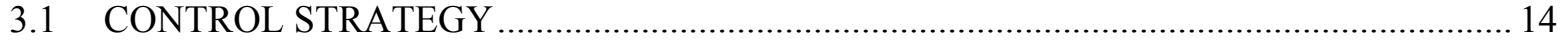

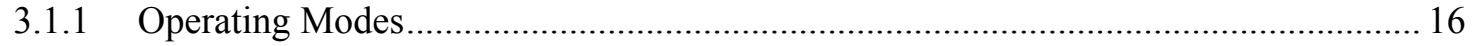

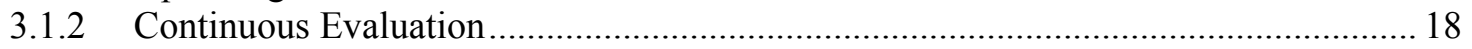

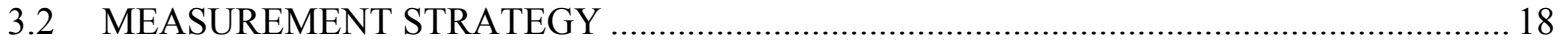

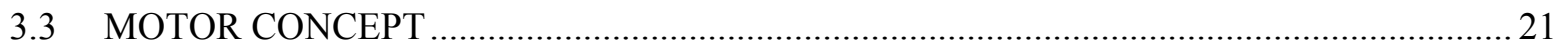

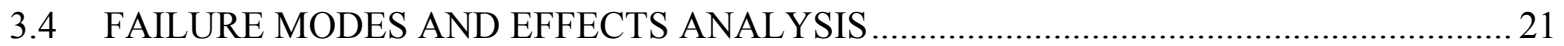

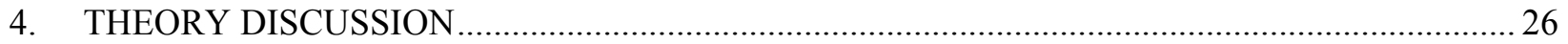

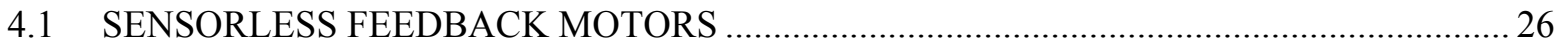

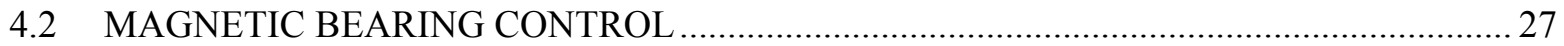

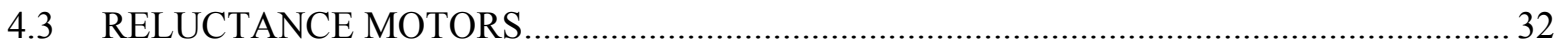

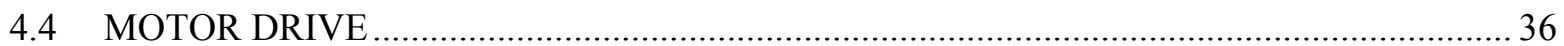

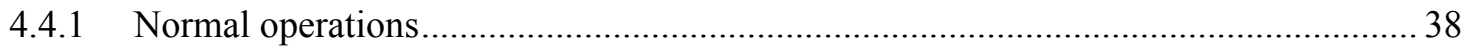

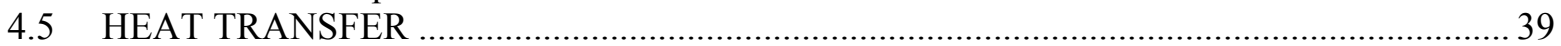

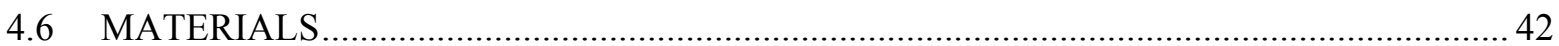

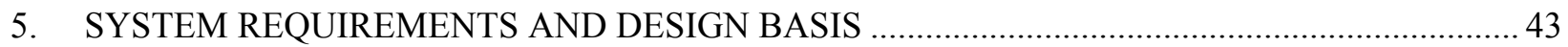

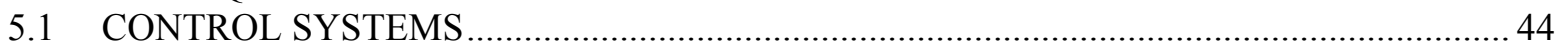

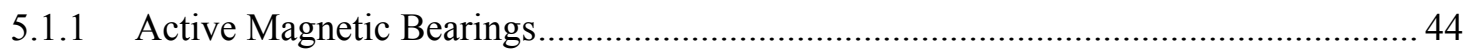

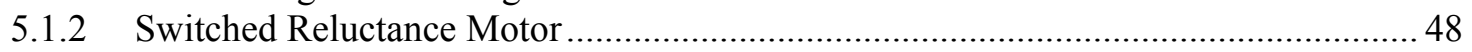

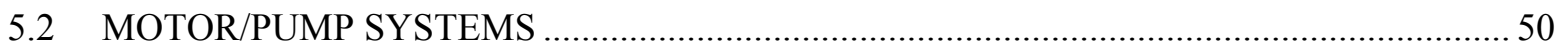

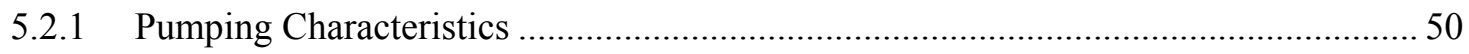

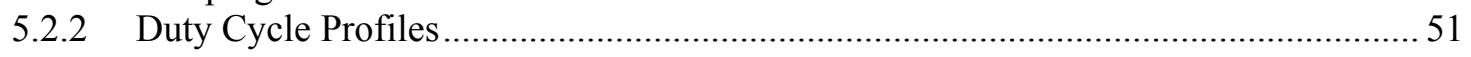

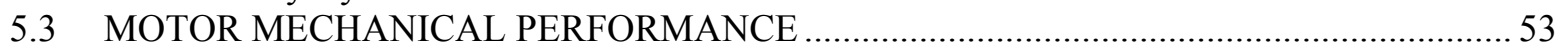

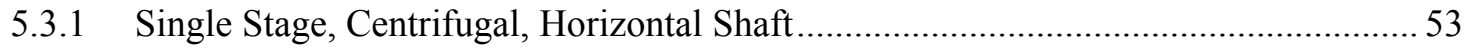

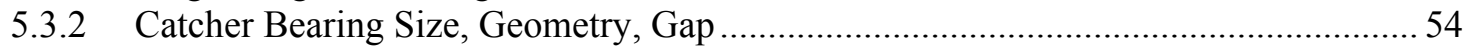

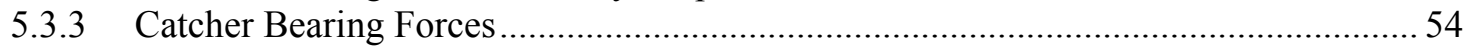

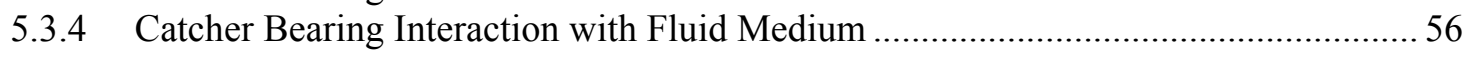

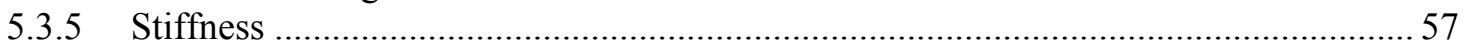

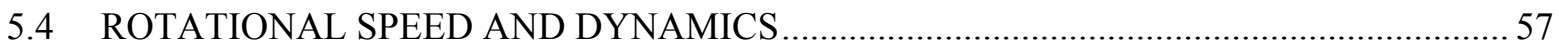




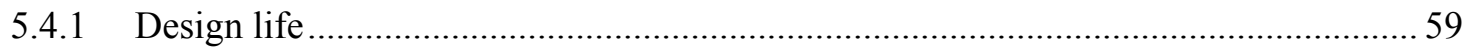

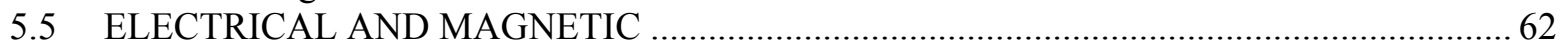

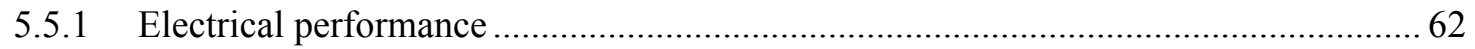

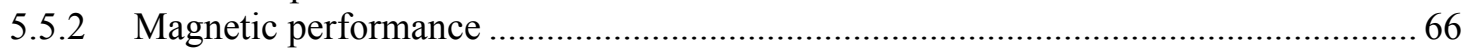

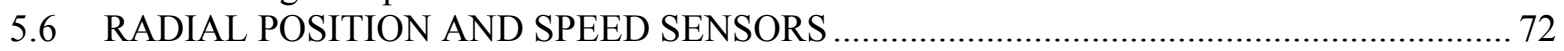

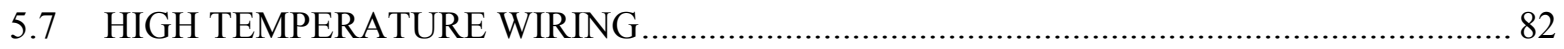

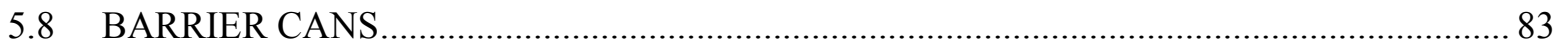

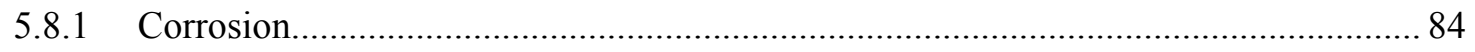

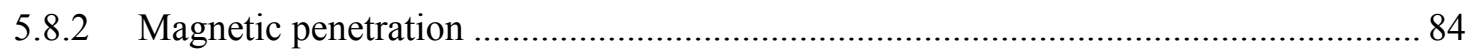

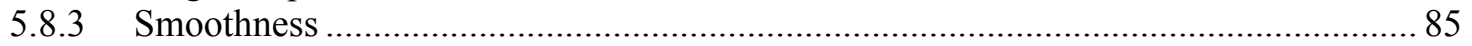

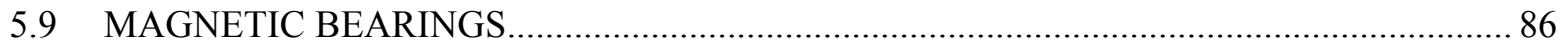

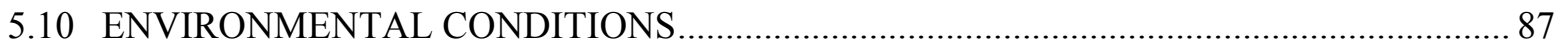

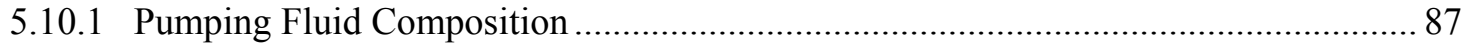

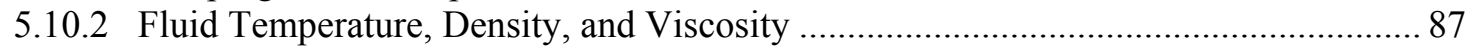

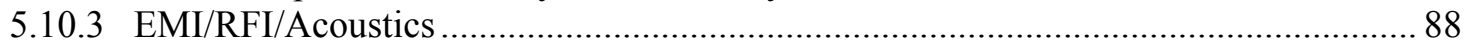

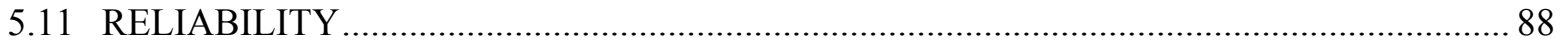

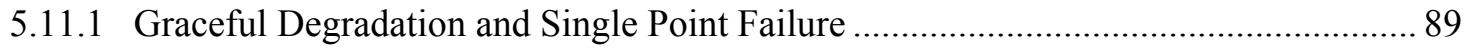

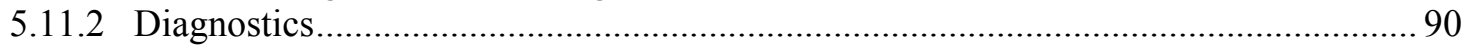

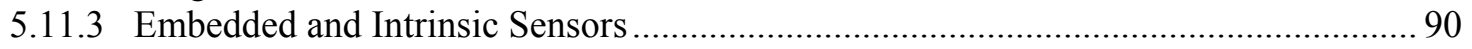

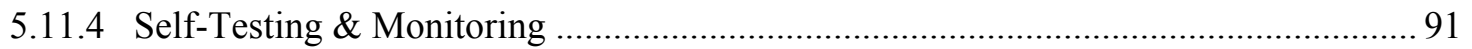

5.11.5 Prognostics and Anticipated Time to Failure ........................................................ 92

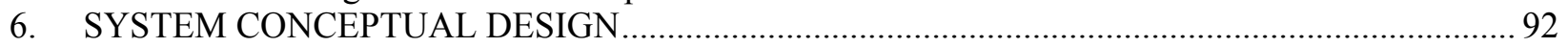

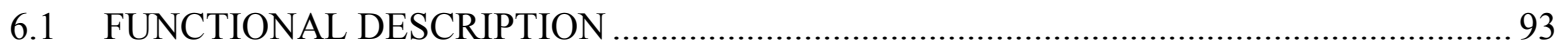

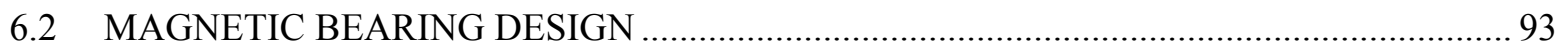

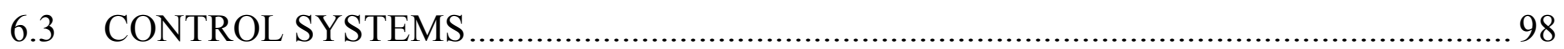

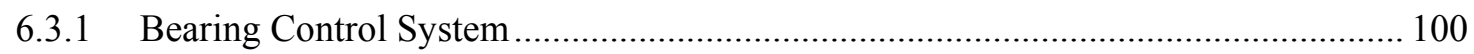

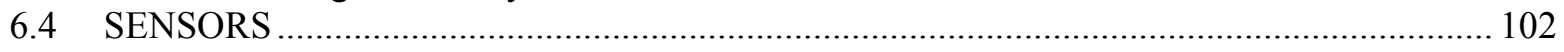

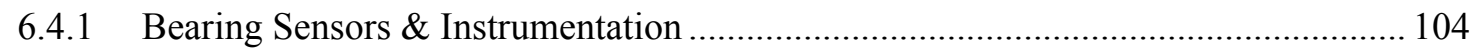

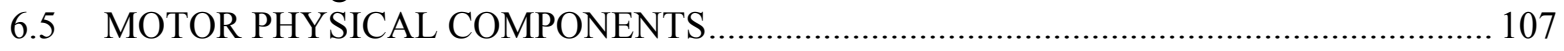

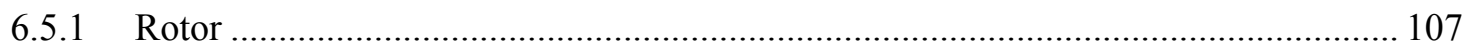

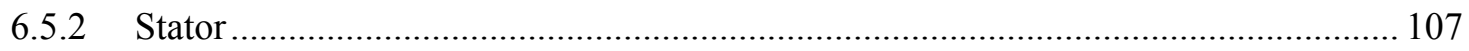

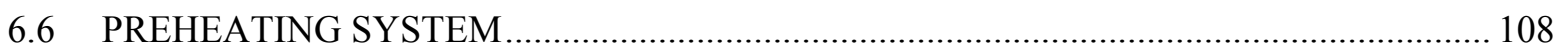

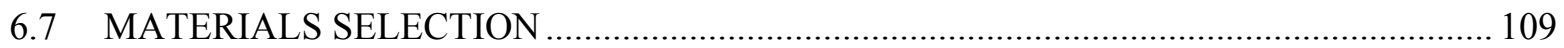

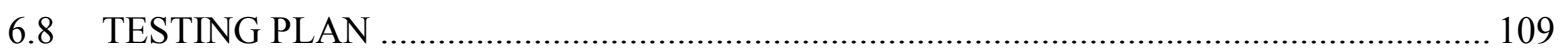

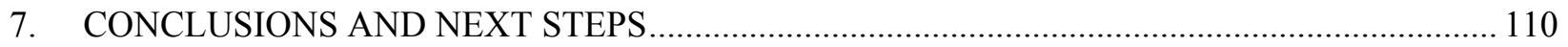

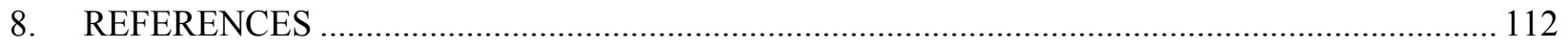

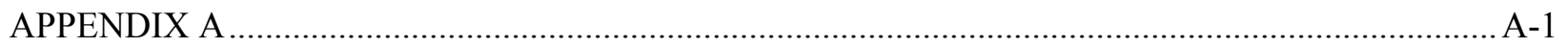




\section{LIST OF FIGURES}

Figure $\quad$ Page

Fig. 1. Systems engineering integrates all involved disciplines into the design process. ......................... 3

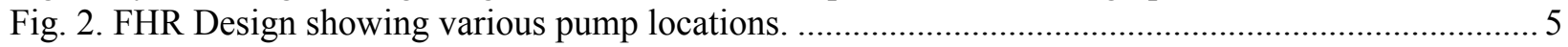

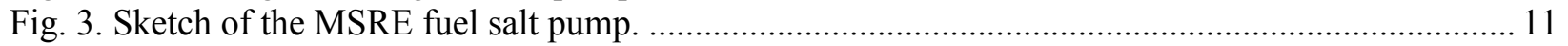

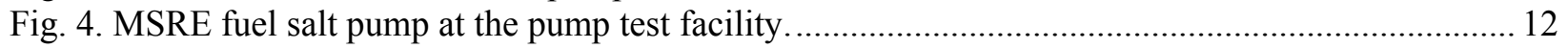

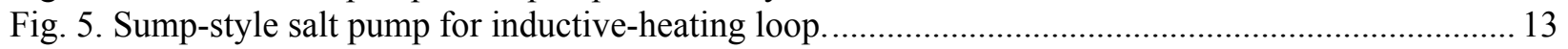

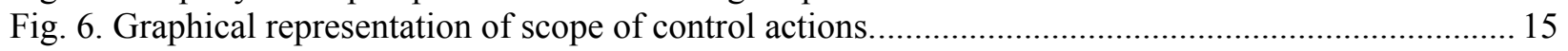

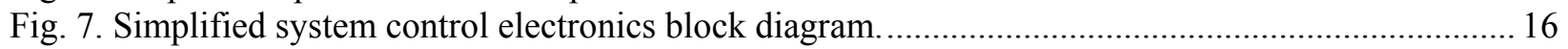

Fig. 8. Reference illustration of canned rotor motor with pump impeller. ............................................... 19

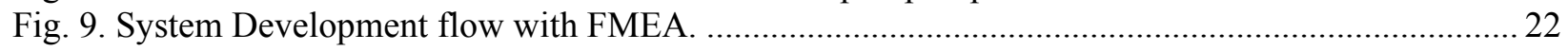

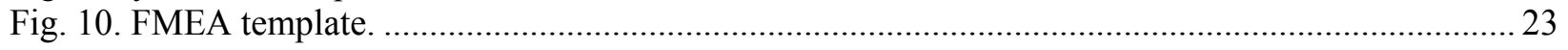

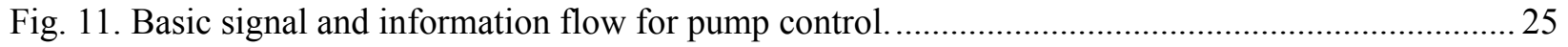

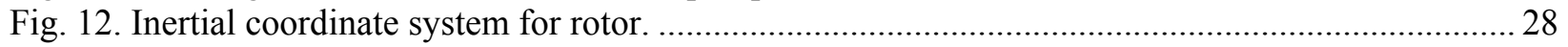

Fig. 13. Simulation showing the rotor force with a 10 A coil current and varying position from -0.6

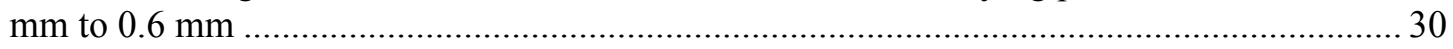

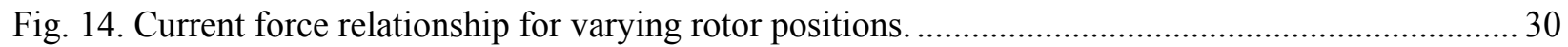

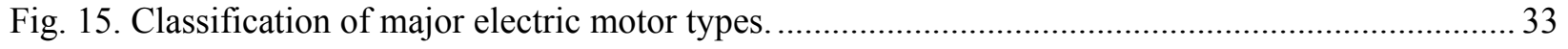

Fig. 16. Electric motors shown in cross section by major type............................................................. 34

Fig. 17. The 6/4 SRM baseline geometry and associated power electronics stage .................................. 37

Fig. 18. Representative $6 / 4$ SRM phase current waveforms at $\mathrm{n} \sim 312 \mathrm{rpm}$ with phase turn-on angle

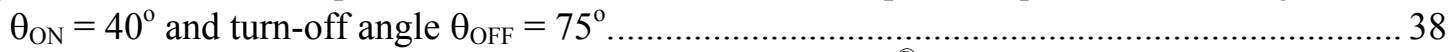

Fig. 19. Representative 6/4 SRM. Left: stator assembly with Hastelloy ${ }^{\circledR}$ can. Right: rotor assembly

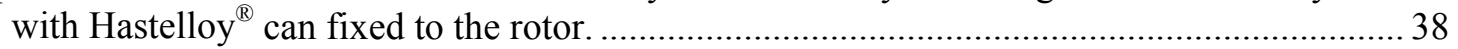

Fig. 20. Representative 6/4 SRM with rotor and stator conducting can and resultant torque

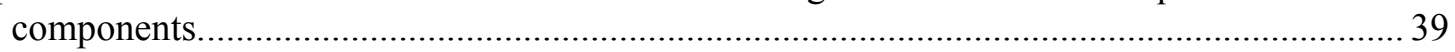

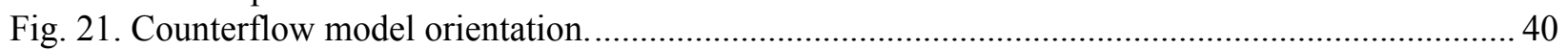

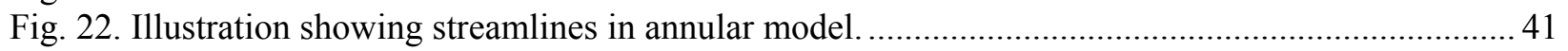

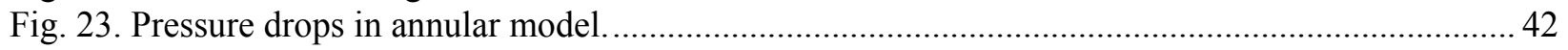

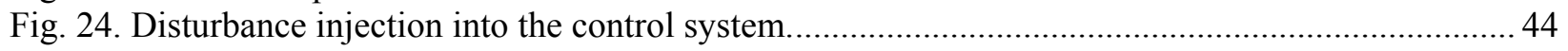

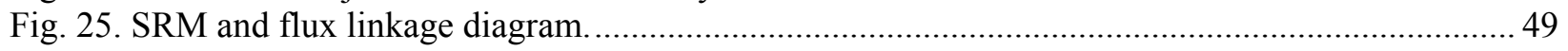

Fig. 26. Switched reluctance motor speed control block diagram. ................................................ 50

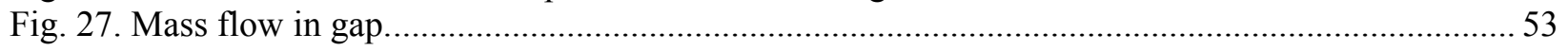

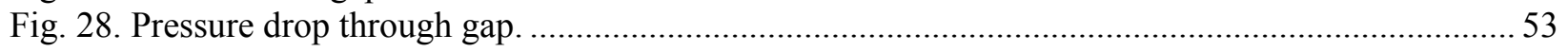

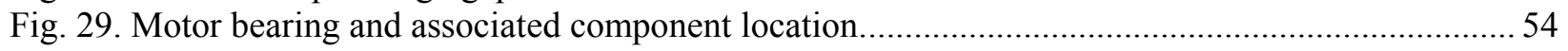

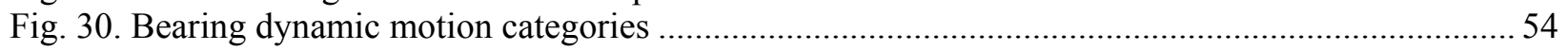

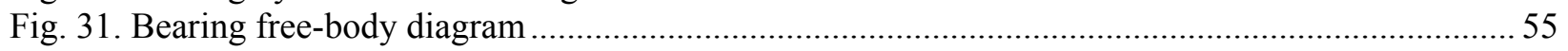

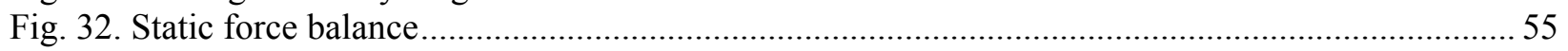

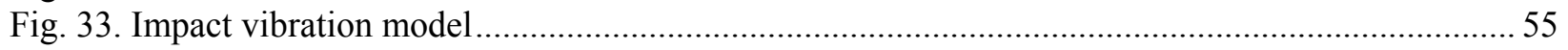

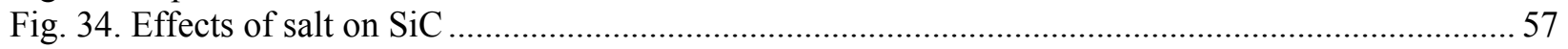

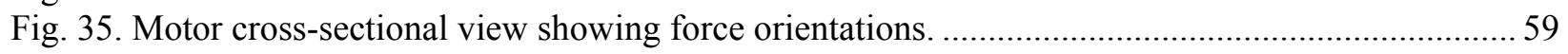

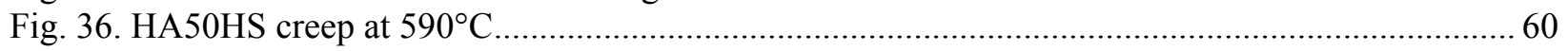

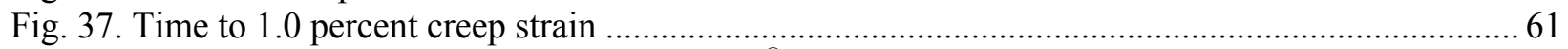

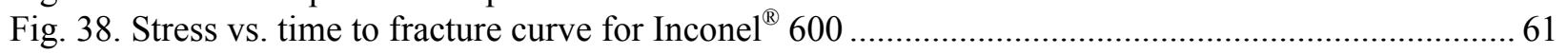

Fig. 39. SRM energy conversion for large gap design. Center: 6/4 SRM aligned, Right: Unaligned........ 63

Fig. 40. SRM inductance variation (A-phase solid trace) and representative phase-A current pulse......... 66

Fig. 41. SRM phase power pulses for rectangular current input when $\mathrm{n}=3600 \mathrm{rpm}$...........................6 66 
Fig. 42. Top Left: SRM simulated torque vs rotor angle, $\theta_{\mathrm{r}}=0^{\circ}$ in fully unaligned position and $\theta_{\mathrm{r}}=$ $180^{\circ}$ electrical in fully aligned position relative to phase A (as shown). Top right: $\mathrm{m}\left(\theta, \mathrm{I}_{\mathrm{s}}\right)$ when $\mathrm{g}=1 \mathrm{~mm}$, bottom left: $\mathrm{m}\left(\theta, \mathrm{I}_{\mathrm{s}}\right)$ when $\mathrm{g}=3 \mathrm{~mm}$, and bottom right: $\mathrm{m}_{\mathrm{pk}}\left(\theta, \mathrm{I}_{\mathrm{s}}\right)$ for $\mathrm{g}=1 \mathrm{~mm}$ vs. $\mathrm{g}=3 \mathrm{~mm}$ case.

Fig. 43. Left: SRM simulated torque/amp for $\mathrm{g}=1 \mathrm{~mm}$; right: torque/amp for $\mathrm{g}=3 \mathrm{~mm}$.................. 68

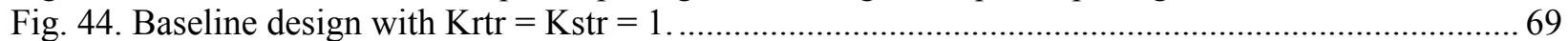

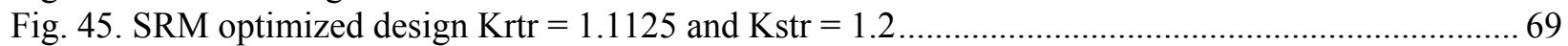

Fig. 46. SRM locked rotor torque evaluation when $\mathrm{Krtr}=1$, $\mathrm{Kstr}=1.05$, and $\mathrm{g}=1 \mathrm{~mm} \ldots \ldots \ldots \ldots \ldots \ldots \ldots \ldots . . . \ldots 9$

Fig. 47. SRM average locked rotor torque at $\mathrm{Is}=200 \mathrm{~A}$ and $\mathrm{g}=1 \mathrm{~mm}$ over spread of Krtr and Kstr....... 70

Fig. 48. SRM average locked rotor torque at $\mathrm{Is}=200 \mathrm{~A}$ and $\mathrm{g}=3 \mathrm{~mm}$ over spread of Krtr and Kstr....... 70

Fig. 49. Tooth widths for maximum average locked rotor torque for $\mathrm{g}=1 \mathrm{~mm}$ and $3 \mathrm{~mm}$................... 71

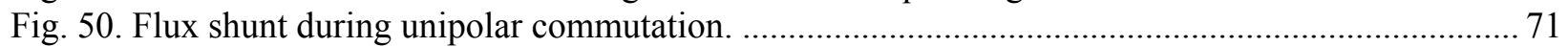

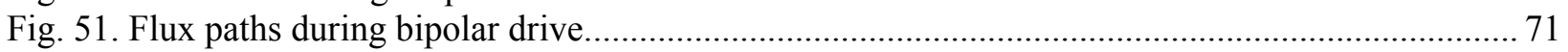

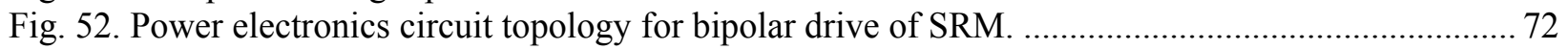

Fig. 53. Toyota Prius motor resolver (left) and Hyundai Sonata motor resolver (right).......................... 74

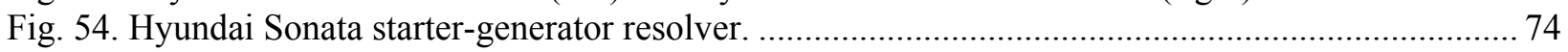

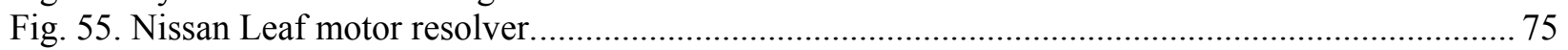

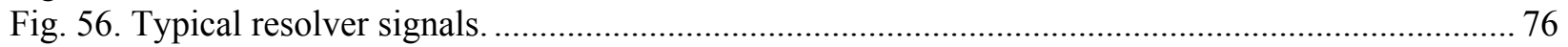

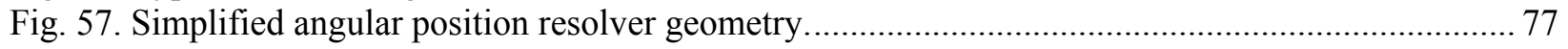

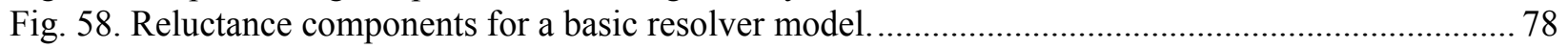

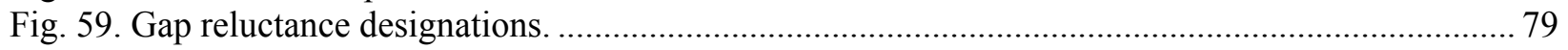

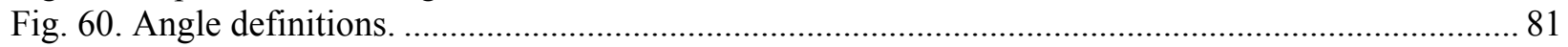

Fig. 61. Sine signals with $\mathrm{K}_{\text {ellipse }}$ values of 0.9 (top), 0.95 (middle), and 0.97 (bottom)........................ 81

Fig. 62. Angular measurement error with Kellipse values of 0.9 (top), 0.95 (middle), and 0.97

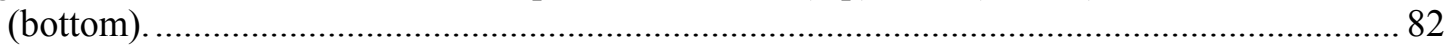

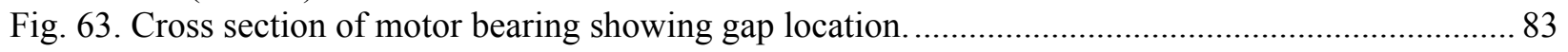

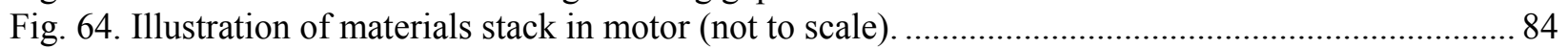

Fig. 65. Characteristic frequency response as a function of thickness.................................................. 85

Fig. 66. Characteristic frequency response as a function of aspect ratio............................................. 85

Fig. 67. 3D rendering of motor concept showing axial and radial bearings and main torque drive

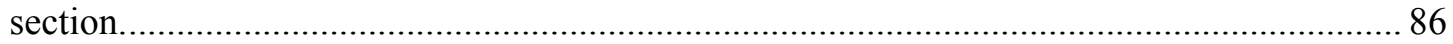

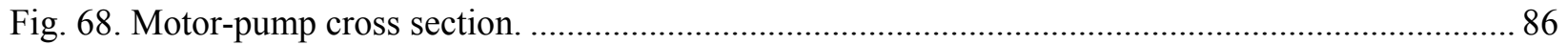

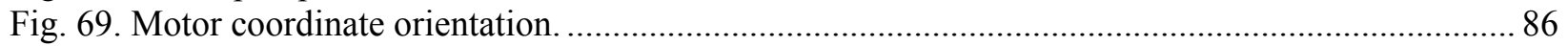

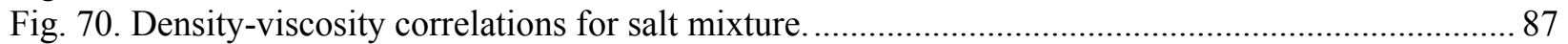

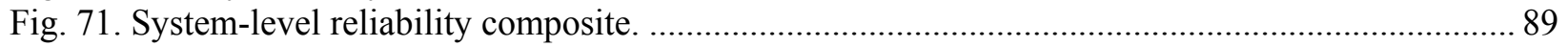

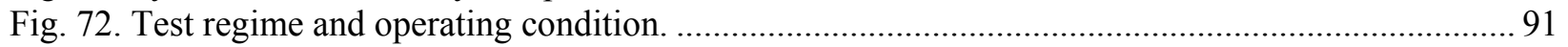

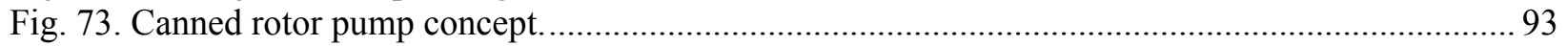

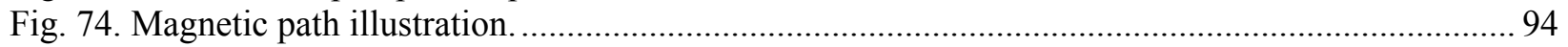

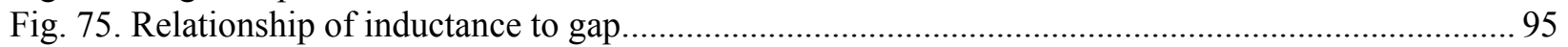

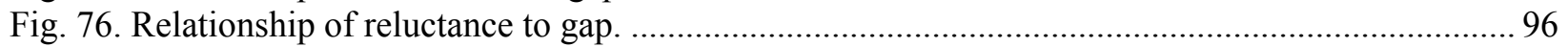

Fig. 77. Required number of turns vs. input current for constant force and 1-mm gap.......................... 97

Fig. 78. Required number of turns for constant input current over a fluid gap range............................. 97

Fig. 79. Required number of turns for a range of electromagnetic forces at three different input

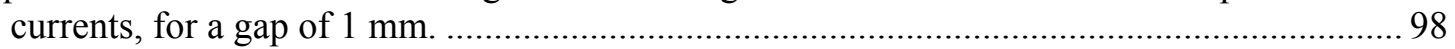

Fig. 80. Required current to provide electromagnetic force for a given number of coils $(\mathrm{N})$ and to

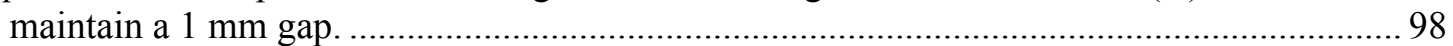

Fig. 81. Control and signal-processing components of embedded motor system summarized at a

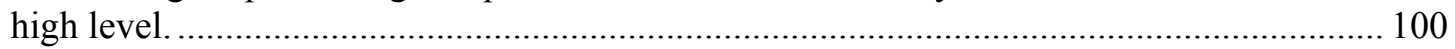

Fig. 82. Switched reluctance motor speed control block diagram showing loop gain stages. ................... 100

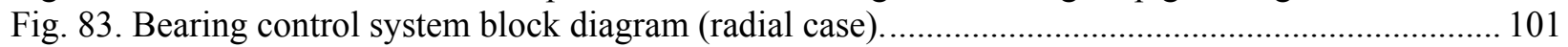

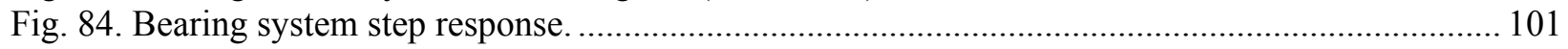


Fig. 85. Closed loop gain and phase estimations for bearing control. ................................................ 102

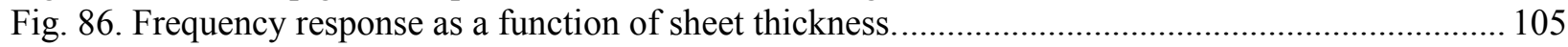

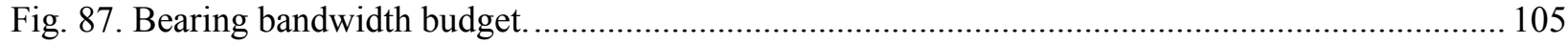

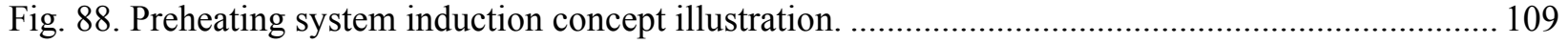

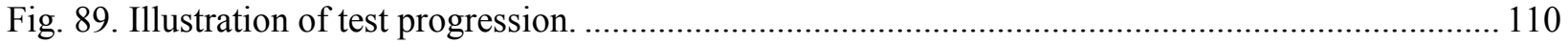





\section{LIST OF TABLES}

\section{Table}

Page

Table 1. Example set of requirements that empower the systems engineering process........................... 2

Table 2. Needed measurement signals for specific motor system component.......................................... 19

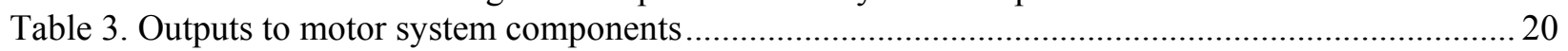

Table 4. Canned rotor motor subsystem breakdown related to FMEA .................................................... 24

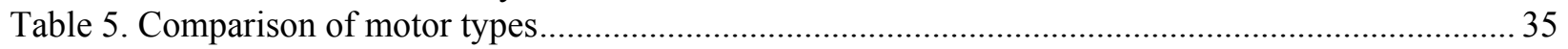

Table 6. Comparison of three motor types relative to high-temperature applications.............................. 36

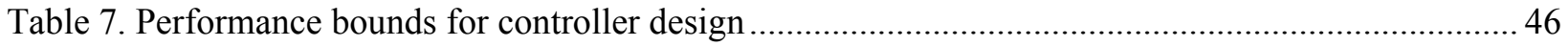

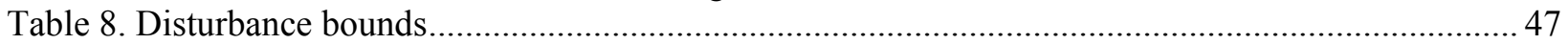

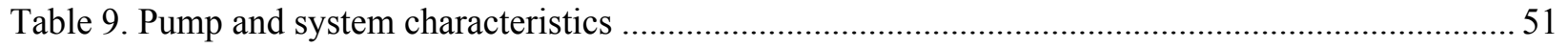

Table 10. Silicon Carbide bearing parameters. CRC Materials Science and Engineering Handbook ........ 57

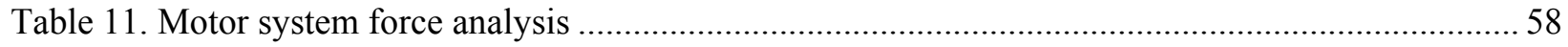

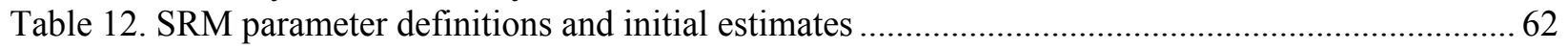

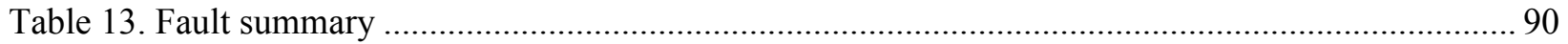

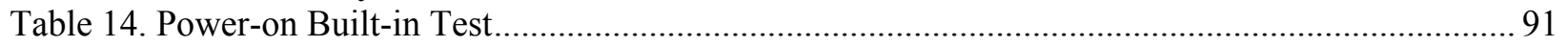

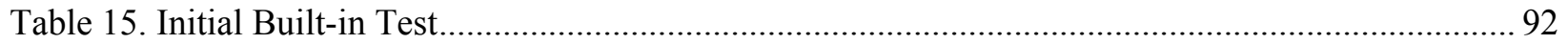

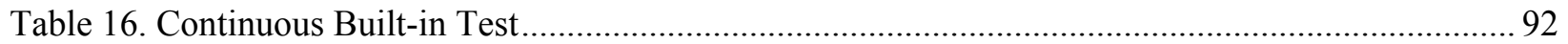

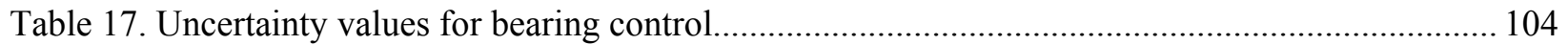

Table 18. List of processes requiring input currents and associated frequencies .................................. 106 



\section{ABBREVIATED TERMS}

\begin{tabular}{|c|c|}
\hline $\mathrm{A} / \mathrm{D}$ & analog to digital \\
\hline $\mathrm{ac}$ & alternating current \\
\hline AHTR & Advanced High Temperature Reactor \\
\hline AWG & American Wire Gauge \\
\hline CBIT & continuous built-in test \\
\hline $\mathrm{CD}$ & compact disk \\
\hline $\mathrm{CMF}$ & core melt frequency \\
\hline $\mathrm{dc}$ & direct current \\
\hline DCS & distributed control system \\
\hline DFBW & digital fly-by-wire \\
\hline DMC & dynamic matrix control \\
\hline DOE & US Department of Energy \\
\hline DOE-NE & US Department of Energy Office of Nuclear Energy \\
\hline DVD & digital versatile disk \\
\hline EDM & electrical discharge machining \\
\hline EMF & electromotive force \\
\hline EMI & electromagnetic interference \\
\hline FEA & finite-element analysis \\
\hline FHR & fluoride-salt-cooled high-temperature reactor \\
\hline FLiBe & $\mathrm{LiF}-\mathrm{BeF}_{2}$ \\
\hline FLiNaK & eutectic LiF-NaF-KF salt mixture \\
\hline FMEA & failure modes and effects analysis \\
\hline FPGA & field programmable gate array \\
\hline HTR & high-temperature reactor \\
\hline $\mathrm{HV}$ & high voltage \\
\hline $\mathrm{I} \& \mathrm{C}$ & Instrumentation and controls \\
\hline IBIT & initial built-in test \\
\hline IC & integrated circuit \\
\hline ID & inner diameter \\
\hline IGBT & insulated-gate bipolar transistor \\
\hline IM & inertial moment \\
\hline LED & light-emitting diode \\
\hline LQG & linear quadratic Gaussian \\
\hline
\end{tabular}


LRF large release frequency

LWR light-water reactors

MIMO multi-input/multi-output

MOSFET metal-oxide-semiconductor field-effect transistor

MPC model predictive control

MSBR Molten Salt Breeder Reactor

MSRE Molten Salt Reactor Experiment

MTBF mean-time-between-failure

NASA National Aeronautics and Space Administration

NEET Nuclear Energy Enabling Technology

NPP nuclear power plant

NRC US Nuclear Regulatory Commission

OD outer diameter

ORNL Oak Ridge National Laboratory

PBIT power-on built-in test

PD proportional-derivative controller

$\mathrm{PF} \quad$ power factor

PID proportional-integral-derivative controller

PM permanent magnet

PWM pulse-width modulated

RF radio frequency

rps rotor position sensing

$\mathrm{SiC} \quad$ silicon carbide

$\mathrm{SOH} \quad$ state-of-health

SRM switched reluctance motor

SSC systems, structures, and components

TC thermocouple

UAV unmanned aerial vehicle

VFD variable frequency drive

$\mathrm{ZOH} \quad$ zero-order hold 


\section{EXECUTIVE SUMMARY}

This project, sponsored by the US Department of Energy (DOE) Nuclear Energy Enabling Technology (NEET) program, focuses on the development of advanced embedded sensors and controls to achieve significant performance, reliability, and safety improvements for nuclear reactor components and systems. As survivability and reliability are critical, designing and validating embedded measurement and control technologies in representative operating conditions are needed. With this framework in mind, a canned rotor pump platform is being constructed as a validation platform for advanced sensor and control development. The demonstration platform is a suitable application in advanced reactor designs that present challenging environments and operational demands. Consequently, the benefits from enhanced control system capabilities can be clearly assessed while the project outcome will also fulfill a need for improved technology to meet the goals of advanced reactor performance. Additionally, the complexity of the system is such that traditional control techniques are inadequate and more advanced techniques need to be utilized for the system to be feasible.

The cost of nuclear energy is directly related to the cost and reliability of nuclear power plant (NPP) components. Deeply embedding instrumentation and controls (I\&C) within these components has the potential to significantly increase the reliability of the components while enabling otherwise unobtainable performance and reduced maintenance cost. Directly measuring changes in component characteristics and performance can obviate a significant portion of component maintenance, which is both time-consuming and expensive in itself and, more importantly, lowers plant availability. Embedding I\&C within components is key to observing degradation in the component and predicting remaining useful life. Additionally, enabling active response to stressors can reduce component cost by avoiding overdesign. Modern control electronics now have sufficient speed and reliability to provide dynamic control of highspeed mechanical components such as pump and turbine shafts. Seals and mechanical bearings are especially problematic as unanticipated bearing and seal failures have been the root cause of significant accidents. These situations largely could have been avoided by designs employing modern, embedded $\mathrm{I} \& \mathrm{C}$.

The objective of this project is to explore the potential gains from advanced embedded I\&C. Hightemperature pumps represent a particularly suitable target demonstration due to their large potential for performance and reliability improvement and their widespread usage in advanced NPPs. Historically, coolant pumps contribute to high plant reliability but pose an ongoing maintenance burden due mainly to seals and bearings. The practical objective of this work is to develop and demonstrate sensors and controls that operate as embedded parts of a high-temperature, canned rotor pump with magnetic bearings. This level of demonstration provides a practical example to the nuclear industry that inspires confidence in the design and use of components that embed sensors and controls as an intimate part of their functionality.

Because the I\&C is intimately part of the basic millisecond-by-millisecond functioning of the system, the I\&C design cannot be done in isolation from the other aspects of the system. The system cannot achieve the performance, efficiency, and robustness if the features of the I\&C are not included throughout the design. The I\&C enables optimal and reliable performance and tolerance of noise and uncertainties in the system rather than merely monitoring quasi-stable performance. Integrating $\mathrm{I} \& \mathrm{C}$ within the design to monitor and control dynamic behavior of components and systems is a new paradigm for the nuclear power industry.

Developing a testing platform suitable for use in high-temperature reactors (HTRs) is also supportive of Department of Energy Office of Nuclear Energy (DOE-NE). The demonstration platform will be designed for fluoride salt cooled high temperature reactors (FHRs), but the technology developed in this project will cross-cut multiple HTR designs. FHRs are a candidate advanced reactor class that has the potential to 
be economically preferable to light-water reactors (LWRs) while maintaining full passive safety. Additionally, a pumped liquid-fluoride-salt test loop has recently been constructed at ORNL along with a vertical shaft pump. This project will take advantage of the contemporaneous development both as a demonstration platform and as a comparison to a more traditional pump design.

The initial phase of the project was an assessment of the state of the art across the suite of disciplines required to construct a suitable testing environment to validate our embedded I\&C concepts. The evaluation included high temperatures, embedded sensing, fault tolerant controls, salt-compatible materials, high-speed power electronics, and modeling and simulation tools. A preconceptual design was then developed to evaluate the reasonableness of the overall concept.

Once basic conceptual viability was established, an initial conceptual design was created by first developing thermo-mechanical-electrical models of the test platform and then integrating the models into a common simulation framework. Performance characteristics and requirements were then identified using both analytic and computational models. Means to identify and characterize off-normal performance were then identified by combining performance requirements with potential failure mechanisms and available measurement technologies.

All of the building block technologies for creating a salt-compatible testing platform are generally known. The project has now produced an initial design and has not identified any basic flaws or unachievable material performance issues. However, the combined requirements for high reliability, graceful degradation, and long-term, high-temperature operation create a complex coupled control system. To handle this complexity, both detailed simulation and experimental validation are required to translate an early-phase conceptual design into a working demonstration platform for I\&C validation. For example, vibrational modes of the shaft will affect the position measurement system, control algorithm, power electronics, and the magnetic elements.

NPPs are high-capital-cost items necessitating very high component reliability. Shutting down a power plant due to subcomponent failure is financially unacceptable. System reliability is thus a central metric to the pump system design. Entropy requires that all components degrade over time. To minimize the potential for component failure during operation, NPPs employ extensive (and expensive) maintenance programs. The pump control system must, therefore, be designed to gracefully degrade in the event of unplanned subcomponent failures and allow for on-line exterior subcomponent replacement. Further, the embedded measurements must identify impending failures to allow any necessary maintenance to be performed during planned plant shutdowns. 


\begin{abstract}
The objective of this project is to demonstrate improved reliability and increased performance made possible by deeply embedding instrumentation and controls (I\&C) in nuclear power plant (NPP) components and systems. The project is employing a highly instrumented canned rotor, magnetic bearing, fluoride salt pump as its $I \& C$ technology demonstration platform. $I \& C$ is intimately part of the basic millisecond-by-millisecond functioning of the system; treating I\&C as an integral part of the system design is innovative and will allow significant improvement in capabilities and performance. As systems become more complex and greater performance is required, traditional I\&C design techniques become inadequate and more advanced I\&C needs to be applied. New I\&C techniques enable optimal and reliable performance and tolerance of noise and uncertainties in the system rather than merely monitoring quasistable performance. Traditionally, I\&C has been incorporated in NPP components after the design is nearly complete; adequate performance was obtained through over-design. By incorporating I\&C at the beginning of the design phase, the control system can provide superior performance and reliability and enable designs that are otherwise impossible. This report describes the progress and status of the project and provides a conceptual design overview for the platform to demonstrate the performance and reliability improvements enabled by advanced embedded I\&C.
\end{abstract}

\title{
1. INTRODUCTION
}

Embedding modern sensors and controls deeply within the systems, structures, and components (SSC) of nuclear power plants (NPPs) has the potential for otherwise unattainable improvements in performance, reliability, and reduced maintenance cost. The innovative approach of integrating the I\&C design into the system engineering will allow more advance control systems to be deployed. High-temperature pumps represent a particularly suitable target demonstration due to their large potential for performance and reliability improvement and their widespread usage in advanced NPPs. Advanced embedded I\&C can increase coolant pump reliability and reduce their maintenance burden on the plant by minimizing the need for seals and removing mechanical bearings. The practical objective of this work is to develop and demonstrate sensors and controls that operate as embedded parts of a high-temperature, canned rotor pump with magnetic bearings. This demonstration offers to the nuclear industry a concrete example to inspire confidence in the design and deployment of components with embedded sensors and controls as a part of their functionality.

Feedback control systems rely on the sensor measurement for operation, and the dynamics of the controller and sensor are intimately coupled. The high-temperature, harsh environments of advanced reactors - gas, liquid metal, and liquid-salt-cooled provide a challenging environment for sensor design, and the increased noise and uncertainty in the measurements necessary for feedback control mean that traditional control techniques do not provide sufficient performance and stability for these highly complex multidisciplinary integrated systems. The new sensor techniques and high degree of coupling in the system necessitates the implementation of advanced control techniques capable of stabilizing the system and maintaining the performance requirements and physical bounds necessary for safe long-term operation. This report describes the conceptual design of a demonstration platform for the development and validation of advanced embedded I\&C concepts and sensors. This report is intended to describe results of the research work conducted in fiscal year 2012 and to serve as a reference document throughout the project. The project is sponsored by the US Department of Energy (DOE) Nuclear Energy Enabling Technology (NEET) program whose mission is to develop crosscutting technologies that directly support and complement the Office of Nuclear Energy's (NE) development of new and advanced reactor concepts and fuel cycle technologies. 


\subsection{EMBEDDING AND SYSTEMS ENGINEERING BACKGROUND}

Embedding modern sensors and controls deeply within the SSC of NPPs has the potential to attain a new level of performance, reliability, and maintenance cost reductions. The successful embedding of sensors and controls in a complex working component is more than pursuing "add-on" features; rather, it is part of the larger effort of systems engineering, which is a process. Systems engineering is an interdisciplinary approach focusing on how complex engineering projects should be designed and managed over their life cycles. ${ }^{1}$ The goal of systems engineering is to produce systems that meet or exceed all mission requirements within schedule and budget. The influence of systems engineering on producing effective systems is highest during the conceptual design stages of a project.

The systems engineering approach relies on defined requirements that the design consistently references from early conceptual stages to the end of life cycle. Example requirements such as performance and reliability are illustrated in Table 1 . These requirements drive subsequent evaluation, analysis, and synthesis iteration loops throughout the life cycle. The multidisciplinary nature of systems engineering and the embedded sensors and control approach is expressed in Fig. 1. In the engineering design process, it is difficult to focus solely on one discipline to the exclusion of the others; rather, the process is holistic because a change in one area ripples through the design and affects many others. It is important to include the controller design in this process because sensors, controllers, and system design are interrelated.

Table 1. Example set of requirements that empower the systems engineering process

\begin{tabular}{ll}
\hline \multicolumn{1}{c}{ Requirements } & \multicolumn{1}{c}{ Subrequirements } \\
\hline Technical & $\begin{array}{l}\text { Functional } \\
\text { Performance } \\
\text { Interface }\end{array}$ \\
Operational & Robustness \\
Reliability & Fault tolerance \\
& Diversity/redundancy \\
Safety & \\
Maintainability & \\
\hline
\end{tabular}




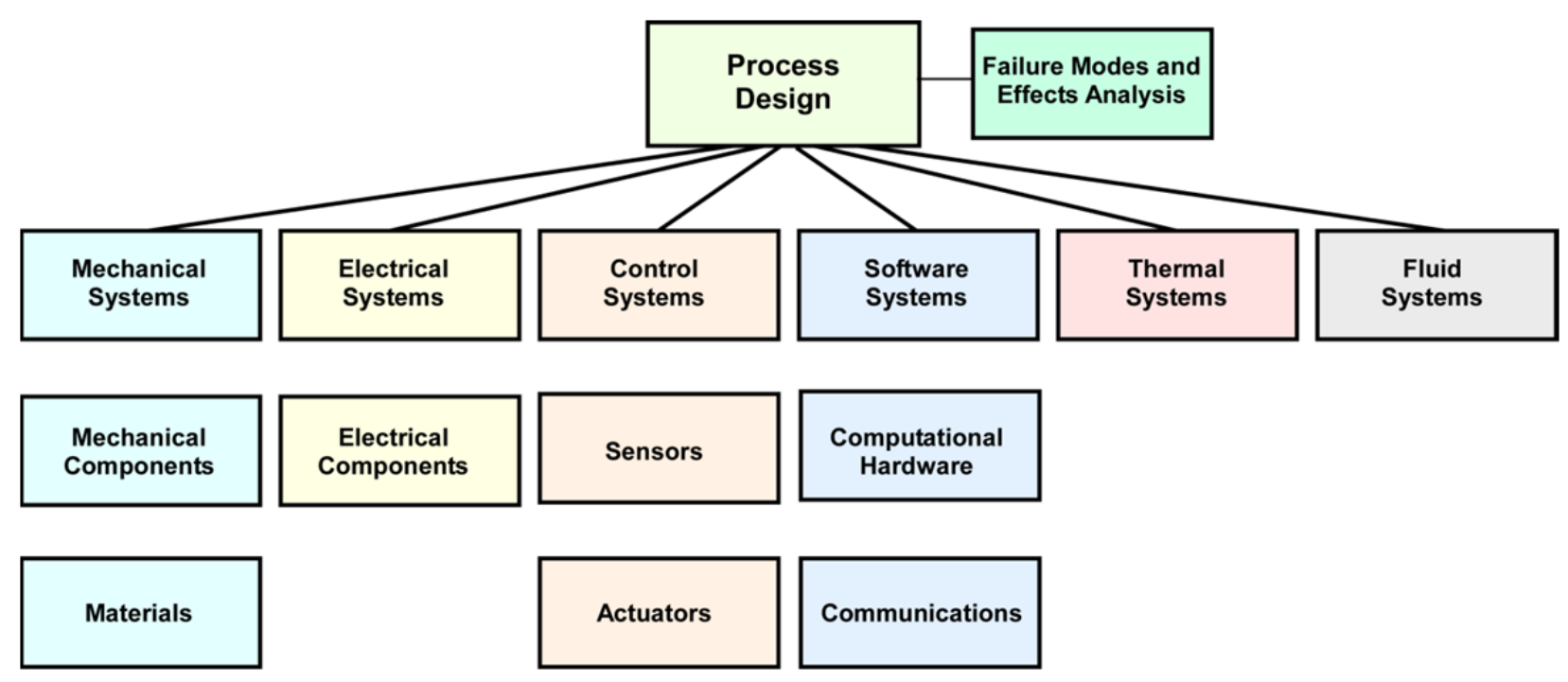

Fig. 1. Systems engineering integrates all involved disciplines into the design process.

Advanced I\&C has the potential to reduce the capital cost and the total life cycle cost required for nuclear power systems. ${ }^{2,3}$ One example of advanced I\&C decreasing capital cost has already become common in the railroad industry where wheel slip detection systems combined with variable frequency ac drive have enabled up to a 50 percent increase in potential thrust per wheel, reducing the number of locomotives necessary to move the same load. ${ }^{4}$ Improved components with deeply embedded I\&C also can make possible performance that is otherwise unattainable. In the defense sector, stable operation of (aerodynamically unstable) delta-wing aircraft is only possible through the continuous corrections made by the flight control system. Limiting the number of replicated instrumentation channels would decrease plant capital cost. Due to current safety requirements, nuclear power instrument channels are deployed with specific minimum channel redundancy. The designer often elects to increase channel redundancy to prevent spurious scrams during testing when one channel is taken out of service. Sufficiently increasing the instrument channel mean-time-between-failure (MTBF), combined with self-testing, would enable plants to only deploy minimally redundant systems as is common in other safety-critical industries. Instrumentation systems can also reduce nuclear plant capital costs by avoiding unnecessary component replacement. Adequate on-line monitoring of component health and process conditions will alert operators of issues such as improper coolant chemistry or component degradation. A dramatic example where improved monitoring would have averted a large capital expenditure is the Davis-Besse reactorhead corrosion incident. ${ }^{5}$ On-line monitoring of either head penetration leakage or the integrity of the reactor vessel head could have prevented the unsafe condition from occurring as well as the costs of the long shutdown and repair.

\subsection{PROJECT PURPOSE}

The practical objective of this work is to tackle a difficult harsh environment problem common to many advanced power reactor designs by developing and demonstrating sensors and controls that operate as embedded parts of a system. The embedding concept allows deployment of reliable, high-performance plant components that otherwise would not be possible. A specific motor-pump combination has been long pursued for harsh environmental applications - a high-temperature, magnetic bearing, canned-rotor pump. This system has been chosen as the I\&C demonstration platform for this project. The project is organized to demonstrate how modern $\mathrm{I} \& \mathrm{C}$ technologies such as fault tolerant computing, environmentally tolerant sensors, on-line diagnostics and prognostics, and integrated digital controls can unite to improve NPP SSC. 
Historically, coolant pumps for liquid metals, molten salts, and helium (especially their seals and bearings) have been a limitation to achieving high plant reliability and are an ongoing maintenance burden. Conceptually, the fundamental mechanisms of any pump system are independent of the fluid being pumped; hence, the technologies developed for any modern high-temperature-tolerant pump system will cross-cut several reactor classes. The helium circulator employed in the Next Generation Nuclear Plant Program, the sodium and liquid salt pumps employed by the Advanced Reactor Concepts program, as well as the Small Modular Reactors program would all benefit from this integrated sensing and controls demonstration project.

A pump for liquid-salt reactors was chosen as the specific pump system for the demonstration platform. The pump conceptual design necessarily includes material selection related to the application such as fluid wetted material compatibility for a liquid-fluoride-salt environment. However, essentially the same materials are anticipated to be compatible with liquid sodium. The technology, in general, would also be applicable to pumping liquid lead if lead-compatible materials can be developed. Both the canned rotor and magnetic bearing aspects of the pump are also applicable to a helium or carbon dioxide compressor. However, the impellor blade configuration would be significantly different for a gas system. As a highefficiency pump, a canned-rotor, magnetic-bearing design would be especially useful for sodium-cooled reactors because the induction pumps currently used have low efficiency (as low as 10 percent) ${ }^{6}$ compared with centrifugal pumps (at over 85 percent). Moreover, induction pumps shut off abruptly (with no coast down) in the event of a loss-of-power accident. Further, vertical shaft pumps where the motor is outside of the liquid have the potential under accident conditions to entrain gas into the pumped fluid and can thus potentially provide a positive reactivity insertion into the core.

The sensing and control technologies that the smart pump will demonstrate are as follows:

(1) Coupled, multi-axis, high-speed position control,

(2) Intrinsic sensing in support of operations and maintenance,

(3) Embedded high-temperature sensors,

(4) Fault-tolerant computing and controls including graceful degradation, and

(5) High-temperature magnetic actuators.

The Advanced High Temperature Reactor (AHTR) is a design concept for a central generating station type fluoride-salt-cooled high-temperature reactor (FHR). The overall goal of the AHTR development program is to demonstrate the technical feasibility of FHRs as low-cost, large-size power producers while maintaining full passive safety. The general characteristics of a FHR are as follows:

(1) use of coated-particle ceramic fuel,

(2) use of low pressure,

(3) pool-type primary system configuration,

(4) delivery of heat at temperatures greater than $600^{\circ} \mathrm{C}$,

(5) strong passive safety features (no requirement for active response to avoid core damage or large offsite release following even severe accidents), and

(6) use of fluoride salt as a primary coolant.

An FHR configuration is illustrated in Fig. 2. The figure shows a nuclear reactor core and remaining power cycle of a plant. There could be multiple loops that comprise the intermediate heat transport system; hence, for a three-loop design, six salt coolant pumps would be required. 


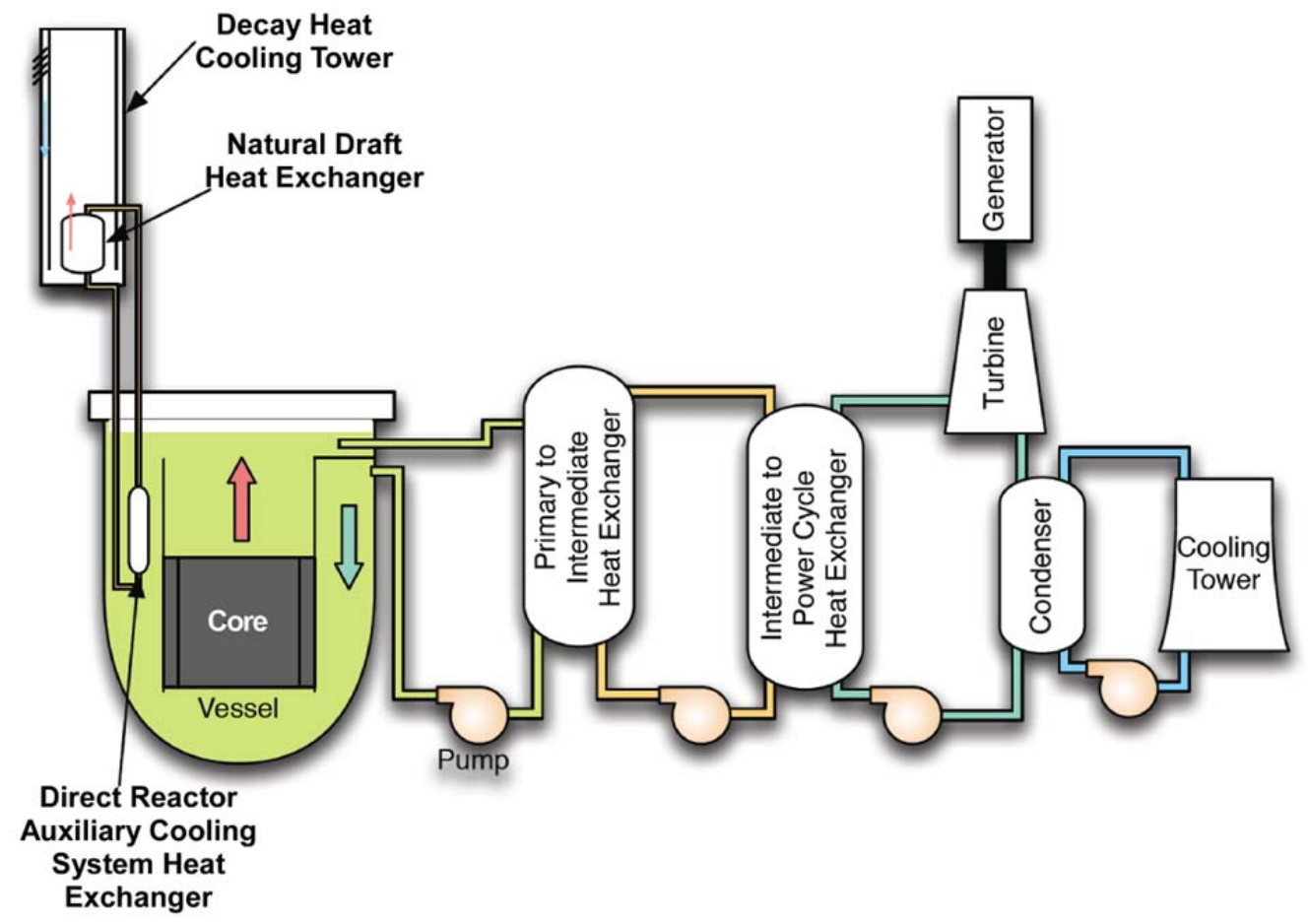

Fig. 2. FHR Design showing various pump locations.

\subsection{BASIC DESIGN CONCEPT}

The design intent of this project is to show that advanced embedded control and sensors can enable highperformance, high-reliability nuclear systems. The physical device being developed is a horizontal shaft, single-stage, centrifugal, high-temperature liquid pump that is compatible with liquid fluoride salts. The pump will feature both a canned rotor and active magnetic bearings. In a canned rotor pump, the rotor of the pump motor is located entirely within the liquid and thin-walled containers separate both the rotor and stator from the fluid. All of the stator windings are isolated from the fluid by the stator can. In a magnetic bearing pump, the rotor is prevented from mechanically contacting the container by active magnetic bearings. A good general overview of magnetic bearings is available in "Magnetic Bearings and Bearingless Drives" by Chiba et al. ${ }^{7}$

The pump motor will employ a switched reluctance-type drive. A switched reluctance motor was chosen due to the high-temperature environment. The desired operating temperature means that the motor design cannot employ permanent magnets. The switched reluctance motor, while being the most simple electromechanical motor design, is the most complex to control and requires high-fidelity position and rotational velocity measurements, which is a challenge in this application. The control of switched reluctance motors is more intimately coupled to the machine design than in other motors. A good overview of the design principles for switched reluctance motors is available in Praveen Vijayraghavan's 2001 dissertation "Design of Switched Reluctance Motors and Development of a Universal Controller for Switched Reluctance and Permanent Magnet Brushless direct current (dc) Motor Drives". ${ }^{8}$ A six-statorpole, four-rotor-pole configuration has been selected as the initial design candidate.

\subsection{ORGANIZATION OF THE REPORT}

- Chapter 2 gives a historical background of advanced control systems and some technological advances made possible by their application. The development of active magnetic bearings and 
advances in motor design are discussed and related high temperature active magnetic bearing work by NASA is presented.

- Chapter 3 outlines the embedded I\&C strategy and approach. The general strategies developed for control system and measurement design will be discussed. In addition, the motor concept will be detailed and the improvements that can be realized in the design phase from the application of FMEA.

- Chapter 4 provides a series of theoretical discussions the mechanical, electrical, and control system design, establishing the methods used to derive the system requirements and conceptual design.

- Chap. 5 details the system requirements; the control system requirements, motor/pump requirements, mechanical design and material considerations, electrical and magnetic performance, sensor considerations, and the mission requirements (performance, reliability, and fault tolerance) are considered collectively to develop specific system design requirements.

- In Chap. 6, the basic conceptual design of the canned rotor pump demonstration platform with embedded I\&C features is described. This conceptual design provides a roadmap toward constructing of the demonstration platform.

- Appendix A contains additional information on the following topics:

- mechanical design (Sect. A.1),

- materials analysis (Sect. A.2),

- position resolver model (Sect. A.3),

- material selection (Sect. A.4), and

- Failure Modes and Effects Analysis (Sect. A.5).

\section{HISTORICAL BACKGROUND}

The objectives for any nuclear power research, development, and demonstration program are to achieve superior cost performance and ensure safe operation. For an AHTR, the role of I\&C in supporting safe operation is less critical than most light-water reactors (LWRs) due to the passive safety characteristics. The remaining safety role is to provide assurance that the passive safety features are not compromised and to provide diverse actuation of mitigation functions. Due to the higher degrees of passive safety provided by AHTRs, the instrumentation can focus on improving cost performance. An AHTR has the potential to decrease capital costs, decrease maintenance costs, decrease insurance costs, increase power production, and increase plant availability. These improvements are made possible in part by deeply embedded sensors and controls in the AHTR.

Progress has been made in several technical areas related to embedding of sensors and controls, control of motors including high-temperature motors and canned rotor motor-pump systems, and sensorless measurement systems. These topics are briefly discussed because of their direct relevance to embedding sensors and controls in a crucial high-temperature component. A short description is also provided regarding the high-temperature liquid-salt test loop constructed at Oak Ridge National Laboratory (ORNL).

\subsection{ADVANCES ENABLED BY EMBEDDED SENSORS AND CONTROLS}

Control technology developed during the Apollo program was a catalyst for safer and more efficient aircraft. In the late 1960s, engineers at the National Aeronautics and Space Administration (NASA) Flight Research Center (now NASA Dryden) proposed replacing bulky hydro-mechanical flight-control systems 
on aircraft with much lighter weight and more reliable analog fly-by-wire technology. On 25 May 1972, the successful testing of the world's first-ever digital fly-by-wire (DFBW) technology on a modified F-8 Crusader jet fighter precipitated an evolution in aircraft design and performance. ${ }^{9}$ DFBW technology was enabled by advances in electronics, computation, algorithms, sensing, and electro-mechanical systems.

For military aircraft, the deployment of DFBW control systems allowed the development of highly maneuverable fighter aircraft and the improvement of their "carefree handling" performance and combat survivability by preventing stalling, spinning, and tolerating actuator hydraulic failures. In the commercial airline market, Airbus introduced full-authority fly-by-wire controls in 1988 with the A320 series, followed by Boeing with their B-777 in 1995. The primary benefits were (1) a reduction of the airframe weight by smaller, lighter aerodynamic control surfaces and (2) increased aircraft safety and reliability. ${ }^{10}$ Commercial airline accident rates have been reduced by a factor of 10 over a 20 year period with computer-assisted control that enables the pilot to safely land an aircraft with various minor or severe failure conditions. ${ }^{10}$ Military applications have developed active control to provide suitable flighthandling qualities for system failures, battle damage, and adverse weather conditions. ${ }^{10}$

Process industries deployed digital distributed control systems (DCSs) starting in the 1970s which provided improved operation versus previous nondigital systems. The DCS digital version of the proportional-integral-derivative (PID) algorithm limited process efficiency due to poor handling of constraints, poor performance with multivariable processes, and poor user interaction. ${ }^{11}$ DCS products began to offer a new control approach described as model predictive control (MPC) in the 1980s, which provided significant improvements in process efficiency. Over the 1960s and 1970s, Shell Oil engineer Charles Cutler worked on ideas to avoid the PID controller operating on multiple constraints, which negatively affected process efficiencies and yields. From this work, a theory of applying real-time process dynamic models to the control became dynamic matrix control (DMC) which later became MPC. Circumstances prevented application of this theory until a strike in 1973. The union strike required a unique need to operate fluid catalytic cracking unit without human supervision. This was not possible with the standard PID controller method. Cutler applied a version of real-time DMC to this process, and it operated with higher process efficiency and yield during nominal operation and failure mode events. ${ }^{12}$

Commercialization of this improved control algorithm was limited prior to the 1980 s because the commercial products were not suitable for implementation of a DMC or MPC. Today, many commercial offerings for MPC exist. The adoption of MPC versus previous approaches results in improved process throughput, reduced energy consumption, reduced emissions, and improved product quality for various chemical, petrochemical, energy, pharmaceutical, mining, metal, and other process industries. ${ }^{10}$

\subsection{MOTOR AND BEARING DEVELOPMENT AND APPLICATION}

Prior to the 1980s, switched reluctance motors experienced almost no commercial application due to high torque ripple, rough running, and high costs associated with the thyristor electronic and control hardware. The period from the late 1960s to the 1980s benefited from several developments to enable key gains in switched reluctance machine performance and cost.

1. Power electronics developed from the bipolar junction transistor (BJT) to the power metal oxidesemiconductor field-effect transistor (MOSFET) and later to the insulated-gate bipolar transistor (IGBT) facilitated forced commutation and pulse-width-modulated (PWM) current regulation at higher frequencies than permitted by thyristor devices.

2. Microprocessors developed capabilities for control that is more complex and switching algorithms to improve regulation of current, speed, and torque.

3. Computer simulation tools advanced to support better simulation and design of the magnetics, electronics, and control algorithms. 
4. Variable speed alternating current (ac) drives and high-performance motion control systems expanded into various industries and applications. ${ }^{13}$

Today the switched reluctance motor is found in computer printers, forklift motor drives, clothes washing machines, vacuum machines, locomotive air-conditioning, and NPP control-rod actuation. ${ }^{13,14}$

Despite many benefits and advantages, magnetic bearings were not common even late in the twentieth century due to several reasons.

1. Low-cost digital processors could not provide sufficient computation speed and functionality.

2. Power electronics were prohibitively expensive.

3. Controls design was very specialized due to inherit lack of stability.

4. Shaft displacement sensors were expensive and required unreasonable space.

5. Strong mechanical and electrical system engineering was needed. ${ }^{15}$

In the twenty-first century, advances such as high-speed analog-to-digital converter electronics, threephase pulse-width-modulated (PWM) digital processing and field programmable gate arrays (FPGAs), low-cost power electronics, and wider experience in controls design methods have enabled application of magnetic bearings to applications such as ultrahigh-speed motor drives, harsh environments, and compact systems with high rotational speeds. ${ }^{15}$

\subsection{HARSH ENVIRONMENT WORK BY NASA}

NASA contracted Aerojet-General to design and develop a power system (SNAP-8) that would convert heat from a nuclear reactor into $35 \mathrm{~kW}$ of useful electrical power to provide a nuclear power system for use in outer space. A high-temperature pumping system $\left(1170^{\circ} \mathrm{F} / 632^{\circ} \mathrm{C}\right)$ was developed to pump the NaK heat exchange fluid. ${ }^{16}$ The system consisted of a canned-motor pump with NaK lubricated bearings. To provide adequate cleanliness for the bearings, a small volume of $\mathrm{NaK}$ fluid was isolated within the motor and bearing cavity, and a separate circulation system was provided including a heat exchanger, oxide particle trap, and a 5-micron filter for removing solid particles. With this system in place, the temperatures of the motor and bearings were controlled within acceptable limits. The stator was sealed by an Inconel ${ }^{\circledR}$ can. The rotor was completely sealed by an Inconel ${ }^{\circledR}$ can to prevent the rotor materials from being attacked by $\mathrm{NaK}$. Motor testing was performed at frequencies of $60,95,220$, and $400 \mathrm{~Hz}$. Based on test results, the eddy-current electrical power loss in the stator can was found to be 158 watts at $1170^{\circ} \mathrm{F}$, comparable to the stator iron loss, whereas the rotor can loss was much smaller, just 20 watts. NaK eddy current losses, however, were calculated to be 351 watts. Several NaK pumps were built and tested for a total of 31,136 hours. The maximum accumulated time on a single unit was 10,362 hours including 786 start cycles. The engineering team concluded, based on the test results, that an endurance goal of 40,000 hours was achievable.

NASA invested considerable effort into the selection and testing of electrical conductors and insulators suitable for advanced space electric power systems over the temperature range up to $1600^{\circ} \mathrm{F}\left(870^{\circ} \mathrm{C}\right)$. Thermophysical, electrical, and mechanical property data were obtained on electrical conductor and electrical insulation materials of interest for applications including ac motors, ac generators, dc solenoids, and transformers. Electrical conductor and insulation materials suited for use as magnetic wire and interlamination insulation were specifically included. Over 1200 specimens were prepared and 3500 tests performed at elevated temperature in air, inert gas, or vacuum were evaluated. ${ }^{17}$ Electrical insulation materials suitable for operational lifetimes of greater than 10,000 hours in the temperature range of 1200 to $1600^{\circ} \mathrm{F}\left(620-870^{\circ} \mathrm{C}\right)$ proved to be very limited. 
NASA also invested considerable effort into the selection and testing of magnetic materials suitable for advanced space electric power systems, over the temperature range up to $1600^{\circ} \mathrm{F}\left(870^{\circ} \mathrm{C}\right)$. For applications that included ac motors, ac generators, dc solenoids, and transformers, the candidate magnetic materials included both Hiperco ${ }^{\circledR} 50$ and Hiperco ${ }^{\circledR} 27$. Magnetic properties were typically measured up to $1400^{\circ} \mathrm{F}\left(760^{\circ} \mathrm{C}\right)$. Interlaminar insulation primarily consisted of either aluminum orthophosphate or mica aluminum orthophosphate bentonite. After a series of winding insulation failures, a more suitable winding technique was devised that proved satisfactory. Windings were sectionalized to reduce voltage requirements for the high-frequency testing up to $3200 \mathrm{~Hz}$. Anadur insulated nickel-clad copper wire was used for the coil windings, with additional Fiberfrax mat insulation added between layers within the windings. ${ }^{18}$ Ceramic tubes were used to bring out all wire through the windings. Additional details and magnetic test data were updated in subsequent reports, ${ }^{19}$ one of which included the use of Anadur insulated nickel-clad silver wire. ${ }^{20}$

Extensive testing of high-temperature electrical components under vacuum conditions was performed at Westinghouse Electric Corporation for NASA. ${ }^{21}$ Tests included two 5000 hour aging tests of a 3-phase stator, a step-down transformer, and two dc solenoids at temperatures of $1100^{\circ} \mathrm{F}\left(593^{\circ} \mathrm{C}\right)$, and at $1300^{\circ} \mathrm{F}$ $\left(704^{\circ} \mathrm{C}\right)$, under vacuum conditions. For the $1300^{\circ} \mathrm{F}$ stator test, the interlaminar insulation for the $0.008 \mathrm{in}$. thick Hiperco ${ }^{\circledR} 27$ laminations consisted of a sapphire-like plasma arc spray coating of 99.995 percent $\mathrm{Al}_{2} \mathrm{O}_{3}$, with 0.000117 in. thickness. The conductor wire was Inconel ${ }^{\circledR}$-clad silver (with rectangular 0.091 $\times 0.144$ in cross section) coated with a 0.0006 in layer of Anadur Owens Corning S-glass fiber overcoated with proprietary oxide loaded silicone wire enamel. The potting compound consisted of Boron Nitride fiber cement. Similar materials were used in the construction of both the $1300^{\circ} \mathrm{F}$ step-down transformer test and the $1300^{\circ} \mathrm{F}$ solenoid. To ensure that hot spot temperatures did not exceed $1300^{\circ} \mathrm{F}$ during testing, temperature distributions were measured using large numbers of Inconel ${ }^{\circledR}$ sheathed thermocouple sensors. The $1300^{\circ} \mathrm{F}$ stator survived testing in better condition that the $1100^{\circ} \mathrm{F}$ stator that utilized lower temperature materials. During the course of the $1300^{\circ} \mathrm{F}$ step-down transformer tests, the primary winding in each transformer shorted due to a reduction in winding support during bakeout, allowing turn-to-turn motion, which led to insulation failure. The $1300^{\circ} \mathrm{F}$ solenoid test also experienced a coil failure, as evidenced by silver migration.

In preparation for the Venus Lander Mission, high-temperature motors capable of long-term operation in an ambient environment of $460^{\circ} \mathrm{C}$ were needed. To ensure performance, most components were designed for long-term use at $540^{\circ} \mathrm{C}$. Requirements included a high-temperature brushless dc motor and resolver, a high-temperature drill, and a high-temperature switched reluctance motor (SRM), all of which were developed and tested by Honeybee Robotics Spacecraft Mechanisms Corp. ${ }^{22}$ As of 2008, an SRM had been operated noncontinuously over a 20 hour period at Venus-like conditions, $460^{\circ} \mathrm{C}$ in a $\mathrm{CO}_{2}$ gas environment. The brushless dc motor was operated (together with the resolver) at temperatures up to $460^{\circ} \mathrm{C}$ at speeds up to $1000 \mathrm{rpm}$. The high-temperature drill consisted of two switched reluctance motors integrated with gears, bearing, and bushings to provide the required drive axis capability. Both motors were driven by a custom Honeybee controller using a sensorless control algorithm. The drill performed well during 20 hours of testing under Venus-like conditions $\left(460^{\circ} \mathrm{C}\right.$ in $\mathrm{CO}_{2}$ gas), including successfully drilling into chalk to a depth of 6 in.

In addition, a high-temperature magnetic bearing was operated at $1000^{\circ} \mathrm{F}\left(540^{\circ} \mathrm{C}\right)$. The design and fabrication, including the development of high temperature wire, and a coil fabrication process, were reported by Montague et al. ${ }^{23}$ Test data acquired during and after 11 hours of operation at $1000^{\circ} \mathrm{F}$, and multiple (six) thermal cycles, was described by Provenza et al. ${ }^{24} \mathrm{~A}$ high-temperature switched reluctance motor capable of speeds up to $8,000 \mathrm{rpm}$ was operated at $1000^{\circ} \mathrm{F}\left(540^{\circ} \mathrm{C}\right)$, for $>27.5$ hours, including 14 thermal cycles. ${ }^{25}$ 


\subsection{PRIOR SALT COOLANT PUMPS}

Pumps for coolant or nuclear fuel circuits have been under development for fluoride salt reactor applications since the early 1950s. Salt pumps have also been used in a number of material processing industries, including aluminum production, and are used with nitrate salts in solar thermal energy systems. $^{26}$

High-temperature pumps have been developed for both circulating salt and liquid-metal systems, with many common features between the two. Some small pumps, such as those used for in-reactor test loops, have been compact units driven by gas or hydraulic fluid. Simple salt transfers can be made by pressurizing a vessel with a cover gas, forcing salt out through a dip tube. The most common type of pump used has been the overhung, vertical shaft centrifugal pump. These are usually located with the pump bowl at an elevation with a gas-liquid interface. The motor is above the salt surface; the pump bowl fills with salt. They are further categorized by the location of the shaft bearings. The short-shaft variant has a lower bearing near the pump impeller, immersed in and lubricated by the salt. This type of pump was more successful in liquid-metal applications. The long-shaft variant, more common in salt applications, has all bearings located well above the salt surface. ${ }^{27}$

During the development of the Molten Salt Breeder Reactor (MSBR), a number of forced circulation loops were constructed. Several standardized pumps were developed and fabricated by ORNL, almost all of the long-shaft variant. The LFB pump ${ }^{28}$ was used on several loops. It had two grease-lubricated, sealed bearings with an oil-lubricated face seal below the lower bearing. The motor was located next to the pump, connected by belts that were selected for the desired operating speed. In the Alpha pump, used in some of the final loops constructed prior to cancellation of the MSBR program, the belt drive was replaced with a variable frequency motor driven by large motor-generator sets. ${ }^{29}$

The fuel salt pump used in the Molten Salt Reactor Experiment (MSRE) was the result of an extensive development effort. ${ }^{30,31}$ It was an overhung centrifugal pump of the long-shaft variety, designed for remote installation, maintenance, and removal. It delivered 1,200 gpm at a discharge head of $49 \mathrm{ft}$, operating at $1160 \mathrm{rpm}$. A sketch of this pump is shown as Fig. 3, and a photograph is provided as Fig. 4. The MSRE fuel pump was located at the high point in the fuel salt circulating system and served a number of additional key functions. Salt entered the bottom of the pump bowl and was discharged horizontally. The salt level in the circuit was adjusted in the pump bowl; the top of the bowl contained helium cover gas, and an adjacent overflow tank prevented complete immersion of the bowl in certain operational transients. A shield plug immediately above the bowl reduced the radiation exposure for components above the bowl. There were two sets of ball bearings, lubricated and cooled by an external oil circuit. The oil system was also used to cool the radiation shield. There were graphite/steel plate seals above and below the bearings; oil flow paths are seen in Fig. 3. A separate drain to collect oil seepage below the lower oil seal is also shown. The water-cooled, $75 \mathrm{hp}$ motor is located above the bearings. The entire pump assembly was mounted in a complex support structure designed to allow movement in the horizontal and vertical planes as the system temperature rose, while maintaining the axial orientation of the pump. Extensions to the mounting bolts, allowing remote connection and disconnection, are seen in Fig. 4. 


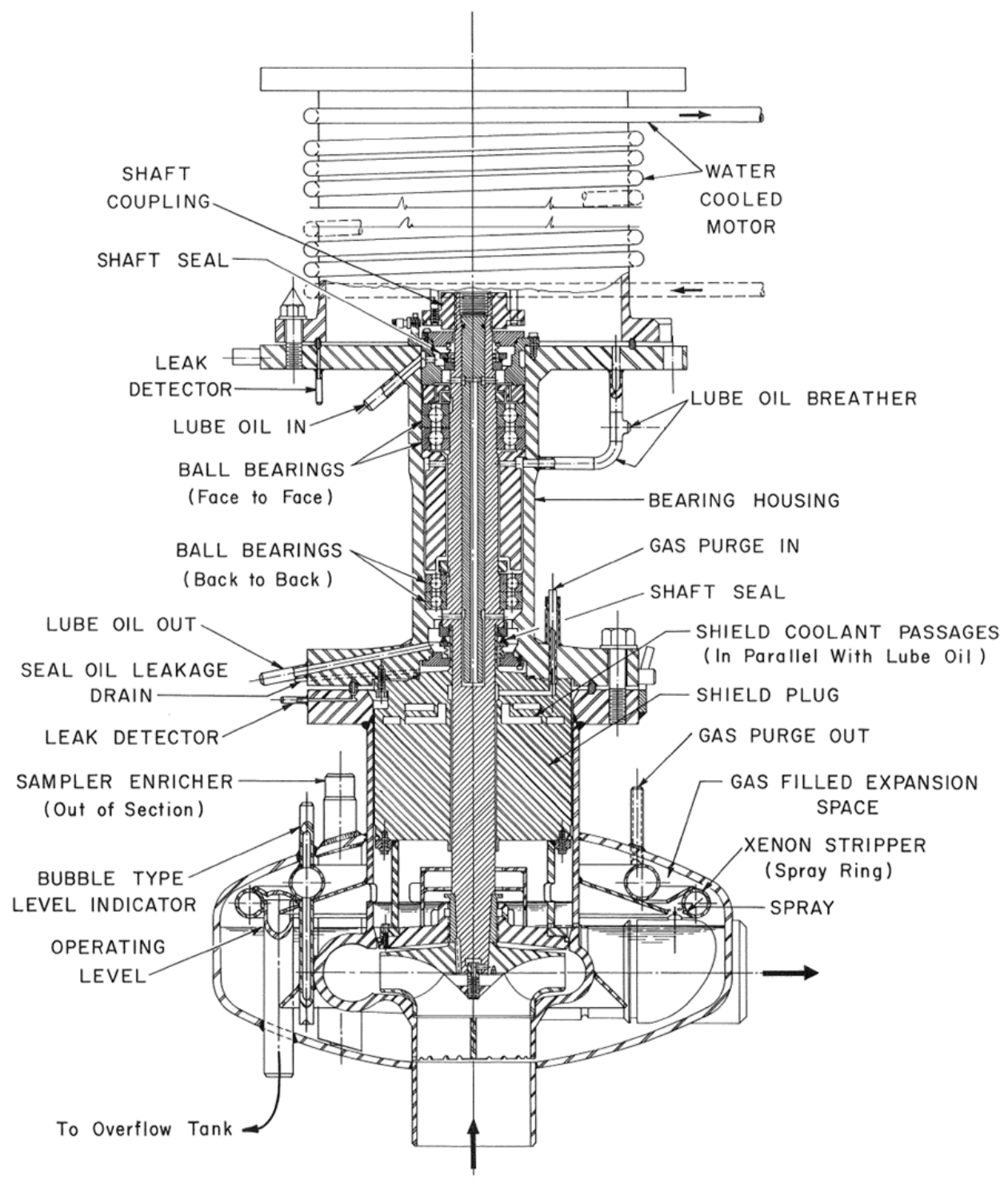

Fig. 3. Sketch of the MSRE fuel salt pump. 


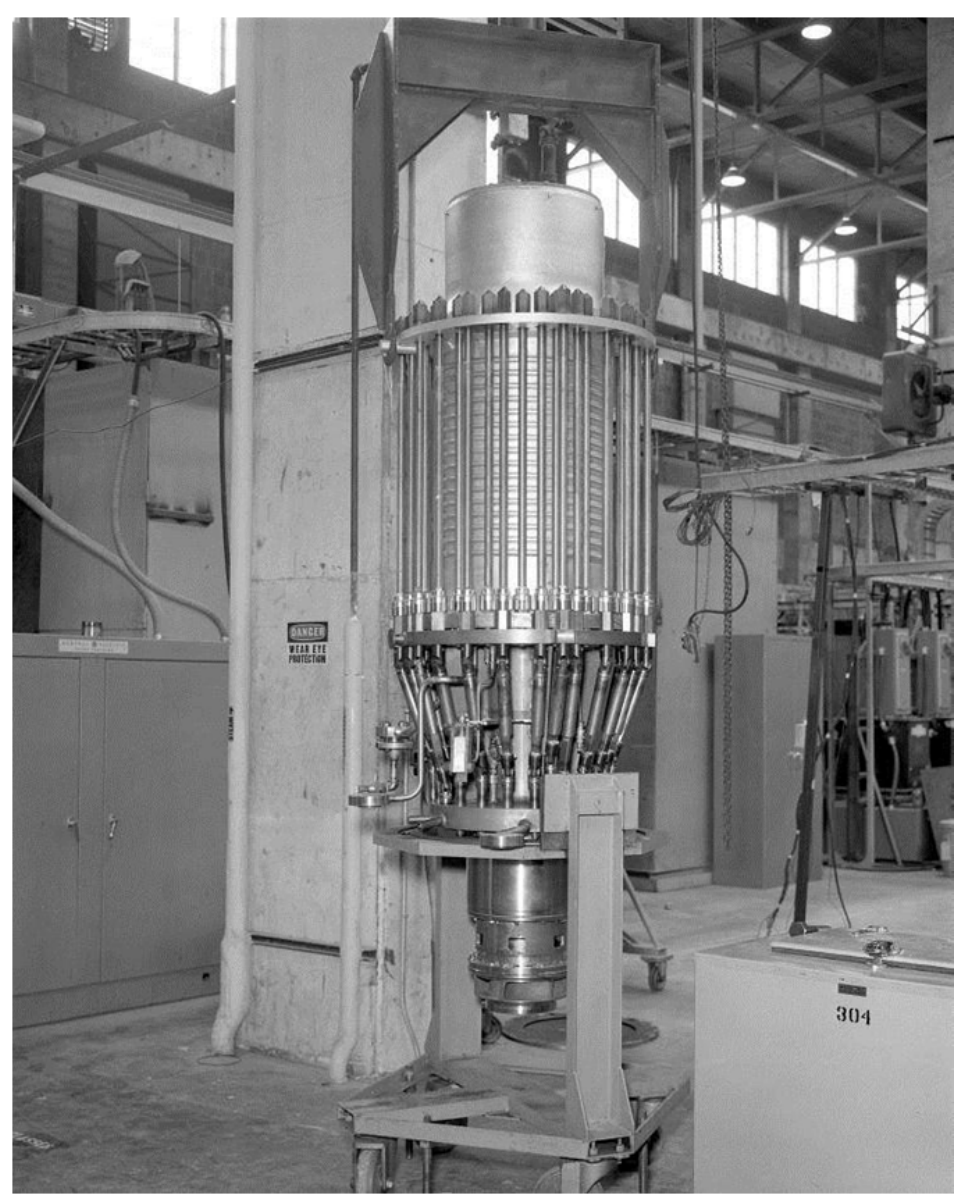

Fig. 4. MSRE fuel salt pump at the pump test facility.

The fuel pump bowl was used for a number of other operations. A tube into the pump bowl was used to collect salt samples for analysis; this facility was also used to add concentrated uranium fuel to maintain criticality during operation, and to add beryllium or nickel to maintain salt chemistry. A bypass from the salt discharge line was directed to a spray ring in the bowl gas space. A series of perforations in this ring allowed salt to be sprayed into the cover gas, so that xenon and other volatile fission gases could be carried off in a sweep of the helium cover gas.

The pump generally performed well during the 5 years of MSRE operations. The most notable operational difficulty associated with the fuel pump was frequent plugging of the helium off-gas lines - likely the result of oil leakage that travelled down the pump shaft into the salt. There it was converted into a tar-like substance that built up on valves and other components in the off-gas system. There was also some evidence of foam and bubbles in the pump discharge, with foaming enhanced by the presence of the spray ring in the pump bowl. ${ }^{32}$ This appeared to alter the void fraction in the core, leading to minor power oscillations.

Because of the heat and radiation near the pump, there was no instrumentation on the pump itself. Motor data were limited to the power drawn from the pump electrical power feed. Some indication of performance could be obtained from the lubricating and cooling oil backpressure and temperature rise. The sample port in the pump bowl presented another opportunity to assess pump performance; information on foaming characteristics was obtained by lowering metal strips into the sample port and observing the salt coating pattern on the strips. 
Most current industrial applications of salt pumps involve batch transfers from open salt baths, and use similar overhung salt pump technology. Most of these pumps are not designed for continuous, long-term operation, and a tightly sealed system is not required. Often, nitrate or chloride salts are used, at lower operating temperatures. A few manufacturers offer canned rotor or other sealed pump systems but may require custom designs or further development to incorporate high-temperature operation using materials compatible with fluoride salts.

Recent loops constructed at ORNL, the University of Wisconsin, and other locations continue to be based on the overhung, long-shaft centrifugal pump technology. An example is the fluoride salt loop constructed at ORNL to demonstrate cooling of graphite pebbles using a eutectic LiF-NaF-KF salt mixture (FLiNaK). The pump itself is a simple sump-type pump, immersed in a slightly pressurized sump tank with an argon cover gas. The pump assembly itself is shown in Fig. 5. A close-tolerance gas-buffered seal is located just above the flange, and there are two sealed, grease-lubricated ball bearing sets above the seal. The pump supplies $4.5 \mathrm{~kg} / \mathrm{s}$ of salt at a $0.125 \mathrm{MPa}$ head. A $5 \mathrm{hp}$ variable frequency motor is mounted to the loop frame above the sump tank, coupled to the pump shaft by a flexible drive shaft.

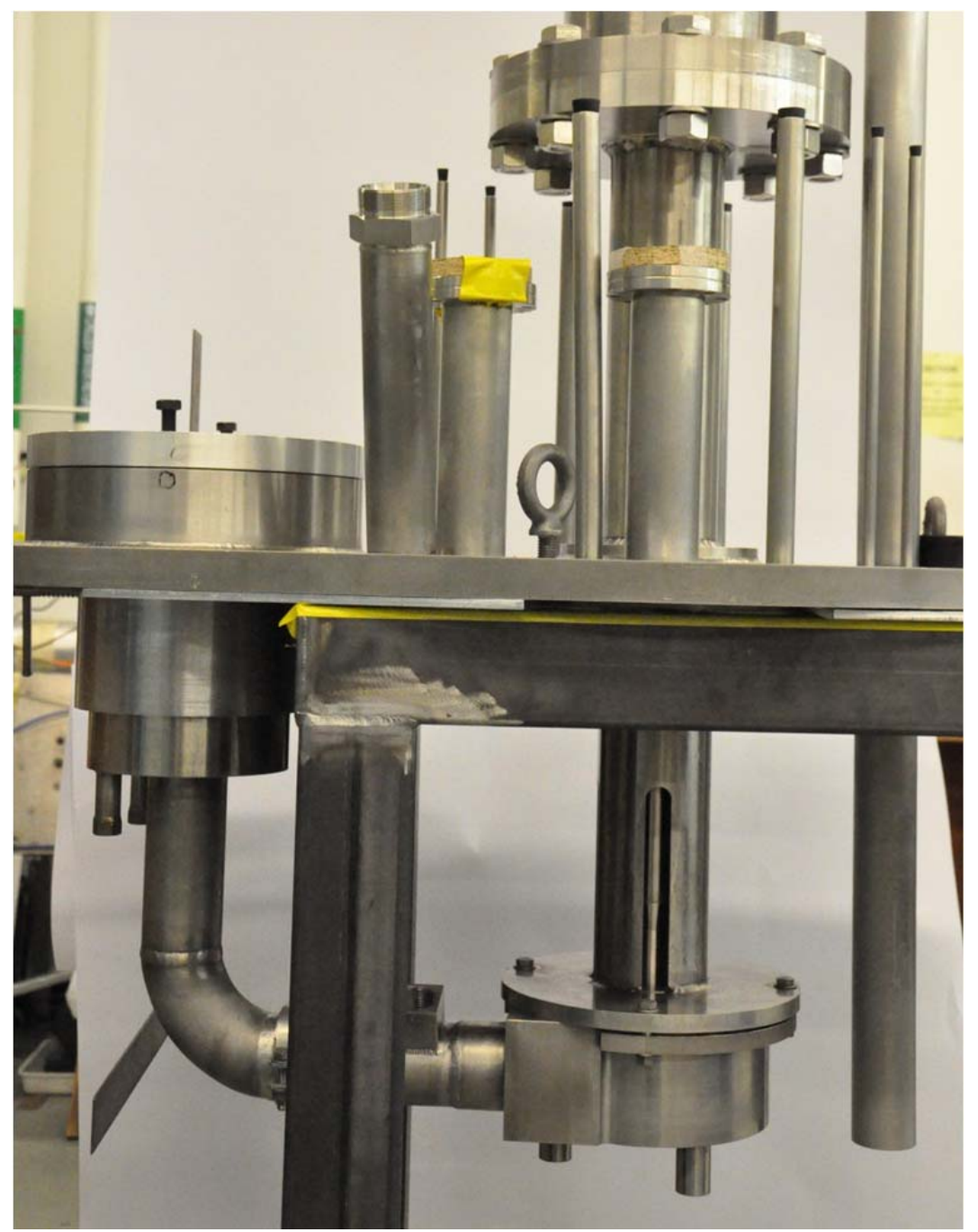

Fig. 5. Sump-style salt pump for inductive-heating loop.

The primary source of data for this pump comes from the solid-state variable-frequency drive unit that provides power to the motor. The primary control point is pump speed. Further operating characteristics 
can be inferred by monitoring the current draw. The pump output is measured by a pressure transmitter that is coupled to the pump outlet leg, and a flow meter on the salt lines outside the sump. A description of the inductive heating loop follows.

\subsection{SALT LOOP DEMONSTRATION AT ORNL}

A small forced-convection liquid-fluoride-salt loop has been constructed at ORNL to examine the heat transfer behavior of FLiNaK salt in a heated pebble bed. Objectives of the salt-loop experiment include reestablishing infrastructure needed for fluoride-salt loop testing, developing an inductive heating technique for performing heat transfer experiments, measuring heat transfer characteristics in a liquidfluoride-salt-cooled pebble bed, and demonstrating the use of silicon carbide (SiC) as a structural component for salt systems. The salt loop consists of an Inconel ${ }^{\circledR} 600$ piping system, a sump-type pump, a $\mathrm{SiC}$ test section, and an air-cooled heat exchanger, as well as auxiliary systems needed to pre-heat the loop, transport salt into and out of the loop, and maintain an inert cover gas over the salt. A $30 \mathrm{kHz}$ inductive heating system provides up to $200 \mathrm{~kW}$ of power to a $15 \mathrm{~cm}$ diameter $\mathrm{SiC}$ test section containing a packed bed of $3 \mathrm{~cm}$ graphite spheres. A SiC-to-Inconel ${ }^{\circledR} 600$ joint will use a conventional nickel/grafoil spiral wound gasket sandwiched between $\mathrm{SiC}$ and Inconel ${ }^{\circledR}$ flanges. The loop system can provide up to $4.5 \mathrm{~kg} / \mathrm{s}$ of salt flow at a head of $0.125 \mathrm{MPa}$ and operate at a pressure just above atmospheric. Pebble Reynolds numbers of up to 2600 are possible with this configuration. A sump system is provided to drain and store the salt when not in use. Instrumentation on the loop includes pressure, temperature, and flow measurements, while the test section will be instrumented to provide pebble and FLiNaK temperatures. ${ }^{33}$

At a future date, the intention is to replace the sump-type pump currently in the salt loop with a prototype pump fabricated according to the conceptual design of this report. The loop provides an excellent test bed and demonstration of an embedded canned rotor motor-pump system for harsh reactor environments.

\section{STRATEGY AND APPROACH TO PROBLEM}

At a high level, the overall objective of this project is to develop and demonstrate improved reliability, performance, and practicality of embedding sensors and controls in reactor systems and components that are important for plant operability. These systems and components are determined by establishing functionality of the system and setting the operating environment. The strategies that determine the pathways to achieving the objective are discussed in this section.

\subsection{CONTROL STRATEGY}

A discussion of systems control starts at a high level and establishes the scope of control required. Figure 6 illustrates six control properties that contribute to realizing embedded resilient control.

Traditional feedback control is represented by the far left box, in which natural (expected) changes in the controlled system are compensated for - noise, changing process parameters, and limited exogenous influences. Enhanced reliability and continued performance despite more severe degradation require additional capabilities as expressed by the supplementary properties shown in the figure. Some of the properties shown such as tolerance to human error and cyber security are not directly treated in this development project. Ultimately, a commercialized product intended for use in a reactor environment will be designed for these contingencies as well. 


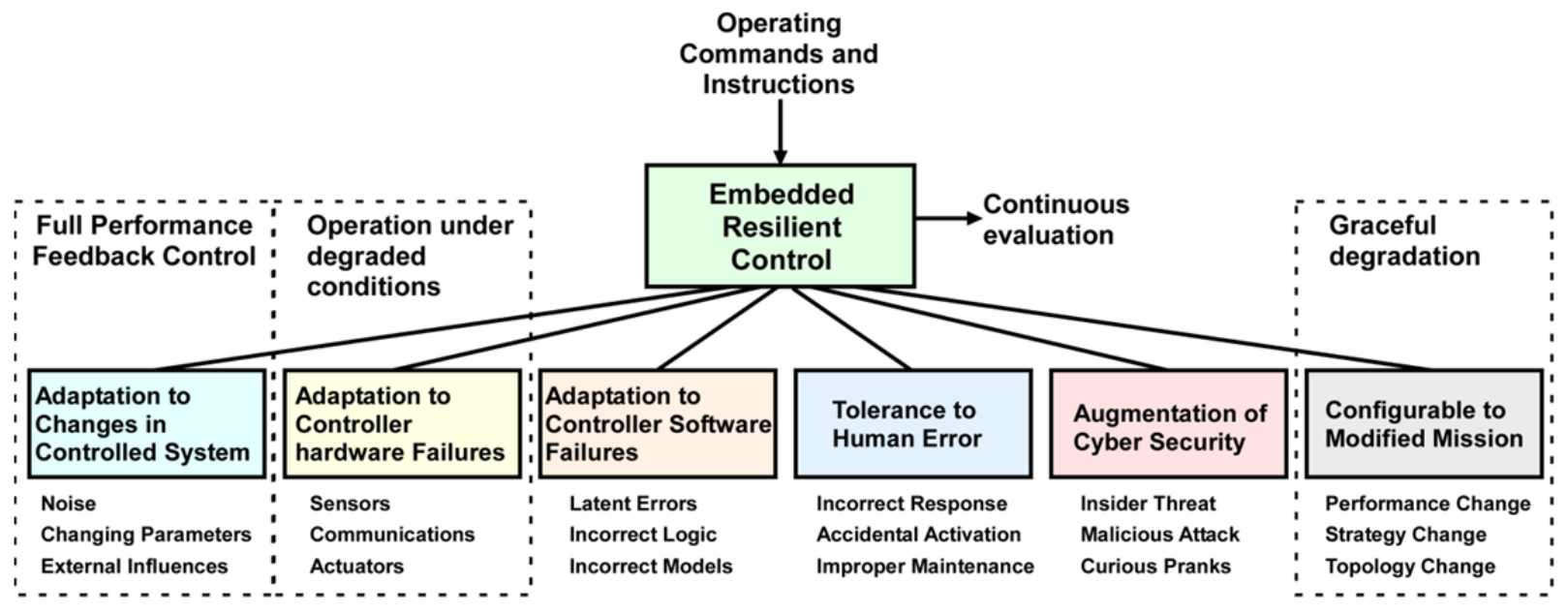

Fig. 6. Graphical representation of scope of control actions.

Referring to the concepts in Fig. 6, control strategies can be applied at multiple levels to achieve the high performance and reliability standard of an embedded approach:

(1) meet the full-performance standard of the system under all normal operating modes (e.g., start-up, running at a select speed, ramping speed, and shutdown);

(2) enable continued operation of the system under degraded conditions including out-of-tolerance components, failed or failing components, and human error; and

(3) degrade gracefully to a safe state for conditions in which it is impossible to continue operation such as for loss of power or the total failure of a major component or subsystem.

In addition to these control strategies, the control system should evaluate immediate and time-evolving conditions to diagnose problems, predict degradation, inform system operators, and interact with maintenance functions. Specific control concepts must be implemented to facilitate the strategies of continued operation under degraded conditions and graceful degradation under major failure.

A simplified block diagram of the motor control system is given in Fig. 7. The diagram shows the measured values coming from sensors or stator windings and output currents and signals going to various stator windings. In addition, command signals and status parameters are communicated to upper levels of the control hierarchy. The internal functions of the control system can be summarized as the blocks indicate - input electronics [e.g., input protection, analog-to-digital (A/D) conversion], signal processing (e.g., filtering, estimation, and model prediction), control calculations (for magnetic suspension, main rotational drive, and temperature control), diagnostics/prognostics, high-frequency current drive, and external communications. 


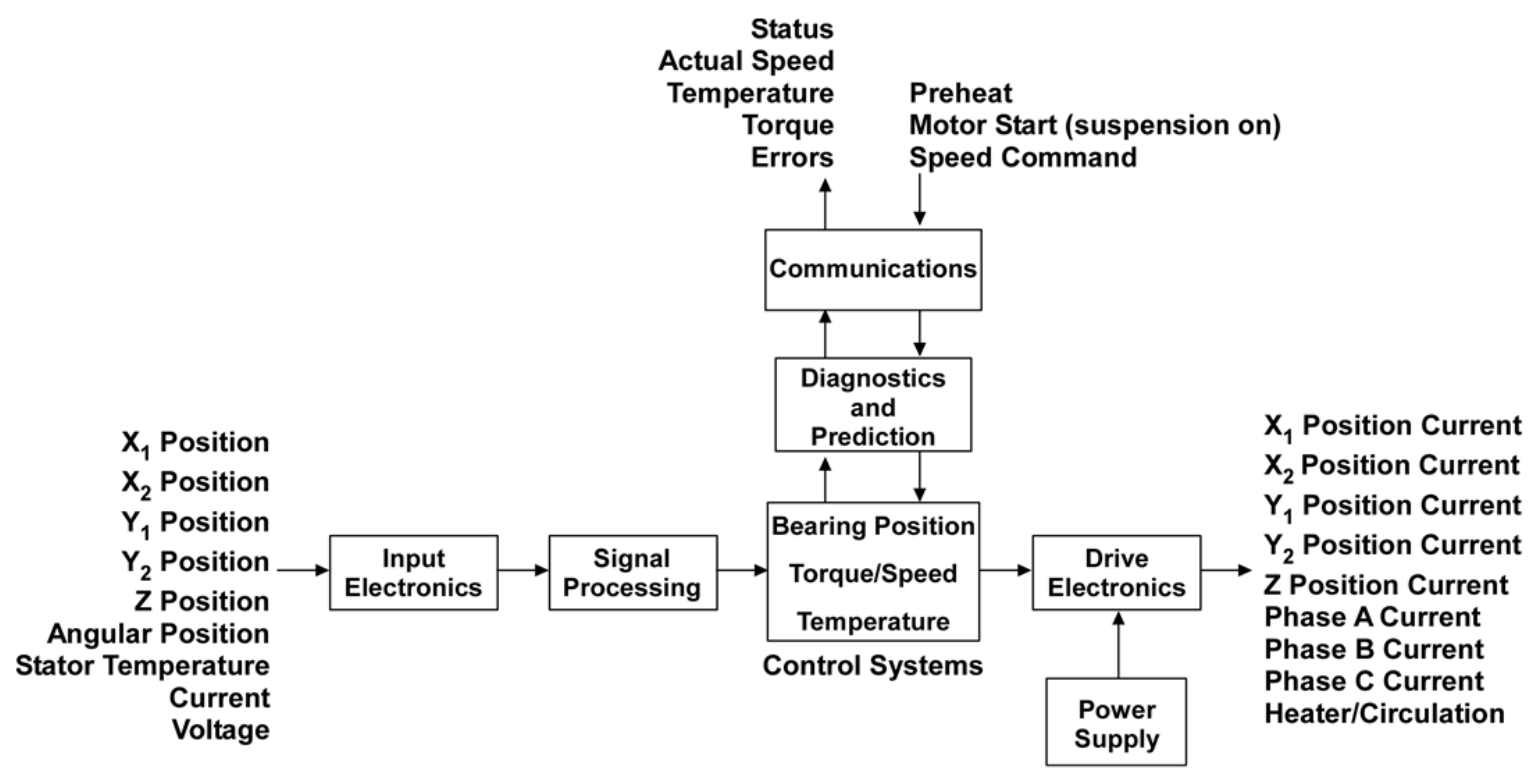

Fig. 7. Simplified system control electronics block diagram.

\subsubsection{Operating Modes}

1. In the startup region, the system must be heated up to be compatible with the desired salt operation temperature $\left(\sim 700^{\circ} \mathrm{C}\right)$ before pumping of the molten salt can occur.

2. During normal steady-state pumping operation, the salt material will be used to remove excess heat from the pump system. If the pump is sufficiently large, external forced cooling may be required.

3. During shutdown, the system will be drained of salt prior to removal of heat by passive or active means.

The cooling salt material provides additional challenges for non-steady-state operating regions. Even the normal operating modes for this demonstration platform present a challenging environment for sensing and control. During startup and shutdown operations the initial rotational position needs to be determined and accurate position and rotational velocity measurements are needed for proper motor control.

Developing sensors that will operate with sufficient resolution and low noise is extremely challenging at low rotational velocities. This large uncertainty and noise introduced during startup need to be accounted for in the control algorithm design. The large temperature change during startup and shutdown causes large changes in the material properties which affect the system dynamics. These parameter variations must be incorporated into the control system. In the following sections we will discuss the normal and degraded operation of the control system.

\subsubsection{Normal Full Performance}

Startup sequence for the canned motor consists of several functions.

- Power inverter dc link filter capacitor pre-charge

- Sequencing of main power contactor and pre-charge contactor

- Regulated logic power supply to control electronics, sensors, and gate drivers

- Sensing home position of rotor and present position for proper stator current injection

- Ramping of stator currents with rotor position to meet specific spin-up time of the pump

- Implement proper controller dynamics to ensure speed tracking during ramp, minimal speed overshoot, and steady state stability 
- Reverse sequencing during shutdown, especially if emergency shutdown

A similar start-up sequence applies to the active magnetic bearings and the motor rotor and pump impeller. The active magnetic bearings require control action in their power electronics to ensure soft liftoff from the touchdown bearings and a smooth transition to the controlled position. Accurate knowledge of rotor position and system temperature is required to accomplish this in a safe and repeatable manner.

Shutdown sequencing may differ from startup sequencing due to inertia of the pump and need to ensure no hydraulic "hammer" in the pumped fluid. The shutdown speed-control ramp may therefore be at a different rate from during start-up. The SRM power inverter must also be controlled during shutdown so that it enters braking mode (generating) in order to absorb kinetic energy of the pump and fluid motion. For the active magnetic bearings, the power inverter controlling their respective stators must execute a soft-landing feature to prevent damage to the touchdown bearings. An impact of the rotor or pump impeller could cause significant damage to the touchdown bearings, rotor, and protective can.

\subsubsection{Operation under Degraded Conditions}

One aspect of the control system will deal with operation under degraded conditions. To do this, prediction functions and detection functions are needed, along with control algorithms designed to be robust to the property changes caused by the degradation. There are numerous potential degradations of the electric and magnetic components in the system. Protection is needed on the rotor to ensure the potentially corrosive fluid does not contaminate the core lamination materials or rotor shaft, but especially the rotor-lamination oxide coating. Loss of magnetic protection would mean an exorbitant increase in rotor loss due to creation of macro eddy currents throughout the bulk of the rotor core and consequent performance loss. Similarly, for the stator, only the pump casing should provide sufficient containment of the pumped fluid to prevent this. However, any deterioration of the stator-coil conductor insulation (a ceramic coating) would lead to fractures and micro-cracks that would form Paschen discharge regions, further loss of electrical insulation, and potential turn-to-turn shorting or worse, a full coil short. The power inverter itself is maintained well outside the pump motor high temperature environment and therefore can be maintained at reasonable operating temperature. Liquid cooled heat sinks and forced-aircooled heat sinks can ensure power-transistor junction temperatures remain below $150^{\circ} \mathrm{C}$ at maximum. In either case, liquid or air cooling, the power inverter will experience from $80^{\circ} \mathrm{C}$ to $100^{\circ} \mathrm{C}$ temperature excursions during initial start-up, but then on a very continuous operating temperature unlike traction inverters in electric vehicles that experience many thousands of temperature cycles and millions of power cycles.

The development of prediction functions, in effect, a state-of-health (SOH) monitoring routine, will preempt failures. There are many candidates for loss-of-performance prediction based on parameter tracking and other estimation techniques. Off-line methods such as Fourier and Wavelet Transform methods offer the ability to sense very subtle performance degradations early and from these to identify in a signature analysis sense, where a failure is likely to occur.

The SRM controller can accommodate parameter degradation within limits and does so provided its control algorithms are designed to do this. The SRM driven fluid pump will operate continuously in a $700^{\circ} \mathrm{C}$ environment. Long-term exposure to high temperature, even for a system designed to do just that, will experience parameter deterioration such as lowering of mild steel permeability and insulation properties. Sudden changes to the system properties are possible too. In real time, detection strategies coupled with robust control techniques will maintain operation under the degraded properties. 


\subsubsection{Continuous Evaluation}

The pump motor controller and active magnetic bearings must be continuously monitored and parameter conditions logged. There are a number of strategies that can yield information about the internal state of the system. For example, with an SRM its phases are sequentially energized leaving an out-going phase during the interval when an in-coming phase is being energized. In a three-phase system, one phase is inert at any given moment and from it parameter evaluations may be executed. By injecting a small known current, a voltage measurement across the phase winding will reveal any deviation from nominal resistance. This resistance measurement in turn can be continuously compared to its estimated value (model) for the present operating temperature. The inductance of stator windings on the canned motor can be obtained from flux linkage (voltage) measurements made as a function of rotor position also on the inert phase. For the active magnetic bearings, continuous monitoring of parameter shift and degradation will be more difficult, but possible, by tracking current, voltage, and temperature measurements.

\subsection{MEASUREMENT STRATEGY}

Several sensors types are used in commercially available motors including those based on capacitive, optical, and electromagnetic transduction methods. Typical capacitive and optically based sensors are not feasible for our design because of the desire is to minimize the number of penetrations across the isolation barrier between rotor and stator and because of the harsh environment (e.g., high temperatures and corrosive salt). Ultrasonic sensors, though not typically used in motor application, are also not feasible because temperatures are above the Curie point for most piezoelectric materials. The transduction strategy for sensors in this motor system is therefore to use or develop magnetic-based devices.

A cross-section illustration of a canned-style motor with rotor and stator components is shown in Fig. 8. During normal operations, the objective of the control system is to drive the rotor shaft at its programmed speed while maintaining the shaft at the centerline of the motor drive axis with the impeller remaining axially centered in the pump casing. To achieve this objective, the control system must be capable of providing a controlled amount of torque to the rotor shaft as well as shaft positioning forces along both axes perpendicular to the shaft. Position control of the shaft needs to be provided at two axial locations to maintain shaft alignment. Additionally, positional control needs to be provided along the shaft centerline in the axial direction.

To provide the necessary shaft torque, the control system needs to know the time history of the shaft rotation angle relative to the stator poles. The time history of the shaft rotation enables determination of the rotational velocity and acceleration. The angle of the shaft relative to the motor stator poles enables determination of which coils to energize to provide rotational force to the shaft. In order to maintain the motor shaft at the centerline of the axis of each motor, the control system needs to know the time history of the magnitude and direction of the shaft offset from the centerline of each drive motor. The axial positioning magnetic bearings can be set up to be inherently stable by having the desired shaft position located between an opposing set of always-energized bearings. However, to minimize the bearing energy expenditure, the time history of the shaft axial position needs to be known to enable only energizing the axial bearing coils to correct shaft misalignment. 


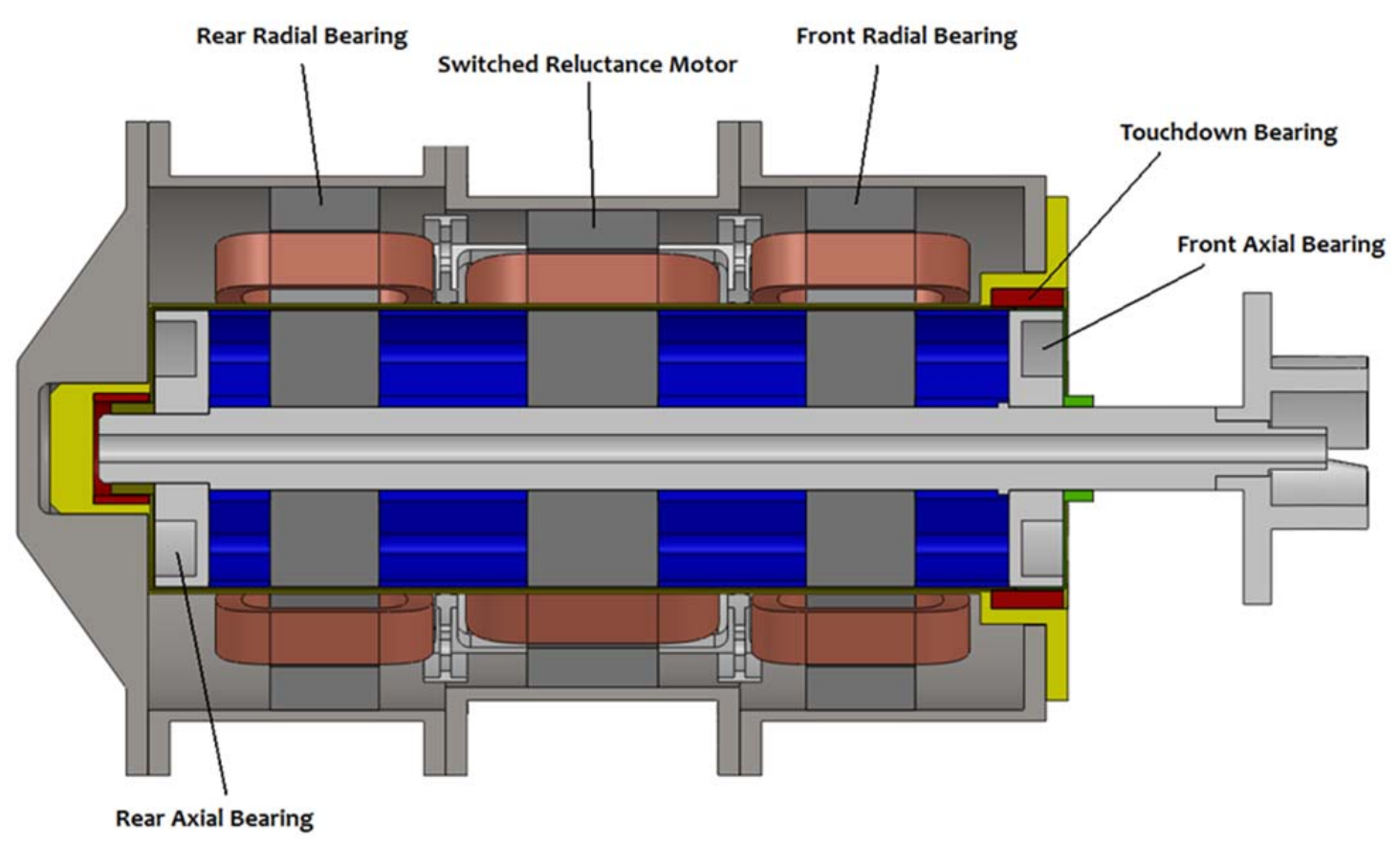

Fig. 8. Reference illustration of canned rotor motor with pump impeller.

A list of the measurements needed for control is summarized in Table 2. Similarly, output signals are summarized in Table 3.

Table 2. Needed measurement signals for specific motor system component

\begin{tabular}{lccccc}
\hline \multirow{2}{*}{ Measurement } & \multicolumn{5}{c}{ Component } \\
\cline { 2 - 6 } & Thrust 1 & Thrust 2 & Bearing 1 & Bearing 2 & Torque \\
\hline $\mathrm{X}_{1}$ Position & $\mathrm{X}$ & $\mathrm{X}$ & $\mathrm{X}$ & $\mathrm{X}$ & \\
$\mathrm{X}_{2}$ Position & $\mathrm{X}$ & $\mathrm{X}$ & $\mathrm{X}$ & $\mathrm{X}$ & \\
Y $_{1}$ Position & $\mathrm{X}$ & $\mathrm{X}$ & $\mathrm{X}$ & $\mathrm{X}$ & \\
Y $_{2}$ Position & $\mathrm{X}$ & $\mathrm{X}$ & $\mathrm{X}$ & $\mathrm{X}$ & \\
Z Position & $\mathrm{X}$ & $\mathrm{X}$ & & & \\
Angular Position & & & & & $\mathrm{X}$ \\
Stator Temperature & $\mathrm{X}$ & & $\mathrm{X}$ & $\mathrm{X}$ & $\mathrm{X}$ \\
Current & $\mathrm{X}$ & & $\mathrm{X}$ & $\mathrm{X}$ & $\mathrm{X}$ \\
Voltage & $\mathrm{X}$ & & $\mathrm{X}$ & $\mathrm{X}$ & $\mathrm{X}$ \\
\hline
\end{tabular}


Table 3. Outputs to motor system components

\begin{tabular}{lcccccc}
\hline \multicolumn{1}{c}{ Drive Signal } & \multicolumn{5}{c}{ Component } \\
\cline { 2 - 7 } & Thrust 1 & Thrust 2 & Bearing 1 & Bearing 2 & Torque & Heating/Cooling \\
\hline $\mathrm{X}_{1}$ Position Current & & & $\mathrm{X}$ & & & \\
$\mathrm{X}_{2}$ Position Current & & & & $\mathrm{X}$ & & \\
$\mathrm{Y}_{1}$ Position Current & & & $\mathrm{X}$ & & & \\
$\mathrm{Y}_{2}$ Position Current & & & & $\mathrm{X}$ & & \\
$\mathrm{Z}$ Position Current & $\mathrm{X}$ & $\mathrm{X}$ & & & $\mathrm{X}$ & \\
Phase A Current & & & & & $\mathrm{X}$ & \\
Phase B Current & & & & & $\mathrm{X}$ & $\mathrm{X}$ \\
Phase C Current & & & & & & \\
Heater/Circulation & & & & & & \\
\hline
\end{tabular}

There are two approaches to implementing the several measurements suggested: (1) independent sensors and (2) sensorless. Our strategy is to employ both approaches to provide redundancy and self-checking ability. The Failure Modes and Effects Analysis (FMEA) results suggest that redundancy is needed for the position measurement. Independent sensors are realized by measurements taken by transduction separate from the current-driven windings for magnetic suspension or the main rotation drive. Later in the report, a rotation and translational resolver adaptable to high-temperature operation is described that accomplishes this independent position measurement function. Sensorless position estimation is often carried out with flux linkage estimation from on-cycle operation, and it is possible to monitor voltages on stator windings during the off cycle.

To accomplish sensorless position measurements requires voltage sensing that can withstand the normal rated voltage (200-400 V), and for off-cycle monitoring techniques, voltage sensing may need to be accurate to the millivolt level. There are also coupling effects between phases that should not be ignored, and electromagnetic interference introduces significant error into voltage, current, and temperature measurement signals. An observer model that includes nonlinear phenomenon would be operated in real time to estimate position. This is a difficult task, and with consideration of other aspects mentioned in Section 4.1, a sensorless approach will likely have higher inaccuracies than a dedicated independent sensor. Nevertheless, the sensorless measurement may have sufficient validity to provide information to the control system under non-transient conditions. Additionally, sensorless angular position measurement is also possible using a "tooth counting" technique, especially with a large inertia system for which an initial position is known.

Implementation of a separate resolver sensor avoids many issues associated with using the motor voltage, current, and resistance as a stand-alone mechanism for position estimation. Additionally, $\mathrm{x}$ and $\mathrm{y}$ axial position feedback can be obtained with a resolver. Although a separate sensor for angular position, $\mathrm{x}$ and $\mathrm{y}$ will be more reliable and accurate, the redundancy and diversity gained can lower risks. The rotor of the magnetic bearing can perhaps be shaped (either with resolver-like radial variant or small teeth for "tooth counting") to provide angular position feedback, and because it is mostly dc, it may be possible to obtain adequate $\mathrm{x}$ and $\mathrm{y}$ locations through inductance profile versus gap mapping. However, using a radial variant on the rotor of the magnetic bearing for identification of angular position will require increasing the gap, which may then extend beyond $3 \mathrm{~mm}$ - a power requirements and stability concern. It will also introduce mechanical oscillations and impose greater difficulty in maintaining control of axial position. For this application, two resolvers is a good solution, as they will likely only be about 0.5 inch in axial length and $\mathrm{x}$ and $\mathrm{y}$ position will be provided for both bearings 
In regards to temperature measurement, for $700^{\circ} \mathrm{C}$ continuous operation a type $\mathrm{N}$ thermocouple (TC) is suggested. The type $\mathrm{N}$ (nicrosil-nisil) thermocouples were developed as a lower drift alternative to other base metal (particularly Type K) thermocouples. ${ }^{34,35}$ Nicrosil and Nisil alloy compositions feature increased component solute concentrations (chromium and silicon) in the nickel base to transition from internal to surface modes of oxidation. They also include solutes (silicon and magnesium), which preferentially oxidize to form oxygen diffusion barriers. ${ }^{36}$ For temperatures to be measured near a high magnetic field (i.e., close to the stator windings), a type $S$ thermocouple is required. The type $S$ is composed of non-magnetic materials that are unaffected by the presence of magnetic fields and function well at $700^{\circ} \mathrm{C}$

\subsection{MOTOR CONCEPT}

Motor efficiency is strongly affected by the rotor-to-stator spacing because the amount of magnetic flux required to attract ferromagnetic material varies with the cube of the separation distance (characteristic of a dipole field). Designing the rotor and stator pole pieces to accommodate the separation is a principal motor design issue. Additionally, magnetic fields do not penetrate conductors instantaneously. The electrical conductivity of the can walls between the rotor and stator thus need to be minimized as well as their thickness. Enveloping the stator and its wiring within a shield avoids requiring sealed, multiconductor electrical penetrations for the stator electromagnet drives as well as chemical compatibility issues between the electromagnet winding insulation and stator itself with the working fluid.

The overall operating principle for a reluctance motor is that magnetic material when placed in a magnetic field will orient itself to minimize the reluctance of the magnetic circuit. When the rotor notches (between the poles) are opposite the stator poles, the magnetic circuit of the motor has a high magnetic reluctance. When the rotor poles are aligned with the stator poles, the magnetic circuit reluctance is minimized. When a stator pole pair is energized, the nearest rotor-pole pair will be pulled into alignment with the energized stator poles to minimize the overall motor reluctance. Motor rotation occurs through sequentially energizing the stator poles, causing the rotor to step to the next energized pole.

As the pump shaft needs to be positioned horizontally, vertically, and axially, the pump will have x-axis, $y$-axis, and z-axis bearings. We will denote the $\mathrm{x}$-axis and $\mathrm{y}$-axis as the radial axes and the $\mathrm{z}$-axis as the axial axis. To prevent the rotor from pivoting about the center of mass, two radial magnetic bearings will be used for the $\mathrm{x}$-axis and y-axis. Further, as the pump will need to accommodate start-up and shutdown, the pump will include mechanical touchdown bearings that will also serve as emergency catcher bearings to prevent pump damage in the event of loss of rotor control. Both graphite and silicon carbide are chemically compatible with fluoride salts. Hence, the catcher/touch-down bearing material will be fabricated from one of these materials.

An axial cross section of a pump design showing the cans, motor windings, and bearing windings is shown in Fig. 8. The initial design employs two radial magnetic bearings on either side of a switched reluctance motor, along with two radial magnetic bearings to position the shaft axially and counteract axial impeller forces. Mechanical catcher/touch-down bearings are provided at each end of the motor set as well. The rotor shaft is hollow to allow a fluid return path for the small amount of motor cooling flow through the gap between the rotor and stator cans.

\subsection{FAILURE MODES AND EFFECTS ANALYSIS}

FMEA is a systematic approach used to identify potential or known failure modes and provide corrective and preventive actions. This discipline was developed by the United States Military in $1949^{37}$ to determine the effect of system and equipment failures. The method is applicable to any system or equipment, at any desired level of design detail, and is essential in the development of any product. 
FMEA is a living document. Throughout the product development cycle, change and updates are made to the product and process. In fact these changes can and often do introduce new failure modes. It is therefore important to review and update the FMEA in special cases, for instance, when a new product or process is being initiated (at the beginning of the cycle) or when changes are made to the operating conditions in which the product or process is expected to function in (during the life cycle. The FMEA may be modified when a change is made to either the product or the process design. The product and process are interrelated since when the product design is changed the process is impacted and vice versa. ${ }^{38}$ The following diagram in Fig. 9 shows the basic development flow incorporating the FMEA.

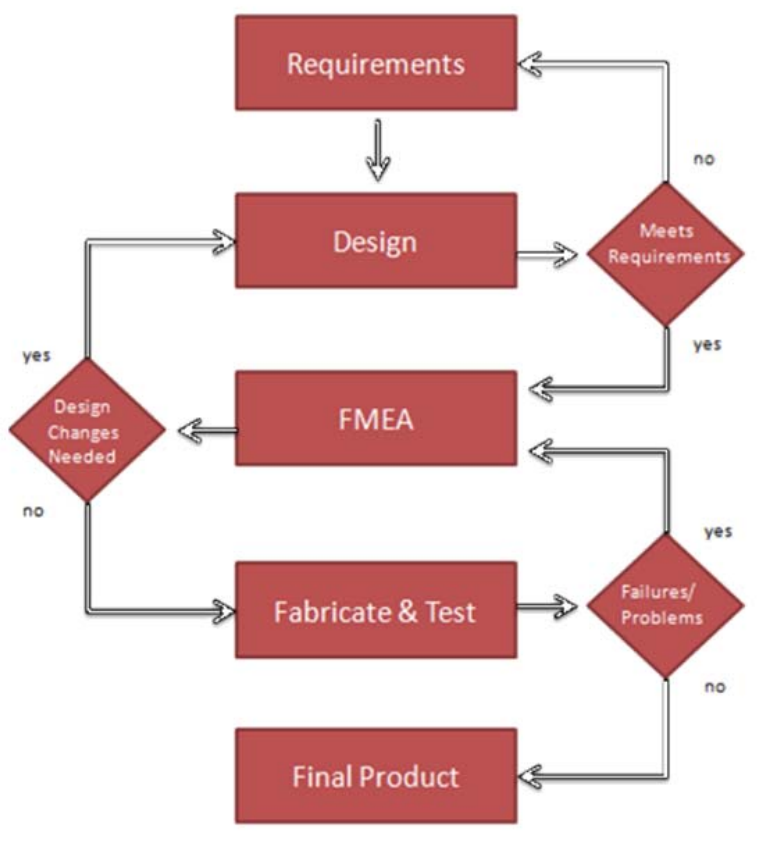

Fig. 9. System Development flow with FMEA.

The FMEA is multidisciplinary and used in the development of many products and systems. FMEA assists in selecting design alternatives with high reliability and high safety potential during early design phases. It ensures that all conceivable failure modes and their effects on the operational success of the system have been considered. By listing potential failures and identifying the magnitude of their effects, initial criteria can be developed for test planning and the design of test and checkout systems. FMEA provides a basis for quantitative reliability and availability analyses, input data for trade-off studies, a basis for establishing corrective action priorities, and historical documentation for future references to aid in the analysis of field failures and consideration of design changes. It also assists in the objective evaluation of design requirements related to redundancy, failure detection systems, fail-safe characteristics, and automatic and manual override. To facilitate a better understanding of how a FMEA is used, the template and definitions of the system are shown in Fig. 10. 


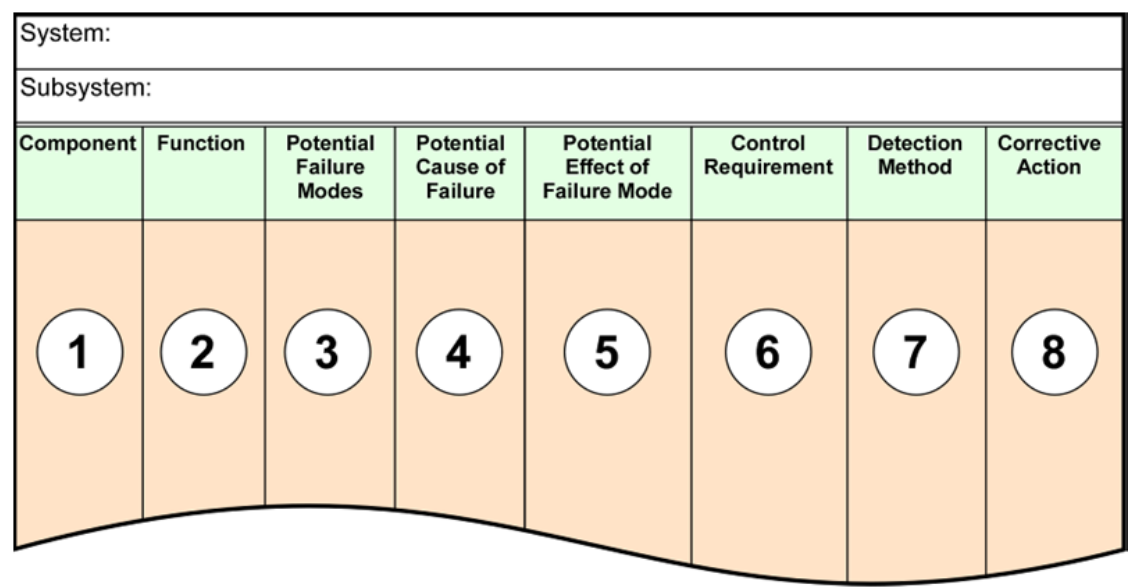

Fig. 10. FMEA template.

Columns of the template are defined as follows.

1. Component-This column identifies the element/part of the subsystem that is to be analyzed.

2. Function - In this column the objective, task, or purpose of the component is listed.

3. Failure Mode-The termination of the component's ability to perform a required function. Multiple failure modes are possible.

4. Cause of Failure Mode-The physical or chemical processes, design defects, quality defects, part misapplication, or other processes that are the basic reasons for failure or that initiate the physical process by which deterioration proceeds to failure.

5. Effect of Failure Mode-The consequence(s) a failure mode has on the operation, function, or status of system.

6. Control Requirement-How the current design is able to control the failure mode within the system.

7. Detection Method-The current design elements that could potentially detect the failure modes before occurrence.

8. Corrective Action-A documented design, process, procedure, or materials change to be put into consideration in order to correct the cause of failure or design deficiency.

An FMEA assessment was needed to facilitate a successful systems engineering approach for requirements and design due to the reliability and fault tolerance requirements and the engineering complexity of the embedded sensors and control system dictated by the testing platform. The FMEA process identifies possible failures, their probabilities, severity, and outcomes. This information is then used to direct engineering decisions to avoid, detect, and mitigate failure events. The FMEA is divided into three main systems - control, mechanical, and electrical - to differentiate and better account for components and aspects of the motor. Using this process led to emphasis on the aspects of the motor that would fail at a higher rate, and it identified extra precautions and embedded sensor requirements that could be considered. This information will be used to focus design decisions for sensing, failure detection, and control response.

For this project, the FMEA was divided into three main systems: control, mechanical, and electrical. Each subsystem was then broken down into individual components. This division was done in an effort to differentiate and better account for the different aspects of the motor so that embedded sensor selection and location decisions could be made. The component listing is shown in Table 4. 
Table 4. Canned rotor motor subsystem breakdown related to FMEA

\begin{tabular}{lll}
\hline \multicolumn{1}{c}{ Mechanical } & \multicolumn{1}{c}{ Electrical } & \multicolumn{1}{c}{ Control and sensor } \\
\hline - Axle & - Control power electronics & - Rotational position sensor \\
- Rotor laminations & - Power supplies & - Rotational speed sensor \\
- Bearing laminations & - Motor power electronics & - Axial position sensor \\
- Trapped gas inside rotor & - Windings & - Radial position sensor \\
- Rotor potting & - Wire leads & - Temperature sensors \\
- Rotor can & & - Vibration sensor \\
- FLiNaK & & - Flow meter \\
- Catcher bearings & & - Current sensor (on circuitry) \\
- Stator can & & - Analog input signal processing (opamps) \\
- Stator laminations & & - Analog to digital converter \\
- Stator potting & & - Software signal processing algorithms \\
- Stator coils (windings & & - Control computer \\
- Support structure & & - Poftware output signal conditioner \\
- Insulation & & \\
- Safety cage & & \\
- Impeller & & \\
- Impeller casing & & \\
- Bolts & & \\
- Seals (fixed) & & \\
\hline
\end{tabular}

This detailed breakdown helps show the complexity of the system and identify hundreds of failure modes. In Fig. 11, the basic flow of the control movement of the pump is shown. This figure helps identify the effects on the control system when failure precursors are sensed. 


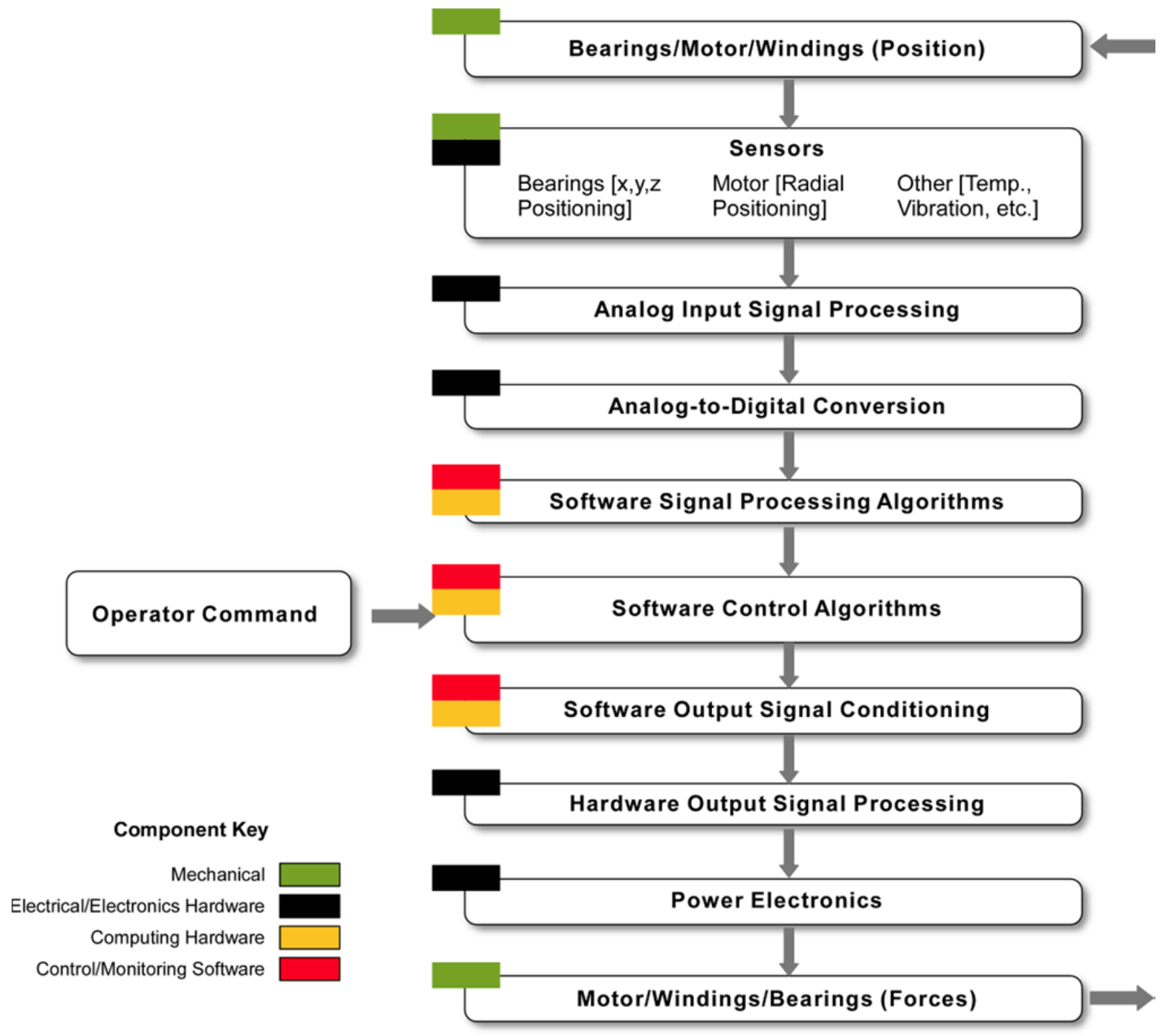

Fig. 11. Basic signal and information flow for pump control.

The use of the FMEA leads to consideration of many initial sensors and control system design decisions. Detailed FMEA results are found in Appendix A. Example considerations include the following:

- Routine start-up check for alignment and motor components placement

- Redundant sensors

- Diverse sensors (independent sensor and sensorless measurements)

- Redundant power for the magnetic suspension system

- Separate phase control for operation with phases missing

- Optimizing winding alignment for short circuit concerns including extra insulation between coil winding layers

- An overall "watch dog"

- Need for touch-down bearings

- Diagnostic and predictive software

- Corrosion estimation system

- Having a back-up motor replacement 


\section{THEORY DISCUSSION}

The following discussions are provided to establish the mathematical and logical basis for generating system requirements, developing the design, and eventually testing criteria. Topics covered are magnetic bearing control, bearing design, operation of SRMs, motor drive logic, heat transfer, and materials.

\subsection{SENSORLESS FEEDBACK MOTORS}

Variable speed drives, which are also called variable-frequency drives (VFDs), are utilized for applications such as pumps, fans, and industrial processes. Typical VFD control applications utilize feedback measurements or perform some form of sensorless control to regulate the torque for proper speed control. ${ }^{39}$ The feedback measurements typically consist of speed or rotational position. VFD control without measurement feedback consists of volts per hertz or flux vector control. Volts per hertz mode operates on the linear $\mathrm{V} / \mathrm{Hz}$ load relationship and is suitable for applications that tolerate poor speed regulation at low speed, poor dynamic speed regulation, and have nonvarying loads. Flux vector control mode operates by adjusting the voltage and frequency to achieve the desired speed through torque regulation and offers a significant improvement in speed and torque regulation versus V/Hz control. Flux Vector Control with rotational position feedback provides the high-speed regulation performance with typical speed regulation of 0.01 percent. ${ }^{39}$

Typical SRM systems require rotor angular position measurements in real time to properly coordinate the current waveforms for torque ripple minimization. ${ }^{14}$ The typical rotational position measurement for SRM systems is accomplished with an encoder; however, the canned rotor/stator does not enable with the use of encoders. Although angular position measurement can be implemented in this design with a resolver, sensorless techniques to obtain the rotor position using circuit measurements should be pursued to provide redundancy and to ensure error is not incurred from potential issues associated with the canned rotor. These sensorless techniques consist of voltage and current measurements at the motor terminals and derived parameters such as phase and inductance. ${ }^{13}$

SRM rotor sensorless measurements can be described in three broad classes:

1. observer-based schemes that estimate rotor position,

2. inductance-based measurements that use the winding current rise time and fall time to obtain discretely the rotor position,

3. inductance-based estimation of rotor position constant current or flux linkage applied to sensor signals and sensing phases. ${ }^{14}$

SRM sensorless methods can also be classified as the following.

1. Energized phase - Chopping waveform, regenerative current, flux-linkage, state observers, irregularities in stator/rotor poles, and current waveform methods

2. Un-energized phase - Active probing, modulated signal injection, regenerative current and mutually induced systems

3. Open loop methods - Dwell angle compensation and commutation angle compensation ${ }^{13}$

Inductive-based measurement systems have a three-dimensional relationship between inductance, current, and rotor position. Knowledge of current and inductance can facilitate resolving the rotor position. These sensorless techniques require high sample rate measurements and significant digital signal processing computation that may limit their application to lower motor speeds. Un-energized phase methods are only applicable at lower motor speeds, while energized phase methods are more applicable at high-speed operation. Most of the methods do not address the start-up rotor position, which may require some open- 
loop control during the start-up sequence. ${ }^{13}$ Other challenges with most sensorless control methods include the need for accurate voltage, inductance, and/or resistance measurements. Accurate measurement and integration of PWM voltage is challenging due to the limited bandwidth of most voltage transducers compared with the transients of the rising and falling PWM edges and switching oscillations that follow, which can be about $1 \mathrm{MHz}$. It is often assumed that inductance varies linearly from the unaligned to aligned position and that it does not vary significantly with current. Both of these assumptions will yield significant error in flux linkage estimation, and it is recommended that detailed inductance profiling be utilized instead. The impact of temperature upon winding resistance should also be carefully addressed.

\subsection{MAGNETIC BEARING CONTROL}

Magnetic bearings would not be possible without feedback control. Earnshaw's theorem states that it is impossible to levitate a configuration of magnets stably with another stationary configuration of magnets. This inherent instability requires active control to stabilize the system. In the following, the application of control techniques to active magnetic bearings is discussed. As a simple illustrative example, a model and controller are derived assuming a rigid rotor and independent axis control. The actual controller design for the demonstration platform will assume a flexible rotor and incorporate the dynamics of the sensors and electronic hardware and will not incorporate some of the simplifying assumptions presented in the following.

First, we will present background information and mathematical preliminaries for control systems. Let the non-forced linear differential equation

$$
\dot{x}=A x
$$

describe the behavior of a dynamical system where $x \in \mathbb{R}^{n}$ and $A \in \mathbb{R}^{n \times n}$. This system is stable if and only if $x(t) \rightarrow 0$ as $t \rightarrow \infty$. This statement is equivalent to the eigenvalues of $A$ being in the open left half plane. Feedback control requires the addition of sensors to measure the current state of the system (measured output) and actuators to effect the state of the system (control inputs). This leads to the state space formulation of the system dynamics

$$
\begin{aligned}
& \dot{x}=A x+B u \\
& y=C x+D u
\end{aligned}
$$

where $x \in \mathbb{R}^{n}, u=\in \mathbb{R}^{m}, y=\in \mathbb{R}^{l}, A \in \mathbb{R}^{n \times n}, B \in \mathbb{R}^{n \times m}, C \in \mathbb{R}^{l \times n}$, and $D \in \mathbb{R}^{l \times m}$. The controlled system is referred to as the plant.

Next, we present a simple model of the rotor as a rigid body. The system will be linearized about the center of mass, as shown in Fig. 12, which simplifies the model by decoupling the axial rotation $\Omega$ from the other two Euler angles $\alpha$ and $\beta$. This yields the system state vector $x=\left[\beta, x_{s},-\alpha, y_{s}\right]^{T}$. 


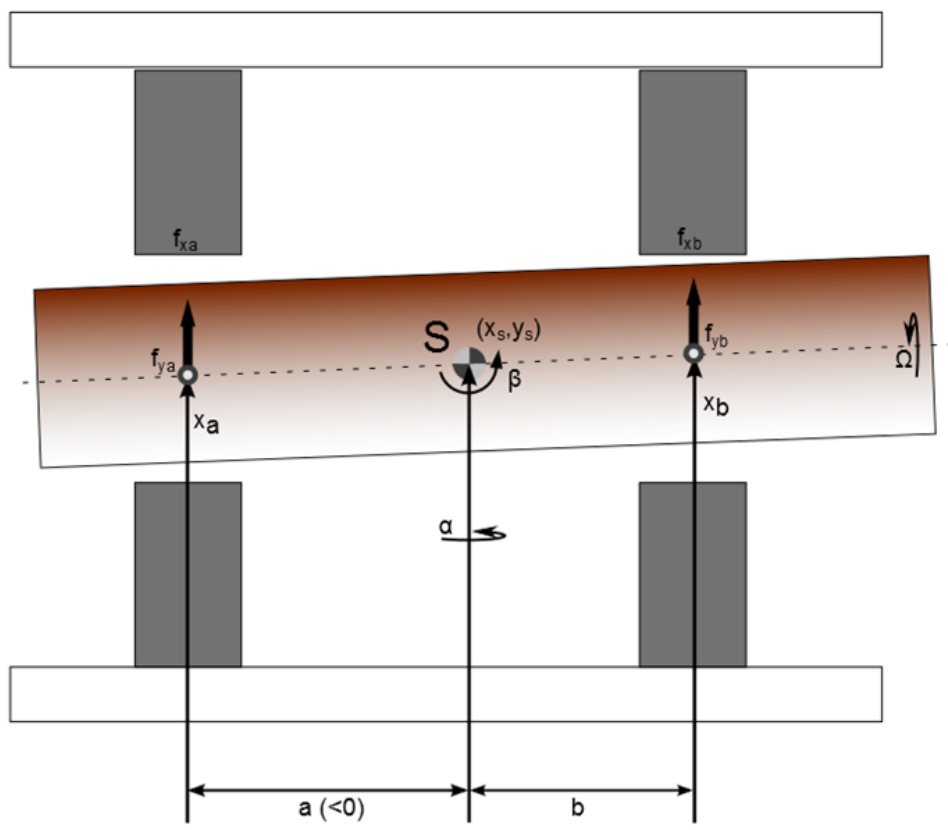

Fig. 12. Inertial coordinate system for rotor.

The measured outputs are the positions of the axis of the rotor at each magnetic bearing $y=\left[x_{a}, x_{b}, y_{a}, y_{b}\right]^{T}$. The system inputs are given by the radial magnetic bearing forces $u=\left[f_{x a}, f_{x b}, f_{y a}, f_{y b}\right]^{T}$. This approach yields a state-space formulation for the rotor dynamics given by the following matrix differential equation

$$
\begin{aligned}
& M \ddot{x}+G \dot{x}=B u \\
& y=C x
\end{aligned}
$$

where

$$
\begin{aligned}
& M=\left[\begin{array}{cccc}
I_{y} & 0 & 0 & 0 \\
0 & m & 0 & 0 \\
0 & 0 & I_{x} & 0 \\
0 & 0 & 0 & m
\end{array}\right], G=\left[\begin{array}{cccc}
0 & 0 & I_{z} \Omega & 0 \\
0 & 0 & 0 & 0 \\
-I_{z} \Omega & 0 & 0 & 0 \\
0 & 0 & 0 & 0
\end{array}\right], \\
& B=\left[\begin{array}{llll}
a & b & 0 & 0 \\
1 & 1 & 0 & 0 \\
0 & 0 & a & b \\
0 & 0 & 1 & 1
\end{array}\right], \quad C=\left[\begin{array}{llll}
a & 1 & 0 & 0 \\
b & 1 & 0 & 0 \\
0 & 0 & a & 1 \\
0 & 0 & b & 1
\end{array}\right]
\end{aligned}
$$

An output feedback controller is given by the following dynamical system

$$
u=K y
$$


where $K \in \mathbb{R}^{m \times l}$. The objective is to derive a control matrix $K$ that stabilizes the system around the nominal operating state $x=0$ that provides the necessary performance. There are numerous options for the design of the controller. One of the most easily implementable is linear quadratic Gaussian (LQG) control, which is optimal in a strict sense but sensitive to uncertainties in system parameters. This approach does not require full state feedback because of the presence of a state estimator. Another technique is passivity-based control. This type of controller is robust to model uncertainties but sensitive to nonlinearities in sensors and the plant. Another possibility for control system design is robust control, otherwise known as $\mathrm{H}_{\infty}$ or $\mu$-synthesis. Robust control is well suited to complex applications such as active magnetic bearings and is robust to system uncertainties. Controllers designed through robust techniques are frequently high order and require more computational power to implement. The simplest method for controller design is PID control. This method is well understood and easy to implement; however, it cannot achieve the robustness and performance of other methods.

As an example, we derive a simple PD, non-collocated controller. To begin the controller derivation, one looks at the relationship between the current, position, and force on the rotor. The control action will be a linear combination of current and position given by

$$
\begin{aligned}
u & =\left[\begin{array}{l}
f_{x a} \\
f_{x b} \\
f_{y a} \\
f_{y b}
\end{array}\right]=-\left[\begin{array}{cccc}
k_{s a} & 0 & 0 & 0 \\
0 & k_{s b} & 0 & 0 \\
0 & 0 & k_{s c} & 0 \\
0 & 0 & 0 & k_{s d}
\end{array}\right]\left[\begin{array}{l}
x_{a} \\
x_{b} \\
y_{a} \\
y_{b}
\end{array}\right]+\left[\begin{array}{cccc}
k_{i a} & 0 & 0 & 0 \\
0 & k_{i b} & 0 & 0 \\
0 & 0 & k_{i c} & 0 \\
0 & 0 & 0 & k_{i d}
\end{array}\right]\left[\begin{array}{l}
i_{x a} \\
i_{x b} \\
i_{y a} \\
i_{y b}
\end{array}\right] \\
u & =-K_{s} y+K_{i} i
\end{aligned}
$$

The stiffness matrix $K_{s}$ and current matrix are linearized about the operating point. Examples of the relationship between force/displacement and force/current are shown in Figs. 13 and 14 for one coil. 


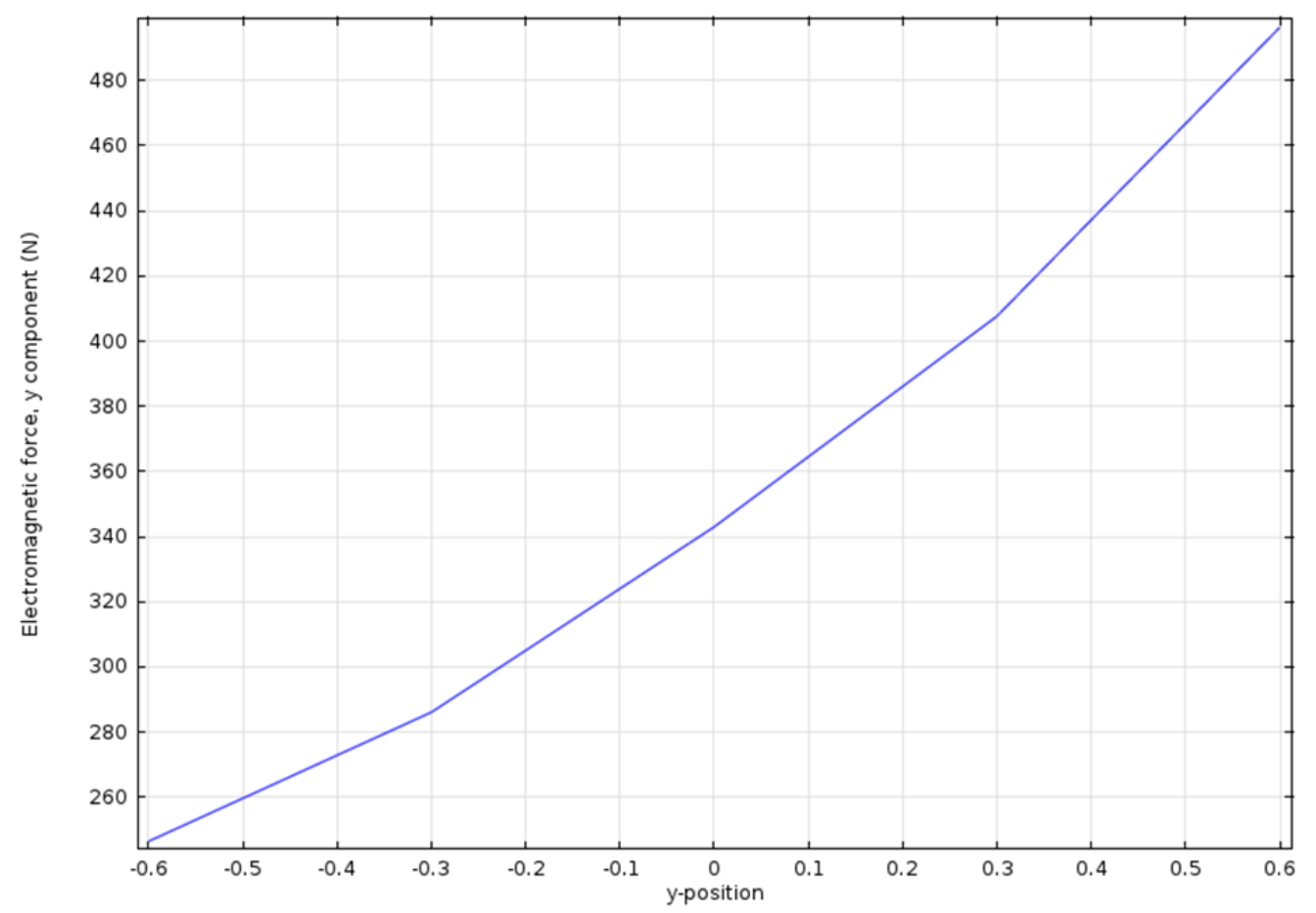

Fig. 13. Simulation showing the rotor force with a $10 \mathrm{~A}$ coil current and varying position from $-0.6 \mathrm{~mm}$ to $0.6 \mathrm{~mm}$.

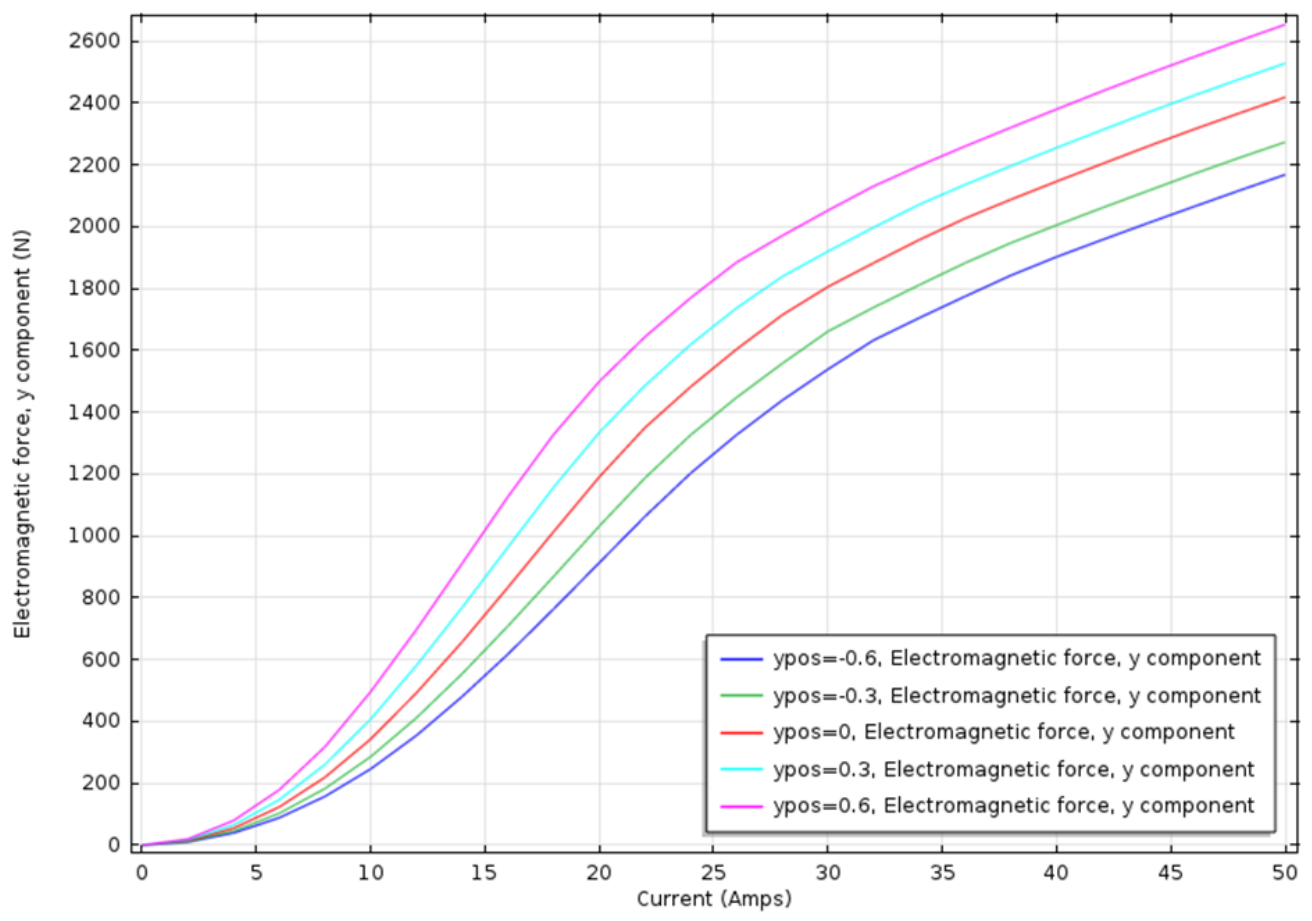

Fig. 14. Current force relationship for varying rotor positions. 
This yields the linearized model given by

$$
\begin{aligned}
& M \ddot{x}+G \dot{x}=B\left(-K_{s} y+K_{i} i\right) \\
& y=C x
\end{aligned}
$$

Let $K_{s S}=B K_{s} C$, then the equation of motion becomes

$$
M \ddot{x}+G \dot{x}+K_{s S} x=B K_{i} i
$$

Transforming the force/displacement vector to the center of mass coordinate system yields a system that is dependent only on the current as the control input $i \in \mathbb{R}^{4}$. Note that the position measurement at each bearing is still necessary in the feedback control law, as shown in the following. Given a matrix differential equation of the following form in Equation (9),

$$
\begin{aligned}
& M \ddot{x}+G \dot{x}+K x=B i \\
& y=C x
\end{aligned}
$$

Equation (9) can be transformed into the state-space form using Equation (10):

$$
\begin{aligned}
& {\left[\begin{array}{c}
\dot{x} \\
\ddot{x}
\end{array}\right]=\left[\begin{array}{cc}
0 & I \\
-M^{-1} K & -M^{-1} G
\end{array}\right]\left[\begin{array}{l}
x \\
\dot{x}
\end{array}\right]+\left[\begin{array}{c}
0 \\
M^{-1} B
\end{array}\right] i} \\
& y=\left[\begin{array}{ll}
C & 0
\end{array}\right]\left[\begin{array}{c}
x \\
\dot{x}
\end{array}\right]
\end{aligned}
$$

Applying this transformation to the active magnetic bearing system given by Equation (7) yields

$$
\begin{aligned}
& {\left[\begin{array}{l}
\dot{x} \\
\ddot{x}
\end{array}\right]=\left[\begin{array}{cc}
0 & I \\
-M^{-1} B K_{s} C & -M^{-1} G
\end{array}\right]\left[\begin{array}{l}
x \\
\dot{x}
\end{array}\right]+\left[\begin{array}{c}
0 \\
M^{-1} B K_{i}
\end{array}\right] i} \\
& y=\left[\begin{array}{ll}
C & 0
\end{array}\right]\left[\begin{array}{l}
x \\
\dot{x}
\end{array}\right]
\end{aligned}
$$

The system model of Equation (11) can also be formulated in the frequency domain. Taking the Laplace transform of the state-space system given in Equation (2) yields the input/output transfer function given by Equation (12):

$$
G(s)=\frac{Y(s)}{U(s)}=C(s I-A)^{-1} B+D
$$

As an illustrative example, a simple proportional/derivative (PD) controller is derived with each bearing axis controlled independently. This control is adequate for a theoretical discussion, but the assumptions required for this type of control to be stable under a wide range of operating conditions are inadequate. In reality, due to gyroscopic effects, manufacturing defects, and the non-collocation of the x-axis and y-axis, an axis independent controller will be unstable at certain rotational speeds. The non-collocation assumption leads to a feedback control law given by 


$$
i=-\left(K_{p} y+K_{D} \dot{y}\right)
$$

where $P$ and $D$ are diagonal matrices with the proportional and derivative gains for each of the four control axes. Combined with the equation of motion [Equation (8)], the closed-loop system in the center of mass coordinate system becomes

$$
M \ddot{x}+G \dot{x}+K_{s S} x=-B K_{i}\left(K_{P} C x+K_{d} C \dot{x}\right)
$$

Rearranging the terms to the left hand side of the equation yields

$$
M \ddot{x}+G \dot{x}+K_{s S} x+B K_{i} K_{p} C x+B K_{i} K_{D} C \dot{x}=0
$$

The stiffness matrix $K_{c}=B K_{i} K_{P} C$ and the damping matrix $D_{c}=B K_{i} K_{D} C$ are designed so that the closed-loop system is stable; that is, the closed-loop eigenvalues $\lambda$ given by the solutions to $\operatorname{det}(\lambda I-A)=0$ are in the open left-half plane. By transforming Equation (15) into state-space form, the corresponding closed-loop equations of motion become

$$
\begin{aligned}
& \dot{x}=A x \\
& A=\left[\begin{array}{cc}
0 & I \\
-M^{-1}\left(K_{s S}+K_{c}\right) & -M^{-1}\left(G+D_{c}\right)
\end{array}\right]
\end{aligned}
$$

On a side note, the lack of an integral term in the controller will lead to the system having a bias error.

\subsection{RELUCTANCE MOTORS}

Electric machine (motor) types can be categorized into broad classes of synchronous and asynchronous, depending on whether or not the rotor mechanical speed is some integral submultiple of the stator electrical excitation frequency. Synchronous motors all possess rotor speeds that rotate at $2 / \mathrm{P} * \omega_{\mathrm{e}}$, where $\mathrm{P}$ $=$ number of poles and $\omega_{\mathrm{e}}$ is the electrical excitation frequency of the applied stator currents.

Asynchronous motors rely on electrical induction to generate current flow in the rotor conductors (cage or wound type) that in turn react with the stator currents that produced them to develop electromagnetic torque. The major types of electrical machines are shown in Fig. 15. 


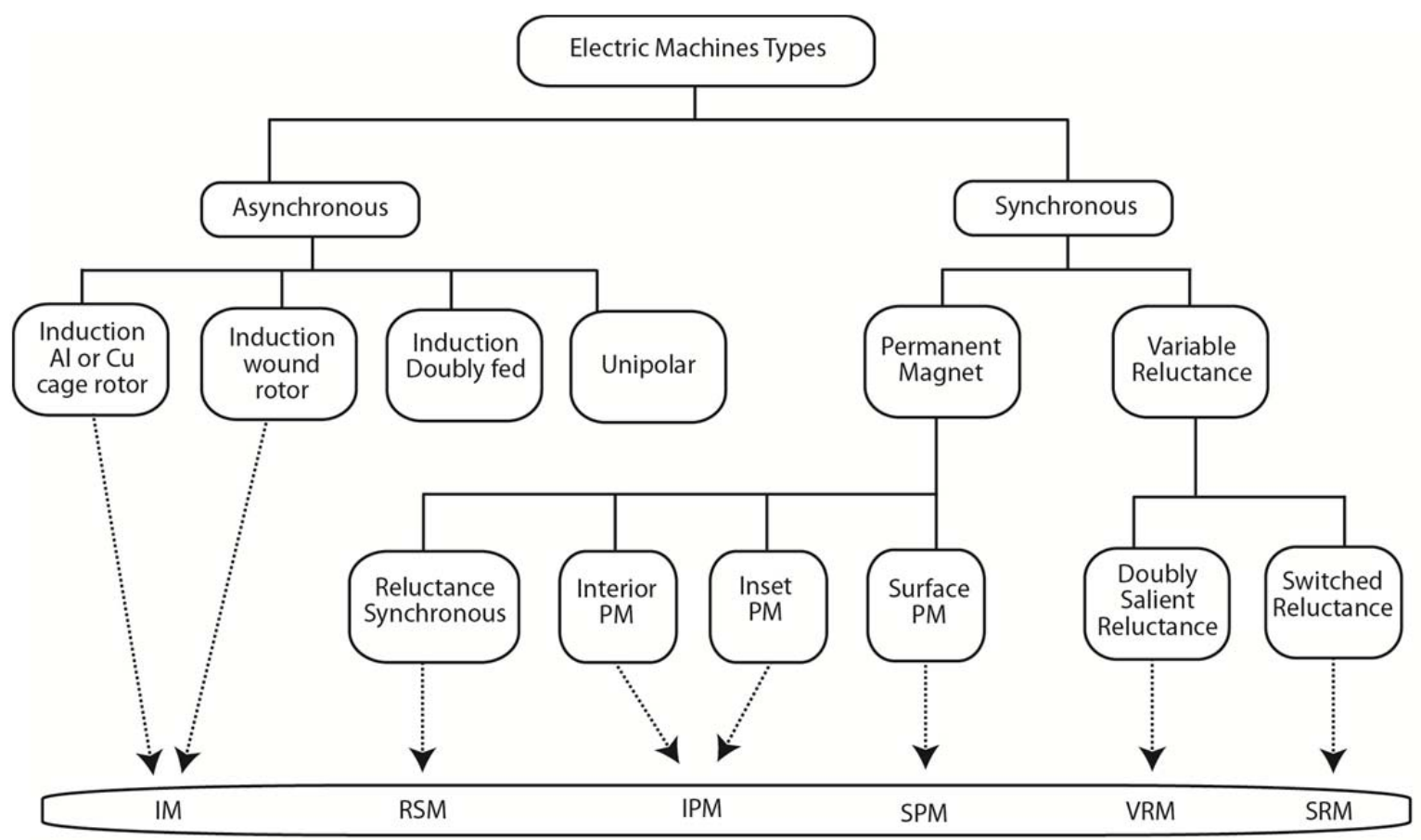

Fig. 15. Classification of major electric motor types.

Cross sections of the most representative electric machines are shown in cross section in the Fig. 16 and explained in more detail in Table 5. A comparison of three motor types is shown in Table 6 . The conclusion is that the best choice for a canned (no rotating seals) motor to operate at high temperatures is the reluctance motor. 


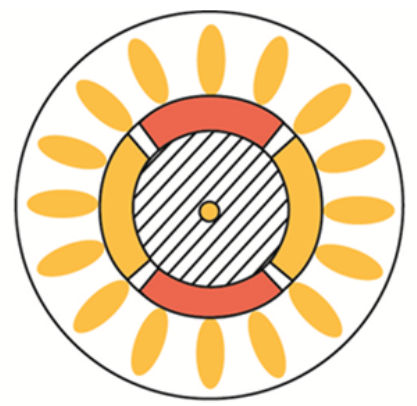

A) Surface Permanent Magnet SPM

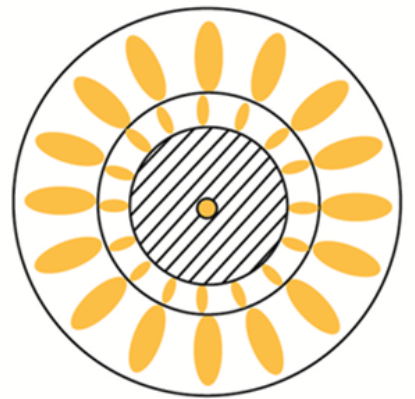

C) Induction Machine, IM

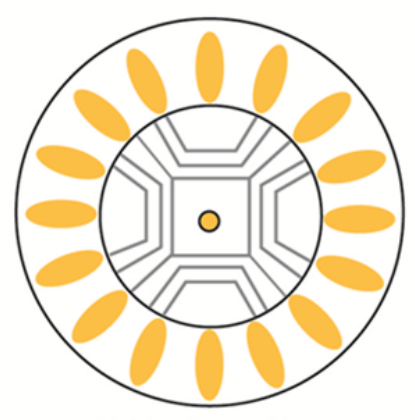

E) Reluctance Sync

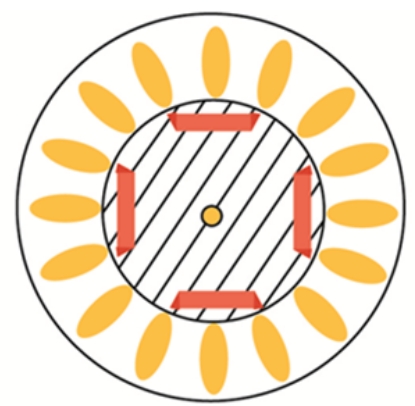

B) Interior Permanent Magnet IPM

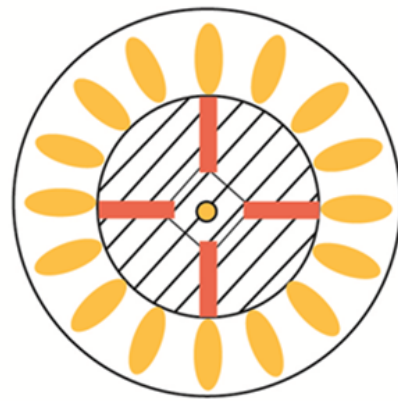

D) Interior PM - flux squeeze

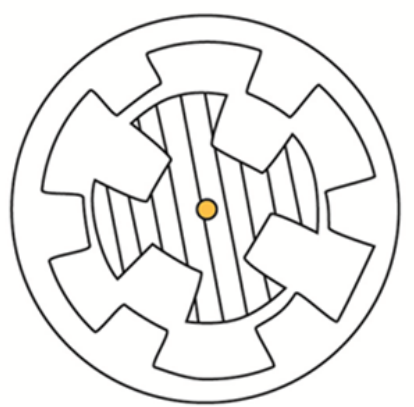

F) Variable Reluctance Machine VRM

Fig. 16. Electric motors shown in cross section by major type. 
Table 5. Comparison of motor types

\begin{tabular}{|c|c|c|c|c|}
\hline Motor type & Advantages & Disadvantages & Typical applications & Typical drive \\
\hline $\begin{array}{l}\text { ac polyphase } \\
\text { induction squirrel } \\
\text { cage }\end{array}$ & $\begin{array}{l}\text { Low cost, long life, } \\
\text { high efficiency, high } \\
\text { starting torque, large } \\
\text { power ratings } \\
\text { available }(>1 \mathrm{MW}), \\
\text { large selection of } \\
\text { standardized types }\end{array}$ & $\begin{array}{l}\text { High starting inrush } \\
\text { current, speed control } \\
\text { requires variable } \\
\text { frequency/voltage } \\
\text { source, speed slightly } \\
\text { below synchronous }\end{array}$ & $\begin{array}{l}\text { Pumps, fans, blowers, } \\
\text { conveyors, } \\
\text { compressors }\end{array}$ & $\begin{array}{l}\text { Poly-phase ac, } \\
\text { variable- } \\
\text { frequency ac }\end{array}$ \\
\hline $\begin{array}{l}\text { ac induction-- } \\
\text { Squirrel cage, } \\
\text { split-phase } \\
\text { capacitor-start }\end{array}$ & $\begin{array}{l}\text { High power, high } \\
\text { starting torque }\end{array}$ & $\begin{array}{l}\text { Speed slightly below } \\
\text { synchronous, starting } \\
\text { switch or relay required }\end{array}$ & $\begin{array}{l}\text { Appliances, stationary } \\
\text { power tools }\end{array}$ & Single-phase ac \\
\hline $\begin{array}{l}\text { ac induction - } \\
\text { squirrel cage, } \\
\text { split-phase } \\
\text { capacitor-run }\end{array}$ & $\begin{array}{l}\text { Moderate power, high } \\
\text { starting torque, no } \\
\text { starting switch, } \\
\text { comparatively long } \\
\text { life }\end{array}$ & $\begin{array}{l}\text { Speed slightly below } \\
\text { synchronous, slightly } \\
\text { more costly than } \\
\text { capacitor start }\end{array}$ & $\begin{array}{l}\text { Industrial blowers, } \\
\text { industrial machinery }\end{array}$ & Single-phase ac \\
\hline $\begin{array}{l}\text { ac induction - } \\
\text { squirrel cage } \\
\text { motor, split- } \\
\text { phase, auxiliary } \\
\text { start winding }\end{array}$ & $\begin{array}{l}\text { Moderate power, low } \\
\text { starting torque }\end{array}$ & $\begin{array}{l}\text { Speed slightly below } \\
\text { synchronous tarting } \\
\text { switch or relay required }\end{array}$ & $\begin{array}{l}\text { Appliances, stationary } \\
\text { power tools }\end{array}$ & Single-phase ac \\
\hline $\begin{array}{l}\text { Shaded-pole } \\
\text { motor }\end{array}$ & Low cost, long life & $\begin{array}{l}\text { Speed slightly below } \\
\text { synchronous, very low } \\
\text { starting torque, small } \\
\text { ratings, very low } \\
\text { efficiency }\end{array}$ & Fans, appliances & Single-phase ac \\
\hline Universal motor & $\begin{array}{l}\text { High starting torque, } \\
\text { compact, high speed }\end{array}$ & $\begin{array}{l}\text { Maintenance (brushes } \\
\text { wear), shorter lifespan, } \\
\text { often acoustically noisy, } \\
\text { only small ratings }\end{array}$ & $\begin{array}{l}\text { Handheld power tools, } \\
\text { kitchen appliances, } \\
\text { vacuum cleaners, some } \\
\text { blowers }\end{array}$ & $\begin{array}{l}\text { Single-phase ac } \\
\text { or dc }\end{array}$ \\
\hline ac Synchronous & Synchronous speed & $\begin{array}{l}\text { More costly than ac } \\
\text { polyphase induction } \\
\text { squirrel cage }\end{array}$ & $\begin{array}{l}\text { Industrial motor, } \\
\text { clocks, volt-amperes } \\
\text { reactive (VAR) control }\end{array}$ & $\begin{array}{l}\text { Single- or } \\
\text { Polyphase-ac } \\
\text { (capacitor-run } \\
\text { for single-phase } \\
\text { ac) }\end{array}$ \\
\hline Stepper dc & $\begin{array}{l}\text { Precision positioning, } \\
\text { high holding torque }\end{array}$ & $\begin{array}{l}\text { Can be costly, requires a } \\
\text { controller and power } \\
\text { drive }\end{array}$ & $\begin{array}{l}\text { Positioning in hard } \\
\text { drives, industrial } \\
\text { machine tools, printers }\end{array}$ & dc-pulsed \\
\hline Brushless dc & $\begin{array}{l}\text { Long lifespan, low } \\
\text { maintenance, high } \\
\text { efficiency }\end{array}$ & $\begin{array}{l}\text { Higher initial cost, } \\
\text { requires a controller and } \\
\text { drive electronics }\end{array}$ & $\begin{array}{l}\text { Hard disk drives, } \\
\text { CD/DVD players, } \\
\text { electric vehicles, } \\
\text { remote control } \\
\text { Vehicles, UAVs }\end{array}$ & dc or PWM \\
\hline $\begin{array}{l}\text { Switched } \\
\text { reluctance motor }\end{array}$ & $\begin{array}{l}\text { Long lifespan, low } \\
\text { maintenance, high } \\
\text { efficiency, no } \\
\text { permanent magnets }\end{array}$ & $\begin{array}{l}\text { Requires a controller and } \\
\text { drive electronics }\end{array}$ & $\begin{array}{l}\text { Appliances, electric } \\
\text { vehicles, textile mills, } \\
\text { aircraft applications }\end{array}$ & dc or PWM \\
\hline
\end{tabular}


Table 5. Comparison of motor types (continued)

\begin{tabular}{cllll}
\hline Motor Type & \multicolumn{1}{c}{ Advantages } & \multicolumn{1}{c}{ Disadvantages } & Typical applications & Typical drive \\
\hline Brushed dc & $\begin{array}{l}\text { Simple speed control, } \\
\text { very high torque }\end{array}$ & $\begin{array}{l}\text { Maintenance (brushes } \\
\text { wear), medium lifespan, } \\
\text { costly commutator and } \\
\text { brushes }\end{array}$ & $\begin{array}{l}\text { Steel mills, paper } \\
\text { making machines, } \\
\text { exercise equipment, } \\
\text { automotive accessories }\end{array}$ & $\begin{array}{l}\text { Direct dc or } \\
\text { PWM }\end{array}$ \\
& & $\begin{array}{l}\text { Medium cost, medium } \\
\text { lifespan }\end{array}$ & $\begin{array}{l}\text { Office equipment, fans, } \\
\text { pumps, fast industrial } \\
\text { and military servos }\end{array}$ & PWM \\
& $\begin{array}{l}\text { Compact design, } \\
\text { simple speed control }\end{array}$ & & & \\
& & & &
\end{tabular}

Table 6. Comparison of three motor types relative to high-temperature applications

\begin{tabular}{|c|c|c|c|}
\hline & $\begin{array}{c}\text { Permanent } \\
\text { Magnet Motor }\end{array}$ & $\begin{array}{l}\text { Induction } \\
\text { Motor }\end{array}$ & $\begin{array}{c}\text { Reluctance } \\
\text { Motor }\end{array}$ \\
\hline High temperature operation & Poor & Moderate & Better \\
\hline Starting torque in hot ambient & Minimal & Good & Easy \\
\hline Suited to large gap designs & Best & Moderate & Moderate \\
\hline Suited to canned rotor and stator & Worst & Poor & Better \\
\hline Efficiency (\%) in hot environment & Poor & Moderate & Better \\
\hline Noise and vibration produced & Moderate & Best & Better \\
\hline Manufacturability & Difficult & Mature & Easiest \\
\hline Suited to sensorless position control & Significant & Minimal & Significant \\
\hline
\end{tabular}

\subsection{MOTOR DRIVE}

The SRM drive consists of insulated-gate bipolar transistor (IGBT) power stage, gate drivers, phase current sensing, a digital controller, and provision for rotor position sensing (rps). The main power stage of the SRM inverter houses the high-voltage (HV) dc link buss bars and filter capacitors needed to maintain voltage stability and minimize transient overvoltage to the IGBT power module. Figure 17 illustrates the 6/4 SRM architecture for 3-phase inverter excitation to the stator and the $\mathrm{Nr}=$ four pole rotor. In the pump application, the rotor material is housed in a Hastelloy ${ }^{\circledR} \mathrm{N}$ can that will spin suspended in the molten salt being pumped. The stator poles are shielded from this salt by another Hastelloy ${ }^{\circledR} \mathrm{N}$ can for a combined gap of $3 \mathrm{~mm}$ total. The power driver consists of an upper IGBT that connects to the positive dc rail (approx. $325 \mathrm{~V} \mathrm{dc}$ ) and to a phase winding start. The phase winding itself consists of two coils, one each along a stator diagonal, so that the phase winding finish connects to the power inverter lower IGBT. Gate drivers on each IGBT interface the power stage to a controller electronics module. SRM control consists of phase steering signals to the upper IGBTs and phase current modulation signals to the lower IGBTs. The phase steering signals sequence the stator phase coil current in relation to the rps signal so that current builds in the phase coils during an interval when a rotor pole pair are unaligned to 
the phase. Pulse width modulation (PWM) is used to regulate the SRM stator current to the required level for specific torque production.
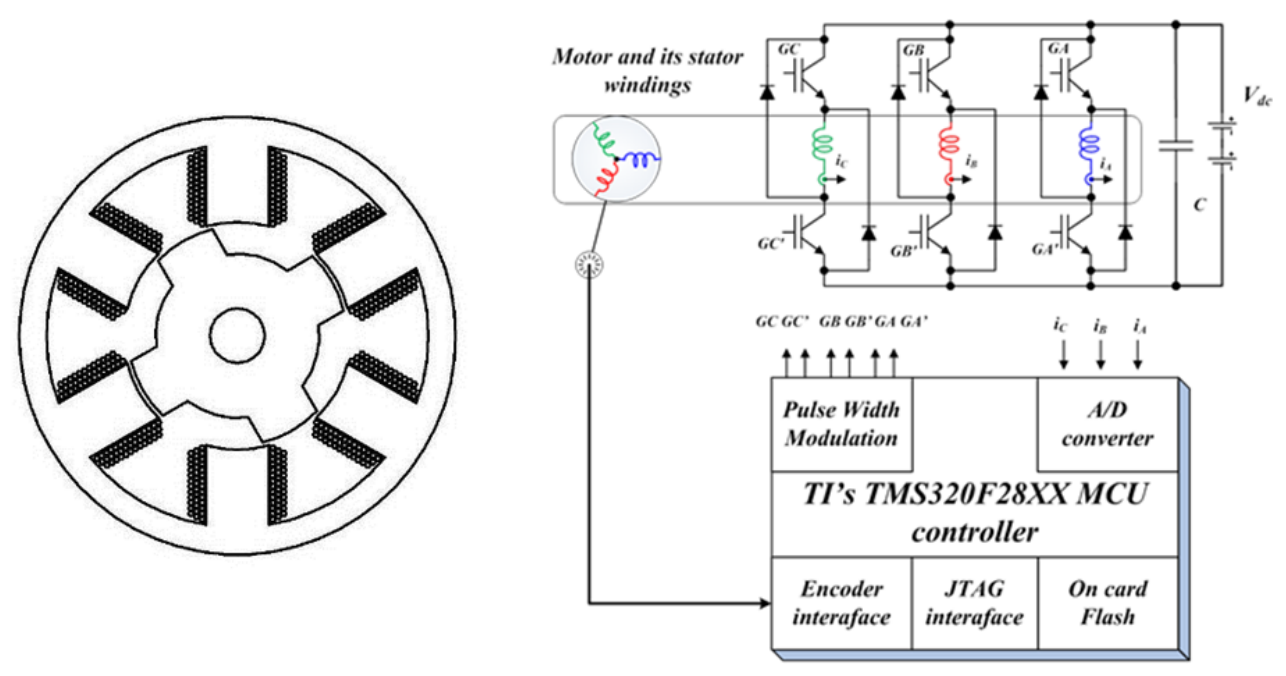

Fig. 17. The 6/4 SRM baseline geometry and associated power electronics stage.

For an SRM, and for any pole number combination, the rotor step angle, $\theta_{\text {step }}$, is given as Equation (17). In a $6 / 4 \mathrm{SRM}$, the step angle $\theta_{\text {step }}=30^{\circ}$ (also found as $\theta_{\text {step }}=360 / \mathrm{m}^{*} \mathrm{~N}_{\mathrm{r}}$ ), and for m-phase machine, $\mathrm{m} \theta_{\text {step }}=\theta_{\mathrm{r}}=360 / \mathrm{N}_{\mathrm{r}}$. The rps sensor (encoder, resolver, or indirect sensing) needs to be accurate to within 0.3 degree electrical $\left(\theta_{\mathrm{r}} / \theta_{\text {step }} * 0.1\right.$ degree mechanical $)$, or $\mathrm{N}=\operatorname{Int}\{11.8\}=12$ bits.

$$
\theta_{\text {step }}=\frac{\left|N_{s}-N_{r}\right|}{N_{s} N_{r}} 360^{\circ}
$$

The SRM control requires accurate resolution of the rotor angle during normal operation and for phase advance at higher operating speeds. Representative waveforms for an SRM in normal operation are shown in Fig. 18 for illustration. The SRM controller must synthesize the correct current profile to match SRM torque and speed requirements via appropriate PWM control of the phase switches shown in Fig. 20. The electrical frequency of the 6/4 SRM, Equation (18), is 12 times the mechanical (rotor) frequency (i.e., the $6 / 4$ is a 12 pulse/rev machine).

$$
f_{e}=m N_{r} f_{m}
$$

The total time for one phase pulse interval is therefore $1 /\left(\mathrm{m} \mathrm{N}_{\mathrm{r}} \mathrm{f}_{\mathrm{e}}\right)$ seconds, and rate $\mathrm{d} \theta / \mathrm{dt}=\mathrm{m} \mathrm{N} \mathrm{N}_{\mathrm{r}} \mathrm{f}_{\mathrm{r}}$ $(\mathrm{deg} / \mathrm{sec})$. In the phase current illustration shown in Fig. 20, the phase excitation rate is $22500^{\circ} / \mathrm{sec}$, or $22.5^{\circ} / \mathrm{ms}$. In this illustration, the phase current dwell is approximately $36^{\circ}$. 

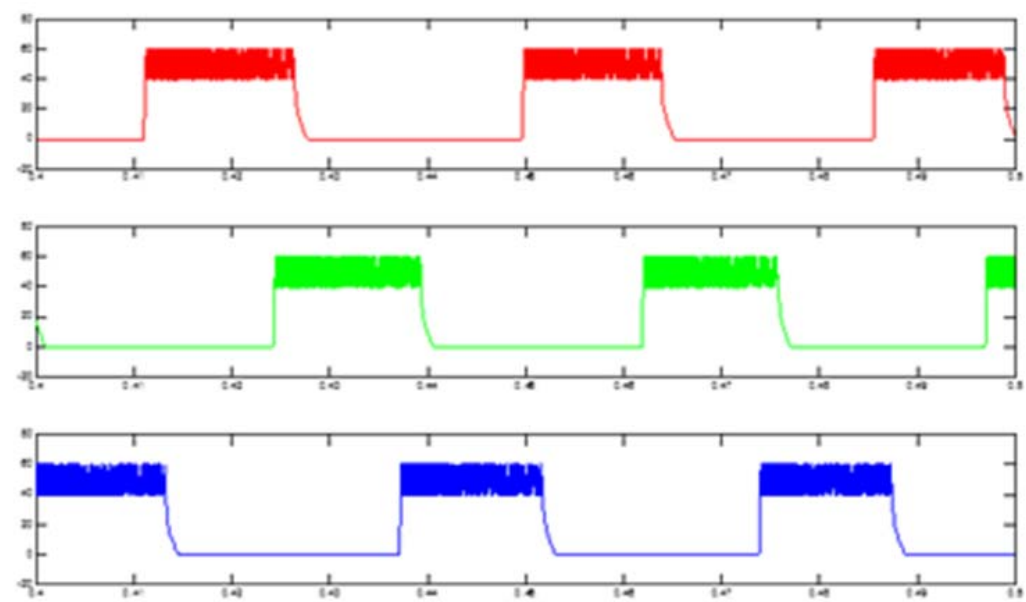

Fig. 18. Representative 6/4 SRM phase current waveforms at n 312 rpm with phase turn-on angle $\theta_{\mathrm{ON}}=40^{\circ}$ and turn-off angle $\theta_{\mathrm{OFF}}=7^{\circ}$.

\subsubsection{Normal operations}

The SRM pump motor will be required to use a nickel alloy (Hastelloy ${ }^{\circledR} \mathrm{N}$ or alternative) cans to protect the rotor outer diameter (OD) and stator inner diameter (ID). For example, the high-temperature SRM requires a $1 \mathrm{~mm}$ thick can fixed to the inner bore of the stator to shield it from the molten fluid as shown in Fig. 19. Similarly, the rotor itself must have its ferrous laminations shielded from the molten fluid, only here the approximately $1 \mathrm{~mm}$ thick can rotates with the rotor through the molten fluid.
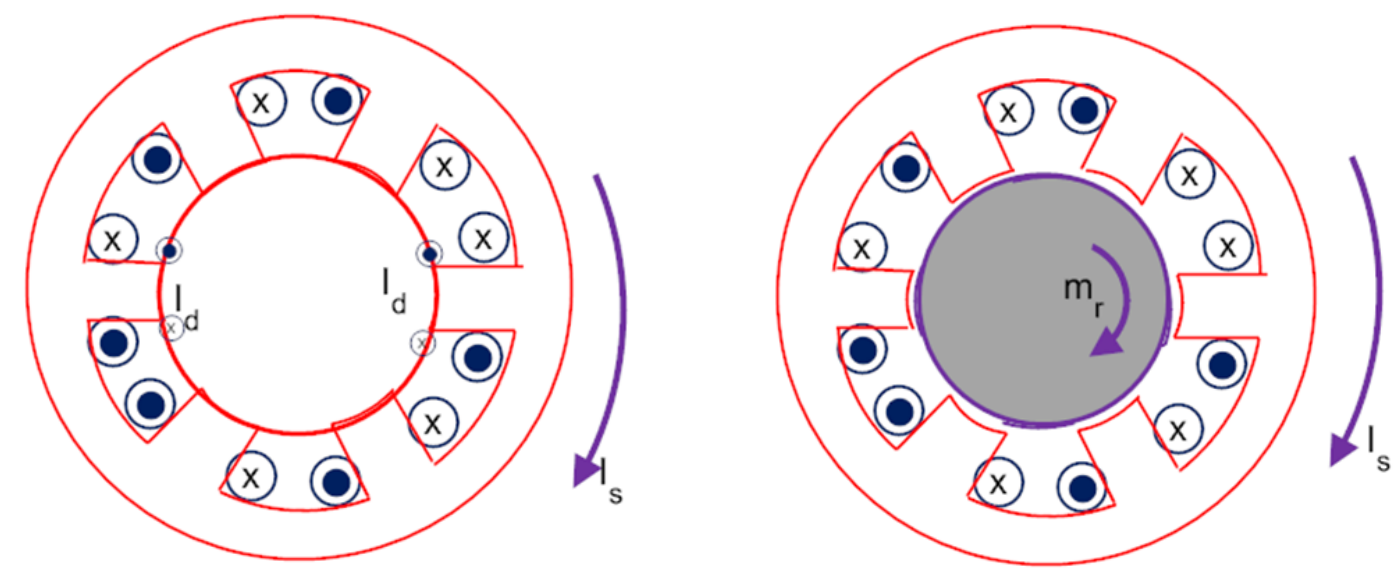

Fig. 19. Representative 6/4 SRM. Left: stator assembly with Hastelloy ${ }^{\circledR}$ can. Right: rotor assembly with Hastelloy ${ }^{\circledR}$ can fixed to the rotor.

The phase currents shown in Fig. 18 are applied to the phase windings on stator poles shown in the left graphic in Fig. 19, where phase A is the horizontal pole pair, phase B the diagonal pole pair in the $-60^{\circ}$ position, and phase $\mathrm{C}$ in the $-120^{\circ}$ position. When the stator is excited in phase sequence A-B-C according to Fig. 18, the magnetic flux is similar to that of a two-pole synchronous machine. However, the presence of the conducting can in the stator bore acts as a damper winding in a synchronous machine. This behavior is captured by currents $I_{d}$ in the can at the phase-A pole faces. These eddy currents act to dampen any rapid buildup of flux and, if the conducting layer is sufficiently thick, will limit the response. 
The sequencing of stator currents in the SRM is depicted as the rotational symbol, $\mathrm{I}_{\mathrm{s}}$. A similar situation exists at the rotor due to the presence of a conducting can with ferrous interior. The rotor saliencies are omitted for clarity in Fig. 19 in order to make the following point. A rotating two-pole stator magnetic field rotating through a conducting can is essentially an asynchronous electric machine, that is, an induction motor. The eddy currents in the rotor lag the stator field due to conductivity of the material and thereby interact with the stator currents, producing an electromagnetic torque on the can shown as rotor moment, $\mathrm{m}_{\mathrm{IM}}$, for induction motor effect, in Fig. 19. Notice that this torque is in the direction of the stator excitation sequence, $\mathrm{I}_{\mathrm{s}}$. It is understood that rotation in a doubly salient electric machine is counter to the excitation sequence, and therefore the intended torque, $\mathrm{m}_{\mathrm{SRM}}$, of an SRM moves the rotor counterclockwise to an applied clockwise current excitation sequence. This motion is shown in Fig. 20.
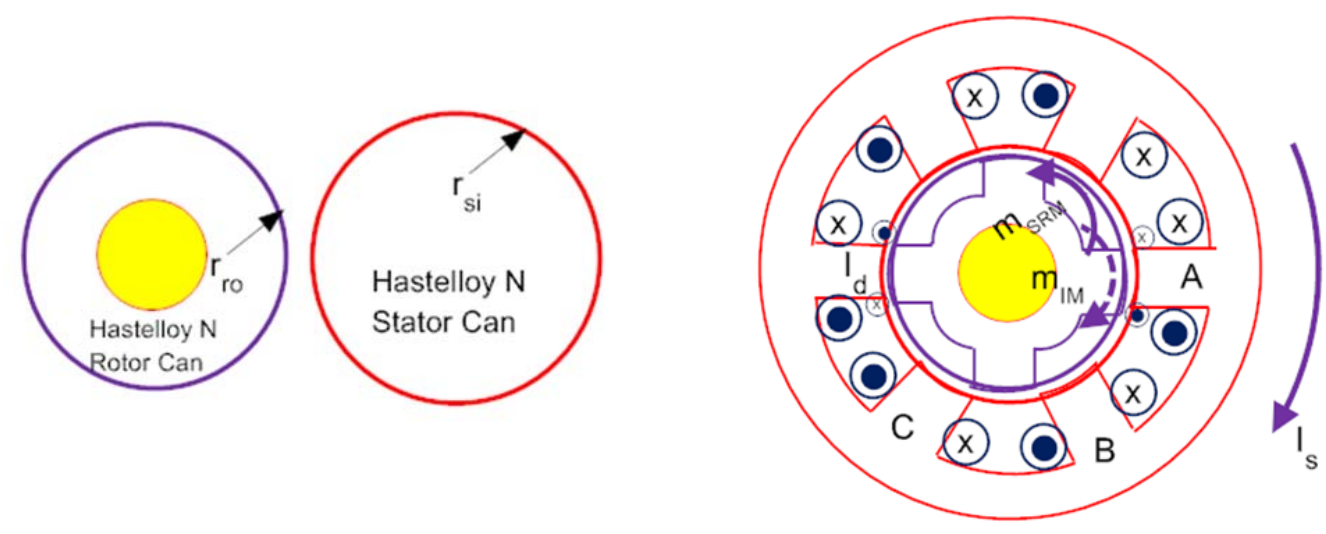

Fig. 20. Representative 6/4 SRM with rotor and stator conducting can and resultant torque components.

In conclusion, it is most beneficial in the development of this large-gap SRM to fully understand the implications of conducting shells surrounding both stator and rotor. For thin Hastelloy ${ }^{\circledR} \mathrm{N}$ cans, the inertial moment (IM) and damping effects are anticipated to be small, but then again, the large gap already acts to reduce the reluctance torque. A more detailed study of these effects is warranted.

\subsection{HEAT TRANSFER}

The standard equations for heat transfer are applicable for this system. The main sources of heat flux are convection and radiation from the exterior of the pump, joule heating of the motor and bearing stator coils, and heat transfer to the salt through conduction and convection.

To prevent overheating, the pump motor will include a cooling system that will take advantage of the reactor coolant being pumped and use it for motor cooling as well. To identify the necessary flow rates and operating pressures, the system has been modeled as a counterflow heat exchanger composed of two concentric cylinders circulating the fluid (Fig. 21). The outer cylinder represents the stator and stator can, which will be undergoing heat generation, and the inner cylinder represents the rotor can, rotor, and hollow shaft. The shaft will be considered stationary for the purposes of this model. 


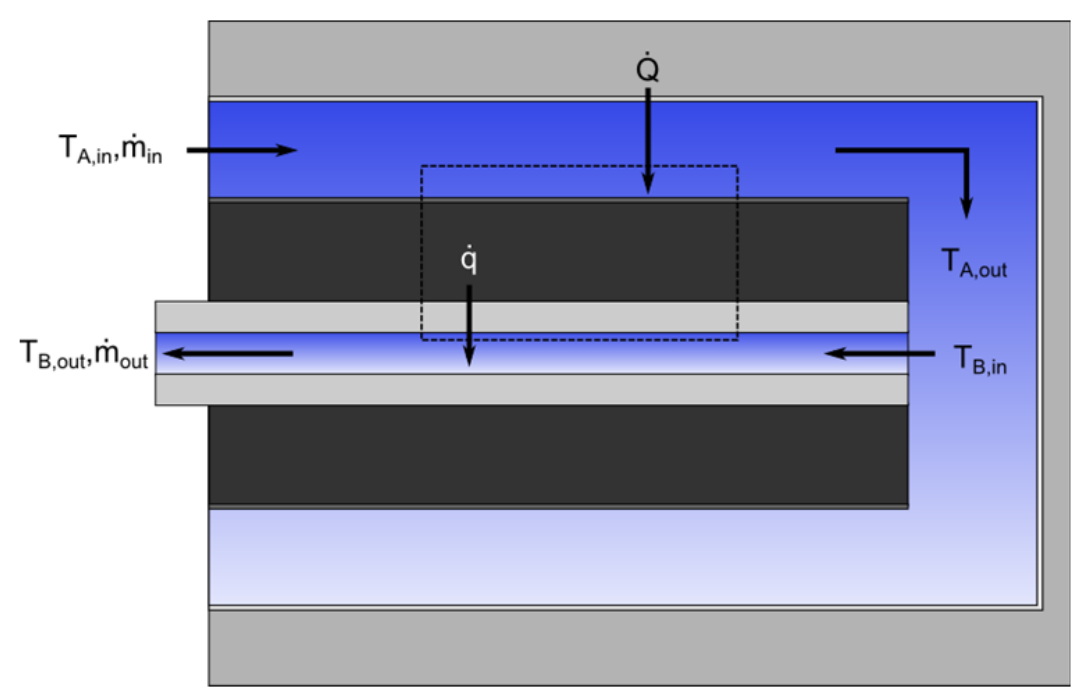

Fig. 21. Counterflow model orientation.

In this system, the fluid that flows around the annular region will also flow into the hollow shaft. Assuming $\mathrm{T}_{\mathrm{A}, \mathrm{out}}=\mathrm{T}_{\mathrm{B}, \mathrm{in}}$, then $\dot{\mathrm{q}}=0$, and

$$
\begin{gathered}
\dot{Q}=\dot{m}_{c} c_{p}\left(T_{\text {Ain }}-T_{\text {Aout }}\right) \\
\dot{m}=\frac{\dot{Q}}{c_{p}\left(T_{\text {Ain }}-T_{\text {Aout }}\right)}
\end{gathered}
$$

As the mass flow rate can be written as

$$
\dot{m}=\rho v A
$$

where $\rho$ is the fluid density, $\mathrm{v}$ is the velocity and $\mathrm{A}$ is the cross-sectional area, the inlet and outlet velocities can be expressed as

$$
v=\frac{\dot{m}}{\rho A}
$$

In order to estimate pressure drops across the pipes, the flow can be modeled as streamlines across a pipe and an annular region (Fig. 22). 


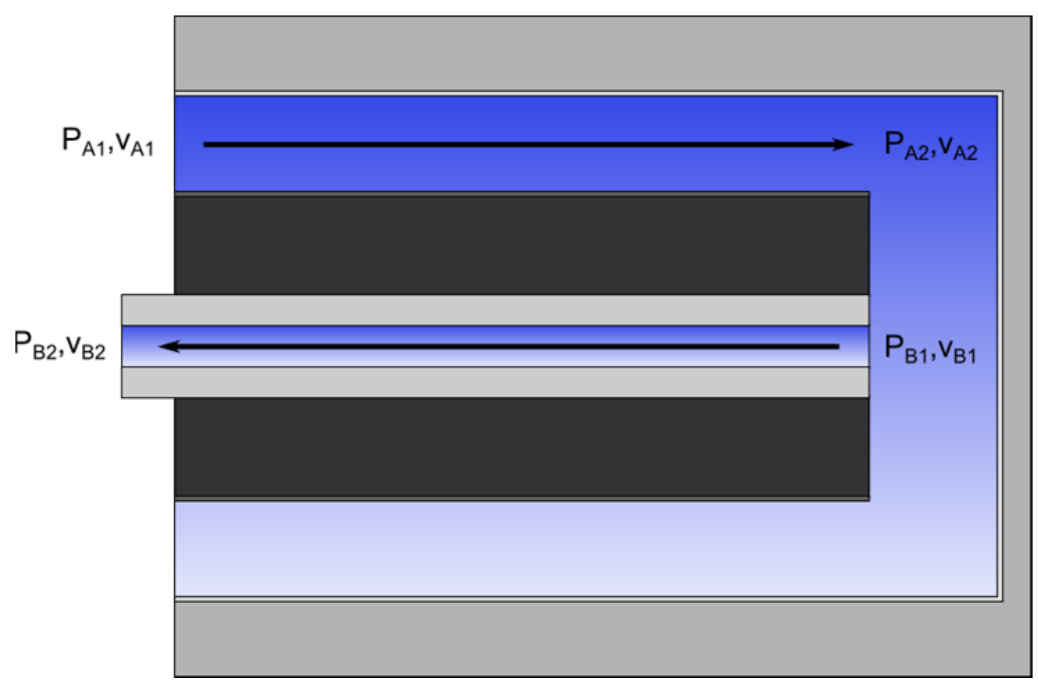

Fig. 22. Illustration showing streamlines in annular model.

For flow in a pipe,

$$
P_{1}+\frac{v_{1}^{2} \rho}{2}+z_{1} \rho g=P_{2}+\frac{v_{2}^{2} \rho}{2}+z_{2} \rho g+\frac{f L \rho v^{2}}{2 D}+\frac{K \rho v^{2}}{2}
$$

Given that the cross-sectional area along the inner pipe is constant, the velocities are the same, and assuming no height difference between the inlet and outlet, and no minor losses, the pressure drop can be expressed as

$$
P_{1}-P_{2}=\Delta P=\frac{f L \rho v^{2}}{2 D}
$$

where the friction factor $f$ is

$$
f=\frac{64}{R e}
$$

for fully developed laminar flow, and

$$
f=\frac{1}{(0.79 \ln R e-1.64)^{2}}
$$

for turbulent flow.

For the annular region, derivation from the Navier-Stokes equations in cylindrical coordinates yields 


$$
\Delta P=\frac{8 \mu L Q}{\pi}\left[r_{o}^{2}-r_{i}^{2}-\frac{\left(r_{o}^{2}-r_{i}^{2}\right)^{2}}{\ln \left(\frac{r_{0}}{r_{i}}\right)}\right]^{-1}
$$

where $\mathrm{Q}$ is the volumetric flow rate

$$
Q=v A
$$

The pressure drop between the gap between rotor and stator can be modeled as a $90^{\circ}$ elbow followed by a sharp-edge entrance with the cross sectional area of the inner pipe (Fig. 23).

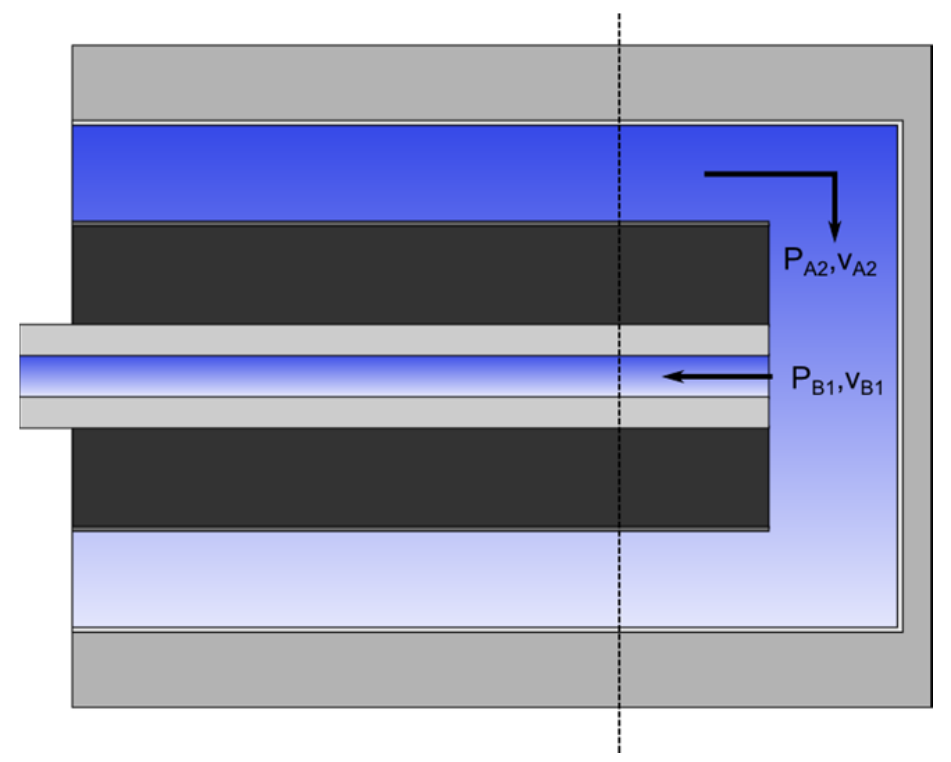

Fig. 23. Pressure drops in annular model.

The pressure drop follows from Equation (23):

$$
P_{B 1}-P_{A 2}=\frac{\rho}{2}\left(v_{A 2}^{2}-v_{B 1}^{2}\right)-K_{\text {elbow }} v_{A 2}^{2}-K_{\text {entrance }} v_{B 1}^{2}
$$

To estimate the pressure drop between the inner pipe and the annular region, the pressure drops across the system are taken into account, and

$$
\Delta P_{B 2 A 1}=\Delta P_{B 2 B 1}+\Delta P_{B 1 A 2}+\Delta P_{A 2 A 1}
$$

\subsection{MATERIALS}

Materials technology has evolved over the past four decades to the point where there are engineering materials that can withstand high temperatures, high stress levels, and the corrosiveness of fluoride salts associated with pumps for FHRs. Liquid fluoride salts act as fluxes and remove from the surface of the structural material any oxide layer, which normally provides for corrosion resistance at high temperatures. Thus, the approach normally used for providing high-temperature oxidation resistance, the development 
of an alloy composition that provides for an extremely slow growing protective oxide layer, typically chromium oxide and/or aluminum oxide, on the surface of the material, cannot be used.

The choice of a structural material for containment of liquid fluoride salts begins with a consideration of the reduction-oxidation (redox) potentials of the component elements of the material with respect to components of the salt at the temperature of interest. The desire is to have the components of the liquid fluoride salt be more stable than fluoride compounds that could form with the components of the structural material, and so have a non-reactive system. At the temperature of interest for this demonstration, nickle-based alloys with the appropriate alloying additions that allow for achieving the needed mechanical properties are the materials of choice. Corrosion performance can also be improved by the use of appropriate redox control strategies for the fluoride salts.

Corrosion performance of the materials of construction cannot be divorced from the environment in which the material is employed. Hence, while the redox considerations are of primary importance, impurities in the liquid fluoride salt contribute significantly to materials performance. Thus, the salt, FLiNaK, will undergo a rigorous cleaning process to remove moisture, oxides, sulfides, and other impurities, and processes will be implemented to prevent and/or ameliorate the ingress of air and moisture into the salt.

The $700^{\circ} \mathrm{C}$ operating temperature of this demonstration poses challenges for prolonged operation of materials. Diffusional processes will result in changes in the microstructures of the structural, electrical, and magnetic materials. These changes will affect temperature and time-dependent processes such as creep and fatigue of structural materials, and to a lesser extent, the electrical and magnetic properties of associated functional materials. Hence, time-temperature performance of the materials is important to the lifetime of the system.

Other materials issues that must be addressed are associated with the effects of high temperature on the microstructure of magnetic materials that may affect performance over time. The issues are (1) hightemperature insulation between magnetic materials; (2) high-temperature insulation for fine wires for inductive coils; (3) high-temperature insulation for wire-wound layers from each other; (4) a multitude of potentially associated mismatches in coefficients of thermal expansions of the various materials; and (5) trade-offs between materials and performance for items such as touch-down bearings.

\section{SYSTEM REQUIREMENTS AND DESIGN BASIS}

Defining system requirements is a necessary step leading to a conceptual design. In developing the system requirements, the mission requirements (performance, reliability, and fault tolerance), design strategies, and theoretical approaches are collectively considered to develop the system requirements.

At the outset of the project, it was determined that a reactor coolant circulating pump would be the component that would be redesigned to include embedded sensors and controls. The universal requirement of a coolant pump for advanced reactors is that it operates at high temperatures and represents a design that has no rotating seals. In addition, because of the close proximity of ORNL liquid salt loop, it was determined that the focus of the pump design be for a liquid salt application. As described in the Background (Section 2.4), a demonstration on the loop is planned for later in the project. 
Out these initial assumptions, a starting point was identified as follows.

- The motor-pump combination should be on a horizontal axis because the horizontal design would be more generally applicable to all other reactor designs.

- The system should be immersed in liquid salt at full operating temperature (eventually demonstrated on the salt loop).

- The pump should be a centrifugal type, which has relatively high conversion efficiency.

- The motor should be a canned rotor design with no rotating seals, allowing full isolation between reactor cooling and the outside (containment) environment.

- Penetrations through the barrier can should be minimized (target is zero penetrations).

- The rotating shaft should be fully magnetically suspended under all operating conditions.

- The motor should have non-magnetic catcher bearings for holding the rotor when not operating.

\subsection{CONTROL SYSTEMS}

The control system will be divided into the control system for the active magnetic bearing and the control system for the switched reluctance motor. The most basic requirement for the bearing and motor control systems is stability. However, in addition to stability, there are a number of performance requirements, requirements arising from the system geometry, and robustness requirements that need to be fulfilled. These will be detailed in Sections 5.1.1 and 5.1.2. We will only consider the radial active magnetic bearings. The axial magnetic bearings are treated in a similar manner.

\subsubsection{Active Magnetic Bearings}

The most critical design requirement of the active magnetic bearings control system for normal operation is the boundedness of the system states to the bounded disturbances arising from the impeller forces, motor forces, gravity, and external forces on the pump. The bearing disturbances $w(t)$ enter into the system as shown in the block diagram in Fig. 24.

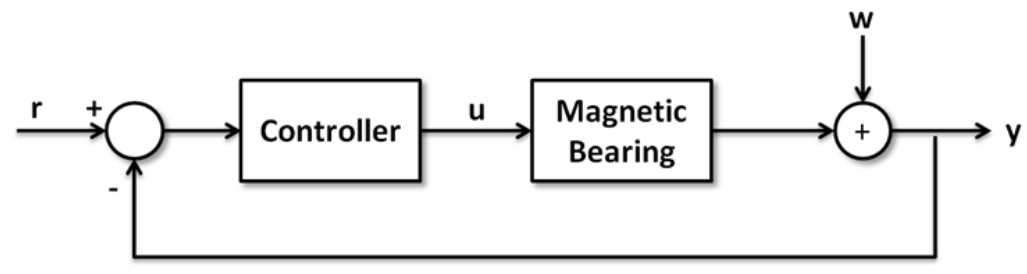

Fig. 24. Disturbance injection into the control system.

The impeller disturbance forces arise from the finite number of blades causing pulses of torque off centered from the rotor axis. The frequency of the disturbance is $f_{\text {dist }}=n \omega$, where $n$ is the number of rotor blades and $\omega$ is the rotational velocity of the rotor. Gravity provides constant disturbance force, external vibrations are a time-varying disturbance force, and the largest external disturbance to account for is earthquake-induced vibrations. The correlation between the rotor position and the bearing and motor magnetic gaps indicates that bearing disturbances affect the motor controller and vice versa. If the rotor moves from geometric center, the variations in the magnetic-gap around the circumference of the motor will cause uneven coil forces, which in turn will cause an axial force disturbance on the bearings.

Under the influence of these disturbance forces, the rotor cannot move more than the allowable gap or the rotor will crash, causing damage to the system and necessitating maintenance. This constraint can be thought of as designing a virtual spring into the controller that is stiff enough to bound the movement of 
the bearing rotor under the maximum expected load. Let $F_{\max }$ be the maximum force on the canned rotor and let $d_{\max }$ be the maximum allowable displacement of the rotor. This implies that the control system must have an effective spring rate of

$$
k_{e f f}>\frac{F_{\max }}{d_{\max }}
$$

It can be seen from equation (15) that the effective spring rate of the system is given by

$$
k_{\text {eff }}=B\left(K_{s}+K_{i} K_{p}\right) C
$$

and the effective damping coefficient is

$$
d_{\text {eff }}=G+B K_{i} K_{d} C \text {. }
$$

For example, a system with a fluid gap between the rotor and the stator can of $1.0 \mathrm{~mm}$ and touch down bearings that protrude beyond the stator can an additional $0.25 \mathrm{~mm}$ yields an effective gap size of $0.75 \mathrm{~mm}$. Setting the maximum rotor movement to $d_{\max }= \pm 0.6 \mathrm{~mm}$ and assuming a maximum disturbance force of $F_{\max }=200 N$ yields a minimum effective spring force for the system of 266.7 $\mathrm{N} / \mathrm{mm}$.

A more sophisticated method to ensure stability and performance in the presence of sensor noise, model uncertainty, and exogenous force inputs is robust control design theory. By implementing robust control, a more advanced control design technique than PID, guarantees can be made about system stability in the presence of these uncertainties. We begin by defining a vector of performance outputs $z$. These can include rotor displacements, output voltages, amplifier currents, and flux densities, to name a few potentials. Each of these performance measures has a bound based on the physical constraints of the system. The following is adapted from [40]. From the closed loop system equation, the transfer function from the exogenous inputs $w$ to the performance measures $z$ can be represented as Equation (34):

$$
z=G_{c l}(s, \omega) w
$$

The performance measures are described as $\left|z_{i}\right|<z_{\max , i}$, and the exogenous inputs are bounded as

$$
\left|w_{i}\right|<w_{\max , i}
$$

Each performance measure is normalized as follows:

$$
\bar{z}_{i}=\frac{1}{z_{\max , i}} z_{i} \Rightarrow \bar{z}=W_{z} z: W_{z}=\operatorname{diag}\left(1 / z_{\max , i}\right)
$$

and each exogenous input is normalized in a similar manner by

$$
\bar{w}_{i}=\frac{1}{w_{\max , i}} w_{i} \Rightarrow W_{w} \bar{w}=w: \quad W_{w}=\operatorname{diag}\left(w_{\max , i}\right)
$$


The transfer function from the normalized disturbance to the normalized performance measure is

$$
\bar{z}=W_{z} G_{c l} W_{w} \bar{w}
$$

For the system to be robust to the disturbances, we require that

$$
\left|z_{i}\right|<z_{\max , i}, \quad \forall\left|w_{i}\right|<w_{\max , i}
$$

This statement is equivalent in the normalized vectors to

$$
|\bar{z}|_{\infty}<1, \quad \forall|\bar{w}|_{\infty}<1
$$

This condition is computationally intractable due to the large combination of possible disturbance inputs, but equivalence has been developed that is mathematically tractable. The following is equivalent;

$$
|\bar{z}|_{\infty}<1, \quad \forall|\bar{w}|_{\infty}<1 \quad \Leftrightarrow \quad \bar{\sigma}\left(W_{z} G_{c l} W_{w}\right)<1
$$

\begin{tabular}{|c|c|c|}
\hline Variable & Bound & Description \\
\hline$x_{a}$ & $0.6 \mathrm{~mm}$ & Bearing A x-position \\
\hline$y_{a}$ & $0.6 \mathrm{~mm}$ & Bearing A y-position \\
\hline$x_{b}$ & $0.6 \mathrm{~mm}$ & Bearing B x-position \\
\hline$y_{b}$ & $0.6 \mathrm{~mm}$ & Bearing B y-position \\
\hline$i_{a x}$ & $20 \mathrm{~A}$ & Bearing A, amplifier current, $\mathrm{x}$-axis \\
\hline$i_{a y}$ & $20 \mathrm{~A}$ & Bearing A, amplifier current, $y$-axis \\
\hline$i_{b x}$ & $20 \mathrm{~A}$ & Bearing B, amplifier current, $\mathrm{x}$-axis \\
\hline$i_{b y}$ & $20 \mathrm{~A}$ & Bearing B, amplifier current, $y$-axis \\
\hline$\Phi_{a x}$ & $1.8 \mathrm{~T}$ & $\begin{array}{l}\text { Saturation flux in bearing A x-axis (due to material } \\
\text { properties) }\end{array}$ \\
\hline$\Phi_{a y}$ & $1.8 \mathrm{~T}$ & $\begin{array}{l}\text { Saturation flux in bearing A y-axis (due to material } \\
\text { properties) }\end{array}$ \\
\hline$\Phi_{b x}$ & $1.8 \mathrm{~T}$ & $\begin{array}{l}\text { Saturation flux in bearing } \mathrm{B} x \text {-axis (due to material } \\
\text { properties) }\end{array}$ \\
\hline$\Phi_{b y}$ & $1.8 \mathrm{~T}$ & $\begin{array}{l}\text { Saturation flux in bearing B y-axis (due to material } \\
\text { properties) }\end{array}$ \\
\hline
\end{tabular}

where $\bar{\sigma}(\bullet)$ is the maximum singular value.

In the case of the active magnetic bearings, the performance metrics and their limits are given in Table 7.

Table 7. Performance bounds for controller design

The exogenous disturbances and their bounds are given in Table 8. The nominal operating speed of the canned pump is designed at $60 \mathrm{~Hz}(3600 \mathrm{rpm})$. 
Table 8. Disturbance bounds

\begin{tabular}{lll}
\hline \multicolumn{1}{c}{ Description } & \multicolumn{1}{c}{ Bound } & \multicolumn{1}{c}{ Frequency } \\
\hline Impeller force & $30 \mathrm{~N}$ & $0-120 \mathrm{~Hz}$ \\
Rotor imbalance & $10 \mathrm{~g}-\mathrm{mm}$ & $0-60 \mathrm{~Hz}$ \\
Earthquake & $0.5 \mathrm{~m} / \mathrm{s}^{2}$ & $0.8 \mathrm{~Hz}$ \\
Motor & $45 \mathrm{~N}$ & $360 \mathrm{~Hz}$ \\
External vibrations & $0.0001 \mathrm{~m} / \mathrm{s}^{2}$ & $0-2000 \mathrm{~Hz}$ \\
\hline
\end{tabular}

Similarly, the maximum acceptable sensor noise that the controller can tolerate and remain stable can be determined for the system using the small gain theorem. ${ }^{41}$

Another important requirement on the control system is the maximum allowable controller bandwidth. The bandwidth of the closed loop system is defined as the frequency where the output drops $-3 \mathrm{~dB}$ below the zero frequency value. The maximum bandwidth is set by a number of factors. The primary limitation on controller bandwidth is the bandwidth of the discrete system, both sensor and power electronics. The closed loop system must have a bandwidth of less than half the bandwidth of the discrete electronics. There are also bandwidth limitations due to the eddy currents that form in the stator and rotor cans. The eddy currents also insert a time delay into the control action which must be accounted for along with the delays inherent in the power electronics.

Consider the required bandwidth from the canned motor sensed parameter shown at left in Fig. 7 through the cascade of input electronics (signal conditioning of transducer output) through the signal processing, control system block, and drive electronics to the motor stator. Consider the current regulation function of the SRM stator excitation currents. For robust performance in normal operation, the bandwidth of this cascade should be about $3 \mathrm{kHz}$. Modern digital controls (late developments in Fig. 3) based on high-speed FPGA technology are capable of realizing current regulation bandwidth up to one-third the switching frequency of the power inverter, but more realistically, at half that. Nominal switching frequency of the power inverter is $f_{s}=10 \mathrm{kHz}$, so a $3-\mathrm{kHz}$ bandwidth is feasible.

The current regulator is an inner loop function and therefore must have high bandwidth. The test platform speed control bandwidth can be $700 \mathrm{~Hz}$ for a FPGA or digital signal processing implementation of digital controller. This specification would fit a servo control motor. However, for this pump application there is no pressing need for speed control regulation higher than perhaps $300 \mathrm{~Hz}$ at most. Therefore, based on this information, the following performance specification applies.

- Switching frequency, $f_{s} \sim 10 \mathrm{kHz}$ using commercially available IGBTs

- Current regulator bandwidth of $1.5 \mathrm{kHz}$ to $3 \mathrm{kHz}$

- Current sensor bandwidth of $50 \mathrm{kHz}$

- Speed control bandwidth of $150 \mathrm{~Hz}$ to $300 \mathrm{~Hz}$

- Encoder, or sensorless position control resolution of 12 to 14 bits

- Nominal zero-order hold ( $\mathrm{ZOH})$ execution of inverter PWM introduces less than $1.5 t_{s}$ delay

For example, a 12 bit resolution encoder is accurate to $0.088^{\circ}$ mechanical, sufficient for the $6 / 4$ SRM canned motor. Higher resolution can be obtained from a resolver, and this is a candidate for highperformance torque and speed control. With a maximum operating speed of $3600 \mathrm{rpm}$, a 12 to 14 bit encoder is sufficient. 
The system bandwidth for the cascade of five-signal conditioning, control block, drive electronics, and the power inverter-motor plant of Fig. 11 depends on whether the individual bandwidths are identical (or less than an octave apart), or if one has the dominant pole. Suppose the five components shown horizontally in Fig. 8 are each $f_{H}=10 \mathrm{kHz}$, where $f_{H}$ is the high-frequency corner point. Then the system bandwidth for $n=5$ stages is $f_{H}{ }^{*}=3.86 \mathrm{kHz}$ according to Equation (41). If the nearest pole relative to a dominant pole is more than two octaves apart, then the system bandwidth is within 94 percent of the dominant pole bandwidth. System engineering of the pump motor control signal transducer to actuator (the motor) consisting of a cascade of several signal processing stages therefore requires each individual stage to have higher bandwidth than the total required. Typical motor drive current sensors are nominally $50 \mathrm{kHz}$ bandwidth because of this effect.

$$
f_{H}^{*}=\sqrt{\left(2^{\frac{1}{n}}-1\right)} f_{H} .
$$

\subsubsection{Switched Reluctance Motor}

There are multiple sources of disturbances affecting the motor operation. The pump impeller is the most constant disturbance source. The impeller will also cause a disturbance in the bearing position that will in turn affect the motor operation due to the change in the motor magnetic gap. The rotational velocity of the controller firing sequence should not be affected by the changes in magnetic gap; however, to maintain sufficient torque for operation, the magnitude of the coil currents needs to be increased where the gap is larger and decreased where it is smaller to maintain an even torque profile.

There is a wide variety of techniques used for SRM control. Basic control methods utilize turn-on and turn-off (or duty) switching angles with respect to rotor position. These angles vary both with speed and torque level, and current limiting is often implemented to prevent overheating or over-current conditions for the converter. As speed increases with a given torque level, the turn-on angle must be increased in order to overcome the increasing reactance, which is proportional to electrical frequency. Therefore, even though the SRM does not have permanent magnets, the model effectively contains a back- electromotive force (EMF) component. If high levels of power are needed for a brief amount of time, the SRM can be operated in what is called "continuous conduction mode." In this approach, the phase current never reaches zero, and although generative torque is briefly incurred, the overall torque and power output are substantially higher. The amount of energy converted to mechanical energy is known as co-energy and is labeled as $\mathrm{W}_{\mathrm{f}}$ in Fig. 25. As speed increases, the capability of developing current in the winding decreases. and therefore, the co-energy loop becomes smaller. By allowing the minimum current to be non-zero, higher current levels can be achieved and more electrical energy is converted to mechanical energy. Efficiency suffers and torque ripple increases during this operation condition and therefore is not appropriate for long duration. 

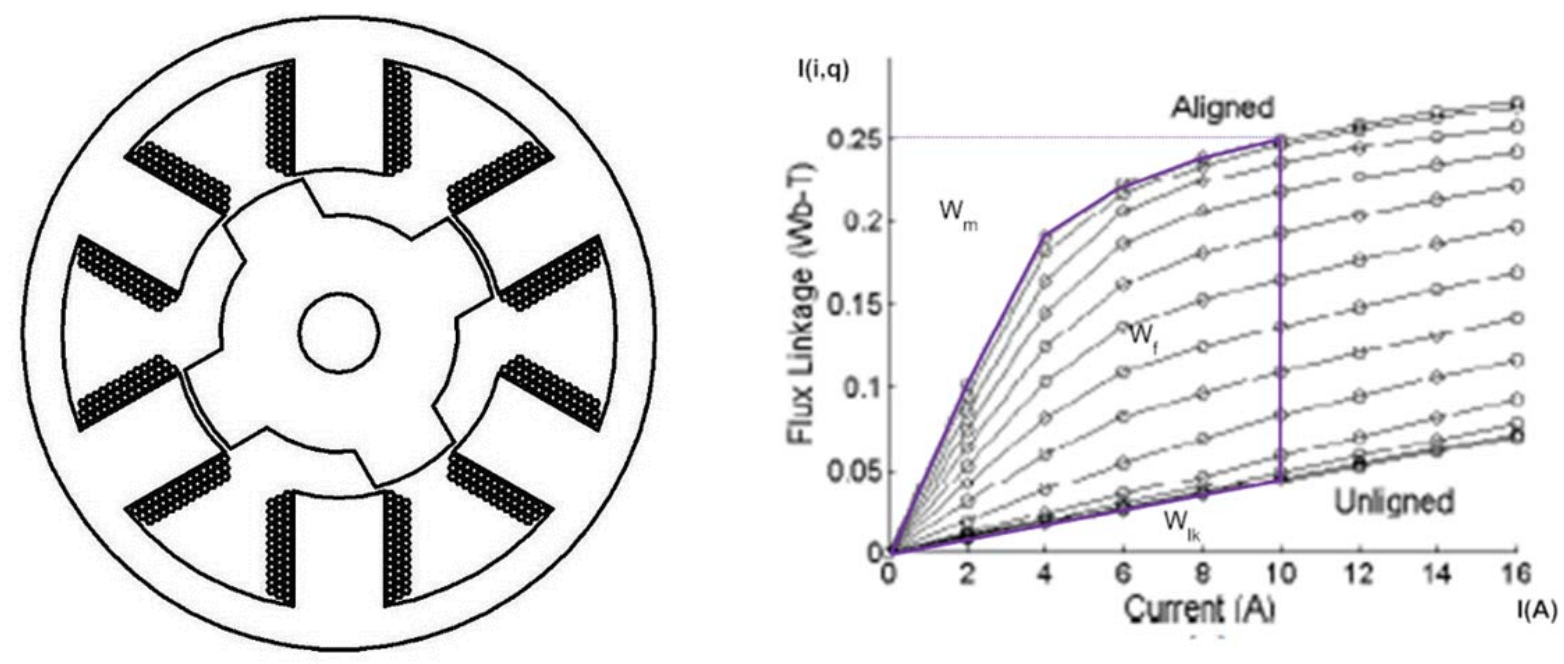

Fig. 25. SRM and flux linkage diagram.

The converter shown in Fig. 26 has three phases, with two IGBTs and two Diodes in each phase. Both upper and lower IGBTs must be on to apply voltage (+V DC) to the windings. If both IGBTs are off, no voltage is applied, but if the IGBTs were just turned and current is still flowing, the free-wheeling diodes are active, and -V DC is effectively applied to the windings. Current regulation using these two conditions is known as "hard chopping." Another option is to keep one IGBT on and turn the other IGBT off when a slight reduction in current is desired. No voltage is applied to the winding in this case (known as the "zero volt loop"), allowing the current to decrease due to the winding resistance and semiconductor losses. Use of this switching state is known as "soft chopping," and it can yield reductions in acoustic noise and torque ripple. With increasing current, soft chopping voltages can switch from $0 \mathrm{~V}$ to $+\mathrm{V} \mathrm{DC}$, and with decreasing current the voltages switch from $0 \mathrm{~V}$ to $-\mathrm{V} \mathrm{DC}$, with the latter condition achieved with both IGBTs in the off condition and the free-wheeling diodes forward biased as long as current is greater than zero. For soft chopping, either IGBT can be left on to create the zero volt loop, but there is a PWM switching technique that alternates IGBTs for the zero volt loop each switching cycle. This interleaved switching approach allows for even thermal conditions amongst the switches and diodes and the effective switching frequency across the windings doubles, which is a significant advantage for current regulation and acoustic noise reduction. 


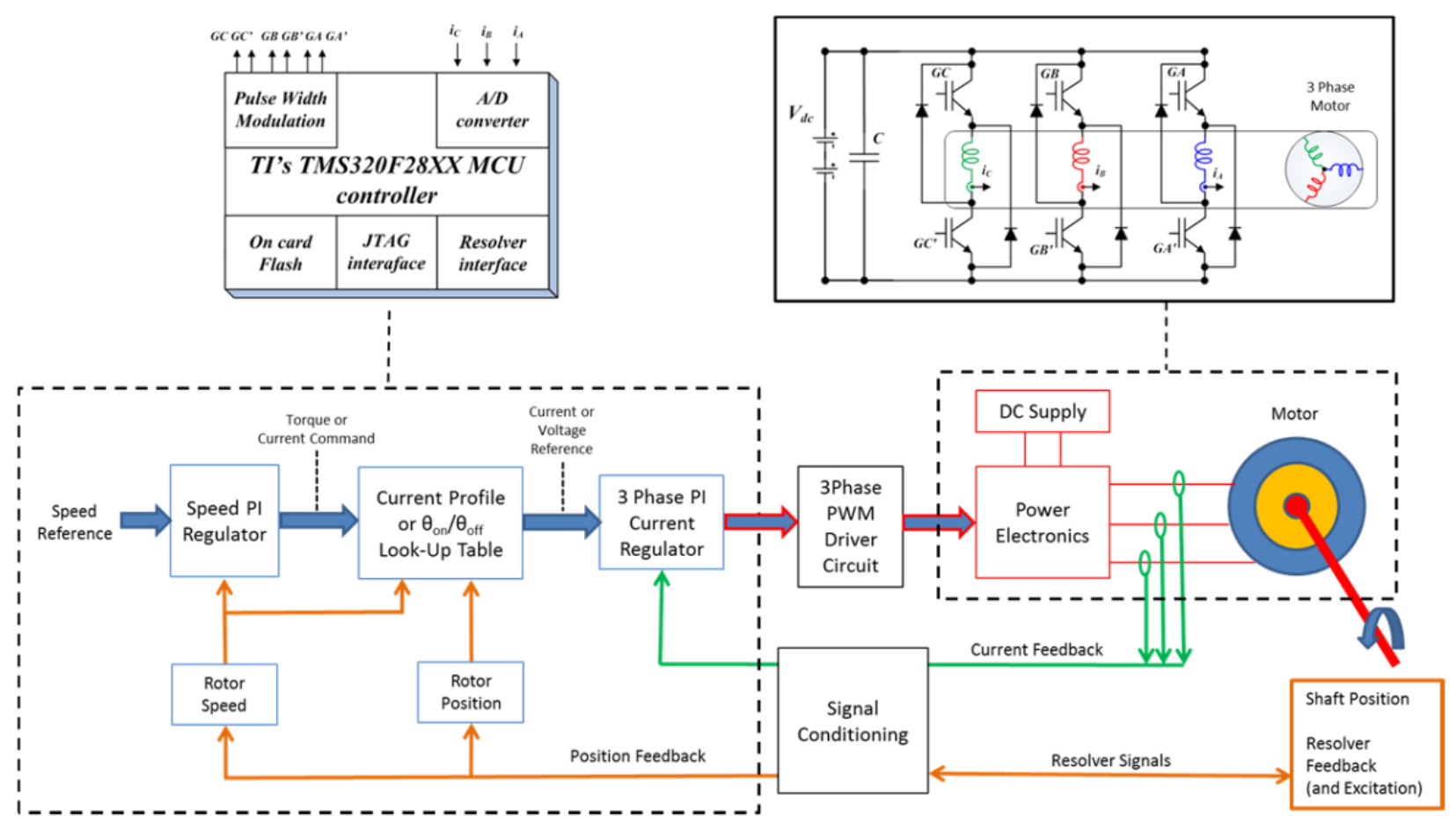

Fig. 26. Switched reluctance motor speed control block diagram.

The switching states mentioned above are possible regardless of the type of control technique that is implemented. Sophisticated control techniques involve the use of current profiling to achieve higher operational efficiency and/or reduced torque ripple and acoustic noise. This approach often requires sophisticated observer-based controls, where the machine is modeled within the controller or lookup tables provide information necessary to determine the optimal profile. Flux linkage observers are often used as a primary control mechanism since this quantity directly relates to energy conversion and it is sometimes easier to regulate than current, since converters are voltage driven. Additionally, this type of sophistication will be required for redundant sensorless position feedback from the SRM, and therefore may be well suited for this application.

\subsection{MOTOR/PUMP SYSTEMS}

\subsubsection{Pumping Characteristics}

The pump consists of a horizontal shaft, single-stage, centrifugal, high-temperature, liquid pump that is compatible with liquid fluoride salts. The pump chosen for this system will have the characteristics of the custom-made vertical pump designed for the experimental salt-loop system located at ORNL. Pump dimensions and important parameters are given below in Table 9. 
Table 9. Pump and system characteristics

\begin{tabular}{lrl}
\hline & \multicolumn{1}{c}{ Pump data } & \\
Impeller disk radius & & $14.29 \mathrm{~cm}$ \\
Inlet diameter & $5.08 \mathrm{~cm}$ \\
Outlet diameter & $6.35 \mathrm{~cm}$ \\
Corresponding pipe size & TBD \\
Shaft mass & $5.05 \mathrm{~kg}$ \\
Shaft unbalance & $0.01 \mathrm{percent}$ \\
& & \\
Rotational speed & $60 \mathrm{~Hz}$ \\
& & \\
Cover pressure & System parameters & $275.8 \mathrm{kPa}$ \\
Temperature & $600-750{ }^{\circ} \mathrm{C}$ \\
Static head & $1.68 \mathrm{~m}$ \\
Static pressure & $33 \mathrm{kPa}$ \\
Suction head & $-0.37 \mathrm{~m}$ \\
& \\
Flow rate & Fluid characteristics & $4.5 \mathrm{~kg} / \mathrm{s}$ \\
Density & $2019 \mathrm{~kg} / \mathrm{m}^{3}$ \\
Inlet velocity & $0.27 \mathrm{~m} / \mathrm{s}$ \\
Outlet velocity & $0.18 \mathrm{~m} / \mathrm{s}$ \\
\hline
\end{tabular}

\subsubsection{Duty Cycle Profiles}

Reactor cooling water or cooling salt pumps are intended to operate at 100 percent rated flow and speed for typical operation. This typical operation may have durations of 18 months between refueling events. Exceptions to this would include cooling startup after a refueling event, cooling shutdown to begin a refueling event, or other very infrequent reactions to a failure event. The target life and duty cycle is 7 years at 100 percent pump rated speed.

Reactor coolant-pump overspeed condition can be postulated for either a fault in the connected electrical system that results in an increase in the frequency of the supplied current or due to a pipe rupture that results in an increase in the flow through the pump as the coolant exits the pipe. ${ }^{42}$ The control system should limit the overspeed to 110 percent for short durations.

For grid-disconnect transients or turbine trips actuated by either the reactor trip system or the turbine protection system, the turbine overspeed control system acts to limit the reactor coolant-pump overspeed. The turbine control system acts to rapidly close the turbine governor and intercept valves.

An electrical fault requiring immediate generator trip (with resulting turbine trip) will result in an overspeed condition in the electrically coupled reactor coolant pump no greater than that described previously for the grid disconnect/turbine trip transient.

Pump overspeed from high coolant flow rates associated with pipe rupture events are mitigated by the inertia of the pump, flywheel, and motor and by the connection of the motor to the electrical grid. Because of the application of mechanistic pipe break criteria, dynamic effects such as pump overspeed are not evaluated for breaks in piping in which leak-before-break is demonstrated. 
To satisfy post-maintenance testing, control system commissioning, and other infrequent activities, the pump system must be capable of operation down to $3 \mathrm{~Hz}$ ( 5 percent of rated speed) with reasonable control stability and speed regulation.

\subsubsection{Steady State and Transient Hydraulic Response}

\section{Steady State}

The pump will provide no greater than a $\pm 2 \%$ steady-state speed following error for $80-110 \%$ of rated speed and rated flow (5.2.1). The speed following error is defined as the difference between the desired pump speed and the actual measured speed while the pump-speed desired command is at steady state (has not changed for a reasonable duration).

Transient (Slew Rate, Rise Time, Tracking Error, Settling Time)

1. The pump should be capable of a speed slew rate (rate of change) up to $60 \mathrm{rpm} / \mathrm{sec}$.

2. The pump speed command will utilize a rate limit of $10 \mathrm{rpm} / \mathrm{sec}$ during normal operation.

3. The pump control will be capable of responding to a $\pm 10 \%$ step variation in load by returning the pump to the desired pump speed command not to exceed a 2 second rise time, not to exceed 10 percent overshoot, and not to exceed a settling time of 4 seconds.

The rise time is defined as the time required for the step response to rise from 10 to 90 percent of the final value. The percent overshoot is the difference between the pre-step response steady-state value and maximum step response value divided by the difference between the pre- and post-step response steadystate values. The settling time is defined as the time for the step response to achieve steady state.

\subsubsection{Motor Thermal Management}

The extreme temperatures involved in the molten salt system require materials to operate near to the limit of their mechanical and electrical properties. This means that the thermal management system needs to be carefully analyzed and designed to maintain the system within the safe operating range. The five systems that have specific temperature requirements for the different operating states are the

1. external surface,

2. stator windings,

3. stator can,

4. molten salt, and

5. rotor

The external surface temperature needs to remain as low as possible during operation for personnel safety while the temperature of the salt needs to be maintained at the nominal operating temperature of $700^{\circ} \mathrm{C}$ to prevent solidification and maintain efficient system operation. Additionally, temperature limitations on the stator winding insulation require a temperature below $500^{\circ} \mathrm{C}$ to remain with the manufacturer's recommended temperature. Discussions with wire vendors have led to wire insulation possibilities that might work at the nominal operating temperature, but the consequences of insulation failure are high; therefore, the thermal management system will be designed to keep the coil temperatures below $500^{\circ} \mathrm{C}$.

The main sources of heat in the system are the motor windings, the bearing windings, and the salt. From Section 5.5.1, the initial estimate shows that each coil has 24 turns/coil with 12 strands of 20 American 
Wire Gauge (AWG) wire per turn. Assuming a tooth cross-sectional dimension of $60 \mathrm{~mm}$ by 34.817, $\mathrm{mm}$ the circumferential length of a turn is $\mathrm{L}=229.38 \mathrm{~mm} / \mathrm{turn}$. At room temperature, the resistance of 20

AWG wire is

$3.331 \times 10^{-5} \Omega / \mathrm{mm}$. Using the equation for temperature-dependent resistance,

$$
R(T)=R\left(T_{0}\right)(1+\alpha \Delta T)
$$

at $700^{\circ} \mathrm{C}$ the resistance of $20 \mathrm{AWG}$ wire becomes $1.2126 \times 10^{-4} \Omega / \mathrm{mm}$. This yields a resistance per strand of $0.6675 \Omega$. With 12 wires in parallel and a 200 A current, the total winding resistance is $0.0556 \Omega$ and the power dissipated per coil is $2225 \mathrm{~W}$.

To prevent overheating, the pump motor will include a cooling system, which will take advantage of the reactor coolant being pumped, and use it for motor cooling as well. To identify the necessary flow rates and operating pressures, the system has been modeled as a counter-flow heat exchanger composed of two concentric cylinders circulating the fluid. Estimated mass flow rates and resulting pressure drops between the inner and outer cylinders, for a range of heat transfer rates from the stator, are given below, assuming an allowable temperature rise of $5^{\circ} \mathrm{C}$. Mass flow rate and pressure drop between fluid gap and shaft inner cylinder as a function of stator heat generation is shown in Figs. 27 and 28.

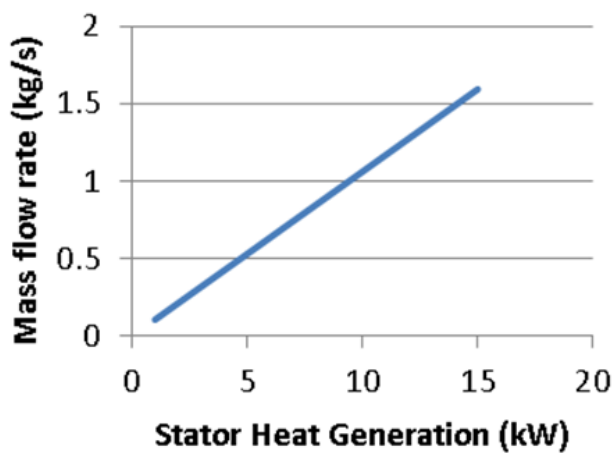

Fig. 27. Mass flow in gap.

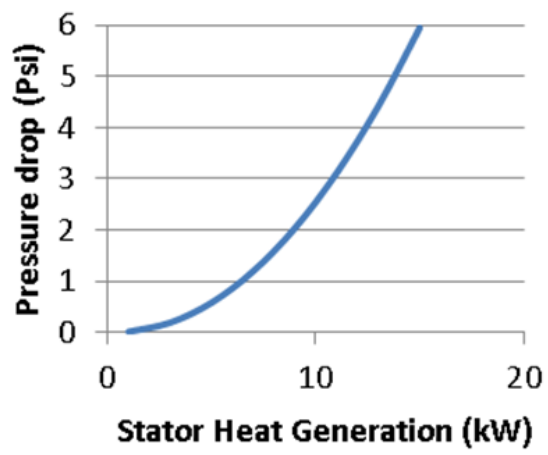

Fig. 28. Pressure drop through gap.

For pumps with a higher torque rating, additional cooling may be required to dissipate the heat generation in the coils. Care must be taken to guarantee that the inner wall of the stator can does not drop below the freezing point of the salt.

\subsection{MOTOR MECHANICAL PERFORMANCE}

\subsubsection{Single Stage, Centrifugal, Horizontal Shaft}

The chosen pump system is the single-stage centrifugal, horizontal pump. In this system, fluid salt enters through the center of the pump and is redirected outwards through the outlet by a single impeller contained within a volute. A diagram of the cross section of the pump is given below in Fig. 29. For more information on the mechanical design, see Appendix A. 


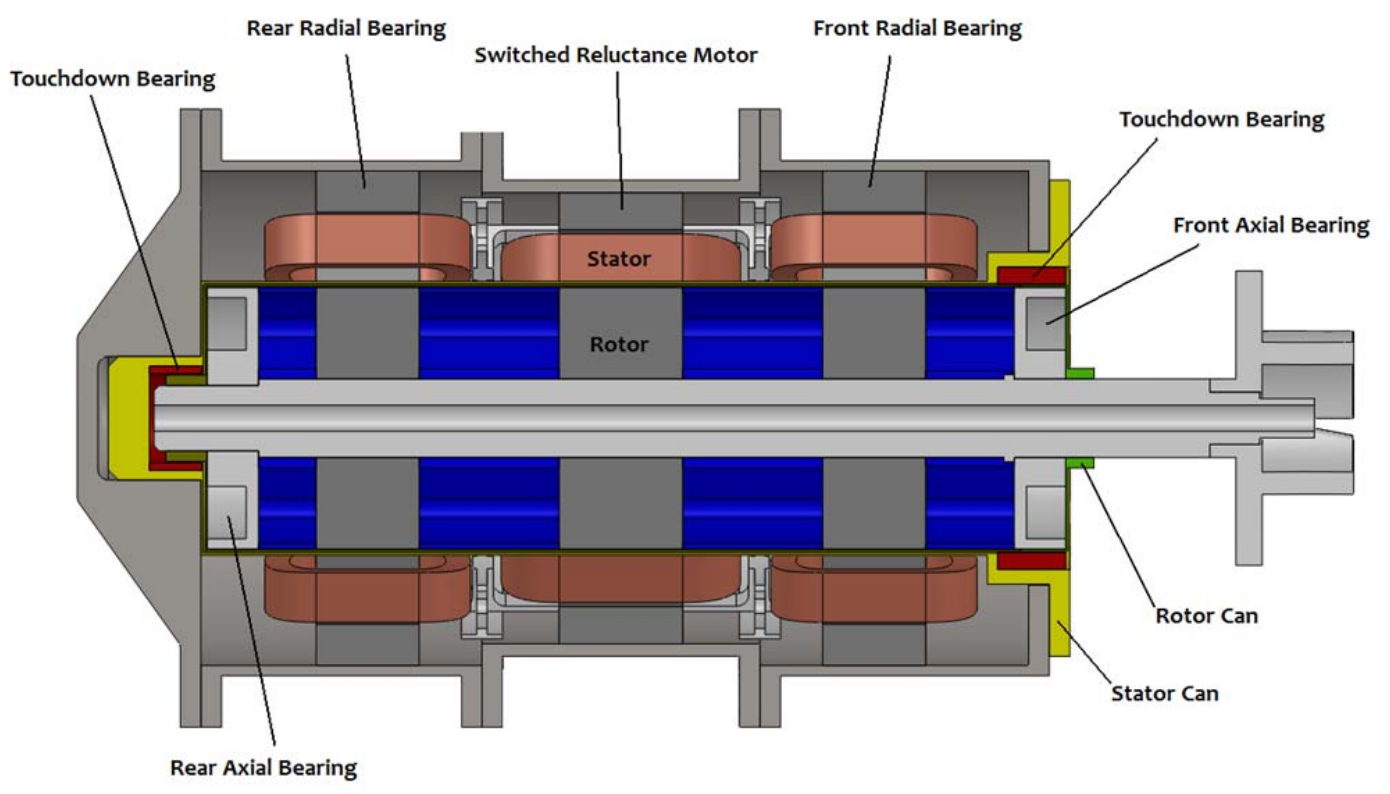

Fig. 29. Motor bearing and associated component location.

\subsubsection{Catcher Bearing Size, Geometry, Gap}

As shown in Fig. 29, catcher bearings (touch-down bearings) will be placed at the front and back end of the shaft. The front catcher bearings will be between the stator can and the rotor can; hence, it will be exposed to the molten salt. The back bearing will be between the stator can and the axle. Silicon carbide has been chosen for the bearing material.

\subsubsection{Catcher Bearing Forces}

Failure of active magnetic bearings and subsequent rotor contact with touch-down bearings can result in different types of dynamic behavior. Possible states of motion are a reverse or backward whirl, chaotic jumping of the rotor, oscillations at the base of the catcher bearing, or a forward whirl. These states of motion are illustrated in Fig. 30.

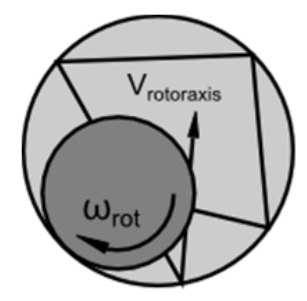

Jump

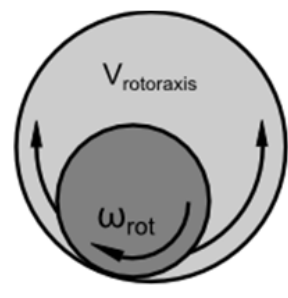

Oscillation

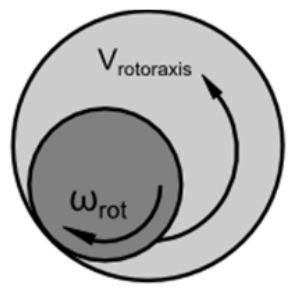

Forward

Whirl

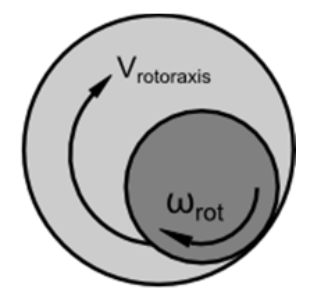

Backward

Whirl

Fig. 30. Bearing dynamic motion categories (adapted from ${ }^{[40]}$ ).

In the case of a bearing malfunction in which the rotor can falls on the catcher bearings, the catcher bearings must be able to withstand the forces and moments of the fall. A free body diagram of the rotor is given in Fig. 31. 


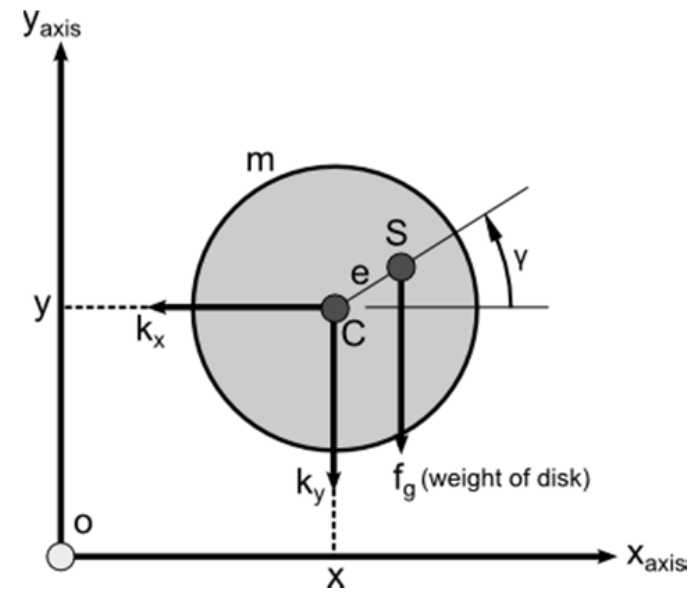

Fig. 31. Bearing free-body diagram (adapted from Ref. 34).

Assuming the motor is not rotating, the catcher bearings must be able to withstand the stationary weight of the rotor and impeller. Making the simplifying assumption of even mass distribution, we may estimate the forces that will be exerted on each bearing in the case of a collision (Fig. 32). For expected values of these forces, refer to Appendix A, Sect. A.2.1.

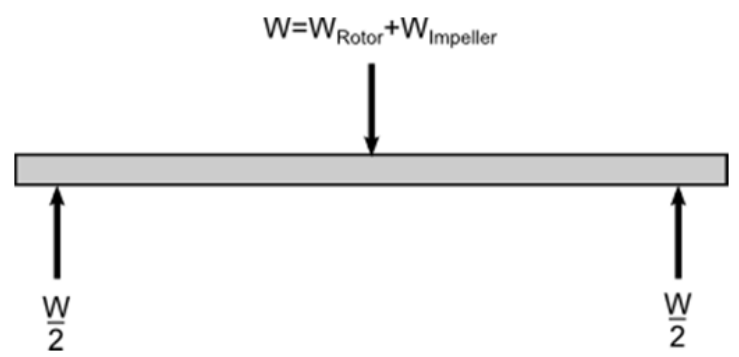

Fig. 32. Static force balance (adapted from Ref. 34).

While impact physics is complex, the collision between the rotor and the catcher bearings can be initially modeled as a nonlinear impact vibration model consisting of a spring-damper system, as shown in Fig. 33.

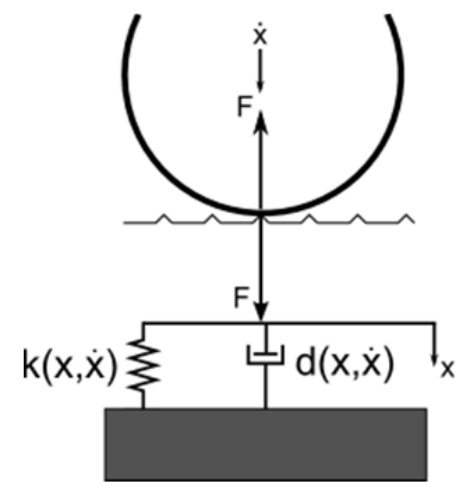

Fig. 33. Impact vibration model (adapted from Ref. 34).

The normal force $\mathrm{F}$ will be equivalent to the force $\mathrm{W} / 2$ as shown in the free body diagram above. Equations of motion for the system can be found in terms of the spring and damping constants. 


$$
-F=m \ddot{x},
$$

where $F=F_{D}+F_{E}$ and $F_{E}$ and $F_{D}$ are the elastic and damping forces, respectively. Boundary conditions are given below:

$$
\begin{aligned}
& x(0)=0 \\
& \dot{x}(0)=v_{i},
\end{aligned}
$$

and the elastic force can be approximated by Hertzian Contact:

$$
F_{E}=k x^{n}
$$

where $n$ is between 1 and $\frac{3}{2}$.

The damping force can be described as

$$
F_{D}=\lambda x^{n} \dot{x}
$$

where the constant $\lambda$ can be derived as

$$
\lambda=\frac{3}{2} \alpha k
$$

and $\alpha$ is related to the impact restitution coefficient, which depends on the two materials that are making contact.

The equations of motion of the system can then be expressed as

$$
m \ddot{x}=\frac{3}{2} \alpha k x^{n} \dot{x}+k x^{n}=0
$$

Stiffness coefficients, as well as damping or impact restitution coefficients, will depend on material properties and bearing geometry.

\subsubsection{Catcher Bearing Interaction with Fluid Medium}

Catcher bearings will be placed in between the rotor and stator cans, which imply they will both be exposed to FLiNaK. For this reason, it is important that the corrosion behavior of the material is favorable. The corrosion of silicon carbide has been extensively studied at high temperatures and different environments; however, there are scarce data regarding corrosion in liquid fluoride salts. For this reason, the corrosion behavior of silicon carbide was inferred from studies in other environments. As shown in Fig. 34, studies show that in the presence of liquid salts, corrosion occurred at high oxygen partial pressures in the presence of basic salt melts and at very low oxygen partial pressures regardless of the basicity/acidity of the salt melts. Little corrosion (passivation) occurred at high oxygen partial pressures and in the presence of acidic or neutral salt melts. Given that FLiNaK is a strongly basic salt, it supports a higher degree of corrosion than other salts. ${ }^{43}$ Subsequent work, performed in support of the previously 
mentioned salt loop, has demonstrated good performance of silicon carbide for exposure at $650^{\circ} \mathrm{C}$ in FLiNaK for 90 days. (D. F. Wilson, private communication).

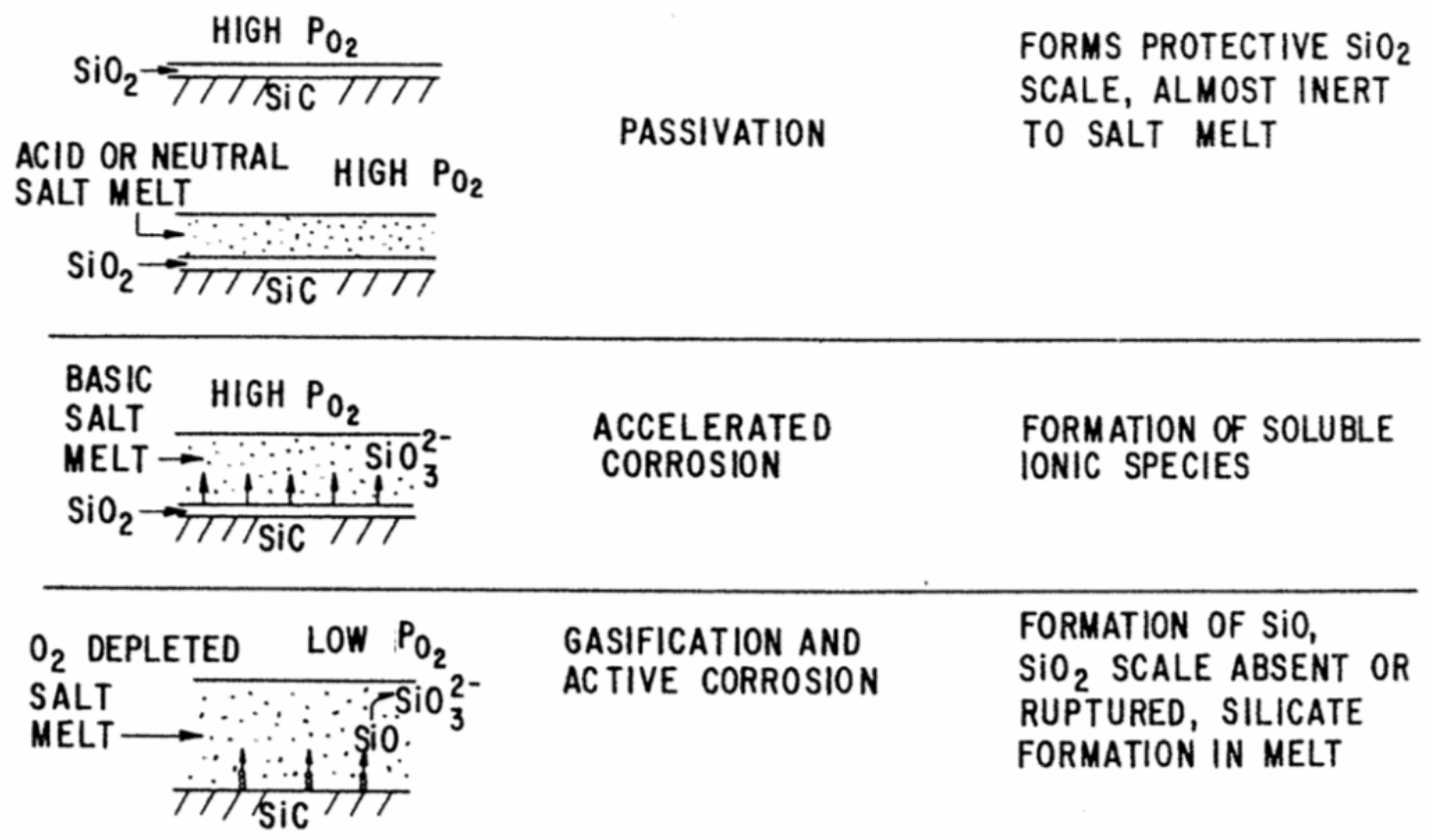

Fig. 34. Effects of salt on SiC (adapted from Ref. 44).

\subsubsection{Stiffness}

As described in Section 5.3.3, catcher bearings must withstand at least the weight of the rotor and impeller. Their ability to withstand the forces will depend on elasticity and damping characteristics of the bearing material. As silicon carbide is the most likely candidate for this application, some important parameters are given in Table 10.

Table 10. Silicon carbide bearing parameters. CRC Materials Science and Engineering Handbook

\begin{tabular}{ll}
\hline $\mathrm{SiC}$ Young's Modulus at $800^{\circ} \mathrm{C}$ & $365.52 \mathrm{GPa}$ \\
Graphite Young's Modulus & $9-13 \mathrm{GPa}$ \\
Bearing inner diameter & Refer to Section 0 \\
Bearing outer diameter & Refer to Section 0 \\
Bearing length & Refer to Section 0 \\
\hline
\end{tabular}

\subsection{ROTATIONAL SPEED AND DYNAMICS}

The torque ripple should not exceed 5 percent for $80-110$ percent rated pump speed. Torque ripple can be defined in various ways, and in this case, it is based on the quadratic mean of the ripple divided by the

\footnotetext{
${ }^{*}$ http://www.ceramisis.com/carbons_graphites_specs.htm.
} 
average torque multiplied by 100 . The torque ripple should not exceed 6 percent for $20-80$ percent rated pump speed. The forces on the rotor are analyzed in Table 11 (orientation shown in Fig. 35).

Table 11. Motor system force analysis

\begin{tabular}{|lcc|}
\hline \multicolumn{1}{|c|}{ Main Pump Forces } & Magnitude & Units \\
\hline Axial & 1.23 & $\mathrm{~N}$ \\
\hline \multicolumn{1}{|c|}{ Tangential } & & \\
\hline \multicolumn{1}{|c|}{ Viscous friction } & 23.45 & $\mathrm{~N}$ \\
\hline Torque (max) & 19.77 & $\mathrm{~N}$ \\
\hline Radial & 0.79 & $\mathrm{~N}-\mathrm{m}$ \\
\hline \multicolumn{1}{|c|}{ Forces on Shaft } & Magnitude & $\mathrm{N}$ \\
\hline Rotor weight & 232 & $\mathrm{Units}$ \\
\hline Impeller weight & 24 & $\mathrm{~N}$ \\
\hline Radial bearings & Controlled & $\mathrm{N}$ \\
\hline Pump Disturbance Forces & Magnitude (N) & Frequency (Hz) \\
\hline Shaft unbalance & & $0-60$ \\
\hline External vibration & 24.77 & 0.8 \\
\hline
\end{tabular}




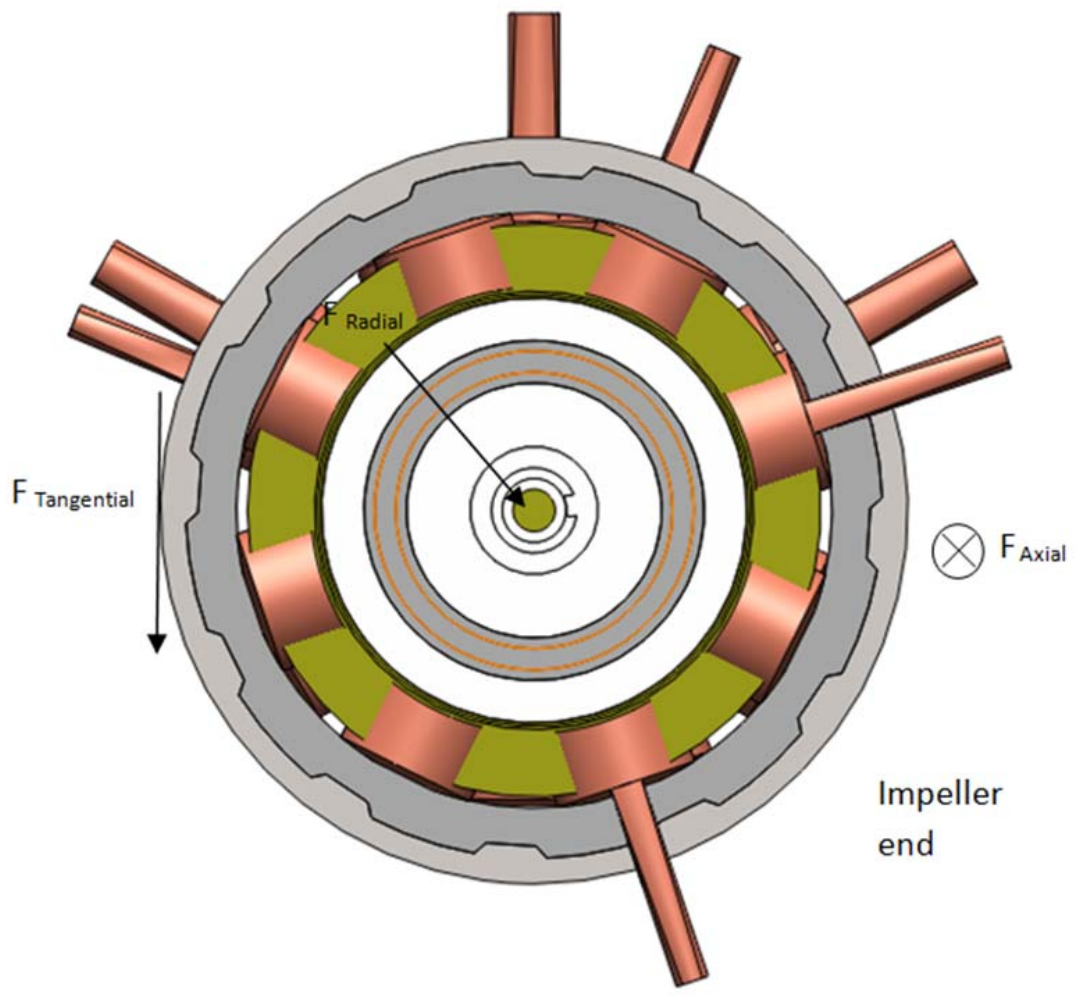

Fig. 35. Motor cross-sectional view showing force orientations.

The pump disturbance forces should not exceed \pm 10 percent of the pump forces shown with a bandwidth less than $2 \mathrm{~Hz}$.

\subsubsection{Design Life}

\section{Corrosion and salt-metal compatibility}

As several components of the canned rotor pump will be exposed to the fluid being pumped (FLiNaK), the corrosion behavior of the materials must be taken into account. The components that will be exposed to the fluid are as follows:

- rotor can

- stator can

- catcher bearings

- pump impeller

- pump body

- inner shaft

Materials that possess appropriate corrosion rates along with other functional requirements will be selected for use.

\section{Creep and erosion}

Metals subjected to loads over long periods of time and at elevated temperatures can undergo continuous deformation or creep. The canned rotor motor should last 7 years without the need of maintenance. For 
this reason, it is important to ensure that creep does not cause the materials to exceed the maximum allowable deformation over that period.

Hiperco ${ }^{\circledR} 50$, the material selected for the stator laminations, has been tested for creep for up to $593^{\circ} \mathrm{C}$ $\left(1100^{\circ} \mathrm{F}\right) .^{45}$ The creep strain-versus-time response curve for the material is shown in Fig. 36 for four different stress levels, from 30 to $60 \mathrm{ksi}$ in intervals of $10 \mathrm{ksi}$. The specimens were exposed to 30 and 40 ksi fractures at approximately 50 percent strain value, and lasted approximately 123 and 50 hours respectively. The exact strain values at fracture for $50 \mathrm{ksi}$ and $60 \mathrm{ksi}$ could not be recorded, but the fracture was recorded at 19.5 and 4.2 hours, respectively.

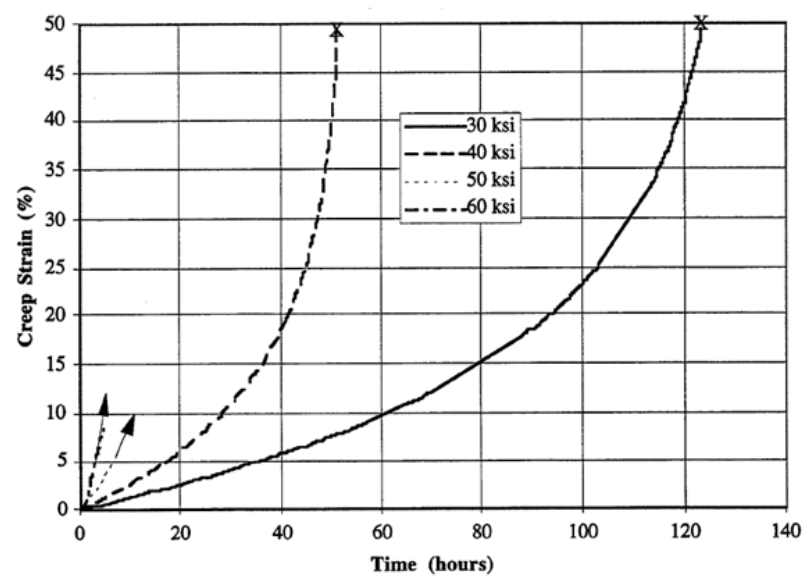

Fig. 36. HA50HS creep at $590^{\circ} \mathrm{C}$ (Source: Fingers, 1999). ${ }^{45}$

With experimental data at various temperatures and stresses, a plot of stress versus time for different temperatures and for a specified percentage creep strain can be generated. The plot of Fig. 37 shows the stress-versus-time curve for 1 percent creep strain from $371^{\circ} \mathrm{C}$ to $593^{\circ} \mathrm{C}\left(700^{\circ} \mathrm{F}\right.$ to $\left.1100^{\circ} \mathrm{F}\right)$. At higher temperatures, the stresses required to reach a specific strain by a given time are lower than those required at lower temperatures. A plot of stress versus time to fracture is also shown for Inconel ${ }^{\circledR} 600$ is shown in Fig. 38. 


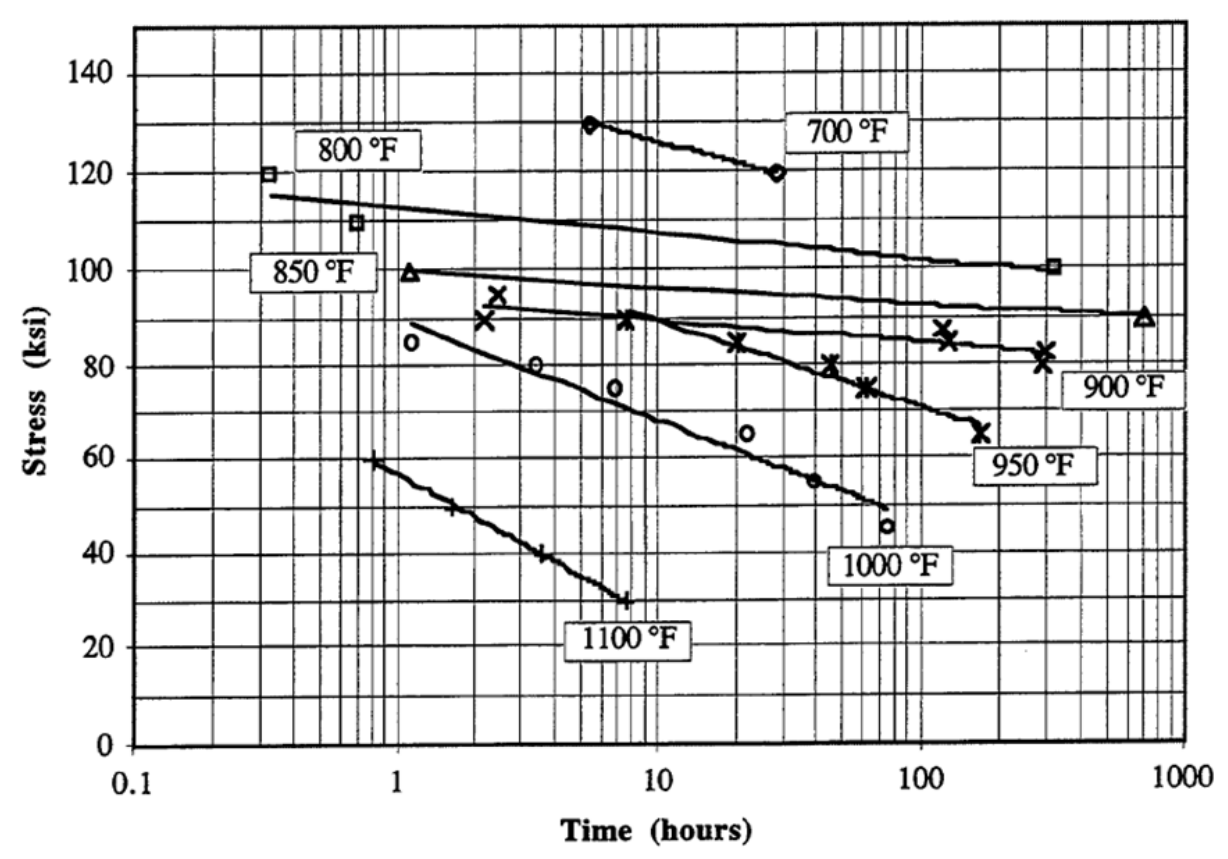

Fig. 37. Time to 1.0 percent creep strain (Source: Fingers, 1999). ${ }^{45}$

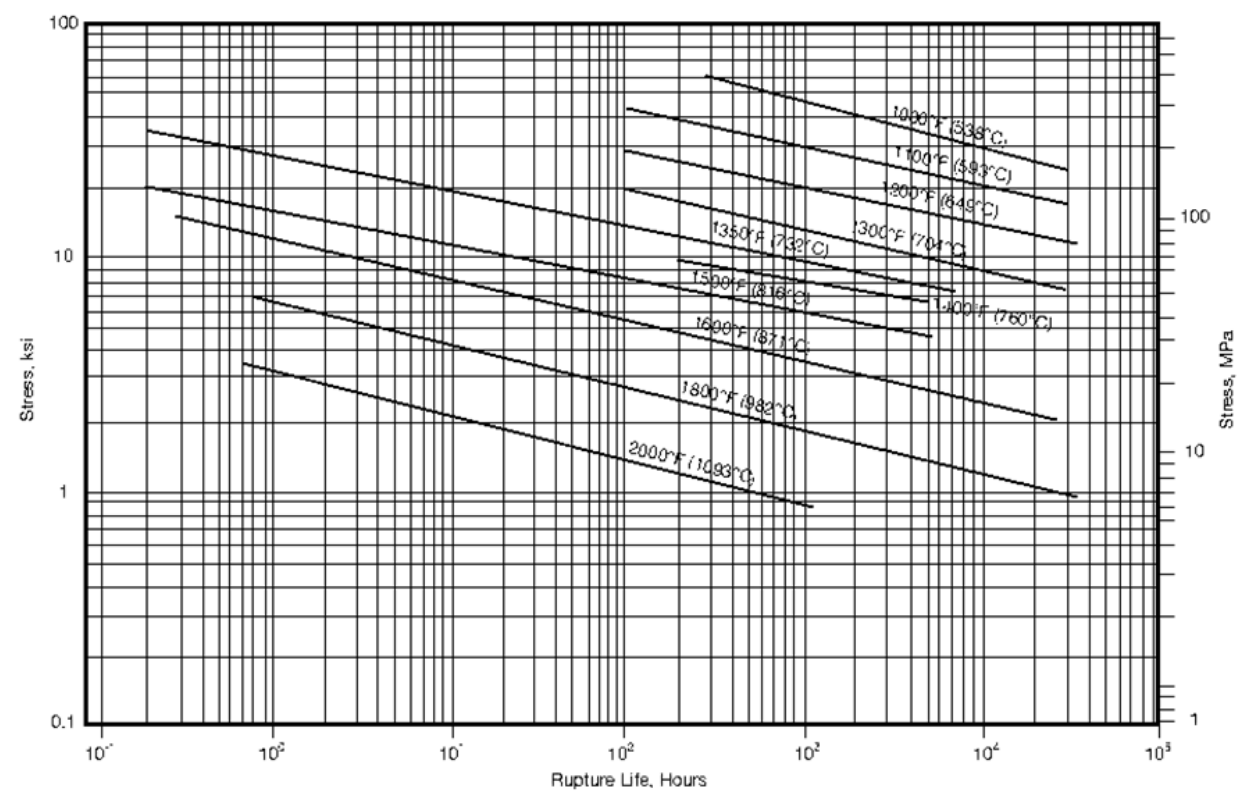

Fig. 38. Stress vs. time to fracture curve for Inconel ${ }^{\circledR} 600$ (Source: Special Metals) ${ }^{46}$

In the absence of a suitable data set for extrapolation via available models, some creep testing may be required for the selected motor materials at the particularly operating temperatures. The model can then be applied for determining the maximum stresses that can be applied before the specimen reaches a target percentage strain within the desired period. 


\subsection{ELECTRICAL AND MAGNETIC}

\subsubsection{Electrical Performance}

This section takes an in-depth look at the SRM electrical and magnetic performance for the pump motor application in which operation constrains the motor to relatively large air gap. The conventional SRM relies on small physical air gap separation between stator and rotor saliencies in order to maximize its inductance ratio of aligned to unaligned magnitude. As will be seen, this inductance ratio determines the back-EMF of the SRM during operation and its torque (i.e., tangential force) capability. Larger gaps introduce higher levels of leakage flux and thereby impose higher reactive burden on the power inverter, a topic considered in more detail in Section 5.5.2. Table 12 provides a definition of terminology of the SRM that will be used in the analysis and simulation work.

Table 12. SRM parameter definitions and initial estimates

\begin{tabular}{|c|c|c|c|c|c|c|c|}
\hline Parameter & Symbol & Value & Unit & Parameter & Symbol & Value & Uni \\
\hline Rotor diameter & $\mathrm{D}_{\mathrm{ro}}$ & 127 & $\mathrm{~mm}$ & $\begin{array}{l}\text { Rotor radius to tooth tip (w/o } \\
\text { rotor can) }\end{array}$ & $\mathrm{r}_{\mathrm{ro}}$ & 63.5 & $\mathrm{~mm}$ \\
\hline Maximum speed & $\mathrm{n}_{\mathrm{mx}}$ & 3600 & $\mathrm{rpm}$ & Continuous power rating & $\mathrm{P}_{\mathrm{r}}$ & 10 & $\mathrm{~kW}$ \\
\hline Continuous torque & $\mathrm{m}_{\mathrm{r}}$ & 26.5 & $\mathrm{~N}-\mathrm{m}$ & Rotor force & $\mathrm{F}_{\mathrm{r}}$ & 420 & $\mathrm{~N}$ \\
\hline Stator poles & $\mathrm{N}_{\mathrm{s}}$ & 6 & \# & Rotor poles & $\mathrm{N}_{\mathrm{r}}$ & 4 & \# \\
\hline Stack length (estimated) & $\mathrm{h}$ & 60 & $\mathrm{~m}$ & Working gap & $\mathrm{g}$ & 3 & $\mathrm{~mm}$ \\
\hline Stator phase resistance & $\mathrm{R}_{\mathrm{s}}$ & \# & $\Omega$ & Working temperature & $\mathrm{T}_{\mathrm{w}}$ & 700 & ${ }^{\circ} \mathrm{C}$ \\
\hline Stator lamination fill factor & $f_{h}$ & 0.94 & $\#$ & Stator winding fill factor & $\mathrm{f}_{\mathrm{cu}}$ & 0.25 & $\#$ \\
\hline Current density copper & $\mathrm{J}_{\mathrm{cu}}$ & 6 & $\mathrm{~A} / \mathrm{mm}^{2}$ & Stator pole angle & $\beta_{\mathrm{s}}$ & 0.55 & $\mathrm{rad}$ \\
\hline rotor pole angle & $\beta_{\mathrm{r}}$ & 0.524 & $\mathrm{rad}$ & $\begin{array}{l}\text { Constraint } \\
\left(\beta_{\mathrm{r}}+\beta_{\mathrm{s}}\right)<2 \pi / \mathrm{N}_{\mathrm{r}}=1.57\end{array}$ & & Yes & \\
\hline \multicolumn{4}{|c|}{ Preliminary 6/4 SRM design values } & \multicolumn{4}{|c|}{ Preliminary $6 / 4$ design values } \\
\hline Stator slot depth & $\mathrm{d}_{\mathrm{ss}}$ & 25 & $\mathrm{~mm}$ & Rotor slot depth & $\mathrm{d}_{\mathrm{rs}}$ & 15.5 & $\mathrm{~mm}$ \\
\hline Rotor shaft diameter & $\mathrm{D}_{0}$ & 40 & $\mathrm{~mm}$ & Rotor yoke iron depth & $d_{b r}$ & 28 & $\mathrm{~mm}$ \\
\hline Stator yoke iron depth & $\mathrm{d}_{\mathrm{bs}}$ & 18 & $\mathrm{~mm}$ & Number of turns/coil & $\mathrm{N}_{\mathrm{c}}$ & 24 & $\#$ \\
\hline Motor outer diameter & $\mathrm{D}_{\mathrm{s} 0}$ & 219 & $\mathrm{~mm}$ & Number in hand strands/turn & $\mathrm{NIH}$ & 12 & \# \\
\hline Coil winding gauge & AWG & 20 & $\#$ & Gap flux density - desired & $\mathrm{B}_{\mathrm{g}}$ & 0.6 & $\mathrm{~T}$ \\
\hline
\end{tabular}

Starting values for the rotor and stator pole pitch, $\tau_{\mathrm{r}}$ and $\tau_{\mathrm{s}}$, respectively, are given as Equation (49). In addition, for a large-gap SRM, a good starting position is to assign a rotor tooth width, $\mathrm{W}_{\mathrm{dt}}$, that is a third of the rotor pole pitch. For this situation, the rotor tooth width and initial stator tooth width can be assigned as Equation (50). This starting position results in a stator tooth width to a pitch of 52.5 percent, which is very close to the approximate geometry shown in Fig. 25. It is important to keep a small ratio on the rotor tooth width to pitch to minimize leakage (e.g., to hold a shallow slope on the unaligned flux linkage line shown), but to have sufficient winding volume in the stator slots. For this application, it may very well require rather deep stator slots to accommodate the necessary copper (ceramic insulated wire). Again, to minimize leakage, the stator poles (teeth) will be tapered to minimize saturation near the tooth base and thereby to minimize cross-slot leakage. Based on the values obtained in Equations (49) and (50), 
the required stator and rotor pole angles are calculated and stated in Table $19\left(31.5^{\circ}\right.$ and $30^{\circ}$, respectively).

$$
\begin{gathered}
\tau_{r}=\frac{2 \pi}{N_{r}} r_{r 0}=99.746 \mathrm{~mm} ; \tau_{s}=\frac{2 \pi}{N_{s}}\left(r_{r 0}+g\right)=69.638 \mathrm{~mm} . \\
W_{r t}=\frac{1}{3} \tau_{r}=33.248 \mathrm{~mm} ; \quad W_{s t} \cong 1.1 W_{r t}=34.817 \mathrm{~mm} .
\end{gathered}
$$

In the SRM, the flux linkage, $\lambda_{\mathrm{s}}(\theta, \mathrm{i})=\mathrm{L}(\mathrm{q}, \mathrm{i}) * \mathrm{I}$, where I is stator current, is regulated by the power inverter according to the desired torque and rps information. Figure 39 illustrates how driving the SRM stator core into saturation actually improves the torque production capability by maximizing the swept area enclosed by the solid trace shown, once per phase per cycle. In the high-temperature pump motor application, the minimum inductance (i.e., unaligned) is anticipated to be rather high due to a large gap. Figure 46 illustrates the role that inductance variation and maximum flux linkage play in energy conversion.

$$
W_{m x}=\frac{1}{2} \lambda_{m x}^{2}\left(\frac{1}{L_{m n}}-\frac{1}{L_{m x}}\right) .
$$
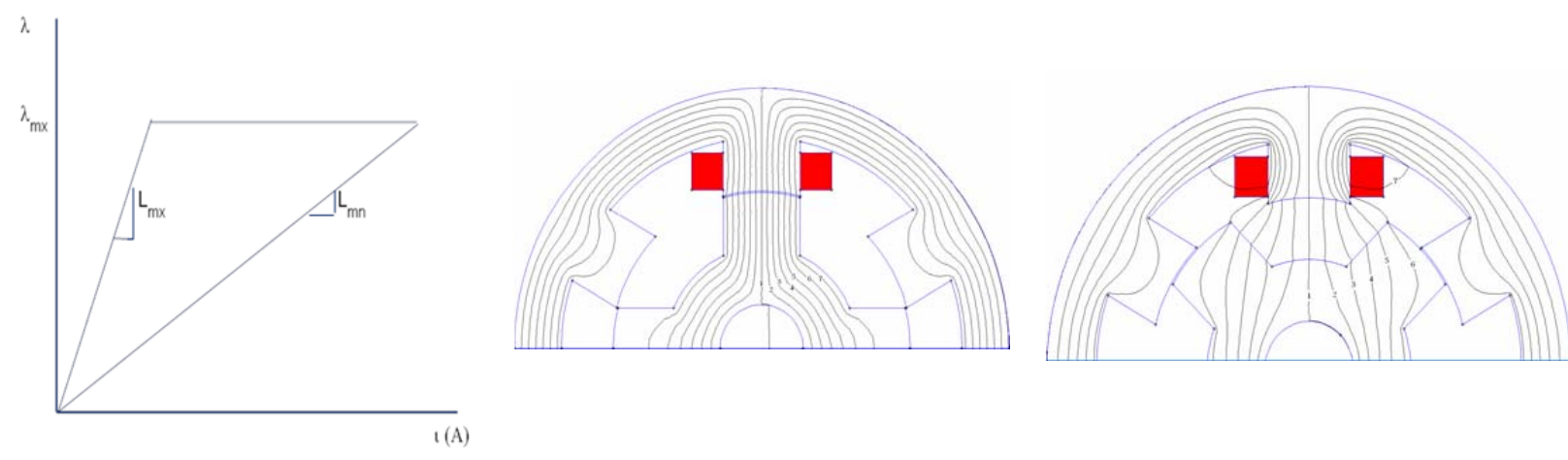

Fig. 39. SRM energy conversion for large gap design. Center: 6/4 SRM aligned, Right: Unaligned.

Based on the preliminary design values listed in Table 19 for the 6/4 SRM, an initial assessment was made for the phase flux that could be delivered based on the iron and copper fill factors listed. A magnetic equivalent circuit model (MEC) was used to make these estimates. The key parameters are summarized here in Equation (52):

$$
\begin{aligned}
& \sum R_{x}=2 R_{s t}+2 R_{g}+2 R_{r t}+\frac{R_{r y}}{2}+\frac{R_{s y}}{2} \\
& \sum R_{x}=6.9 \times 10^{4}+200 \times 10^{4}+4.5 \times 10^{4}+9.2 \times 10^{4}+42.6 \times 10^{4}=3.05 \times 10^{6}\left(\frac{A}{W b}\right)
\end{aligned} .
$$


Referring to Equation (52), it is clear that with $\mathrm{g}=3 \mathrm{~mm}$ gap at each stator-rotor pole overlap the aligned path reluctance (see center Fig. 46) is extremely high in terms of nominal $\mathrm{g} \sim \mathrm{r}_{\mathrm{ro}} / 100=0.5$ to $0.7 \mathrm{~mm}$ gaps. In fact, in this SRM design if $\mathrm{g}=0.7 \mathrm{~mm}$, the term $2 * \mathrm{R}_{\mathrm{g}}=50.6 \times 10^{4} \mathrm{~A} / \mathrm{Wb}$ and therefore very comparable to the reluctance of the stator yoke flux return paths combined. Using the aligned reluctance and the design value $\mathrm{N}_{\mathrm{c}}=24$ turns/coil and assigning a desired gap flux density, $\mathrm{B}_{\mathrm{g}}=0.6 \mathrm{~T}$, then based on calculated gap area, the total pole flux in the gap due to excitation of one phase is $\phi_{\mathrm{g}}=\mathrm{B}_{\mathrm{g}} * \mathrm{~A}_{\mathrm{g}}=1.32$ $\mathrm{mWb}$. Based on this information, the continuous duty phase current, $\mathrm{I}_{\mathrm{s}}$, can be derived as Equation (53).

$$
F_{m}=2 N_{c} I_{s} ; \quad \therefore \quad I_{s}=\frac{\varphi_{g} \sum R_{x}}{2 N_{c}}=83.88 \mathrm{~A} .
$$

This is a very reasonable phase current for this machine; however, it is insufficient to make any conclusions on torque or power capability. It is necessary to have machine values for the inductances listed in Equation (51) and Fig. 46. Following up on the aligned case, it is easy to compute the aligned inductance, $\mathrm{L}_{\mathrm{a}}(\theta, \mathrm{i})$, simply as Equation (54):

$$
L_{a}\left(0, I_{s}\right)=\frac{2 N_{c}^{2}}{\sum R_{x}}=0.460 \mathrm{mH} .
$$

The aligned flux linkage, or maximum flux linkage, $\lambda_{\mathrm{mx}}$, is derived directly from Equation (54) as

$$
\lambda_{m x}\left(0, I_{s}\right)=2 N_{c} \varphi_{g}=63.36 \mathrm{mWb} \text {. }
$$

The unaligned inductance can be estimated based on the SRM rotor position shown on the right in Fig. 46 , where the stator pole angle, $\beta_{\mathrm{s}}=30^{\circ}$, and the unaligned rotor angle, $\beta_{u}$, is the angle between each side of this stator pole and the adjacent rotor pole sides. Using values shown in Table 19, the unaligned angle $\beta_{u}=14.3^{\circ}$. The approximate unaligned inductance is given as

$$
L_{u}=\frac{F_{f} \mu_{0}\left(2 N_{c}^{2}\right) h f_{F e} r_{r 0} \beta_{s}}{r_{r 0} \beta_{u}}=0.2 \mathrm{mH}
$$

Based on the unaligned inductance, the energy conversion per stroke [see Equation (51)] can be derived and from this the torque and power. Generally, the rated power is defined at the point when the SRM back EMF (BEMF) equals the dc bus voltage, $U_{d 0}$. The power of an SRM at $\eta=100$ percent efficiency depends strongly on its power factor (PF), which is limited to $2 / 3$ at 100 percent efficiency and lower as the efficiency drops.

$$
\begin{aligned}
B E M F & =2 N_{c} r_{r 0} h f_{F e} B_{s a t} \omega_{m} . \\
P_{m} & =\frac{3}{2} U_{d 0} I_{s} P F \eta
\end{aligned}
$$

For a large gap SRM, the inductance ratio is going to be very limited and not near the nominal $6 \mathrm{x}$ to $10 \mathrm{x}$ obtained in small gap ( 0.5 to $0.7 \mathrm{~mm}$ ) designs. This limitation can be seen from the above analysis, where the reluctance of the iron paths is 34 percent of the total flux path reluctance fully aligned and partially saturated steel, but it is less than 2 percent of the total reluctance unaligned. For example, for the pole dimensions tabulated, the gap reluctance, aligned position, and $\mathrm{g}=0.7 \mathrm{~mm}, \mathrm{R}_{\mathrm{g}}=2.53 \times 10^{5} \mathrm{~A} / \mathrm{Wb}$ 
increasing to $\mathrm{R}_{\mathrm{g}}=10.85 \times 10^{5}$ when $\mathrm{g}=3 \mathrm{~mm}$ and $\mathrm{R}_{\mathrm{gu}}=7.8 \times 10^{6}$ when $\mathrm{g}=3 \mathrm{~mm}$ and unaligned. Note that for this initial SRM design, the ratio of aligned to unaligned inductances, $\xi=\mathrm{L}_{\mathrm{a}} / \mathrm{L}_{\mathrm{u}}=\mathrm{L}_{\mathrm{mx}} / \mathrm{L}_{\mathrm{mn}}=2.345$, which will be rounded up to 2.5 in the next section.

\subsubsection{Voltage, current, phases}

The induced voltage in the SRM is the result of inductance changes with rotor position and the amount of current in the phase coils [Equation (59)]. Electrical power input under motoring mode is then the product of this induced, or BEMF, voltage and the current that produced it. This electrical input power in turn is composed of power dissipated in the phase coil resistance, $r_{s}$, the derivative of stored energy and the mechanically converted energy at constant current (power inverter regulated current).

$$
\begin{gathered}
U_{p h}=r_{s} i+\frac{d}{d t}\{L(\theta, i) i\}=r_{s} i+L(\theta, i) \frac{d i}{d t}+i \frac{d L(\theta, i)}{d \theta} \frac{d \theta}{d t} \\
P_{e}=U_{p h} i=r_{s} i^{2}+i L(\theta, i) \frac{d i}{d t}+i^{2} \frac{d L(\theta, i)}{d \theta} \omega .
\end{gathered}
$$

It is instructive to consider an example case SRM to appreciate the role inductance plays in the operation of this electric machine. For linear operation, such as the unaligned flux linkage trajectory shown in Figs. 45 and 46, the inductance values for a three-phase SRM can be written as a biased sinusoid that has a variation given by the number of rotor saliencies multiplied by the mechanical angle of the rotor.

$$
a \equiv \frac{2 \pi}{3}
$$

$$
\begin{aligned}
& L_{a}(\theta, i)=L_{0}+\delta L \cos \left(N_{r} \theta\right) \text {; } \\
& L_{b}(\theta, i)=L_{0}+\delta L \cos \left(N_{r}(\theta+a)\right) ; \quad L_{c}(\theta, i)=L_{0}+\delta L \cos \left(N_{r}(\theta-a)\right) \\
& i_{a}(\theta)=I_{s} \forall \theta \varepsilon[45,90] \&[135,180] ; \\
& i_{b}(\theta)=I_{s} \forall \theta \varepsilon[-15,30] \&[75,120] \&[165,210] ; \text { etc } .
\end{aligned}
$$

The inductance variation with angle is shown in Fig. 40 for illustration along with representative phase current pulses set to coincide with the interval when $\mathrm{dL} / \mathrm{d} \theta>0$. The choice of rectangular phase pulse is consistent with a practical SRM power inverter operating under PWM current control for the particular speed and winding inductance variation with angle. 

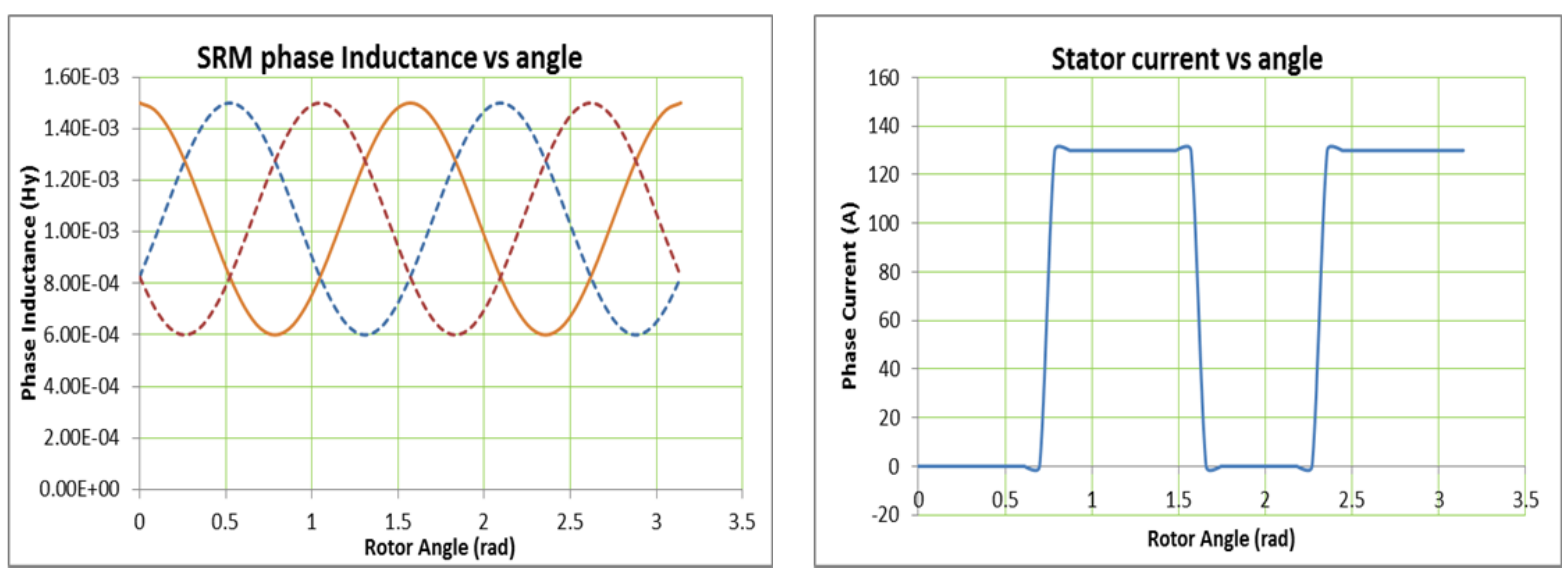

Fig. 40. SRM inductance variation (A-phase solid trace) and representative phase-A current pulse.

In this example, the phase inductance of the SRM is selected to have maximum aligned value of $1.5 \mathrm{mH}$ and minimum value of $0.6 \mathrm{mH}$, as shown in Fig. 40. When the phase current amplitude is set to $\mathrm{I}_{\mathrm{s}}=130$ A, the resulting power versus angle according to Equation (53) takes the shape shown in Fig. 41.

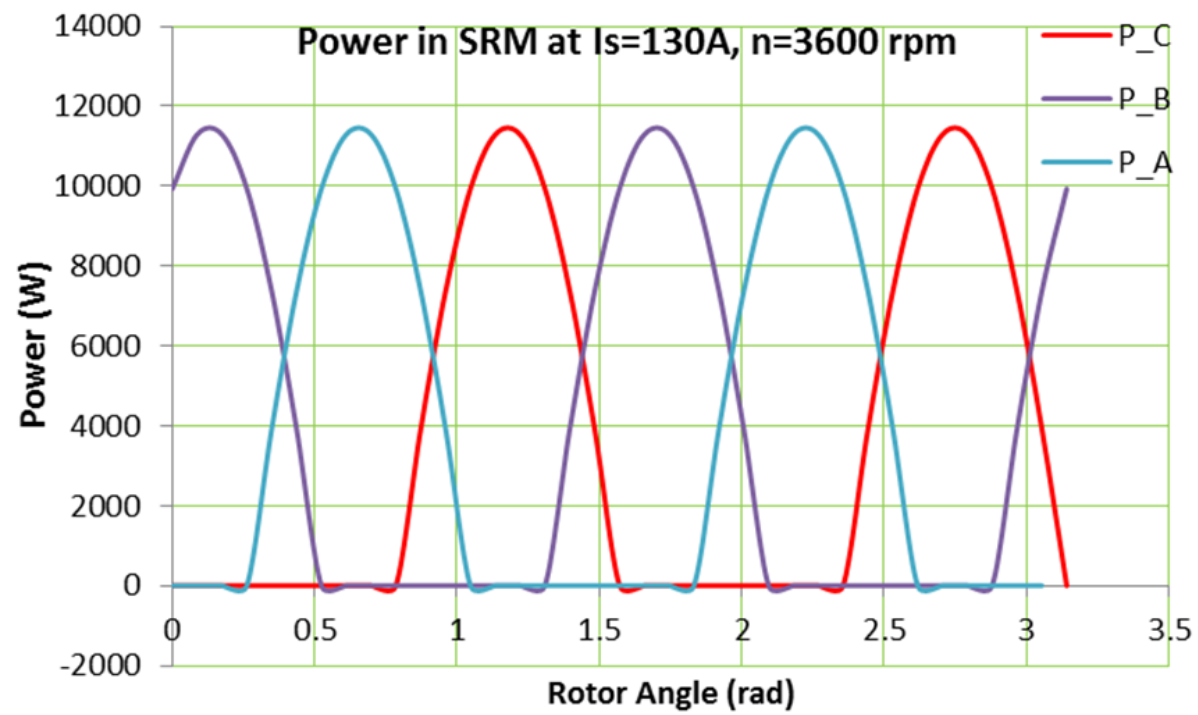

Fig. 41. SRM phase power pulses for rectangular current input when $\mathbf{n}=3600 \mathrm{rpm}$.

The performance shown here is representative only and meant to illustrate the need for as large an inductance variation with position as possible. In this case the ratio of $\xi=\mathrm{L}_{\mathrm{mx}} / \mathrm{L}_{\mathrm{mn}}=2.5$, which would be the case when the flux linkage aligned to unaligned traces in Fig. 46. It is the objective of this program to design an SRM that optimizes the inductance ratio $\xi$ in order to deliver the maximum rotor force, $F(\theta)$, as possible.

\subsubsection{Magnetic performance}

This section presents a series of finite-element analysis (FEA) results on the 6/4 SRM with small (1 mm) and rated $(3 \mathrm{~mm})$ air gaps. This gap does not include effects of stator and rotor conducting cans needed for shielding from the working medium. Stator current is incremented to $200 \mathrm{~A}$ in $25 \mathrm{~A}$ steps for the evaluation of rotor torque versus position. The two charts in Fig. 42 (top right, bottom left) are torque 
versus position. Simulation indicates that the $3-\mathrm{mm}$ gap reduces torque by a factor of $1 / 3$ for low currents, but only about $2 / 3$ for higher currents as shown in Fig. 42 (bottom right). Notice in the two charts for $\mathrm{m}\left(\theta, \mathrm{I}_{\mathrm{s}}\right)$ that $\delta \mathrm{m}\left(\theta, \mathrm{I}_{\mathrm{s}}\right) / \delta \mathrm{Is}$ is almost constant when $\mathrm{g}=1 \mathrm{~mm}$ but increases in the $\mathrm{g}=3 \mathrm{~mm}$ case due to the significant influence saturation has on SRM performance. That is, the SRM benefits strongly from deep saturation of the soft iron components. This is consistent with Figs. 41 and 42, showing that the energy converted per stroke grows as the SRM is pushed deeper into saturation.
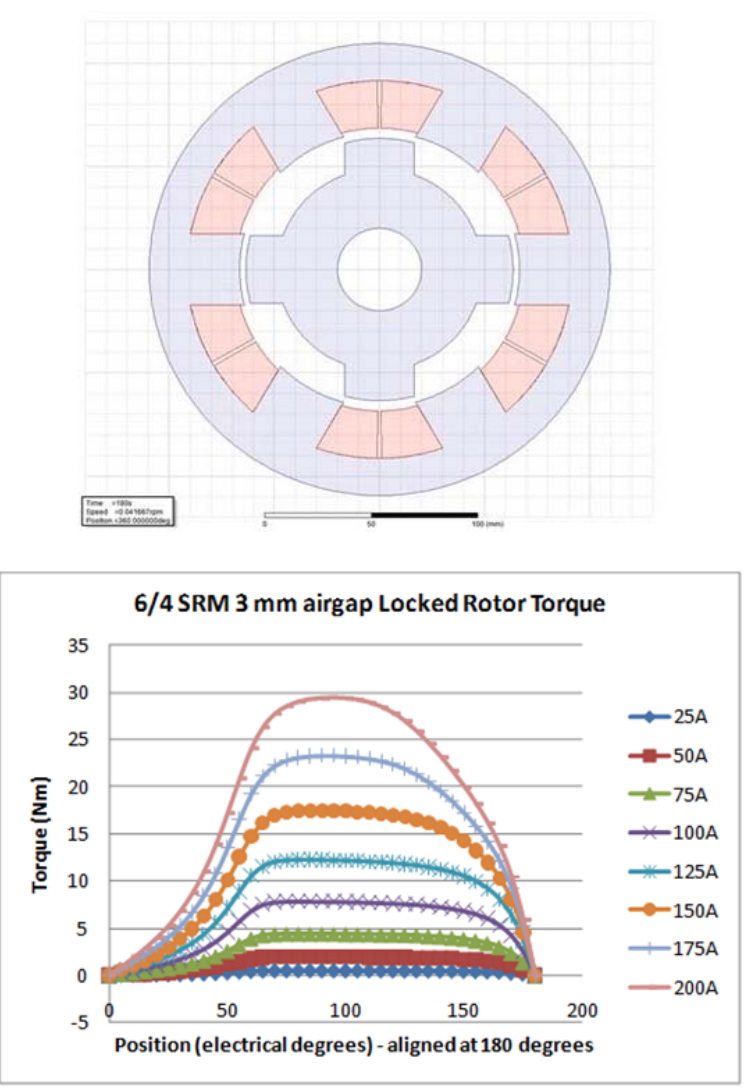
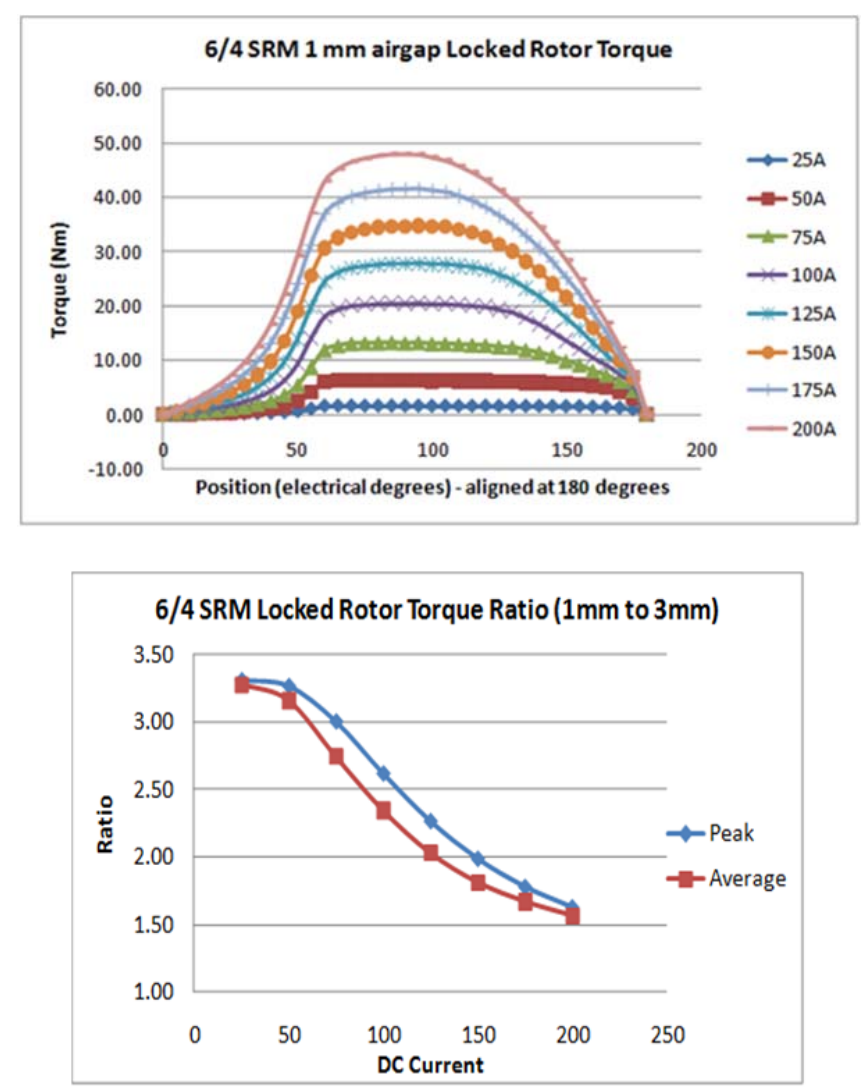

Fig. 42. Top Left: SRM simulated torque vs rotor angle, $\theta_{\mathrm{r}}=\mathbf{0}^{\circ}$ in fully unaligned position and $\theta_{\mathrm{r}}=180^{\circ}$ electrical in fully aligned position relative to phase $A$ (as shown). Top right: $\mathbf{m}\left(\theta, I_{s}\right)$ when $\mathrm{g}=1 \mathrm{~mm}$, bottom left: $\mathrm{m}\left(\theta, \mathrm{I}_{\mathrm{s}}\right)$ when $\mathrm{g}=3 \mathrm{~mm}$, and bottom right: $\mathrm{m}_{\mathrm{pk}}\left(\theta, \mathrm{I}_{\mathrm{s}}\right)$ for $\mathrm{g}=1 \mathrm{~mm} \mathrm{vs} . \mathrm{g}=3 \mathrm{~mm}$ case.

\subsubsection{Stator normal operations}

The most significant feature of the SRM is that in normal operation it must be driven well into saturation for highest energy conversion and for minimizing its power factor (PF), which minimizes the power inverter $\mathrm{kVA} / \mathrm{kW}$ rating. Figure 43 extends the model and simulation performance shown in Fig. 42 above for comparison of the SRM peak to average torque per ampere as a function of stator-rotor pole (tooth) gap. This again does not include the influence of conducting cans on the stator bore and rotor periphery.

The results in Fig. 43 show that for small gap the SRM torque/amp becomes a constant above a certain level of current injection, but for the large gap SRM the torque/amp increases linearly over a greater range of current. This result is because for a large gap the soft iron paths are not going into deep saturation. 

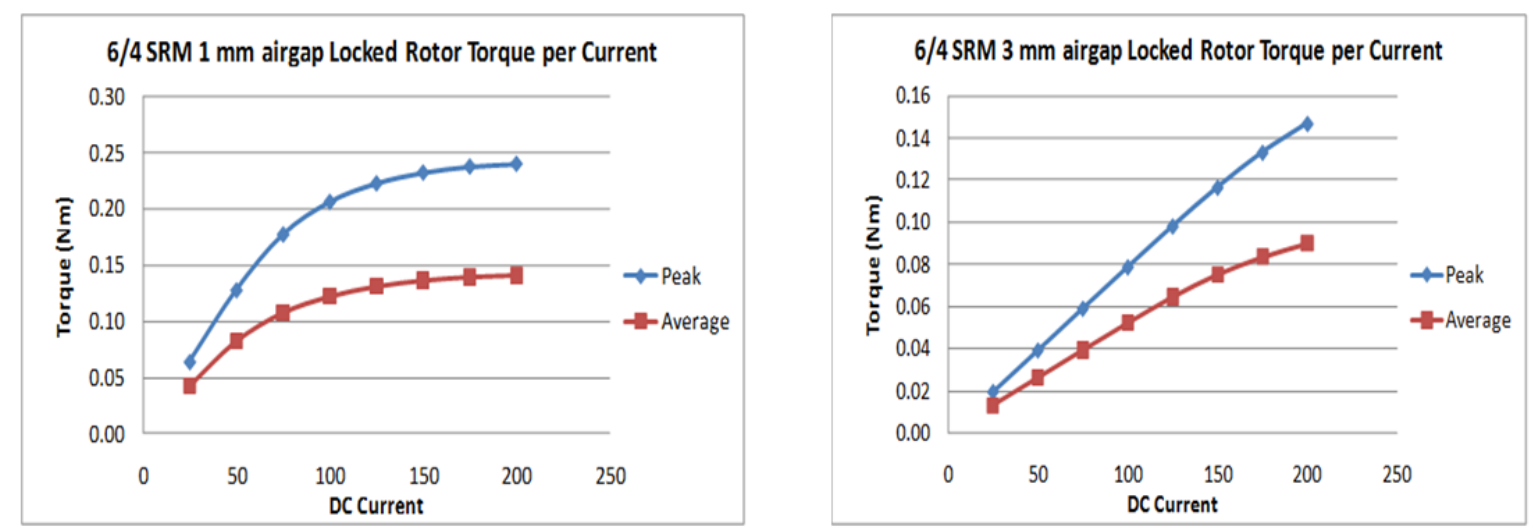

Fig. 43. Left: SRM simulated torque/amp for $\mathrm{g}=1 \mathrm{~mm}$; right: torque/amp for $\mathrm{g}=3 \mathrm{~mm}$.

\subsubsection{Rotor normal operations}

This section expands on the developments following Table 12, the baseline 6/4 SRM design, to treat an optimized SRM having somewhat narrower rotor poles (saliencies, teeth). In this analysis, as in the above section, the base parameters are those summarized in Table 12. Locked rotor simulations were conducted with dc current levels of $25 \mathrm{~A}$ to $200 \mathrm{~A}$ in $25 \mathrm{~A}$ increments as before, with various rotor and stator tooth widths, where the pertinent dimensions are those listed in Figs. 44 and 45. In subsequent analysis, nominal angular width of the rotor tooth is defined as $\beta \mathrm{r} \_$nominal $=2 * \mathrm{pi} /(\mathrm{Ns} * 2)$, where $\mathrm{Ns}$ is the number of stator teeth. The nominal width of the rotor tooth is $\overline{\mathrm{Wr}} \_$nominal $=2 * \mathrm{r}_{-}$rotor* $\sin (\mathrm{Br} / 2)$. The adjusted angular width can be defined as $\beta r=\mathrm{Krtr} * \mathrm{Br} \_$nominal. The adjusted rotor tooth width is $\mathrm{Wr}=2 * r_{-}$rotor*sin $(\mathrm{Krtr} * \mathrm{Br} / 2)$. The nominal stator tooth width can be defined with respect to the adjusted rotor tooth width as Ws_nominal $=$ Wr. Similarly, the adjusted width of the stator tooth can be defined as Ws $=$ Kstr*Ws_nominal $=$ Kstr*Wr. The angular width of the stator tooth tip is $\beta \mathrm{s}=2 * \operatorname{asin}\left(\mathrm{Ws} / 2 / \mathrm{r}_{-} \mathrm{stator}\right.$ inner$)$.

The definition of $\beta$ s seems unnecessarily complicated, but this was done so that for $\mathrm{Krtr}=1$ and $\mathrm{Kstr}=1$, the width of the stator and rotor teeth are equal as done in the baseline design. If the air gap is relatively large, as it is here, the tooth widths are somewhat different from when using a nominal 30 degree arc length to define rotor and stator tooth width.

Torque data from magnetics FEA simulation were generated for all combinations of Krtr and Kstr as these parameters ranged from 0.6 to 1.4 and the rotor position varied from 0 to 180 electrical degrees in 5 degree increments, giving 23,976 data points for each air gap size. 


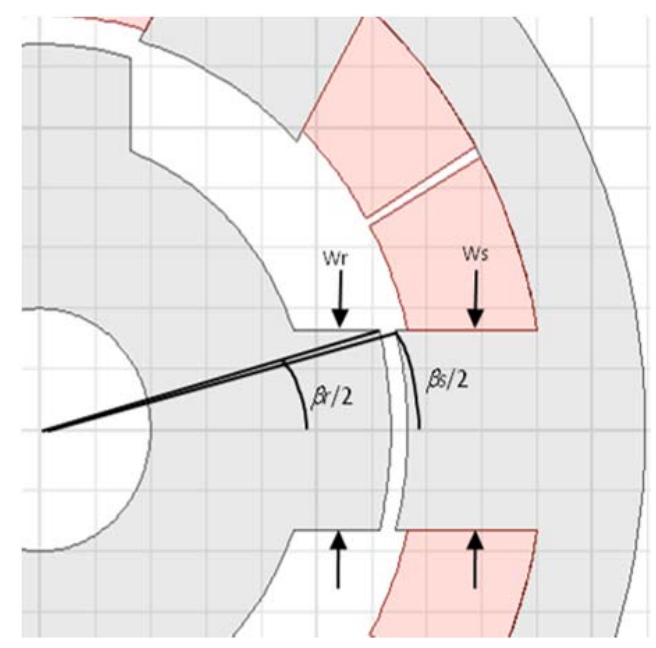

Fig. 44. Baseline design with $\mathrm{Krtr}=\mathrm{Kstr}=1$.

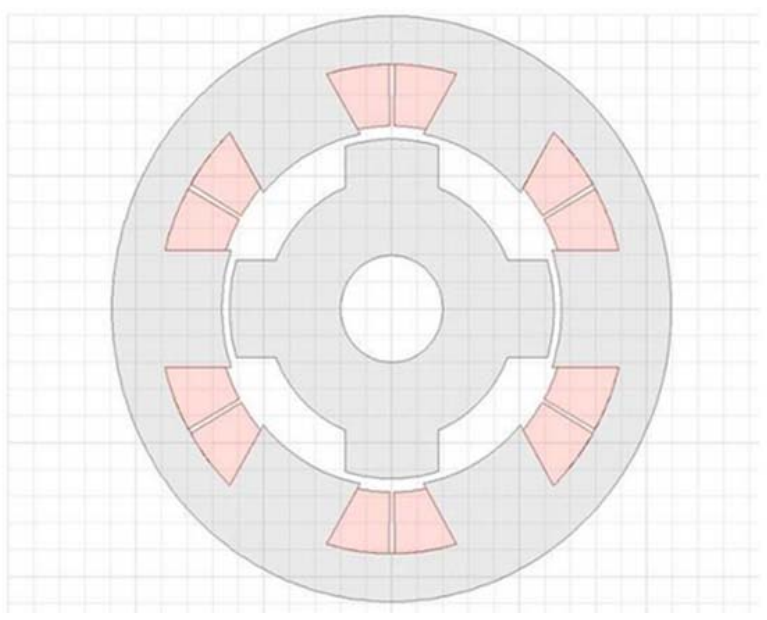

Fig. 45. SRM optimized design Krtr $=1.1125$ and $\mathrm{Kstr}=1.2$

Figure 46 shows the results for one design scenario with $\mathrm{Krtr}=1$ and $\mathrm{Kstr}=1.05$ with an air gap of $1 \mathrm{~mm}$. To study the impact of tooth width upon torque capability, torque data for each design scenario was averaged over the 180 degrees for each current level. Contour plots were generated for each current level so that the maximum average locked rotor torque is easily visible. Results of this iteration are shown in Figs. 47 and 48 as torque contour maps at fixed dc current of $200 \mathrm{~A}$ and for air gaps of $1 \mathrm{~mm}$ and $3 \mathrm{~mm}$, respectively. As the superimposed text boxes indicate, maximum average locked rotor torques of $29.5 \mathrm{~N}-\mathrm{m}$ and $19.5 \mathrm{~N}-\mathrm{m}$, respectively, are obtained with $\mathrm{Krtr}=1.05$ and $\mathrm{Kstr}=1.1625$ for a $1 \mathrm{~mm}$ air gap and $\mathrm{Krtr}=1.1125$ and $\mathrm{Kstr}=1.2$ for a $3 \mathrm{~mm}$ air gap.

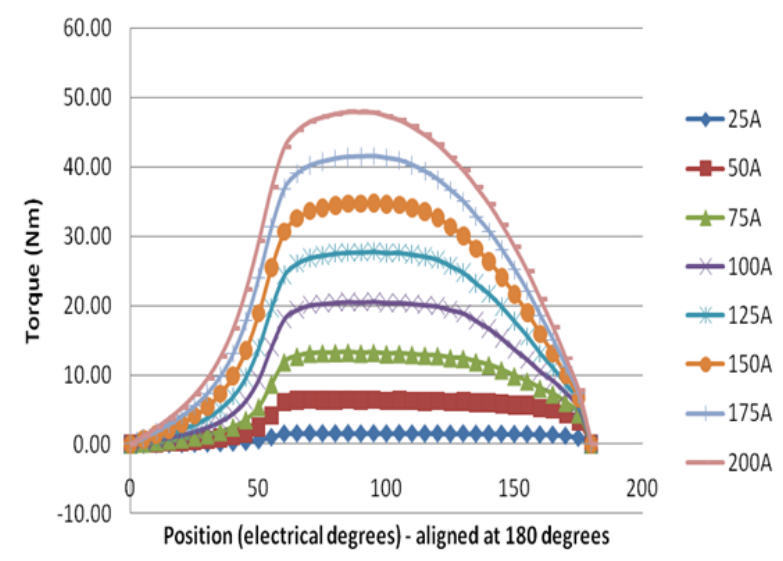

Fig. 46. SRM locked rotor torque evaluation when $\mathrm{Krtr}=1$, Kstr $=1.05$, and $\mathrm{g}=1 \mathrm{~mm}$. 


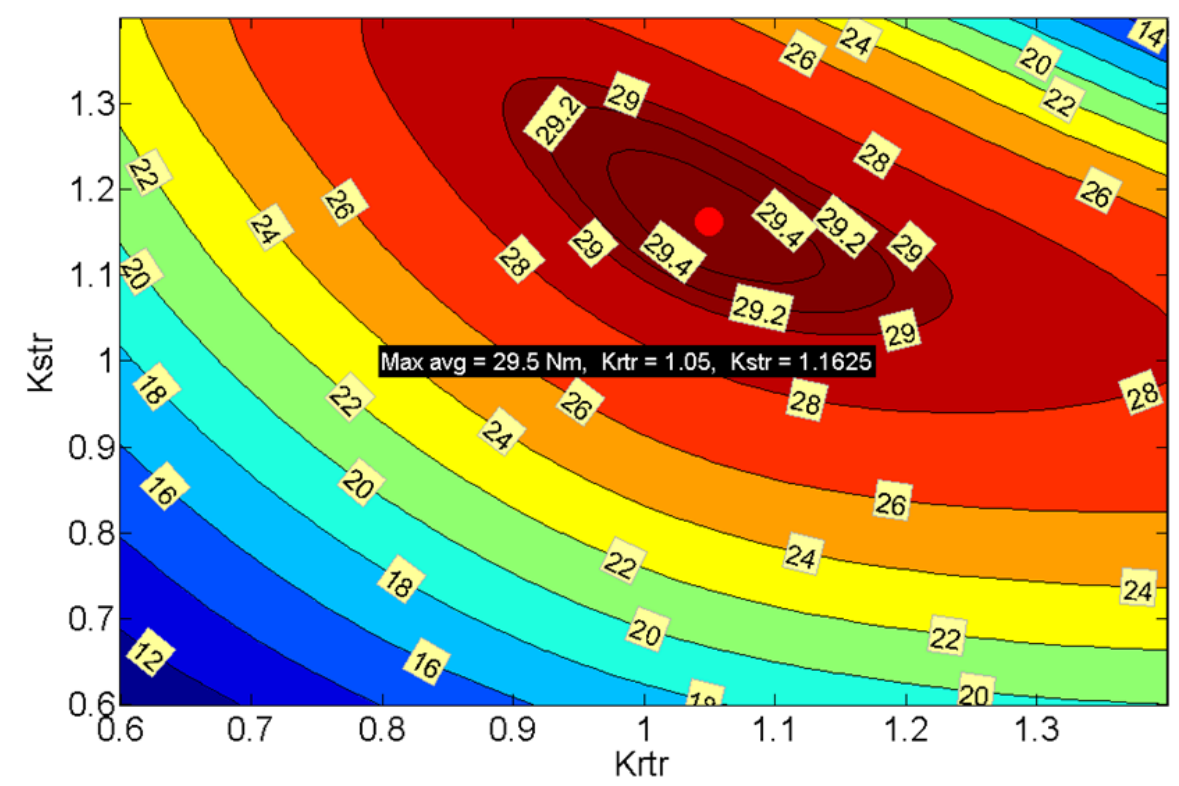

Fig. 47. SRM average locked rotor torque at $I s=200 \mathrm{~A}$ and $\mathrm{g}=1 \mathrm{~mm}$ over spread of Krtr and Kstr.

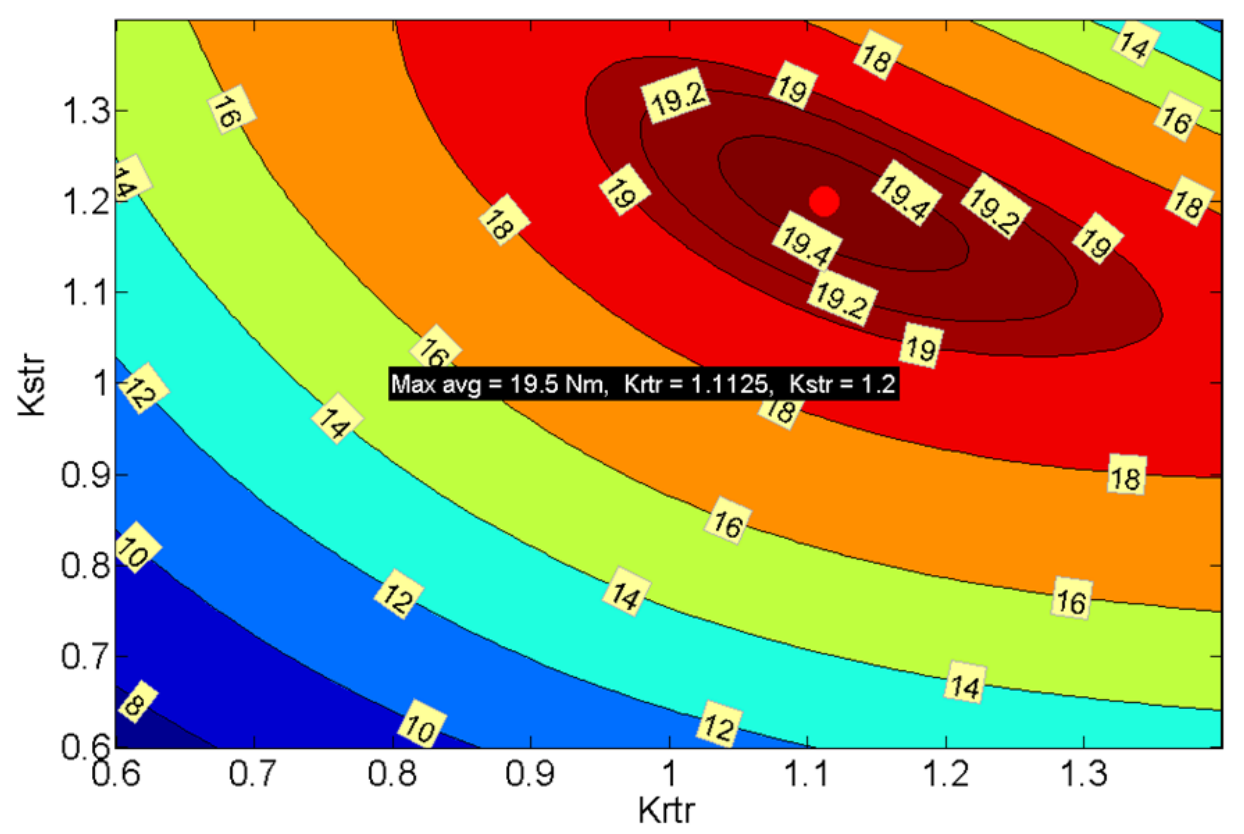

Fig. 48. SRM average locked rotor torque at $I s=200 \mathrm{~A}$ and $\mathrm{g}=3 \mathrm{~mm}$ over spread of Krtr and Kstr.

Tooth widths that correspond to maximum developed torque for each DC current are indicated in Fig. 49, where it is apparent that the optimal rotor and stator tooth width vary with operation current, and thus the applied load. It is recommended that rotor and stator tooth widths be chosen such that torque per unit current is maximized near the anticipated continuous operating point of the machine. 


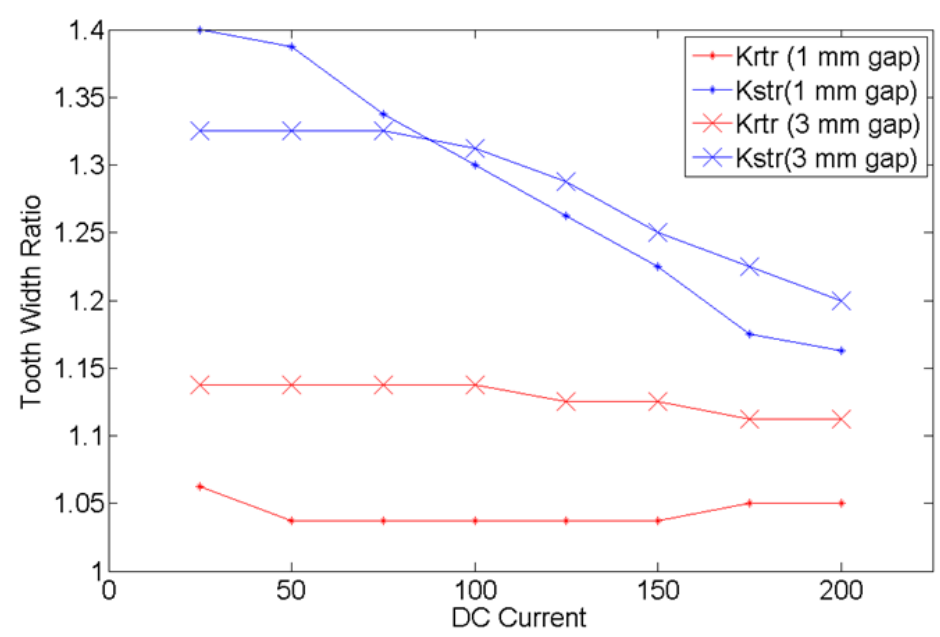

Fig. 49. Tooth widths for maximum average locked rotor torque for $\mathrm{g}=1 \mathrm{~mm}$ and $3 \mathrm{~mm}$.

In the comparison of optimal tooth width ratios, Krtr and Kstr, that rotor tooth width need not vary much from the baseline calculation of $30^{\circ}$, but that stator tooth width is optimized with current, being somewhat narrower (stator slot width widens) as stator current exceeds $100 \mathrm{~A}$.

It is also recommended at this stage of design to investigate multiple-phase excitation-phase torque optimization, such as bipolar drive versus unipolar current drive (discussed thus far). This recommendation is driven by two considerations:

(1) brief flux shunt during phase commutation and

(2) enhanced output from bipolar drive.

Figures 50 and 51 illustrate the case for both points and present a six-switch power converter having midpoint to SRM phase winding neutral clamping. Power electronics for bipolar drive of SRM is illustrated in Fig. 52.

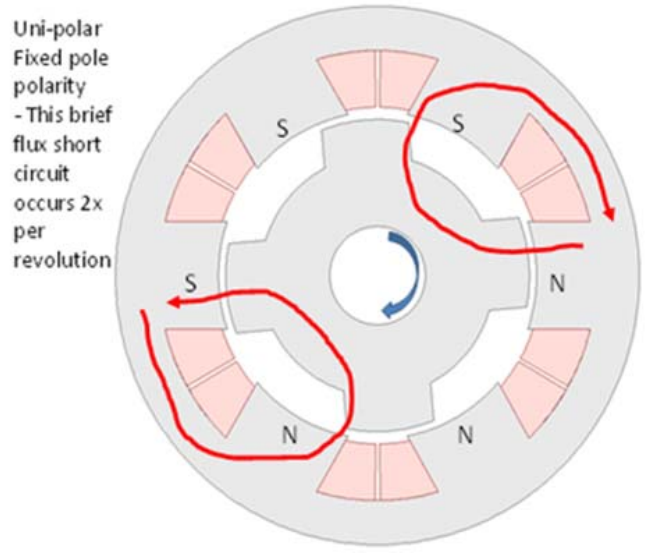

Fig. 50. Flux shunt during unipolar commutation.

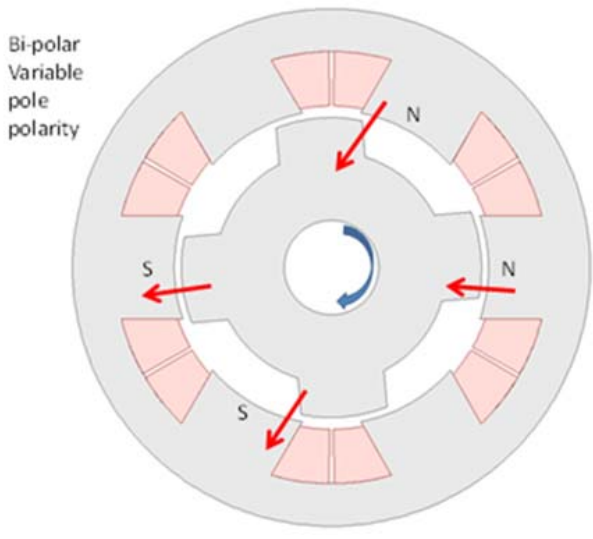

Fig. 51. Flux paths during bipolar drive. 


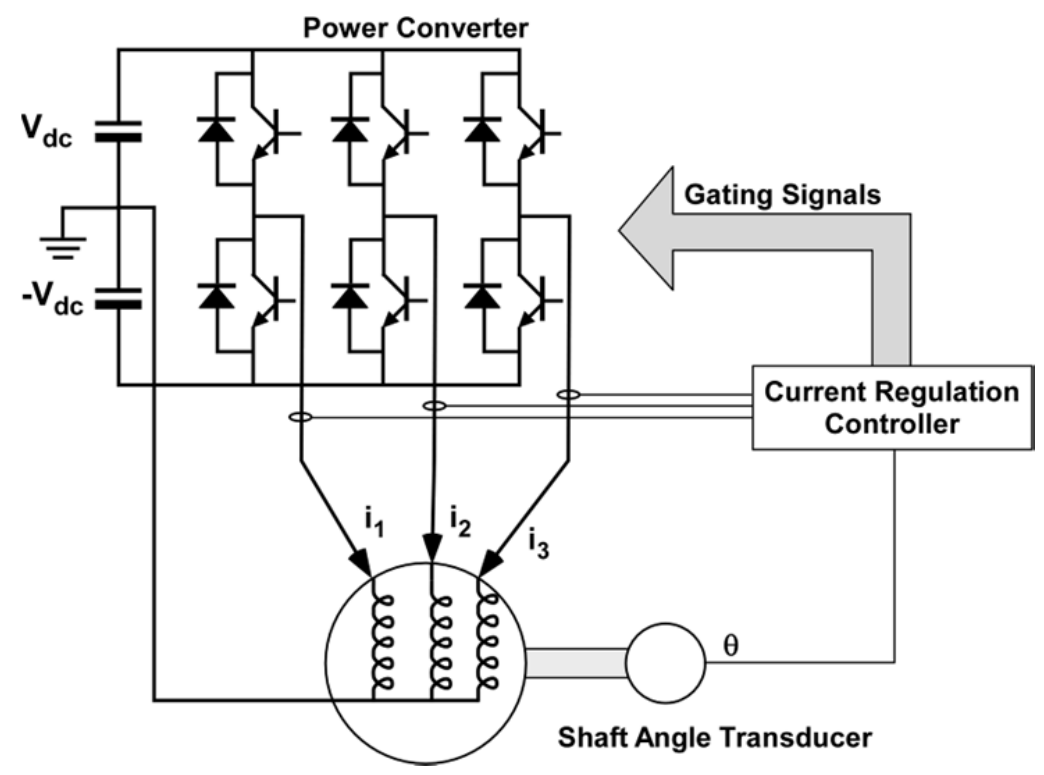

Fig. 52. Power electronics circuit topology for bipolar drive of SRM.

\subsection{RADIAL POSITION AND SPEED SENSORS}

From a parts minimization perspective, the preferred sensor technology uses the existing drive windings to derive the rotor's radial position. This sensorless approach is more difficult to implement than utilizing dedicated sensors. Traditional position detection in motors uses separate sensors.

\section{Angular position sensing}

Optimal and stable control of switched reluctance motors requires accurate rotor position feedback in order to energize and de-energize stator windings in proper sequence and at the appropriate times. Even for basic on-off duty cycle control, the amount of energy applied to the windings and converted to mechanical energy can vary greatly with slight adjustments of switching angles. Current and flux-linkage profile tracking also relies heavily on accurate position feedback.

Sensorless SRM control methods estimate rotor position with sophisticated algorithms that usually monitor applied voltage and phase current to estimate flux linkage, which is ultimately used to determine position. While this approach is possible, it is usually best to avoid sensor-less control methods unless cost constraints or other considerations outweigh the amount of effort involved in developing accurate position estimation algorithms. Phase resistance is typically used in position estimation, and therefore, care must be taken to accommodate for variations of resistance with winding temperature. Accurate position estimation is more challenging with high-voltage systems, which incur higher amounts of electromagnetic interference (EMI) PWM chopping can present difficulty with voltage measurement, while EMI can affect voltage, current, and temperature measurements.

Angular position sensors typically fall into one of two categories: incremental and absolute. Incremental encoders provide incremental position information, and they sometimes include an indexing locator so absolute angular position can be determined once the index position is reached. However, start-up routines or sequences must be implemented to blindly jog the rotor until the index is reached. Absolute position sensors avoid this issue by providing accurate position information regardless of starting position, and therefore, they increase the certainty of proper controller operation. 
In general, approaches to obtain angular position include the following.

\section{Optical encoders}

Phototransistors are used to sense light, usually from an LED source, which is filtered by a multi-track encoder disk. Optical encoders typically provide absolute position, but inexpensive incremental varieties are available. Absolute optical encoders are highly susceptible to failure from shock or vibration, and they are difficult to integrate into a complex design since they usually connect to the end of a rotor shaft with a mechanical coupling.

\section{Hall-effect sensing}

Hall-effect sensors can be installed in a stator to determine rotor position by measuring magnetic fields. This approach is most common in permanent magnet motors but can be implemented in other types of electric motors, or used in stand-alone sensors. It can be difficult to obtain high accuracy with this approach, especially with large air gaps and machines without permanent magnets.

\section{Inductive pick-up}

Sensing coils located on a stationary frame can be used to count electrical transients received when exciting a rotating component that has magnetically salient teeth. This type of sensor is typically incremental, and an indexing locator can be integrated into the rotating component to facilitate absolute angular position measurement. If a second coil is staggered properly, the direction of rotation can be determined. Many types of methods use existing stator windings (sensor-less) or additional windings to estimate angular position. The most reliable and increasingly popular type of position sensor is the resolver, and therefore, a detailed explanation is given below.

\section{Resolver Background}

Position resolvers have sensor coils that determine absolute angular position, typically with sine and cosine sensor feedback. Some resolvers use permanent magnets (PMs) on the rotating component, and classic non-PM resolvers have coils on the rotor that are excited through brush contacts or through a brushless magnetic coupling and a second set of stator coils. Modern resolvers have excitation and sensor coils located on the same stator and a highly permeable rotor without permanent magnets or coils, but with a variable radius in order to facilitate a reluctance that varies with rotor position. Almost all hybrid and electric vehicle electric motors use resolvers for angular position feedback due to their low cost and capability to operate reliably in harsh environments. The second generation Toyota Prius (model years 2004 through 2009) motor resolver is shown on the left in Fig. 53. It has 14 stator teeth and 2 rotor poles, whereas the Hyundai Sonata hybrid motor resolver has 20 stator teeth and 8 rotor poles, as shown on the right in Fig. 53. The small Hyundai Sonata starter-generator resolver, shown in Fig. 54, has 12 stator poles and 3 rotor poles, and the Nissan Leaf motor resolver (Fig. 55) has 10 stator poles and 4 rotor poles.

Excitation windings are located on every tooth in these resolvers, and the polarity of each excitation coil alternates every tooth. Sensor coils are wound such that sine and cosine components are produced, and the excitation signal is modulated as the rotor rotates. Since the radius of the second generation Prius resolver rotor does not change abruptly, flux paths are mostly localized, as opposed to traversing through the entire rotor and around the back-iron of the stator. The excitation coils facilitate short flux paths amongst neighboring excitation coils because of their opposite polarities. 

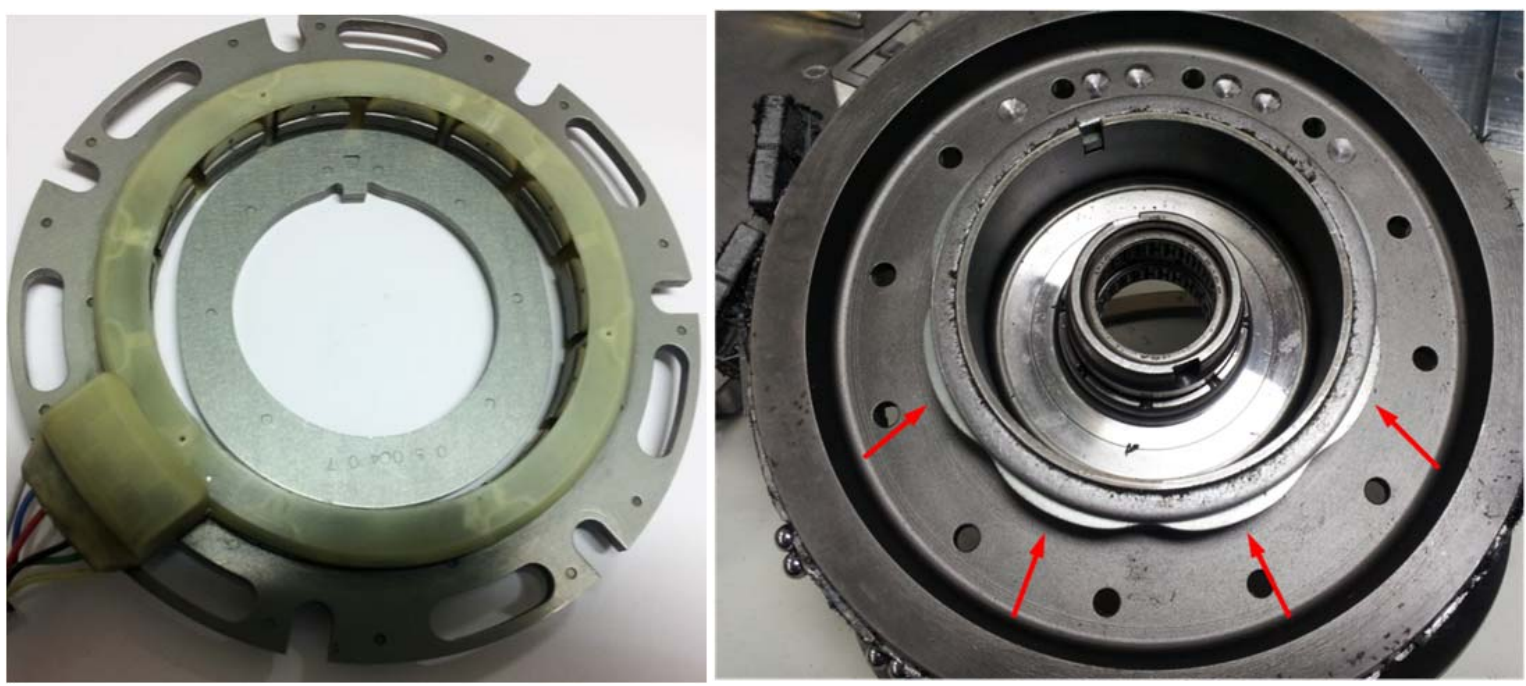

Fig. 53. Toyota Prius motor resolver (left) and Hyundai Sonata motor resolver (right).

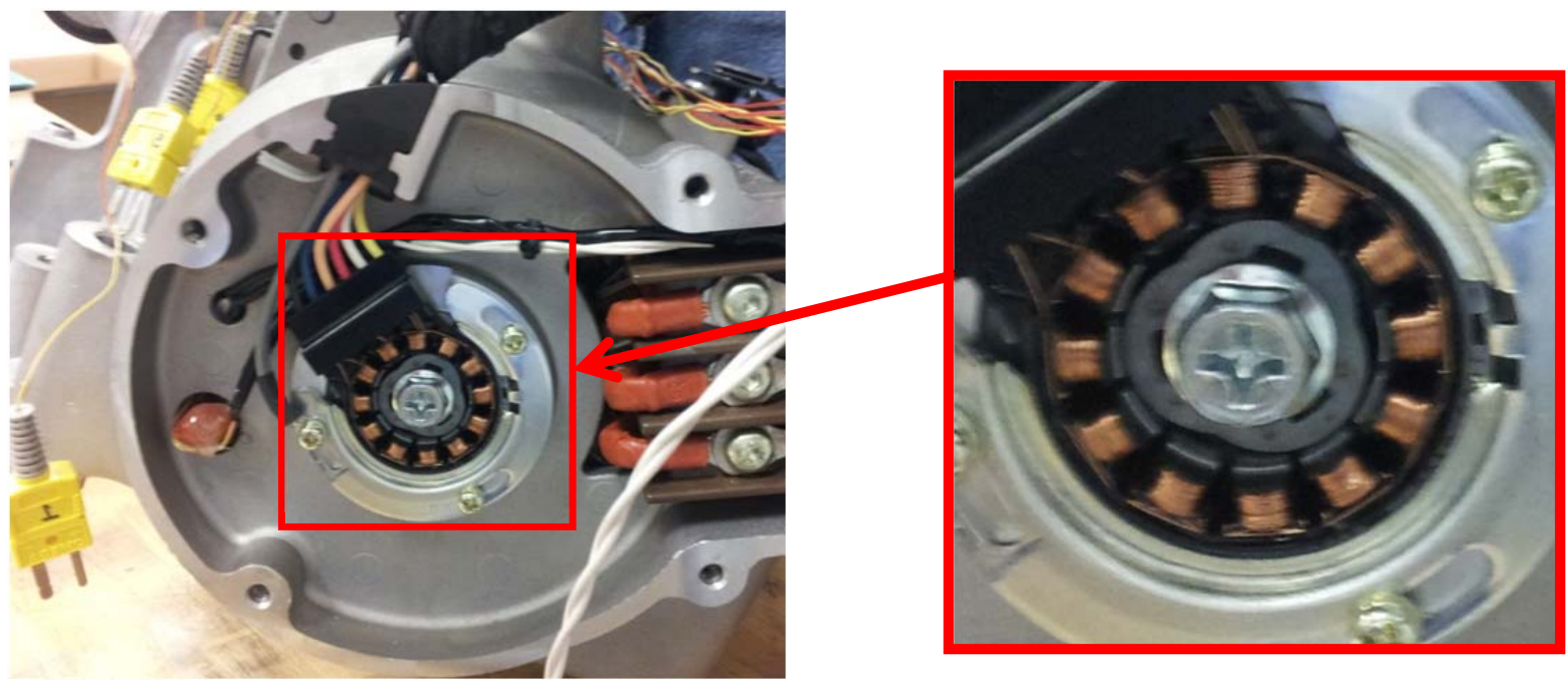

Fig. 54. Hyundai Sonata starter-generator resolver. 


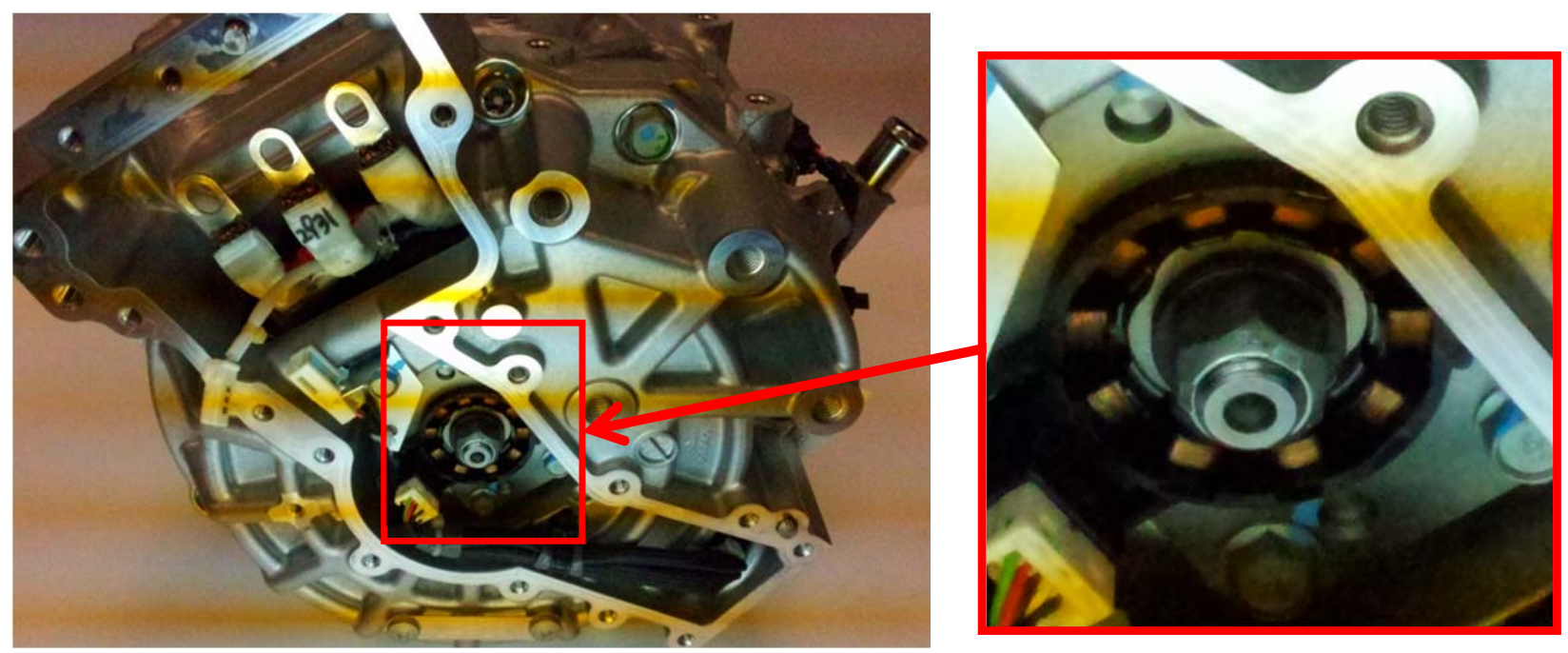

Fig. 55. Nissan Leaf motor resolver.

Typical resolver waveforms are shown in Fig. 56, with the excitation on the uppermost graph, sine/cosine signals on the middle graphs, and position in radians on the bottom graph. Excitation signals are sinusoidal, and they typically operate at a fixed frequency in the range of $5-20 \mathrm{kHz}$. Sine and cosine sensor signals are simply amplitude modulated at a frequency that is directly proportional to rotor speed. To demodulate the sensor signals, they can be multiplied with the excitation signal, which yields the desired fundamental signals $\left(+\mathrm{f}_{0}\right.$ and $\left.-\mathrm{f}_{0}\right)$ along with a 2 times excitation frequency $(10-40 \mathrm{kHz})$ component with fundamental frequency side bands $\left(2 \bullet f_{\text {exc }}+f_{0}\right.$ and $\left.2 \bullet f_{\text {exc }}-f_{0}\right)$ in the frequency spectrum. Therefore, the demodulated sine and cosine components (shown in red) can be obtained by applying a low pass filter to the output of the multiplier. While this operation can be easily implemented with analog circuitry or within a control algorithm, Tamagawa's AU6802 resolver converter integrated circuit (IC) is utilized in all of the automotive systems mentioned above. Analog device offers a similar IC called the AD2S. These ICs offer features such as noise immunity, reference regulation, buffering, and latching of the digital output. As with almost any type of absolute position sensing, it is important to latch and buffer the data correctly, such that all of the bits are in a well-defined state, as opposed to one or more bits being in a transient or floating state, which could cause significant control issues.

The resolver should be carefully located with respect to the motor since fringing of the magnetic field of the motor can affect the behavior of the resolver. This is especially important for switched reluctance motors, which often operate in high saturation and incur a significant amount of sharp electrical transients in comparison with sinusoidal machines like interior permanent magnet or induction machines. 

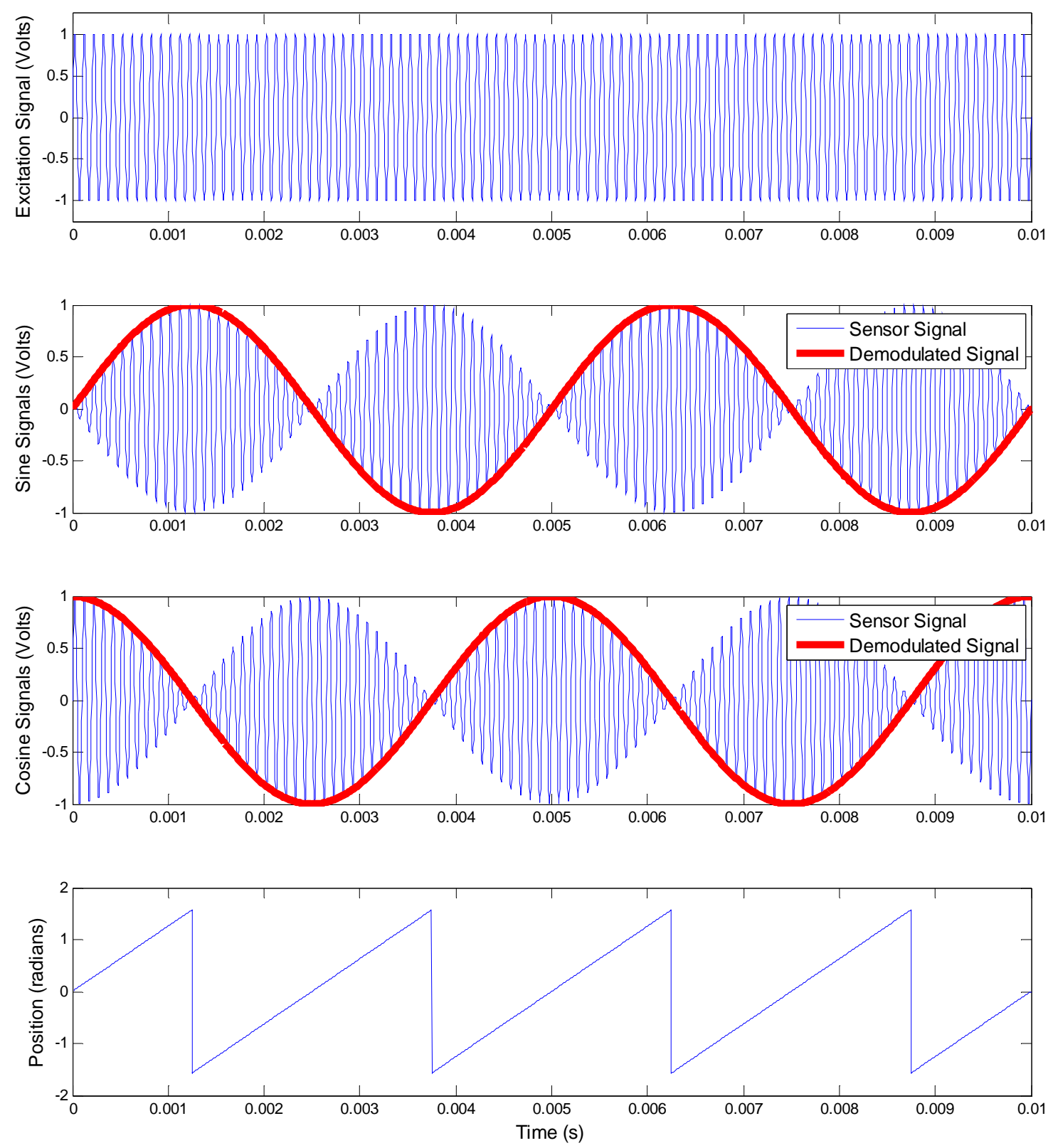

Fig. 56. Typical resolver signals.

\section{Resolver Model}

To facilitate the description of fundamental operations of modern angular position resolvers, simple resolver geometry is shown in Fig. 57. Commercially available resolvers include more stator poles for more continuous waveform behavior, but this simplified design is easier to understand. There are a total of 16 teeth with 8 teeth for cosine sensor coils (indicated with red) and 8 teeth for sine sensor coils (indicated with green). All teeth include excitation coils (indicated with blue), which are wound in a manner such that the flux path is localized since adjacent teeth are wound with opposite polarity. Notice how the air gap distance varies due to the oval shape of the rotor. Note that the cosine sensor coils located at the 3 o'clock and 9 o'clock positions have the same polarity as the excitation coils. Therefore, a voltage in phase with the excitation will be induced in these coils, and these could be called the positive cosine 
sensor coils. Contrarily, the cosine sensor coils at the 12 o'clock and 6 o'clock locations have opposite polarities with the excitation coils, and thus could be called the negative cosine sensor coils. Similarly, sine sensor coils at 1.5 o'clock and 7.5 o'clock could be called positive sine sensor coils, and sine sensor coils at 4.5 o'clock and 10.5 o'clock could be called negative sine sensor coils.

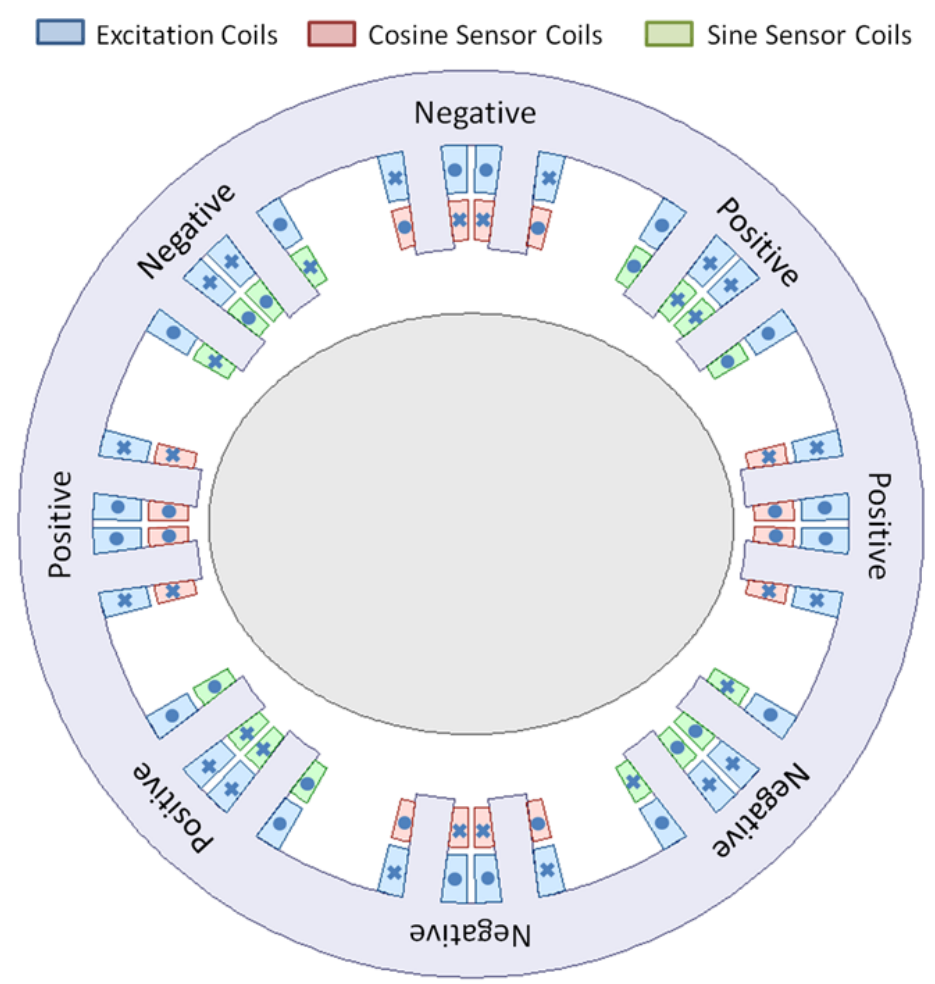

Fig. 57. Simplified angular position resolver geometry.

In Fig. 57, the resolver rotor is in its zero position, wherein the air gap between the rotor and the positive cosine sensors is minimized and the air gap between the rotor and the negative cosine sensor poles is maximized. Therefore, if all cosine sensor coils are connected in series, the maximum voltage induced in the cosine sensor coils occurs at this position. Note that the rotor is equidistant from both positive and negative sine sensor poles, and thus a series connection of all sine sensor coils causes positive and negative components to cancel and sum to zero. To summarize behavior, when the rotor is at a standstill in the zero position, maximum voltage is induced in the cosine sensor windings at the same frequency and phase as that of the excitation windings, whereas no voltage is induced in the sine sensor windings.

As the rotor rotates counterclockwise 45 mechanical degrees (90 electrical degrees), the air gap distance is minimized between the rotor and the positive sine sensor coils, and thus the maximum voltage amplitude is induced into the sine sensor coils at this position. Since the cosine sensor poles are equidistant from to rotor, the induced voltages in the cosine sensor coils sum to zero. As the rotor rotates clockwise to the 90 mechanical degree (180 electrical degree) position, the rotor poles are in alignment with the negative cosine sensor coils. Thus, a maximum voltage is induced into the cosine sensor coils at the same frequency of the excitation signal, but with opposite polarity, and thus a 180 electrical degree difference in electrical phase. Therefore, the demodulated output of the cosine sensor coils is at a minimum. The sine sensor coils produce no voltage at this position. Similarly, with the rotor positioned at the 135 mechanical degree position, maximum voltage is induced by the excitation signal, but it is 180 electrical degrees out phase, and the cosine voltage is zero. Rotating another 45 mechanical degrees to the 180 degree location causes the rotor poles to be in alignment with the positive cosine sensor poles, and 
the magnetic circuit is the same as it is when the rotor at 0 degrees. Therefore, a full electrical cycle is completed in half of a mechanical cycle.

The behavior of the resolver when the rotor position is between the angular positions described above depends largely on the shape of the rotor and the number of turns in the excitation and sensor windings. Development of a basic resolver model provides a better understanding of resolver operation, and a proficient means to study the impact of various resolver rotor shapes. A complete design process should include characterization of the inductance profiles of the coils versus position with FEA software along with refinements from empirical data. A basic model will be developed with consideration of excitation coils, sensor coils, and reluctance components shown in Fig. 58. It can be assumed that stator core, stator tooth, and rotor core reluctances are constant. However, the reluctance of the air gap varies with the nonuniform radius of the resolver rotor. The reluctances of the air gaps associated with the positive cosine excitation and sensor coils shown in Fig. 58 can be designated as $R_{g a p_{-} c p 1}$ and $R_{\text {gap } \_p 2}$. Designations for all gap reluctances are shown in Fig. 59. Nomenclature and subscripts for magnetic fluxes, inductances, and induced voltages will be defined in a similar manner. Assuming no coupling with other coils, no fringing or leakage, and a series connection of the two excitation coils, the amount of magnetic flux in the excitation coils shown in Fig. 65 is the same. More details are available in Appendix A.

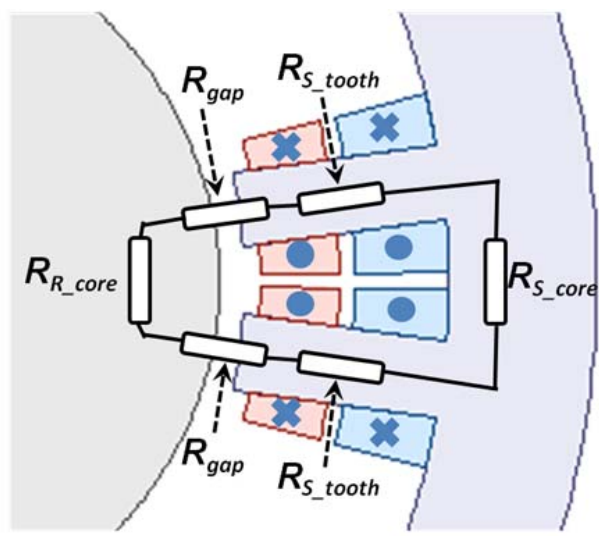

Fig. 58. Reluctance components for a basic resolver model. 


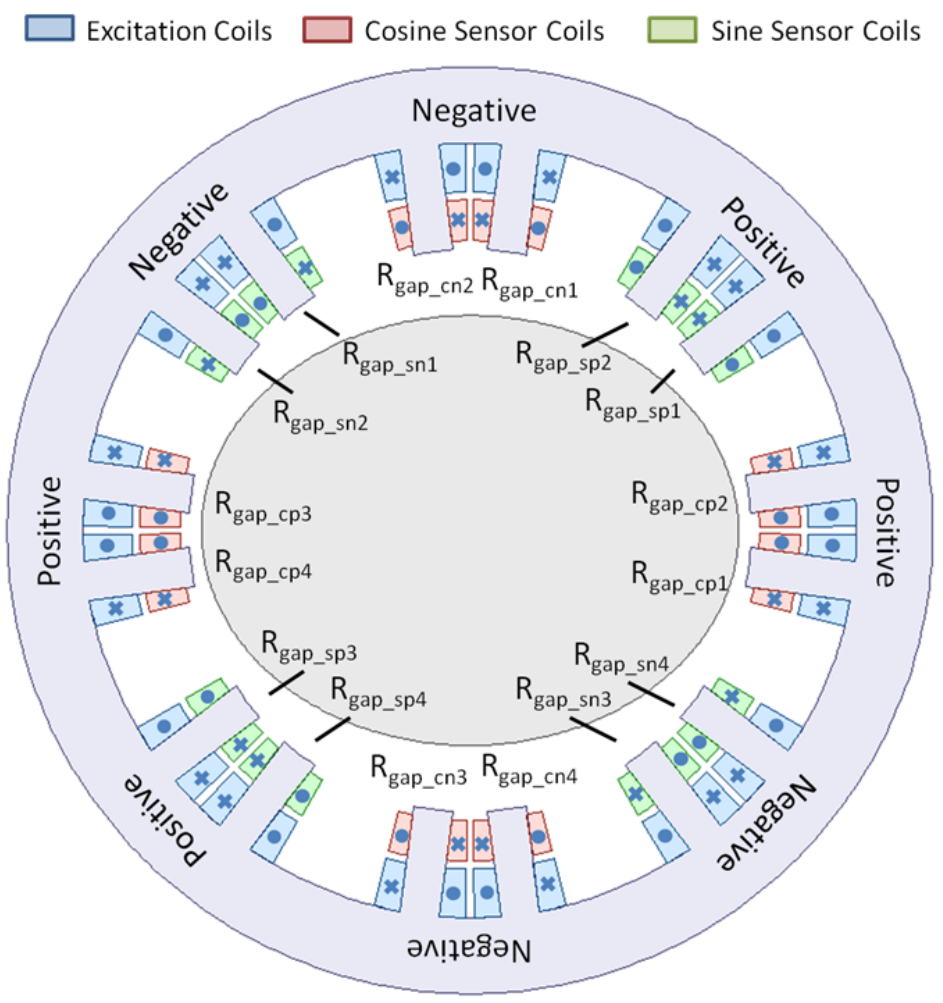

Fig. 59. Gap reluctance designations.

Reluctance can be generally defined by

$$
R=\frac{l}{\mu_{0} \mu_{r} A},
$$

where

$l$ is the length of the magnetic path, $\mu_{0}$ is the permeability of free space $\left(4 \pi \cdot 10^{-7}\right.$ Henries $\left./ \mathrm{m}\right)$, $\mu_{r}$ is relative permeability of the material ( $\sim 1$ for air and 8000 for electrical steel), and $A$ is the cross-sectional area of the magnetic path.

Using dimensions similar to that of the 6-4 SRM described in Section 5.5.1, the following parameters are approximated:

$$
\begin{aligned}
& l_{S_{-} \text {tooth }}=0.025 \mathrm{~m} \\
& l_{R_{-} \text {core }}=0.0165 \mathrm{~m} \\
& l_{S_{-} \text {core }}=0.027 \mathrm{~m} \\
& A=0.00924 * 0.004=3.6960 \mathrm{e}-005 \mathrm{~m}^{2}
\end{aligned}
$$

For illustrative purposes, consider an elliptical rotor with a radius defined by

$$
r_{\text {rotor }}(\delta)=r_{\text {rotor_max }} \sqrt{\cos (\delta)^{2}+\left(K_{\text {ellipse }} \sin (\delta)\right)^{2}}
$$


where $K_{\text {ellipse }}$ is a scalar between zero and 1 , and $\delta$ is an angle which varies from zero to $2 \pi$ as the radius of the rotor is defined. Therefore, as shown in Fig. 60, the angle, $\delta$, has a reference that rotates with the rotor angle, $\theta$. The length of the air gap at each tooth can be described by

$$
l_{\text {gap }}(\theta)=r_{\text {Stator_in }}-r_{\text {rotor }}\left(\delta=\theta_{\text {tooth }}-\theta\right) \text {, }
$$

where $r_{\text {Stator in }}$ is the inner radius of the resolver stator $(66.5 \mathrm{~mm})$ and $\theta_{\text {tooth }}$ is the static angular location of each tooth. To consider the impact of magnetic gap size upon the reluctances mentioned above, values of $\theta_{\text {tooth }}$ for the air gap lengths, $l_{\text {gap_cp }}, l_{\text {gap_cp }}, l_{\text {gap_cp } 3}, l_{\text {gap } \_c p 4}, l_{\text {gap_cn }}, l_{\text {gap_cn }}, l_{\text {gap_cn } 3}, l_{\text {gap_cn } 4}, l_{\text {gap_sp1 }}, l_{\text {gap_sp } 2}$, $l_{\text {gap_sp3 }}, l_{\text {gap_sp } 4}, l_{\text {gap_sn }}, l_{\text {gap_sn } 2}, l_{\text {gap_sn } 3}$, and $l_{\text {gap_sn } 4}$, are $-7.5,7.5,172.5,187.5,82.5,97.5,262.5,277.5,37.5$, $52.5,217.5,232.5,127.5,142.5,307.5$, and 322.5 degrees, respectively. These gap lengths can be used to determine gap reluctances $R_{\text {gap_cpl }}, R_{\text {gap_cp } 2}, R_{\text {gap_cp } 3}, R_{\text {gap_cp } 4}, R_{g a p \_c n 1}, R_{\text {gap_cn } 2}, R_{\text {gap_cn } 3}, R_{\text {gap_cn } 4}, R_{\text {gap_spl }}$, $R_{\text {gap_sp } 2}, R_{\text {gap_sp } 3}, R_{\text {gap_sp } 4}, R_{\text {gap_sn } 1}, R_{\text {gap_sn } 2}, R_{\text {gap_sn } 3}$, and $R_{\text {gap_sn }}$. Substitution of the reluctances into inductance equations ultimately yields the total inductance of the excitation circuit, $L_{\text {exc_total }}$. With an excitation voltage expressed by

$$
V_{e x c}=A_{e x c} \sin \left(w_{e x c} t\right) .
$$

The excitation current can be determined by

$$
i_{\text {exc }}=\frac{V_{\text {exc }}}{R_{\text {exc }}+L_{\text {exc_total }}},
$$

where $R_{\text {exc }}$ is the combined resistance of all excitation windings. Using the equations above, magnetic flux and induced voltage can be calculated for each sensor coil. As previously described, Fig. 56 shows ideal normalized excitation, sensor, demodulated sensor, and position waveforms. After the signals are demodulated, the rotor angle can be found by calculating the inverse tangent of the sensed sine and cosine components:

$$
\theta_{\text {measured }}=\tan ^{-1}\left(\frac{\varepsilon_{\text {sen_s_total }}}{\varepsilon_{\text {sen_c_total }}}\right) \text {. }
$$

Although a simple ellipse equation was chosen to represent the rotor radius for illustrative purposes, demodulated sine and cosine components are relatively close to ideal waveforms. The influence of the ellipse factor, $K_{\text {ellipse }}$, is illustrated in Fig. 61, where ideal demodulated sine waveforms are shown in comparison with modeled demodulated sine waveforms for $K_{\text {ellipse }}$ values of 0.9, 0.95, and 0.97. Angular position error is shown in Fig. 62, and as $K_{\text {ellipse }}$ increases, the amount of error decreases. However, some magnetic saliency is needed to derive sine and cosine components, and a factor of unity, which correlates with a circular rotor, is not a feasible option. Note that even with a basic definition of resolver rotor radius, angular position error is less than 1 percent with a factor of 0.97 . The ratio of the minimum radius at $\delta=90$ degrees and maximum radius at $\delta=0$ of the second-generation Prius resolver shown in Fig. 53 is 0.926 . Though close, the Prius resolver radius is not matched when using the ellipse equation and a factor value of 0.926 . In application, usually more stator poles are used for sensor windings, and the windings have a semi-sinusoidal distribution of turns around the pole. 


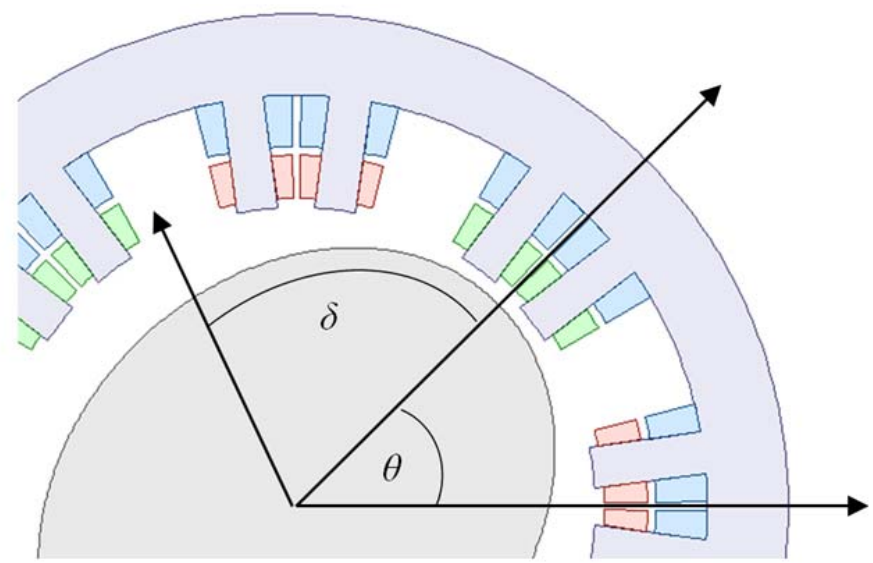

Fig. 60. Angle definitions.
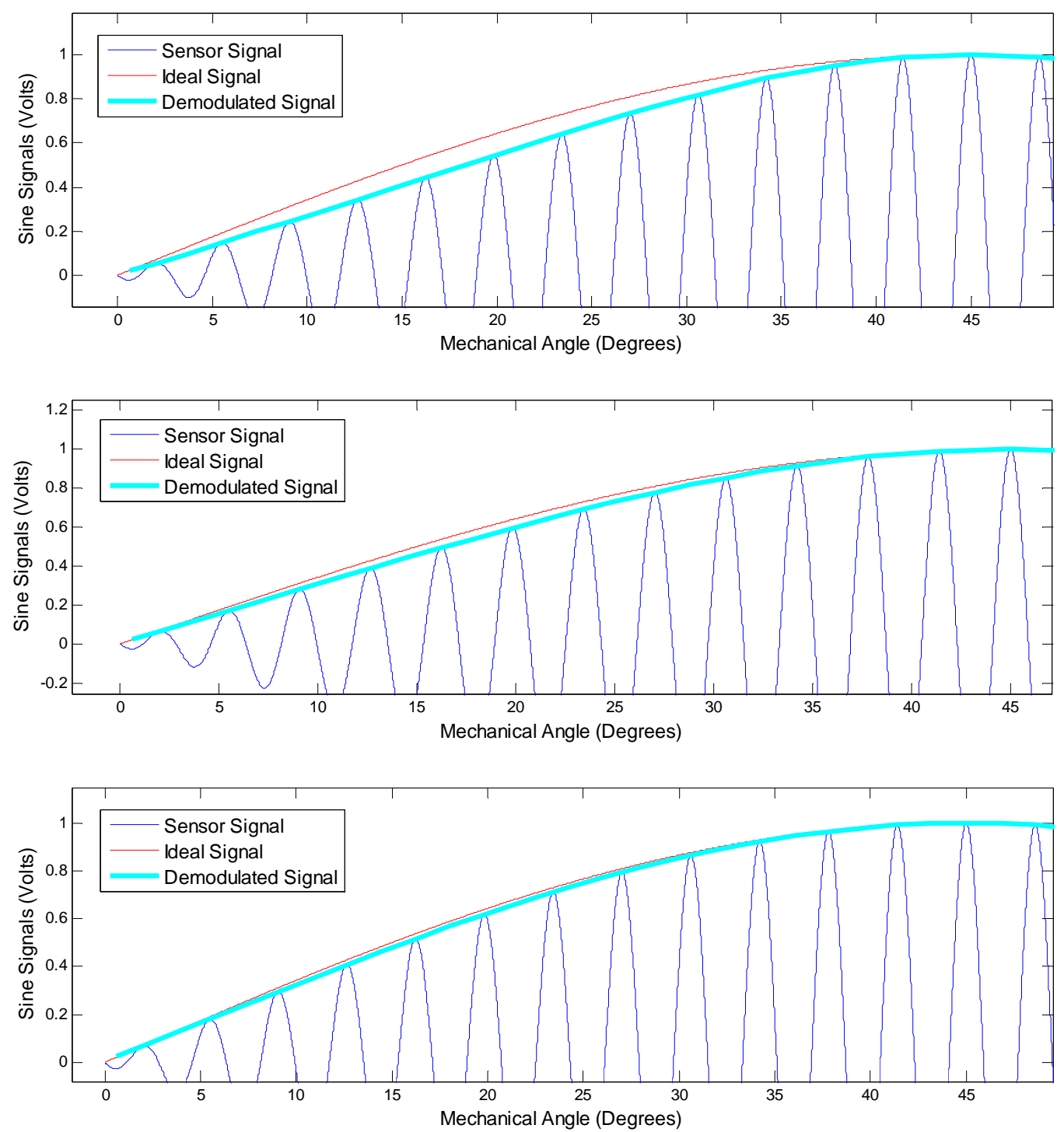

Fig. 61. Sine signals with $K_{\text {ellipse }}$ values of 0.9 (top), 0.95 (middle), and 0.97 (bottom). 

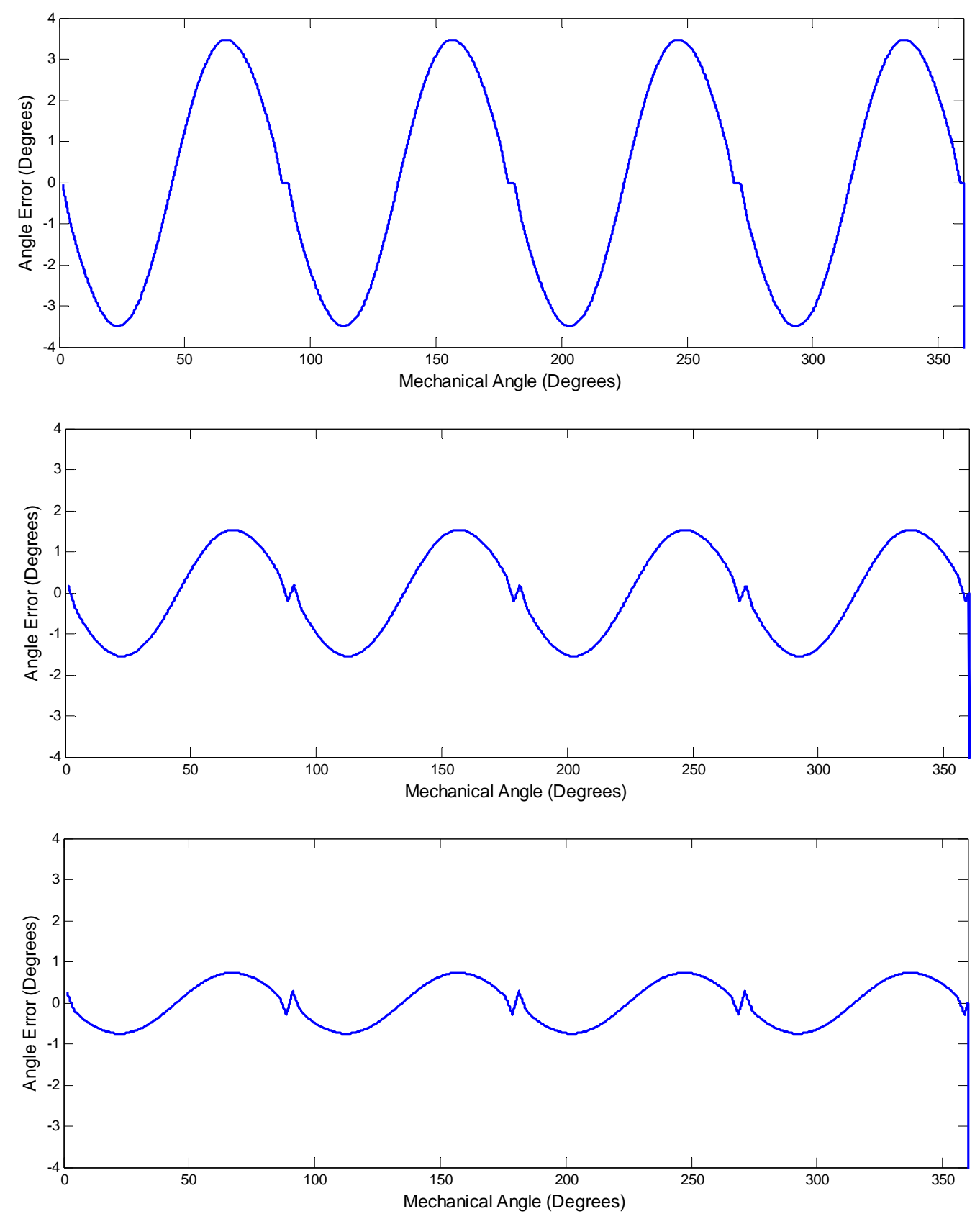

Fig. 62. Angular measurement error with Kellipse values of 0.9 (top), 0.95 (middle), and 0.97 (bottom).

\subsection{HIGH-TEMPERATURE WIRING}

Typical motor and sensor wiring is composed of copper or copper alloy and coated with a thin electrical insulation layer intended for operating temperatures well below the environment of the canner rotor pump. Typical varnish insulation (e.g., Formvar ${ }^{\mathbb{B}}$ ) has operational limits of less than $105^{\circ} \mathrm{C}$. For hightemperature applications in motors and transformers, polyimide insulated magnet wire is capable of operation up to $250^{\circ} \mathrm{C}$. Completed coils are frequently vacuum impregnated with an insulating varnish to improve insulation strength, inhibit humidity influx, and ensure long-term reliability. Achieving operating temperatures to $700^{\circ} \mathrm{C}$ requires a non-organic, ceramic, or glass insulation type. Ceramic insulation imposes additional constraints on the conductor material so that the ceramic adheres to the wire during the 
stages of curing. Additionally, higher temperatures drive ion migration, and therefore, insulator-conductor compatibility becomes a concern. A detailed discussion of the properties of the relevant materials is in Appendix A.

\subsection{BARRIER CANS}

Two protective and separating metal cans are used in this motor design: one can to protect the magnetic materials in the rotor and the other can to act as a barrier between liquid salt and the local environment. (See Fig. 63 and Fig. 64 for details of cans orientation.) Issues related to the cans are mostly material selection. The innermost can is attached to the outside of rotor to protect the magnet laminations from contact with the liquid salt. The can rotates with the rotor as a unit. The magnet rotor material is an alloy of iron and cobalt that is easily corroded by liquid salt. The outer can fits between the rotor and stator components and does not rotate. Likely, both cans will be constructed of the same alloy. Selection of material is based on corrosion, magnetic penetration, and strength.

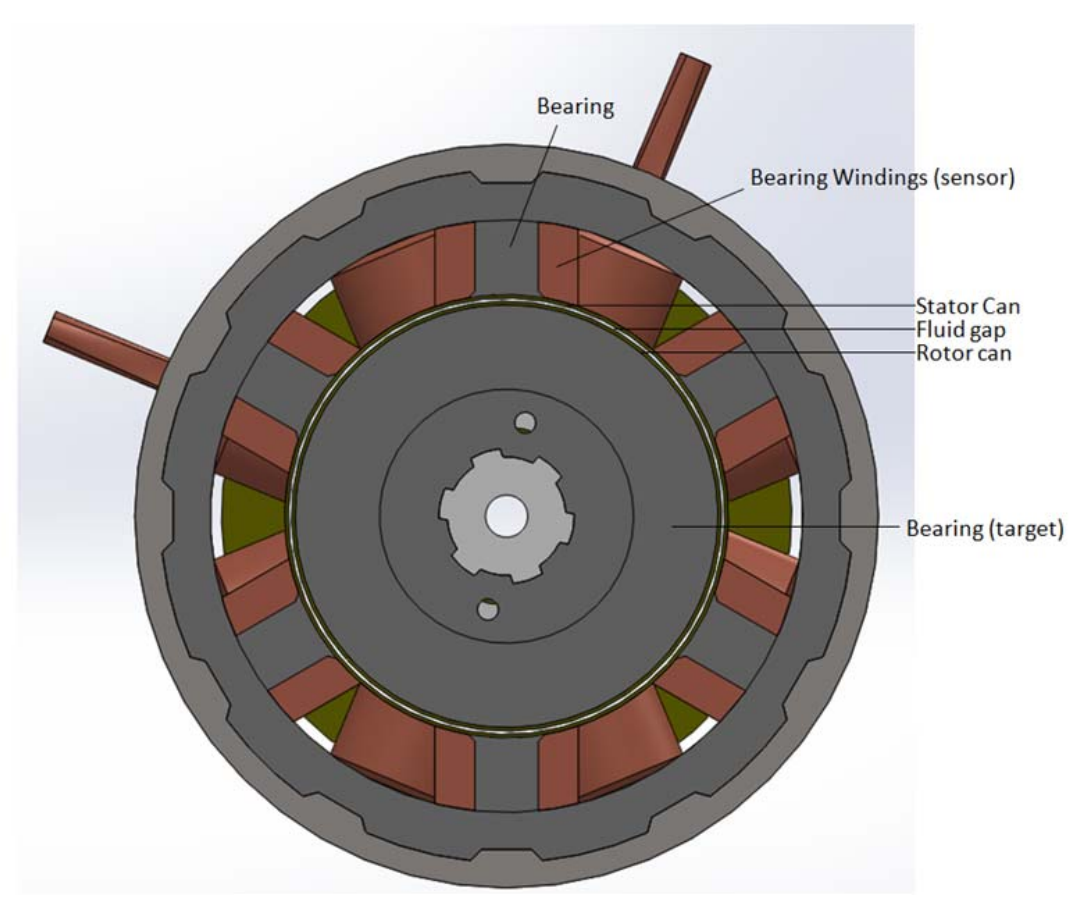

Fig. 63. Cross section of motor bearing showing gap location. 


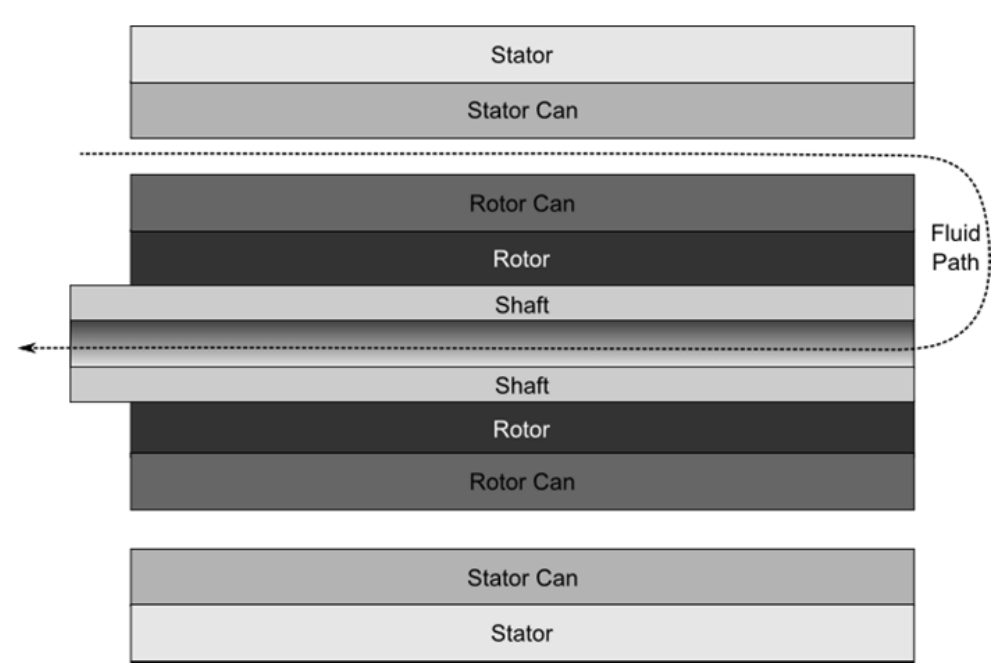

Fig. 64. Illustration of materials stack in motor (not to scale).

\subsubsection{Corrosion}

The stator can and the rotor can protect the rotor, stator, bearings, and other motor components from interaction with the FLiNaK fluid. For this reason, the material chosen for the stator-can and rotor-can must be corrosion resistant to the molten salt. Such materials are available and are presented in Appendix A.

\subsubsection{Magnetic Penetration}

The cans that will be surrounding the rotor and stator assemblies, protecting the components from coolant penetration, must allow adequate magnetic field penetration. Eddy currents will be induced in the surface of the can in response to the ac magnetic fields applied by the stator, limiting the rate at which the stator magnetic fields can diffuse through the thin-walled can material, and resulting in power dissipation. The penetration time of the magnetic field corresponds to the decay time of the induced eddy currents, which is determined by the electrical conductivity of the can, the thickness of the can, and the area of the stator pole faces. The characteristic $-3 \mathrm{~dB}$ frequency response of the can, as shown in Figs. 65 and 66, is estimated based on the e-folding L/R decay time of the eddy currents. The parameter w/D shown in the plots is the assumed aspect ratio for the circulating eddy currents, where $\mathrm{D}$ is the effective diameter of a stator pole face and $\mathrm{w}$ is the width of the eddy current channel. For the three candidate materials shown in the plots, the shift in the frequency response between $20^{\circ} \mathrm{C}$ to $800^{\circ} \mathrm{C}$ is only that due to the change in electrical resistivity with temperature (i.e., the magnetic permeability of nickel at $20^{\circ} \mathrm{C}$ is not included). Hastelloy N provides the most transparent can and features a transparency that does not change significantly with temperature. In that case, the eddy current power losses at room temperature and at operating temperature will be very similar. For acceptable eddy current power loss, the operating frequency must be well below the characteristic frequency, preferably by a factor of 5-10. It might also be noted that a factor of 5 provides $99.3 \%$ magnetic field penetration (five e-folding times). Below the characteristic frequency, the power dissipation associated with the eddy current ohmic losses increases as frequency squared and would be quite large at frequencies approaching the value of the characteristic frequency shown in the plots. Depending on the can material, a wall thickness of $1 \mathrm{~mm}$ or less may provide both reasonable magnetic penetration and sufficiently low eddy current loss for operating frequencies below $10 \mathrm{kHz}$. 


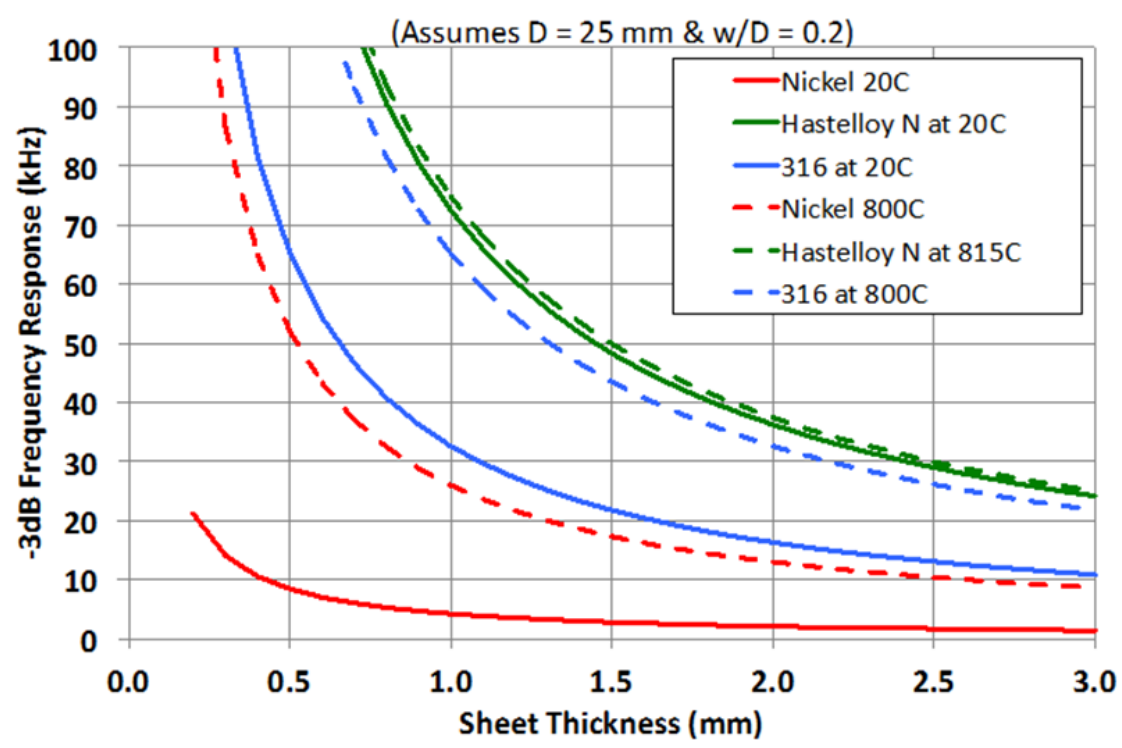

Fig. 65. Characteristic frequency response as a function of thickness.

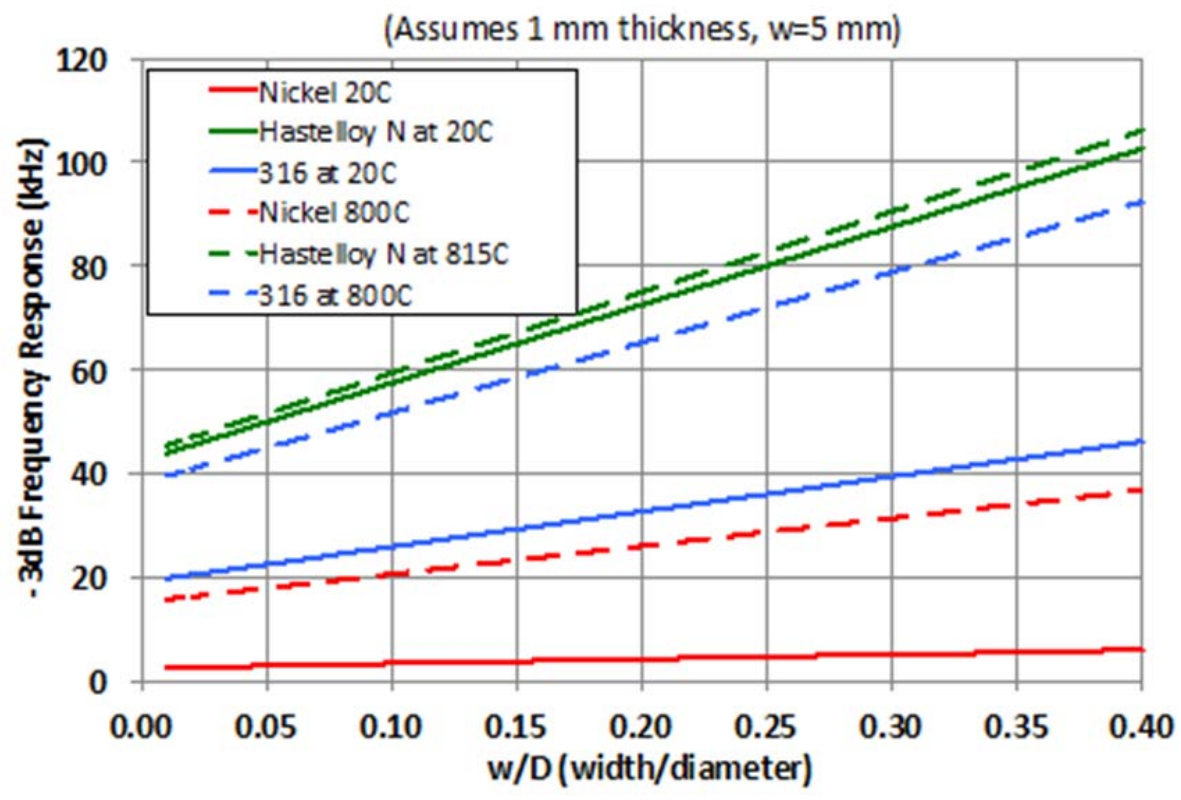

Fig. 66. Characteristic frequency response as a function of aspect ratio.

\subsubsection{Smoothness}

It is necessary that the determined fluid gap between the rotor and stator cans be maintained to avoid touch-down and to ensure proper motor operation. Additionally, it is important that the fluid flow is consistent through the flow path to achieve adequate motor cooling. For this reason, the desired surface finish of the cans must be determined. Several methods are available for smoothing the surface including electro-polishing and post-machining. 


\subsection{MAGNETIC BEARINGS}

The high operating temperature and coolant corrosion compatibility require that the rotating parts of the motor pump be suspended without material contact. The only viable solution is suspension by magnetic bearings. The magnetic bearings must provide adequate steady-state forces for the steady-state shaft loads discussed in previous section. Additionally, the magnetic bearings must provide adequate transient response for pump disturbance forces noted in Table 9. Bearing locations and motor cross section are shown in Figs. 67 and 68. The motor coordinate reference is shown in Fig. 69.

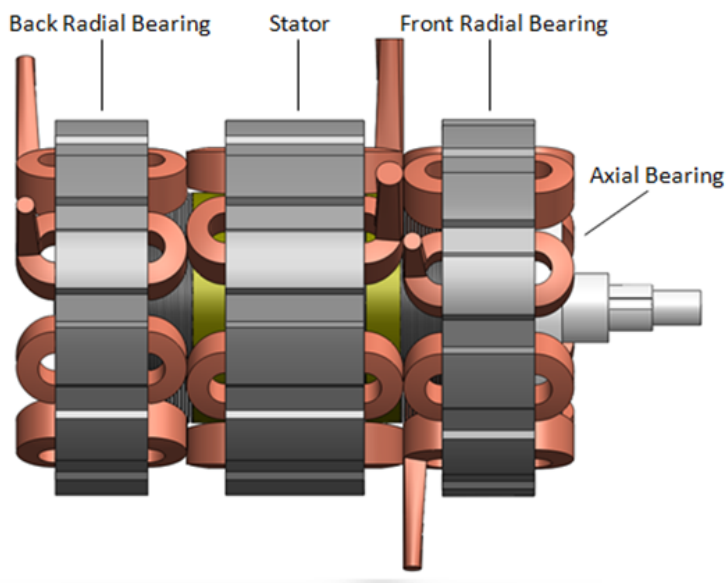

Fig. 67. 3D rendering of motor concept showing axial and radial bearings and main torque drive section.

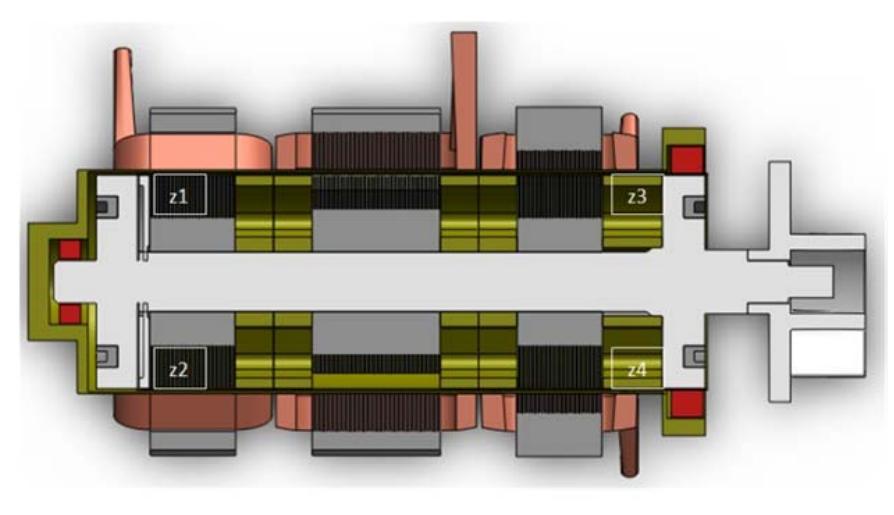

Fig. 68. Motor-pump cross section.
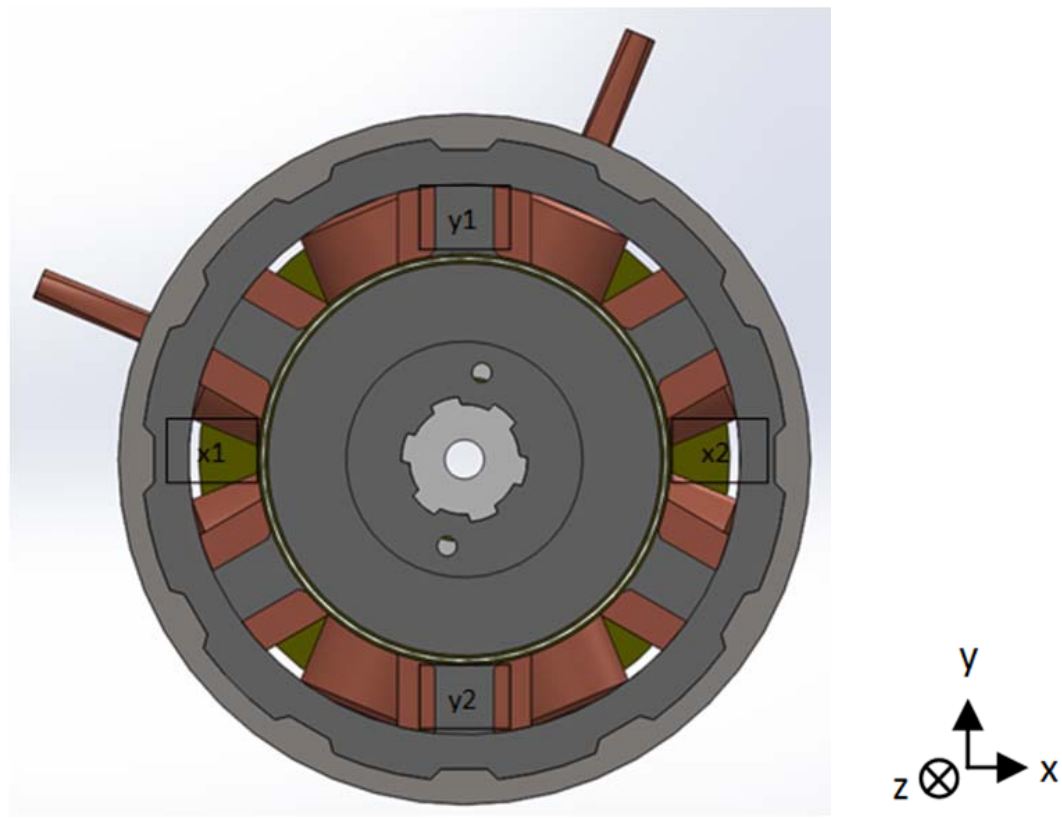

Fig. 69. Motor coordinate orientation. 


\subsection{ENVIRONMENTAL CONDITIONS}

\subsubsection{Pumping Fluid Composition}

For the purposes of this demonstration, $\operatorname{FLiNaK}(46.5,11.5$, and 42 mole percent, respectively) will be employed. In the actual reactor, $\mathrm{FLiBe}$ [ $\mathrm{LiF}-\mathrm{BeF}_{2}$ (67 and 33 mole percent); melting point of $\left.459^{\circ} \mathrm{C}\right]$ will be employed. FLiNaK is used a surrogate for FLiBe due to the toxicity of beryllium. The thermophysical properties of FLiNaK are available in Reference 47.

\subsubsection{Fluid Temperature, Density, and Viscosity}

The melting point of FLiNaK is $454^{\circ} \mathrm{C}$, and it will be used operating as a heat transfer fluid in the ORNL test loop. Many chemical properties of the fluid vary as a function of temperature. Experimental data show that density and viscosity are temperature dependent, and the following correlations have been derived:

$$
\rho=2.53-0.00073 T
$$

where $\rho$ refers to the fluid density in $\mathrm{g} / \mathrm{cm}^{3}$.

$$
\mu=0.04 e^{4170 / T}
$$

where $\mu$ refers to the fluid viscosity in Pa-s.

Two representative correlations are given in Fig. 70.

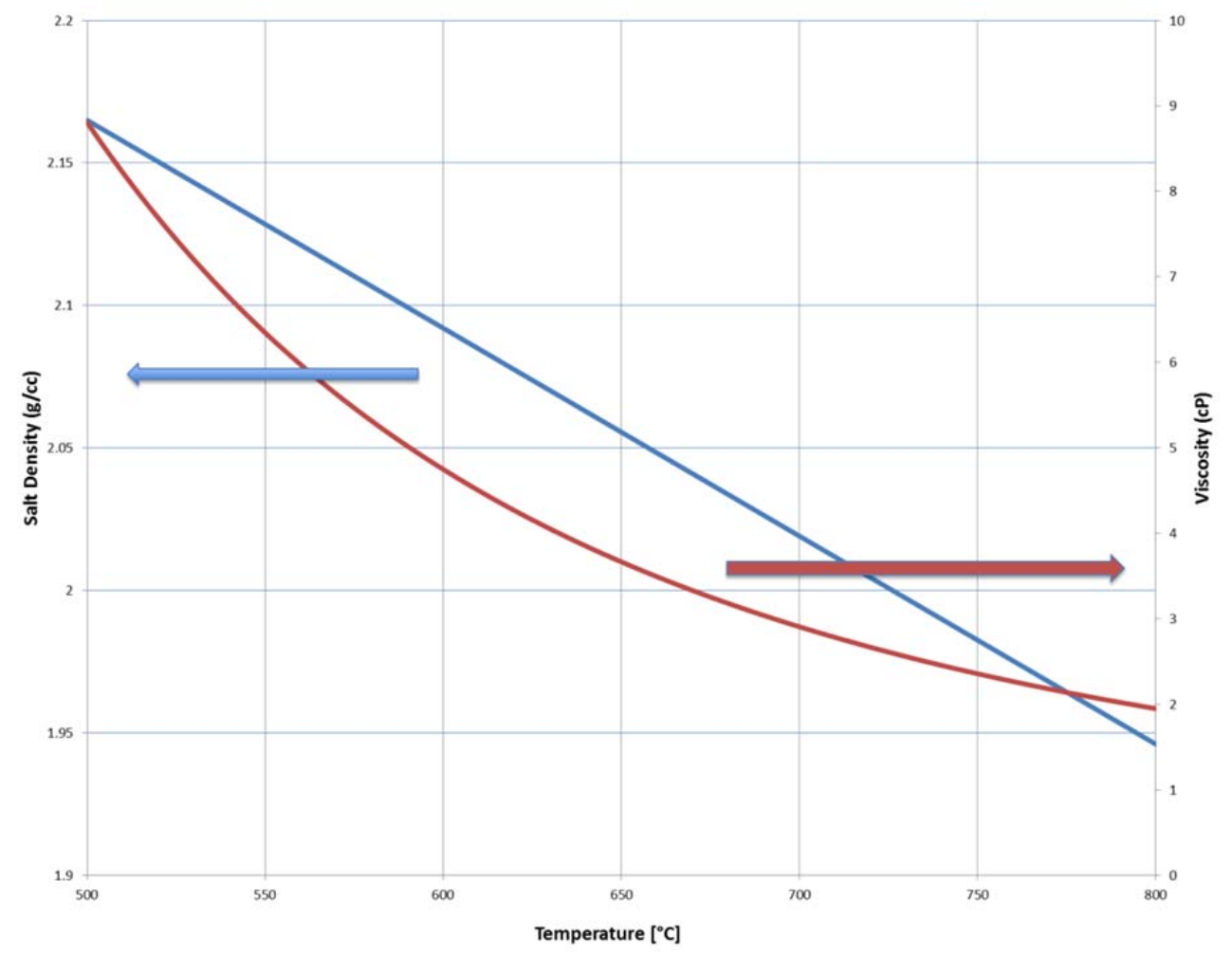

Fig. 70. Density-viscosity correlations for salt mixture. 


\subsubsection{EMI/RFI/Acoustics}

Specific requirements for both EMI and RF susceptibility and emissions are not currently available. However, it is reasonable to follow typical industrial practices to minimize emissions and susceptibility such as using shielded cables for the motor PWM leads and shielded cables for the sensor and instrumentation wiring.

The motor acoustic emissions should be estimated and verified during prototype testing. The acoustic emissions should not exceed typical industrial limits and must not provide detrimental effects to the cooling system piping and structure. Reluctance drive motors typically generate high-levels of vibration.

\subsection{RELIABILITY}

System-level reliability is determined by combining the reliability of the sub-systems for structural failures, functional failures, and other categories of failures. Various established statistical techniques can be utilized for quantifying the individual failure modes and the associated interactions at a system level. Markov transition diagrams can be used to quantify the independence and dependence of events, the standby redundancies, the common cause vulnerabilities, components supporting loads, and the mutually exclusive primary events. ${ }^{48}$

The system-level reliability goal is described in Section 5.11.5. The FMEA discussed in Section 3.4 provides the foundational data for the reliability assessment. The reliability of the various sub-systems is described as meeting or failing the reliability goal for each sub-system. The sub-systems with failing reliability require improvements by one or more of the following approaches.

1. Modify the system design to remove the failure mode stimulus.

2. Improve the reliability with a design improvement.

3. Combine self-testing, monitoring, and other techniques to detect and diagnose the failure and then provide an appropriate mitigation action to avoid the system effect of the failure event. ${ }^{49,50}$

4. Design redundancy as required to avoid the system effect of the failure event.

5. Perform prognostic functions to identify future failure events proactively.

These improvements will either reduce the failure probability or avoid the system effect of the failure as shown in Fig. 71. The net result is suitable system-level reliability despite various levels of sub-system reliability. Sections 5.11.1, 5.11.2, 5.11.3, 5.11.4, and 5.11.5 continue to describe a system concept for meeting the desired reliability. 


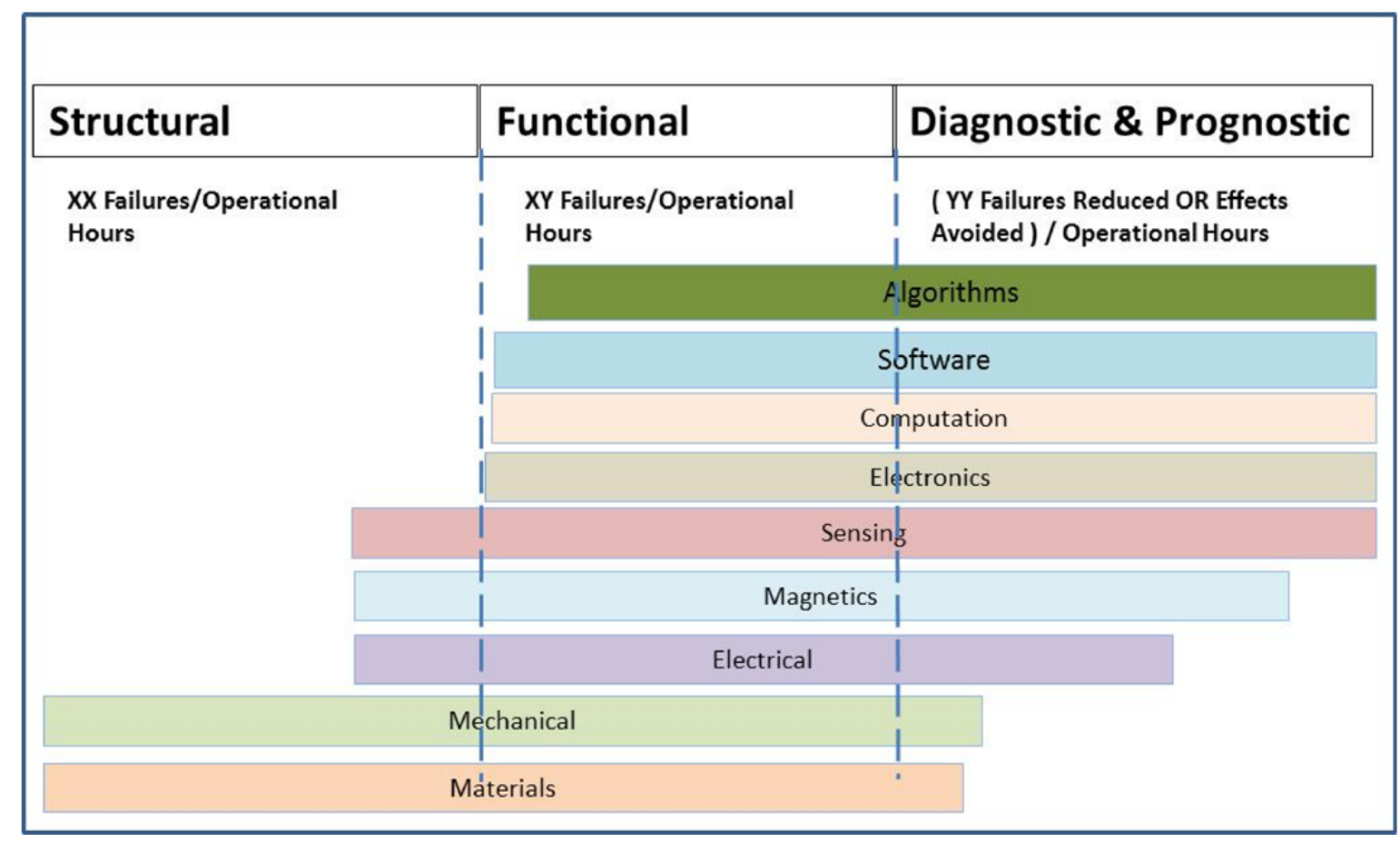

Fig. 71. System-level reliability composite.

\subsubsection{Graceful Degradation and Single Point Failure}

It is desired that a single failure in the canned rotor pump does not propagate to other motor components and does not affect the reactor system as a whole. For this reason, redundancy can be added in order to have alternative means of achieving a system function.

Graceful degradation, or fault tolerance, is expected from the canned rotor pump. This requirement implies that the pump will continue normal operation despite a given system failure. For this purpose, failure avoidance, detection, and remediation will be put in place.

Some typical faults, along with respective avoidance or reactionary strategy, are given in Table 13. 
Table 13. Fault summary

\begin{tabular}{ll}
\hline \multicolumn{1}{c}{ Fault } & \multicolumn{1}{c}{ Reaction } \\
\hline $\begin{array}{l}\text { Short Power electronics or amplifier fault } \\
\text { (such as current overload) }\end{array}$ & Anternal \\
Power electronics or amplifier failure & Component redundancy with reconfigurable amplifiers \\
Position sensor fault & Run-out compensation or appropriate filtering \\
Position sensor failure & Sensor redundancy \\
Loss of I/O board channel & Component redundancy \\
Computer Hardware failure & Back-up hardware \\
Software errors & Robust control algorithms \\
& External \\
Changes in dynamic rotor loading & Appropriate bearing control and appropriate touch-down \\
& strategy \\
Abnormal base motion & High stiffness bearings \\
& Control system that achieves acceptable comproise on \\
& bearing stiffness and damping requirements \\
Internal/External \\
Control system compensation \\
Touch-down bearings \\
Cracked rotor & Magnetic bearing compensation \\
Rotor contact & Touch-down bearings \\
& Appropriate control system for recovery after contact \\
& Alternative power supply \\
\hline
\end{tabular}

Refer to Section 3.4 for more information on failure modes, failure detection, and related control strategy.

\subsubsection{Diagnostics}

The operation of the canned-rotor pump should be monitored and logged throughout the pump lifetime in order to perform maintenance at the required intervals, as well as to record faults and to predict time to failure. The duration and amount of occurrences of start-up, shutdown, and steady-state operation should be recorded. The time history of other parameters, such as current, voltage, bearing forces, temperature, etc., should be recorded as well.

\subsubsection{Embedded and Intrinsic Sensors}

In order to detect system faults and failures and perform the necessary response steps, several measurements are necessary. The following sensors are required for failure detection.

- Temperature sensors

- Vibration sensors

- Position sensors

- Speed sensors 
- Flow meter

- Current monitoring

- Voltage monitoring

\subsubsection{Self-Testing and Monitoring}

A series of routine automated tests and monitoring should be performed during power-up of the system, after power-up and before pumping, and during normal operation. The first set of tests will be the PowerOn Built-in Test (PBIT), which will be performed during the powering of the system and will check the status of the necessary components to run the system. Once the test is performed, the pump should be heated to achieve proper fluid state, and a series of tests should be performed during the Initial Built-in Test (IBIT) to ensure proper operation of the pump. Once the IBIT is performed, normal operation can be started, and the Continuous Built-in Test (CBIT) may proceed, which monitors the proper functioning of the system. Figure 72 illustrates each test regime and operating condition in their respective order. Tables 14, 15, and 16 show details of each test to be performed.

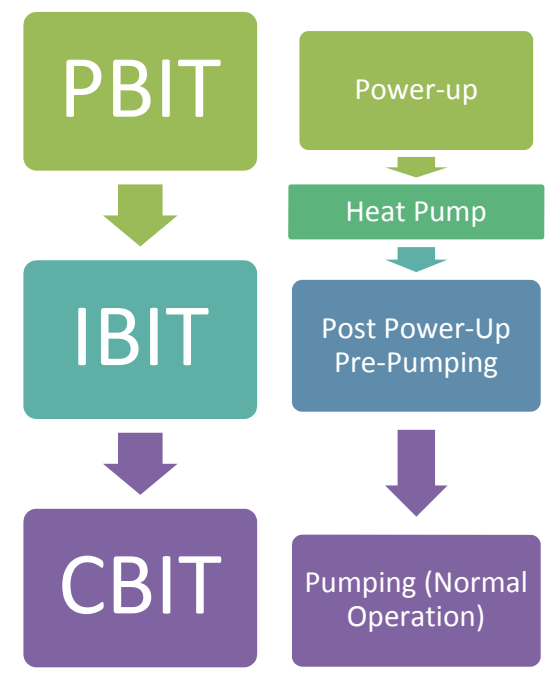

Fig. 72. Test regime and operating condition.

Table 14. Power-on Built-in Test

\begin{tabular}{ll}
\hline \multicolumn{1}{c}{ Test } & \multicolumn{1}{c}{ Purpose } \\
\hline Processing Memory check & Memory hardware failure check \\
Power supply current/voltage check & $\begin{array}{l}\text { Confirm proper functioning of electrical supply, power } \\
\text { supply, and power electronics }\end{array}$ \\
Sensor check & $\begin{array}{l}\text { Confirm proper operation of sensors and measurement } \\
\text { equipment }\end{array}$ \\
\hline
\end{tabular}


Table 15. Initial Built-in Test

\begin{tabular}{ll}
\hline \multicolumn{1}{c}{ Test } & \multicolumn{1}{c}{ Purpose } \\
\hline $\begin{array}{l}\text { Controlled X-Y-Z displacement of rotor. } \\
\text { Measurement of displacement }\end{array}$ & $\begin{array}{l}\text { Ensure proper operation of magnetic bearings. Identification of any } \\
\text { unexpected suspended solids in the fluid } \\
\text { Test proper operation of displacement sensors }\end{array}$ \\
$\begin{array}{ll}\text { Rotation of stator to specific speed } \\
\text { Angular velocity measurements }\end{array}$ & $\begin{array}{l}\text { Ensure proper operation of stator and rotor } \\
\text { Identification of rotating unbalance }\end{array}$ \\
Position measurements & Test proper operation of angular displacement sensors \\
Rotation of stator to specific speeds & Ensure proper pump operation \\
Pressure measurements at pump outlet & Ensure proper fluid flow \\
Flow rate measurements & Detection of any FLiNaK leakage into rotor or stator inner assemblies \\
Temperature measurements inside the & Test adequate pre-heating and cooling of stator \\
motor & \\
\hline
\end{tabular}

Table 16. Continuous Built-in Test

\begin{tabular}{ll}
\hline \multicolumn{1}{c}{ Parameters to monitor } & \multicolumn{1}{c}{ Purpose } \\
\hline Stator winding current & Ensure proper functioning of pumping system \\
Voltage & $\begin{array}{l}\text { Detect open circuits or electrical faults } \\
\text { Predict potential failure }\end{array}$ \\
Temperature & $\begin{array}{l}\text { Provide warning in the case of unexpected measurements } \\
\text { X-Y-Z position }\end{array}$ \\
Angular velocity & unexpected failure \\
Bearing forces & \\
Rotor torque & \\
Pump pressure & \\
Fluid flow rate in gap region and inside shaft & \\
\hline
\end{tabular}

\subsubsection{Prognostics and Anticipated Time to Failure}

The parameters of the pump operation should be recorded as described in Section 5.2. The historical record of these parameters (such as temperature, loads, duration of operation, record of faults, etc.) should be used to estimate the anticipated time to failure. It is desired that the motor operate within specification for 7 years continuously without the need of maintenance. The US Nuclear Regulatory Commission (NRC) requires cooling pump reliability to produce core melt frequency (CMF) and large release frequency (LRF) reliability and safety to be 1E-4/year and 1E-7/year, respectively.

\section{SYSTEM CONCEPTUAL DESIGN}

This section describes the basic motor design with its embedded features. Much of the design features in this section refer back to explanations developed in the previous report sections. The conceptual design is not a recipe for constructing a motor system but a step in that direction. Considerable further work is needed to expound the details to a level suitable for a construction project. 


\subsection{FUNCTIONAL DESCRIPTION}

The conceptual mechanical design is a horizontal shaft, single-stage, centrifugal, high-temperature, liquid pump that is compatible with liquid fluoride salts. Figure 73 depicts the functional concept of an impeller pump on the same shaft as an electric motor. The motor will feature both a canned rotor and active magnetic suspension bearings. In a canned rotor pump, the rotor of the pump motor is located entirely within the liquid, and thin-walled containers separate both the rotor and stator from the fluid. All of the stator windings are isolated from the fluid by the stator can. In a magnetic bearing pump, the rotor is levitated using active magnetic bearings. This design does not have any rotating seals and does not have any contacting moving elements.

The pump motor will employ a switched reluctance-type drive (SRM). Reluctance motors have the simplest rotors of all electric machines. The control of switched reluctance motors is more intimately coupled to the machine design than in other motors. Reluctance motors do not require permanent magnets or rotor windings, both of which are problematic for high-temperature environments.

As the pump shaft needs to be positioned horizontally, vertically, and axially, the pump will have x-axis, $\mathrm{y}$-axis, and z-axis magnetic bearings. All the stator windings (main rotational drive, radial bearings, and axial bearings) will be driven by power electronics in a separate enclosure maintained at normal electronics environment.

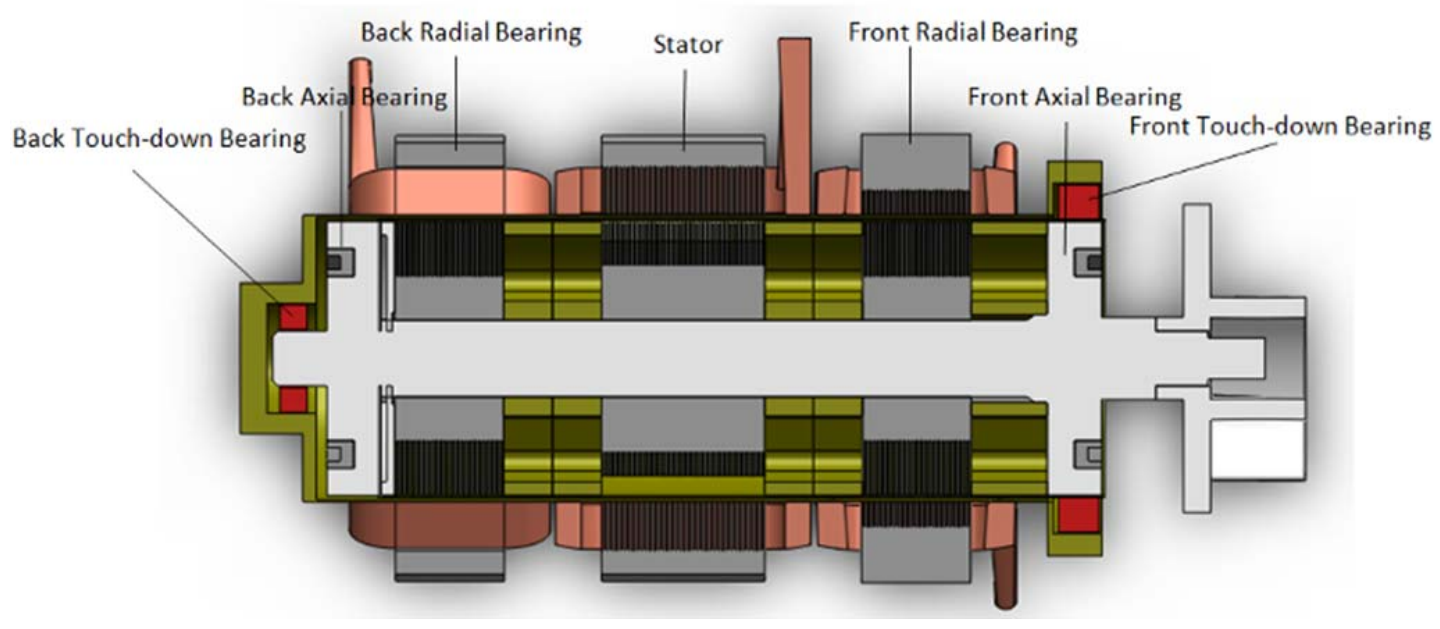

Fig. 73. Canned rotor pump concept.

\subsection{MAGNETIC BEARING DESIGN}

Magnetic suspension is an active system requiring continuous feedback control, as discussed previously. This section develops the theory for the forces and current drive needed for magnetic suspension. Figure 74 illustrates the path of the magnetic flux in a multi-pole bearing design, especially the series nature of the reluctances stacked across the gap. The nomenclature in the figure is given in Equation (73). 

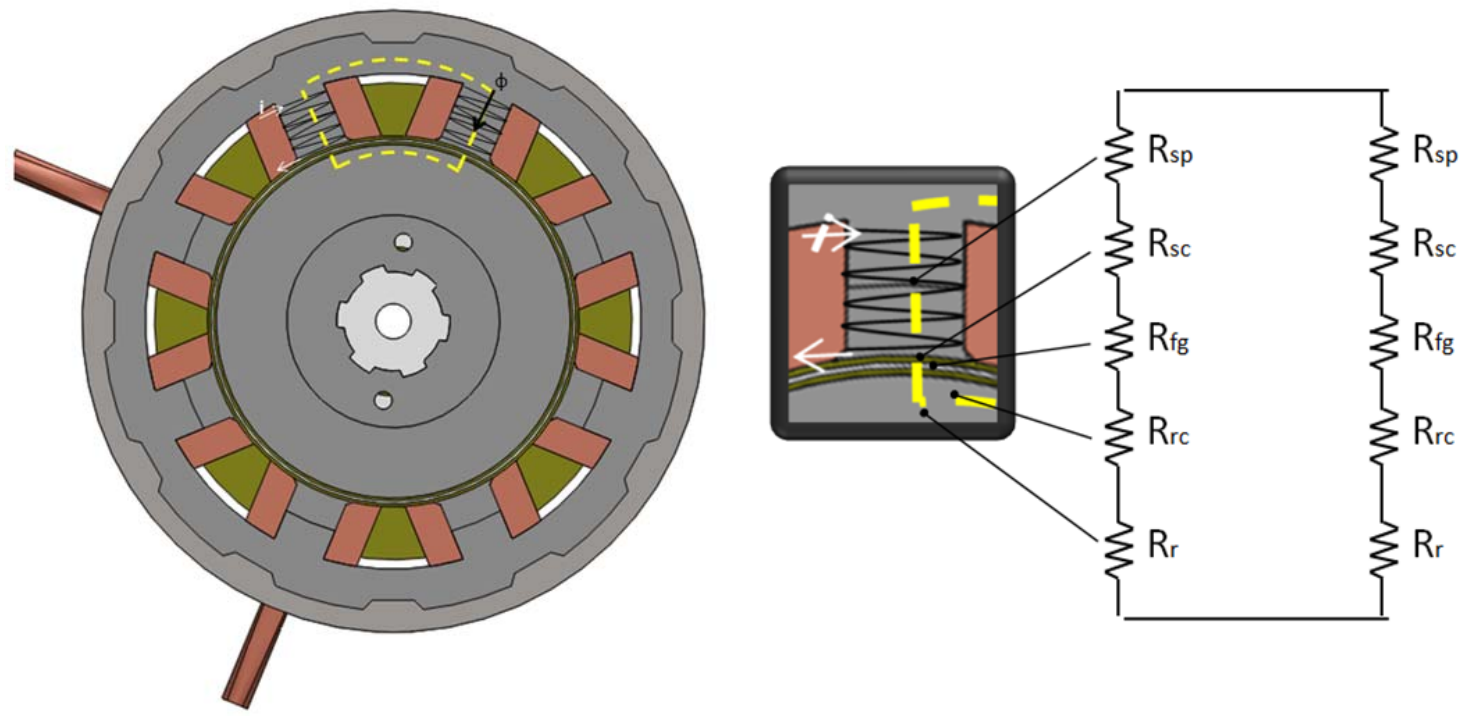

Fig. 74. Magnetic path illustration.

The magnetic reluctance of each component is given by Equation (72).

$$
R=\frac{t}{\mu A},
$$

where $t$ refers to the thickness of each component, A is the high flux cross-sectional area, and $\mu$ is the magnetic permeability of the material.

Assuming the high flux area is the stator pole cross-sectional area and is the same for the flux passing through the stator can, fluid gap, and rotor can, the total reluctance in each side of the magnetic flux path can be expressed as Equation (73).

$$
\begin{aligned}
\sum R=R_{\text {stator pole }}+R_{\text {rotor can }}+R_{\text {fluid gap }}+R_{\text {strator can }}+R_{\text {rotor }} \\
=\frac{t_{\text {strator pole }}}{\mu_{\text {stator }} A}+\frac{t_{\text {strator can }}}{\mu_{\text {stator can }} A}+\frac{t_{\text {fluid gap }}}{\mu_{\text {FLiNaK }} A}+\frac{t_{\text {rotor can }}}{\mu_{\text {rotor can }} A}+\frac{t_{\text {bearing rotor section }}}{\mu_{\text {bearing rotor }} A} .
\end{aligned}
$$

Note that all thickness values are fixed with the exception of the fluid gap (red). Since the reluctances are in parallel, the total reluctance for the magnetic loop is

$$
R_{\text {total }}=\frac{\sum R^{2}}{2\left(\sum R\right)},
$$

And the contribution from all three components (stator can, rotor can and fluid gap) is taken into account. The magnetic flux is given by 


$$
\Phi=\frac{N i}{R_{\text {total }}} .
$$

The flux linkage is given by

$$
\lambda=N \Phi \frac{N^{2} i}{R_{\text {total }}}
$$

The inductance of the circuit can be found by Equation (77),

$$
L=\frac{\lambda}{i}
$$

and the inductance per unit area can be found by Equation (78),

$$
\frac{L}{A}=\frac{N^{2}}{R_{\text {total }} A} .
$$

Solving for the inductance per unit area, assuming 100 turns on the coil, and a gap range from $0.2-1 \mathrm{~mm}$, the reluctance and inductance per area curves are generated and shown in Figs. 75 and 76.

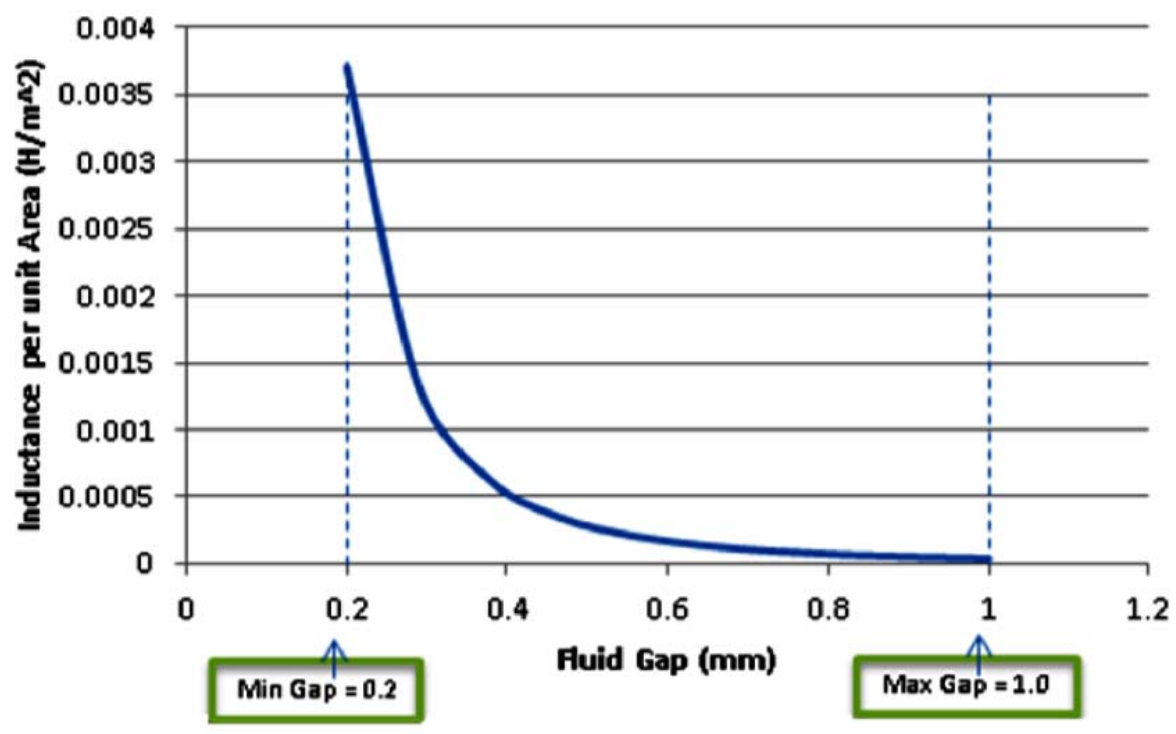

Fig. 75. Relationship of inductance to gap. 


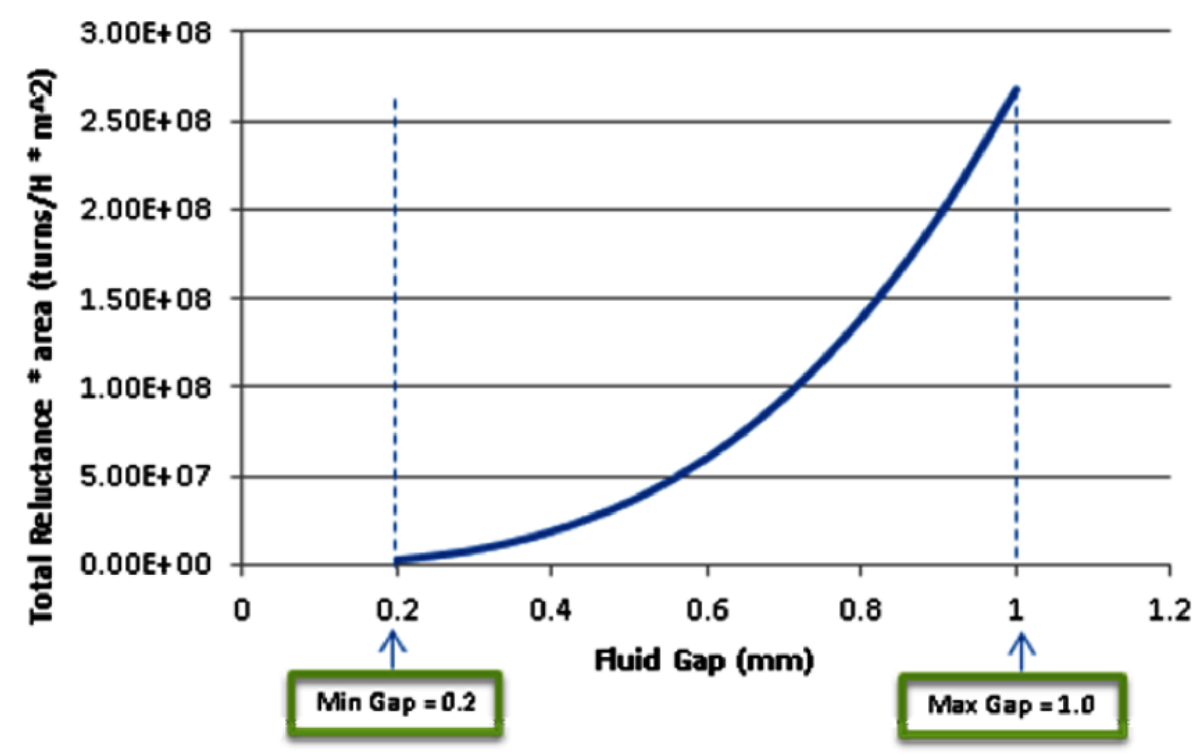

Fig. 76. Relationship of reluctance to gap.

To estimate the number of turns required for the active magnetic bearings, the magnetic bearing force is derived. The stored magnetic energy is given by Equation (79):

$$
w_{m}=\int_{0}^{\lambda_{o}} i d \lambda
$$

Magnetic co-energy is obtained by

$$
w_{m}^{\prime}=\int_{0}^{i_{o}} i d \lambda
$$

Since the work done by the magnetic force is $F \times \delta x$, the force is given by

$$
F=\frac{\partial W_{m}^{\prime}}{\partial x}=\frac{\partial L}{\partial x}\left(\frac{i^{2}}{2}\right) .
$$

Then by ignoring the contributions to inductance by the rotor, stator, and cans, the inductance is given by Equations (82) and (83).

$$
\begin{aligned}
& L=\frac{N^{2} \mu_{0} A}{2(g-x)} \\
& \frac{\partial L}{\partial x}=\frac{N^{2} \mu_{0} A}{2 g^{2}} .
\end{aligned}
$$

Hence, the electromagnetic force can be expressed as Equation (84). 


$$
F=\frac{N^{2} i^{2} \mu_{0} A}{4 g^{2}} .
$$

Given a maximum required force that bearings must counteract (gravitational load, moment from impeller rotation, and maximum disturbance forces), and preliminary geometrical values, the number of turns may be estimated relative to the input current.

The active magnetic bearings must be able to counteract the following forces.

- rotor weight

- disturbance force

- tangential force from rotor torque

For an estimated force requirement of $291 \mathrm{~N}$ and a fluid gap of $1 \mathrm{~mm}$, the plot for required turns-vs.current is given in Fig. 77 for a range of 10-100 amps.

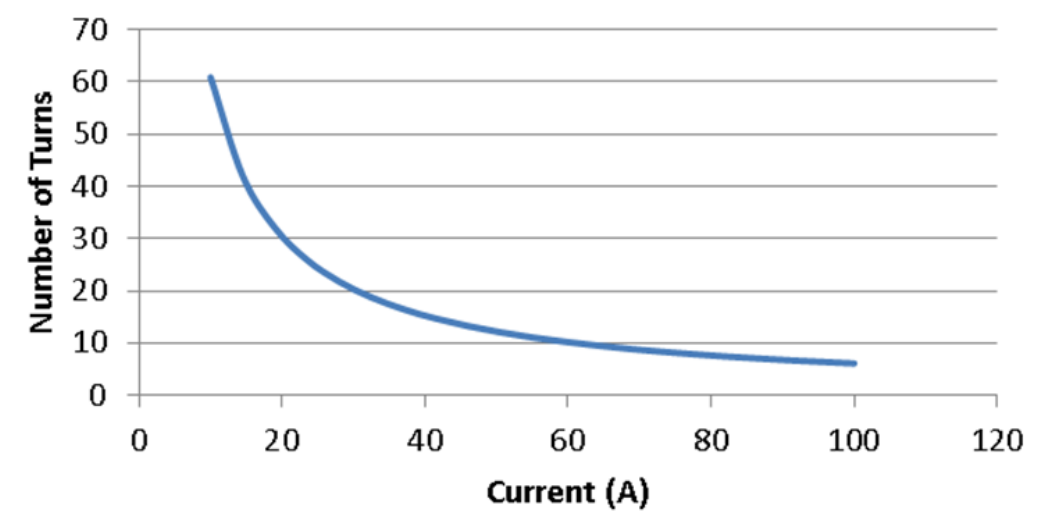

Fig. 77. Required number of turns vs. input current for constant force and $1 \mathrm{~mm}$ gap.

Similarly, a range of number of turns may be estimated for varying fluid gap, for different input currents (Fig. 78).

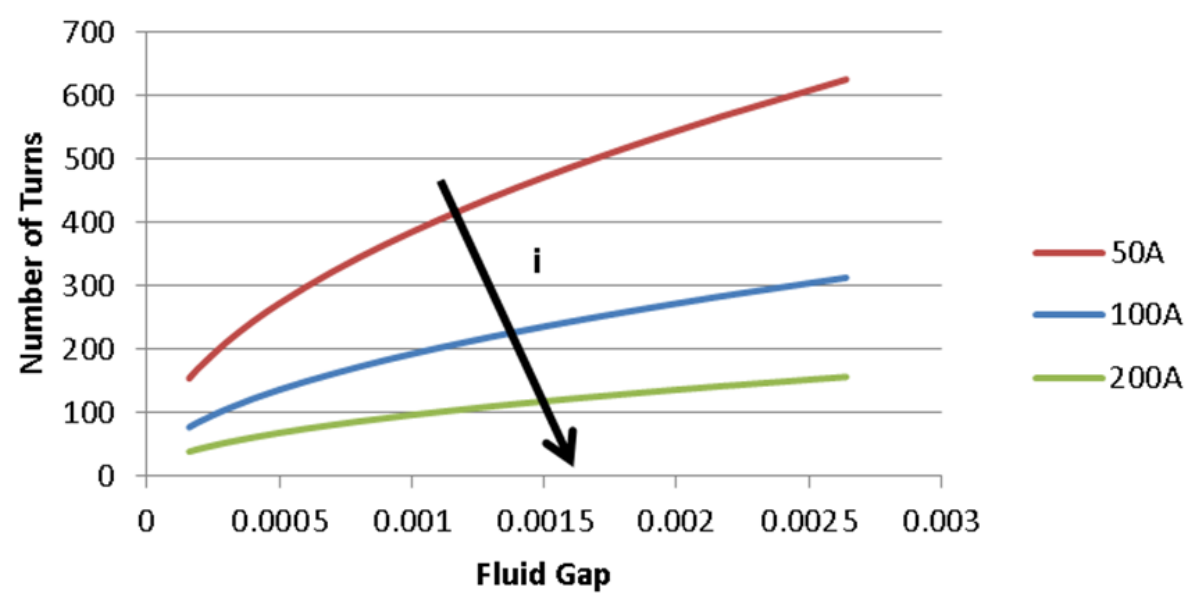

Fig. 78. Required number of turns for constant input current over a fluid gap range. 
The number of turns can also be estimated for varying required forces for a given fluid gap (Fig. 79).

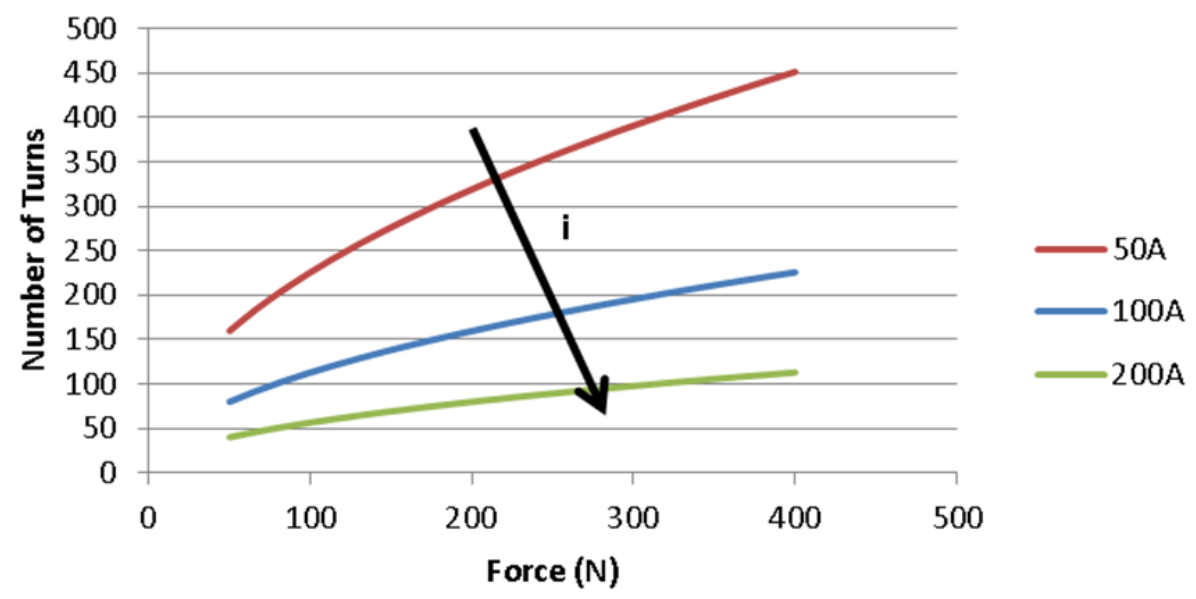

Fig. 79. Required number of turns for a range of electromagnetic forces at three different input currents, for a gap of $1 \mathrm{~mm}$.

For a given number of coils, and a $1 \mathrm{~mm}$ fluid gap, the current required to provide the required electromagnetic force may be estimated (Fig. 80).

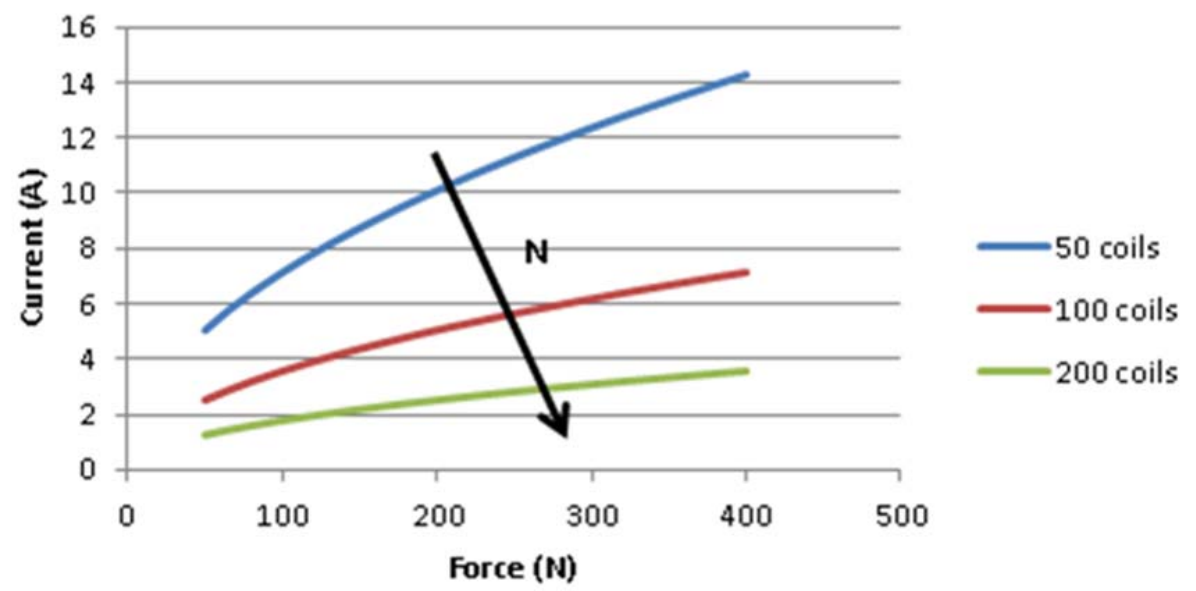

Fig. 80. Required current to provide electromagnetic force for a given number of coils $(\mathrm{N})$ and to maintain a $1 \mathrm{~mm}$ gap.

\subsection{CONTROL SYSTEMS}

The extreme environment provided by the high-temperature canned rotor test bed demands the application of advanced control system design techniques. During the first phase of controller design, the system model will be derived from a combination of first principles and simulations. The second phase of controller design will include a test bed for model identification and validation along with control algorithm testing. There are a number of unique issues involved in the control system design driven by this particular application. Many of the assumptions in traditional control theory applied to active magnetic bearings no longer hold. The extreme temperature variations in the operating range means that material properties are no longer static. Additionally, the high temperature means that many materials will age, causing permanent changes to the electromagnetic and mechanical dynamics of the system that must 
be accounted for. The addition of rotor and stator cans in the magnet and motor flux paths causes an input time delay and bandwidth limitation due to the generation and decay of eddy current. The large air gap required due to physical constraints on the system causes non-linearities from saturation effects. The bearing and motor dynamics are coupled, and this cross correlation needs to be addressed in the control system design. The novel sensor design also introduces uncertainties and noise into the control system. These uncertainties cause an effective decrease in the sensor bandwidth, limiting the available bandwidth for the controller, which in turn limits the minimum magnetic gap while the force requirements on the bearings and motor limit the maximum air gap. In certain configurations, this means that the design is unrealizable. Under these conditions, a simple PID controller on each bearing axis will be unable to stabilize the system. Additionally, these simple control system design techniques do not provide the ability to operate with degraded performance or graceful shutdown under component failure.

The rotor dynamics will be modeled as a flexible rotor. The amplifier, actuator (coil), and sensor dynamic models will be developed and included into the system model. The novel sensor techniques used will require significant signal conditioning and analysis that must be incorporated into the system dynamics. The time delay caused by eddy currents in the stator and rotor can is included in the actuator dynamics. Given the derived system model, robust control techniques will be used to develop and refine a controller to stabilize the system and maintain the performance requirements in the presence of the sensor noise, sensor uncertainty, time delays, and model uncertainty. Specifically $\mathrm{H}_{\infty}$ and $\mu$-synthesis control design techniques will be applied. Next, a supervisory controller will be developed around the nominal robust controller to handle start-up, shutdown, safety, nominal operations, and operations under degraded conditions. The closed-loop system will be initially verified through simulation and then implemented on a test bed.

This system will also incorporate a supervisory control system in addition to the multi-input/multi-output (MIMO) bearing and motor control as shown in Fig. 81. A representative motor control loop is shown in Fig. 82 . 


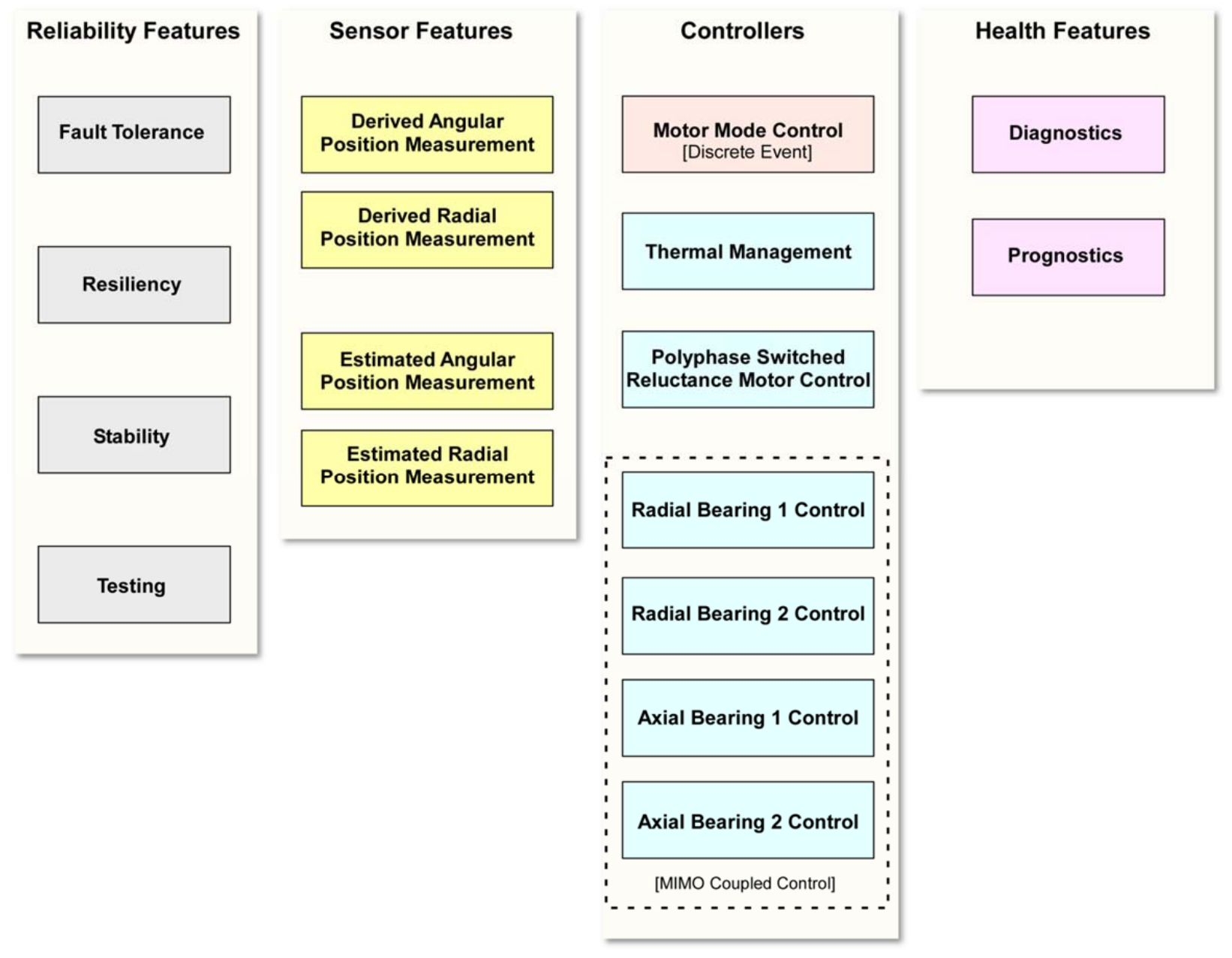

Fig. 81. Control and signal-processing components of embedded motor system summarized at a high level.

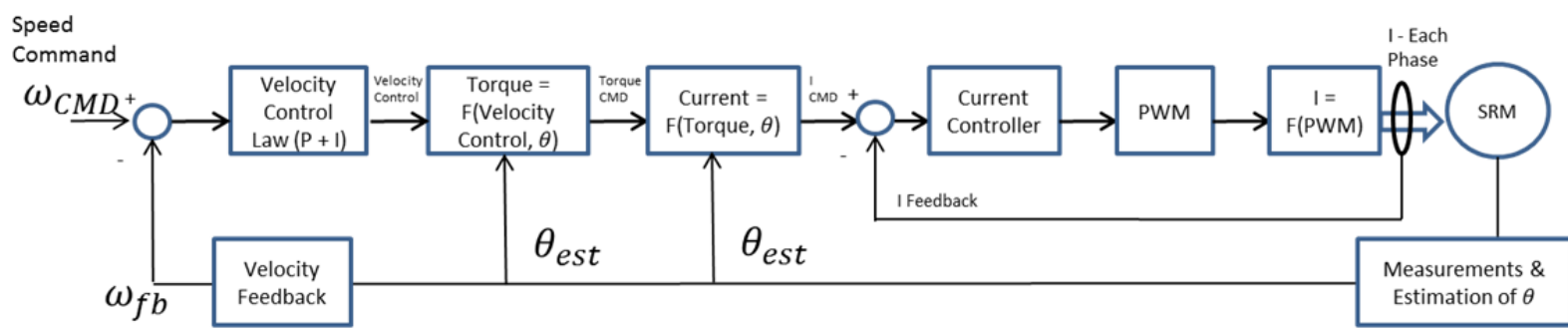

Fig. 82. Switched reluctance motor speed control block diagram showing loop gain stages.

\subsubsection{Bearing Control System}

The magnetic bearings control system must provide the proper transient response to maintain the desired shaft position as the shaft is disturbed by the pump load, shaft unbalance, and motor torque ripple.

The magnetic bearing control system functional block diagram for the radial bearing case is shown in Fig. 83. This figure depicts a PID control scheme; however, the control system necessary for the high- 
temperature canned rotor pump will be more sophisticated. The radial and axial bearings will utilize the same control scheme and architecture.

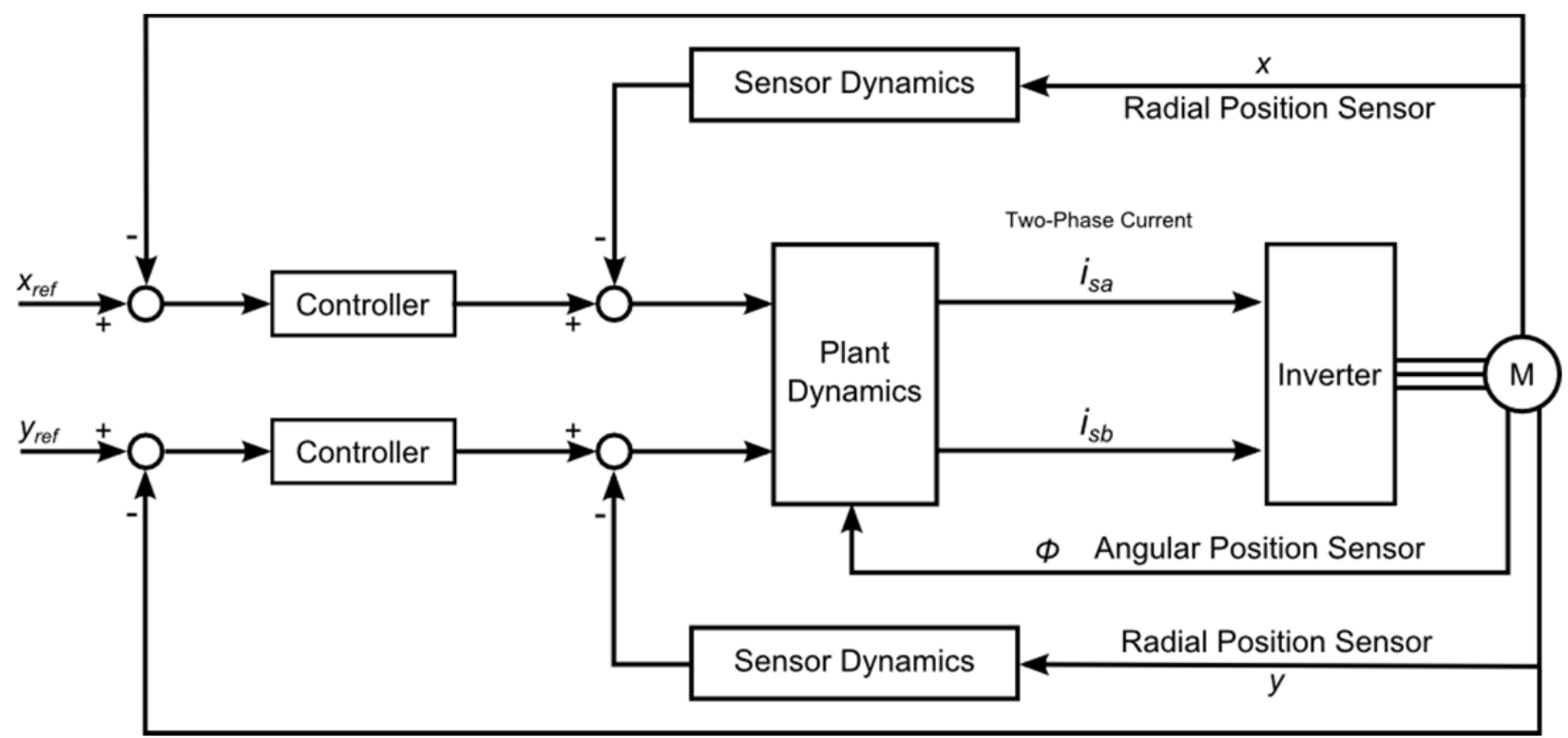

Fig. 83. Bearing control system block diagram (radial case).

The step response should have a rise time not exceeding 2 milliseconds and a settling time not exceeding 4 milliseconds (Fig. 84). The closed loop frequency response will have a bandwidth of $1 \mathrm{kHz}$ (Fig. 85).

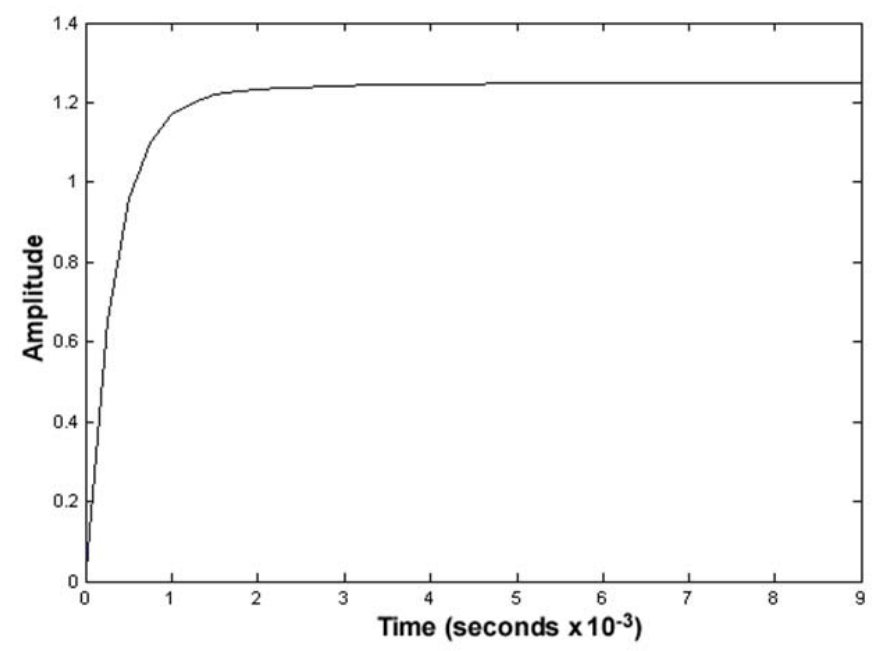

Fig. 84. Bearing system step response. 

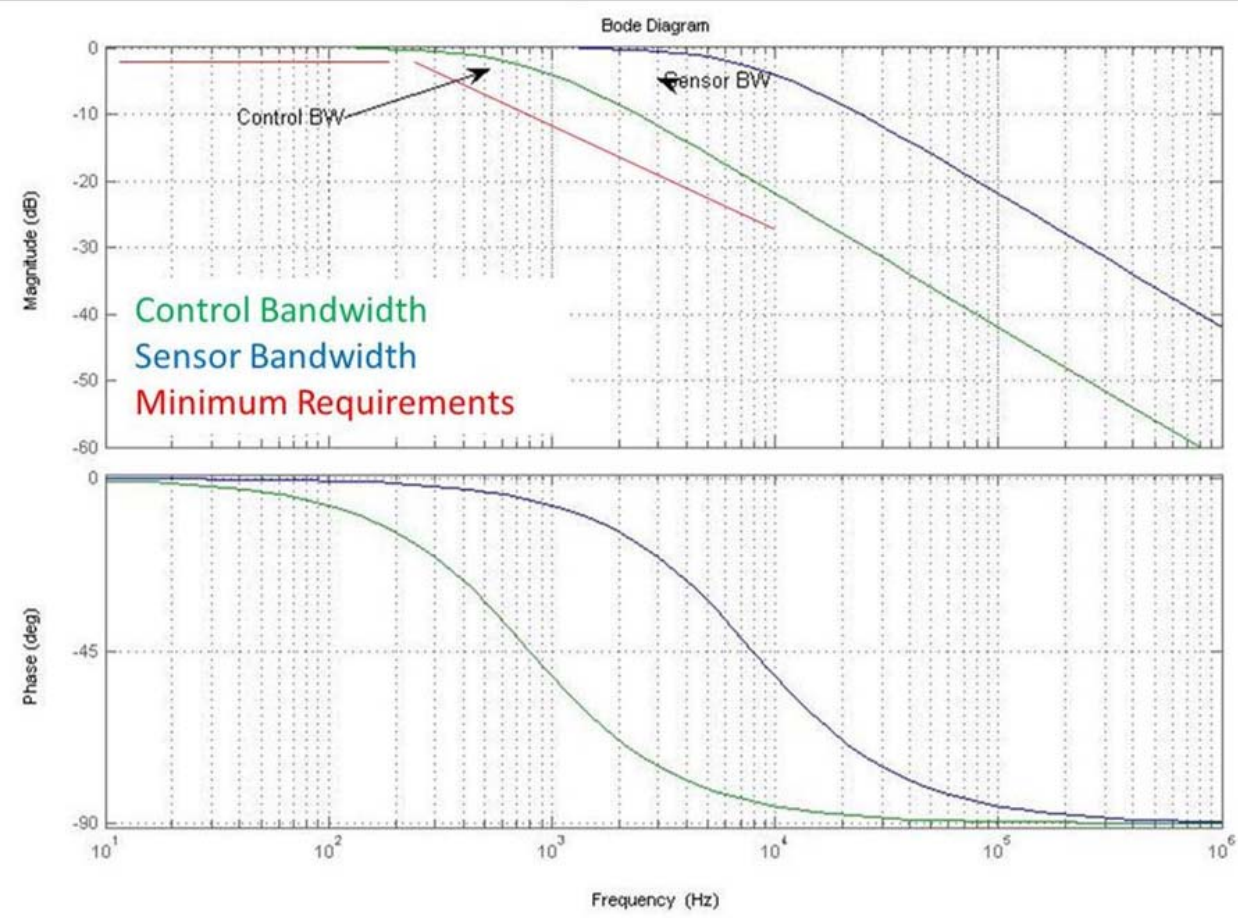

Fig. 85. Closed loop gain and phase estimations for bearing control.

\subsubsection{Control Algorithms}

A number of factors should be considered when choosing control algorithm design methodology: (1) the demonstration platform is temperature dependent and time varying; (2) the requirement that the sensors not penetrate the stator can will add noise and significant dynamics to the measurements; and (3) time delays are introduced due to eddy current formation and dissipation in the stator and rotor cans. These factors limit the workable control algorithms. For example to meet the performance requirements, it will not be possible to decouple the axes and use PID control. The axis coupling and the structural dynamics of the rotor bending modes need to be included into the system dynamics. Similarly, full state optimal feedback will most likely be insufficient, depending upon the noise and uncertainty in the system. Additionally, the physical coupling between the rotor dynamics and the sensors needs to be correlated to estimate the rotor states will sufficient accuracy to fulfill the performance requirements. The most likely candidates for the control algorithm design is $\mathrm{H}_{\infty}$ or $\mu$-synthesis robust control design techniques.

\subsection{SENSORS}

Typical magnetic bearing systems employ position-sensing techniques such as an air gauging, inductive Eddy current, capacitive, optical (photonic), Hall element, ultrasonic, or a laser triangulation measurement system. ${ }^{51,52}$ The bearing position sensors must provide the following characteristics.

1. Non-contact measurement

2. Bandwidth of the sensor must exceed the control loop and bearing

3. Precise mechanical alignment

4. Temperature stability

5. Isolated from bearing magnetic fields

6. Desired measurement accuracy and precision $^{53}$ 
Eddy current sensors require an electrically conductive target. These sensor types are widely used for magnetic bearing applications. ${ }^{53}$

The environment factors of high temperature $\left(700^{\circ} \mathrm{C}\right)$ limit the materials used for the motor, bearings, and structure. Permanent magnets are not suitable for the temperature range, which limits sensing methods such as Hall Effect. Capacitive bearing sensors can be constructed of materials to operate up to $1200^{\circ} \mathrm{C}^{52}$ but have significant phase shift. ${ }^{51}$ Inductive eddy current sensors are available commercially up to $600^{\circ} \mathrm{C}^{52}$

The pump barrier concept, a separate rotor and stator can with a layer of molten salt in between, does not permit any optical or electrical contact with the bearing rotor for measurement purposes. Techniques such as ultrasonic, capacitance, or inductive eddy current must be able to measure the bearing rotor position by sensing through the layers of the rotor can, salt, and stator can.

Typical motor systems utilize sensors such as encoders, resolvers, or tachometers that have a mechanical coupling with the motor shaft. The pump concept of rotor and start can layers with a layer of molten salt in between does not permit the coupling of any sensing devices with the rotating parts.

Magnetic bearing systems that do not use physical sensors are described as self-sensing or sensorless. Sensorless techniques are described in References 53, 54, and 55.

1. PWM current carrier frequency

2. Differential transformer with a push-pull electromagnet

3. Induction sensing of magnetic coil to determine bearing gap

4. High-frequency signal injection on magnetic coil

The control of an SRM is dependent on knowledge of the relative and absolute rotor position. ${ }^{56}$ Motivations such as financial cost, harsh environments, and other factors have led to research in sensorless control of SRM machines. The fundamental principle of SRM sensor-less methods is that rotor position information can be obtained from the stator circuit measurements (voltage and current at the motor terminals) or their derived parameters (phase, inductance, or others) ${ }^{57}$

SRM rotor sensorless measurements can be described in three broad classes.

1. Observer-based schemes that estimate rotor position

2. Inductance-based measurements that use the current rise time and fall time to obtain discretely the rotor position

3. Inductance-based estimation of rotor position constant current or flux linkage applied to sensor signals and sensing phases ${ }^{57}$

SRM sensorless methods can also be classified as follows.

1. Energized phase - Chopping waveform, regenerative current, flux linkage, state observers, irregularities in stator/rotor poles, and current waveform methods

2. Unenergized phase - Active probing, modulated signal injection, regenerative current, and mutually induced systems

3. Open loop methods - Dwell angle compensation and commutation angle compensation ${ }^{57}$

Inductive-based measurement systems have a three-dimensional relationship between inductance, current, and rotor position. Knowing current and inductance can facilitate resolving the rotor position. 


\subsubsection{Bearing Sensors and Instrumentation}

To control the position of the rotor can with respect to the stator can through the magnetic bearings, the relative position of the rotor can at that location must be recorded. For this purpose, two sensors in the $\mathrm{x}$-direction, and two sensors in the y-direction will be implemented for the radial bearings. Measurements for axial position will also be recorded, as an axial bearing will control position in the z-direction. Additionally, the angular position of the rotor with respect to the stator will be recorded as well, since speed control and proper drive of the switch-reluctance motor will depend on angular position. Table 17 is a summary of all measurements and their respective required specifications.

Table 17. Uncertainty values for bearing control

\begin{tabular}{|c|c|c|c|c|c|c|}
\hline $\begin{array}{c}\text { Sensor } \\
\text { Location }\end{array}$ & Measurement & Purpose & $\begin{array}{c}\text { Required } \\
\text { precision }\end{array}$ & $\begin{array}{l}\text { Required } \\
\text { accuracy }\end{array}$ & $\begin{array}{c}\text { Required } \\
\text { sensor } \\
\text { sensitivity }\end{array}$ & Range \\
\hline \multirow{4}{*}{$\begin{array}{c}\text { Back } \\
\text { Radial } \\
\text { Bearing }\end{array}$} & $\begin{array}{l}x 1-\text { Relative shaft position } \\
\text { in } x\end{array}$ & & $0.01 \mathrm{~mm}$ & $0.25 \mathrm{~mm}$ & $0.1 \mathrm{~V} / \mathrm{mm}$ & $6 \mathrm{~mm}$ \\
\hline & $\begin{array}{l}\text { x2- Relative shaft position } \\
\text { in } \mathrm{x}\end{array}$ & & $0.01 \mathrm{~mm}$ & $0.25 \mathrm{~mm}$ & $0.1 \mathrm{~V} / \mathrm{mm}$ & $6 \mathrm{~mm}$ \\
\hline & $\begin{array}{l}\text { y1- Relative shaft position } \\
\text { in y }\end{array}$ & & $0.01 \mathrm{~mm}$ & $0.25 \mathrm{~mm}$ & $0.1 \mathrm{~V} / \mathrm{mm}$ & $6 \mathrm{~mm}$ \\
\hline & $\begin{array}{l}\text { y2- Relative shaft position } \\
\text { in y }\end{array}$ & & $0.01 \mathrm{~mm}$ & $0.25 \mathrm{~mm}$ & $0.1 \mathrm{~V} / \mathrm{mm}$ & $6 \mathrm{~mm}$ \\
\hline \multirow{4}{*}{$\begin{array}{l}\text { Front } \\
\text { Radial } \\
\text { Bearing }\end{array}$} & $\begin{array}{l}\mathrm{x} 1 \text { - Relative shaft position } \\
\text { in } \mathrm{x}\end{array}$ & & $0.01 \mathrm{~mm}$ & $0.25 \mathrm{~mm}$ & $0.1 \mathrm{~V} / \mathrm{mm}$ & $6 \mathrm{~mm}$ \\
\hline & $\begin{array}{l}\text { x2- Relative shaft position } \\
\text { in } x\end{array}$ & $\begin{array}{l}\text { Control of } x-y-z \\
\text { position of shaft }\end{array}$ & $0.01 \mathrm{~mm}$ & $0.25 \mathrm{~mm}$ & $0.1 \mathrm{~V} / \mathrm{mm}$ & $6 \mathrm{~mm}$ \\
\hline & $\begin{array}{l}\text { y1- Relative shaft position } \\
\text { in y }\end{array}$ & bearings. & $0.01 \mathrm{~mm}$ & $0.25 \mathrm{~mm}$ & $0.1 \mathrm{~V} / \mathrm{mm}$ & $6 \mathrm{~mm}$ \\
\hline & $\begin{array}{l}\text { y2- Relative shaft position } \\
\text { in y }\end{array}$ & & $0.01 \mathrm{~mm}$ & $0.25 \mathrm{~mm}$ & $0.1 \mathrm{~V} / \mathrm{mm}$ & $6 \mathrm{~mm}$ \\
\hline \multirow{4}{*}{$\begin{array}{c}\text { Axial } \\
\text { Bearing }\end{array}$} & $\begin{array}{l}\text { z1- Relative shaft position in } \\
\mathrm{z}\end{array}$ & & $0.01 \mathrm{~mm}$ & $0.25 \mathrm{~mm}$ & $0.1 \mathrm{~V} / \mathrm{mm}$ & TBD \\
\hline & $\begin{array}{l}\text { z2- Relative shaft position in } \\
z\end{array}$ & & $0.01 \mathrm{~mm}$ & $0.25 \mathrm{~mm}$ & $0.1 \mathrm{~V} / \mathrm{mm}$ & TBD \\
\hline & $\begin{array}{l}\text { z3- Relative shaft position in } \\
\text { z }\end{array}$ & & $0.01 \mathrm{~mm}$ & $0.25 \mathrm{~mm}$ & $0.1 \mathrm{~V} / \mathrm{mm}$ & TBD \\
\hline & $\begin{array}{l}\text { z4- Relative shaft position in } \\
\text { z }\end{array}$ & & $0.01 \mathrm{~mm}$ & $0.25 \mathrm{~mm}$ & $0.1 \mathrm{~V} / \mathrm{mm}$ & TBD \\
\hline Stator & $\begin{array}{l}\theta \text { - Relative angular position } \\
\text { of rotor poles with respect to } \\
\text { stator poles. }\end{array}$ & $\begin{array}{l}\text { Control of } \\
\text { rotational speed } \\
\text { through stator. }\end{array}$ & & $0.3^{\circ}$ & & $0-360^{\circ}$ \\
\hline
\end{tabular}

With a desired fluid gap of $1 \mathrm{~mm}$, the maximum can displacement is $2 \mathrm{~mm}$, and hence $\mathrm{x}$ and $\mathrm{y}$ displacement sensors must have a minimum range of $2 \mathrm{~mm}$. Nevertheless, sensors will be placed outside of the stator can and measurements will be taken through it, so can thicknesses, tolerances, and thermal 
expansion should be taken into consideration when determining the required measurement range. Taking those factors into account, an approximate minimum sensing range of $6 \mathrm{~mm}$ has been estimated (Fig. 63).

Given that the maximum allowable displacement for the rotor can is $0.5 \mathrm{~mm}$, the measurement precision of this gap should be $0.01 \mathrm{~mm}$. Assuming a signal-to-noise ratio of 100 , an acceptable noise of $1 \mu \mathrm{V}$, and a desired precision of $0.01 \mathrm{~mm}$, the required sensor sensitivity for $\mathrm{x}-\mathrm{y}$ displacement sensors is $0.1 \mathrm{~V} / \mathrm{mm}$.

The magnetic bearing sensor will be sampled at a minimum of $5 \mathrm{kHz}$ and a maximum of $10 \mathrm{kHz}$. See Section 6.3.1.

There is an upper bound on the operating frequency of the different signals that will be used in the system because of generating eddy currents on the stator can surface, which prevents penetration of the magnetic field. This upper bound varies by material and thickness as shown in Fig. 86. Balancing the possible operating frequencies for the various magnetic subsystems allows creating a bandwidth budget, as summarized in Fig. 87.

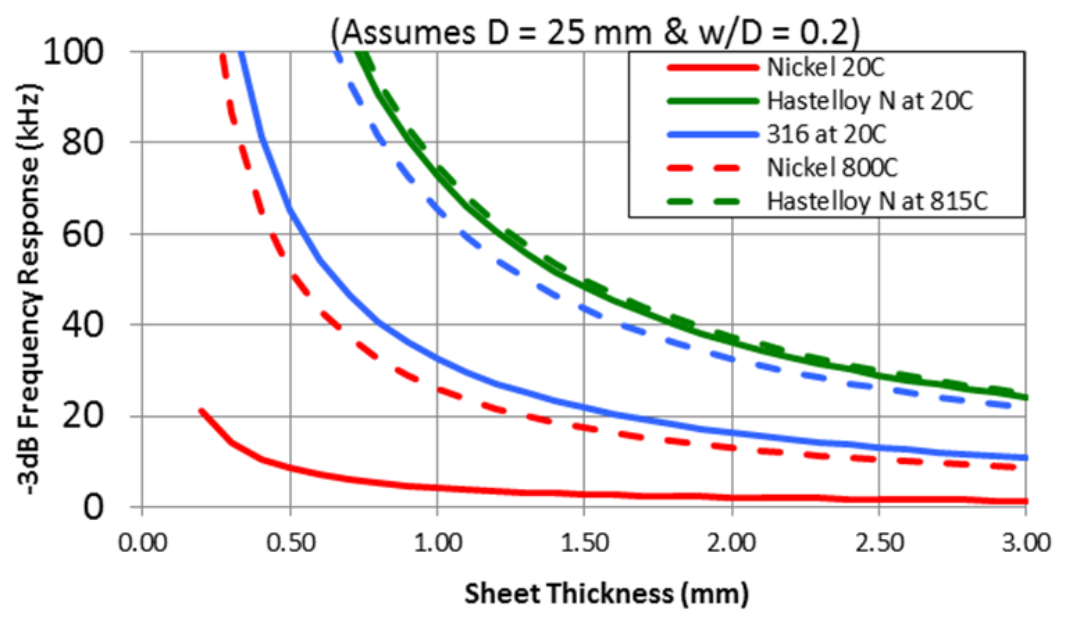

Fig. 86. Frequency response as a function of sheet thickness.

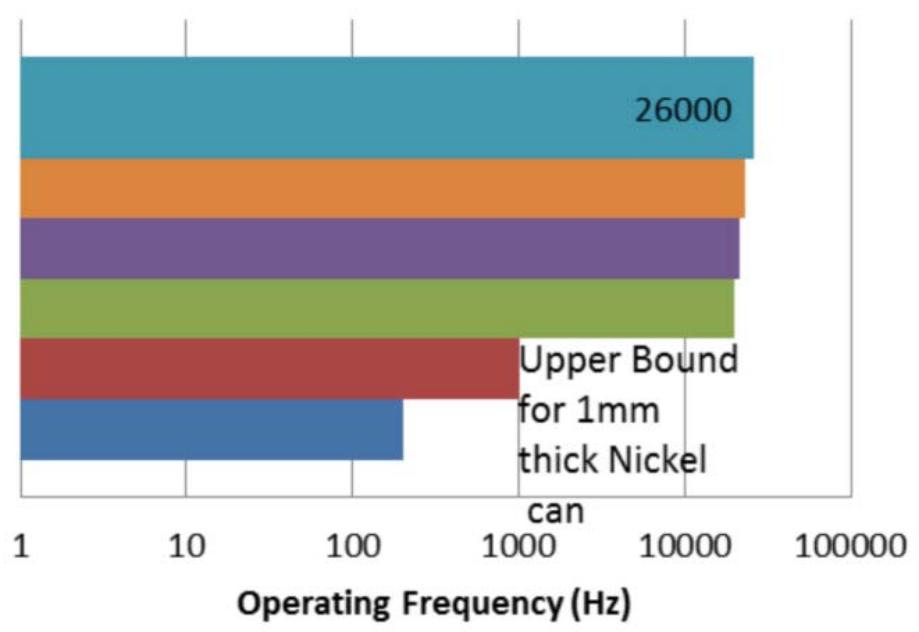

Bearing Sensor y1

Bearing Sensor $\mathrm{y} 1$

Bearing Sensor $x 2$

Bearing Sensor $x 1$

Bearing PWM

Bearing Drive

Fig. 87. Bearing bandwidth budget.

Assuming a 1-mm-thick nickel can at $800^{\circ} \mathrm{C}$, the maximum frequency is approximately $25.9 \mathrm{kHz}$. Several components of the system will require current injection for either driving a system, or for measurement. 
At close proximity, it is important to avoid interference, and hence, there must be separation between frequencies. The following plot displays the potential frequencies present within one of the radial bearings, assuming a PWM drive frequency of $1 \mathrm{kHz}$, and sensors beginning at $20 \mathrm{kHz}$, assuming inductive sensors are used. In this plot, the $\mathrm{x}$ and $\mathrm{y}$ sensor frequencies are equally separated and within their minimum and maximum values. It must be noted, however, that this difference in frequency may not be sufficient to avoid interference. A list of motor processes and the estimated frequency ranges is given in Table 18.

Table 18. List of processes requiring input currents and associated frequencies

\begin{tabular}{|c|c|c|}
\hline Current injection location & Process & Potential frequency range \\
\hline \multirow{8}{*}{ Front radial bearing } & $\mathrm{x} 1$ Measurement & $20-100 \mathrm{kHz}$ \\
\hline & x2 Measurement & $20-100 \mathrm{kHz}$ \\
\hline & y1 Measurement & $20-100 \mathrm{kHz}$ \\
\hline & y2 Measurement & $20-100 \mathrm{kHz}$ \\
\hline & x1 Drive & $1 \mathrm{kHz}$ \\
\hline & $\mathrm{x} 2$ Drive & $1 \mathrm{kHz}$ \\
\hline & y1 Drive & $1 \mathrm{kHz}$ \\
\hline & y2 Drive & $1 \mathrm{kHz}$ \\
\hline \multirow{4}{*}{ Stator } & Theta Measurement & $20-100 \mathrm{kHz}$ \\
\hline & Pole pair Drive 1 & $10 \mathrm{kHz}$ \\
\hline & Pole pair Drive 2 & $10 \mathrm{kHz}$ \\
\hline & Pole pair Drive 3 & $10 \mathrm{kHz}$ \\
\hline \multirow{8}{*}{ Back radial bearing } & x1 Measurement & $20-100 \mathrm{kHz}$ \\
\hline & x2 Measurement & $20-100 \mathrm{kHz}$ \\
\hline & y1 Measurement & $20-100 \mathrm{kHz}$ \\
\hline & y2 Measurement & $20-100 \mathrm{kHz}$ \\
\hline & x1 Drive & $1 \mathrm{kHz}$ \\
\hline & x2 Drive & $1 \mathrm{kHz}$ \\
\hline & y1 Drive & $1 \mathrm{kHz}$ \\
\hline & y2 Drive & $1 \mathrm{kHz}$ \\
\hline \multirow{6}{*}{ Axial bearing } & z1 Measurement & $20-100 \mathrm{kHz}$ \\
\hline & z2 Measurement & $20-100 \mathrm{kHz}$ \\
\hline & z3 Measurement & $20-100 \mathrm{kHz}$ \\
\hline & z4 Measurement & $20-100 \mathrm{kHz}$ \\
\hline & z1-z2 Drive & $1 \mathrm{kHz}$ \\
\hline & z3-z4 Drive & $1 \mathrm{kHz}$ \\
\hline
\end{tabular}




\subsection{MOTOR PHYSICAL COMPONENTS}

\subsubsection{Rotor}

The use of magnetic bearings will allow the rotor to be built as a monolithic unit and inserted into the stator housing.

\section{Shaft}

The exposure of the shaft to the corrosive effects of the salt and the strength requirements mean that the most suitable material is Inconel ${ }^{\circledR} 600$. The shaft will be hollow for the return path of the coolant salt flow between the stator and rotor cans. The motor and bearing laminations will be coupled to the shaft. Using a keyway for torque transfer would exceed the rotor balance tolerance, so an axially symmetric system must be used. The most likely processes are a spline system or high-temperature brazing. An Inconel ${ }^{\circledR} 600$ shoulder the same diameter as the motor and bearing laminations will be mounted to the shaft at either end of the laminations to provide structural support for the laminations, the rotor can, and support for the $\mathrm{Fe}-\mathrm{Co}$ axial magnetic bearings. The thermal of expansion over the temperature range $\left(0-700^{\circ} \mathrm{C}\right)$ is not trivial and needs to be carefully accounted for in the design of the shaft and rotor. The reduction in strength of many of the materials in the rotor at $700^{\circ} \mathrm{C}$ coupled with the mismatches in their coefficients of thermal expansion can easily lead to rotor warping or failure if not properly accounted for.

\section{Magnetic Bearings and Motor}

The magnetic bearing and motor laminations will consist of a 0.014 in. thick Fe-Co alloy. The laminations will be laser cut and welded. After welding, they will be annealed to reduce stresses and homogenize the magnetic properties. The laminations will be separated on the shaft with spacers. Again, the thermal expansion in the axial and radial directions needs to be accounted for in the design of the part sizes and tolerances.

\section{Protective Barrier (inner)}

The rotor can will be made of Hastelloy ${ }^{\circledR} \mathrm{N}$ for its corrosion resistance properties. The protective can will be made in two parts that will then be welded together and to the axle. Any protrusions from the welding process will be turned smooth.

\subsubsection{Stator}

\section{Structural Components}

The stator will be built from the outer structural shell inward. The outer structural shell will provide the torque coupling for the stator laminations, attachment for the stator can, thermal management systems, and electrical feed through connections.

\section{Magnetic Bearings and Motor}

The magnetic bearings and motor laminations for the stator will be made in the same manner as the rotor laminations. 


\section{Windings and Electrical Connections}

The stator laminations will be wound externally then inserted into the outer structural shell. After all electrical connections have been built, the laminations, windings, and structural shell will be inserted onto a mandrel and then potted with a ceramic in an inert gas environment to seal the stator and provide structural support for the windings.

\section{Thermal Management}

Heat generation in the coils needs to be removed. Some heat will be removed through conductive transfer into the salt channel between the rotor can and the stator can. The remaining excess heat will be removed through either surface convection or active cooling, depending on the coil size and power input.

\section{Protective Barrier}

The stator protective can will be fabricated as one welded piece with a flange that will inserted into the potted lamination, coils, and structural shell. The stator can will support the touchdown bearings. The pump housing will be attached to the stator can flange and include a flexalitic gasket to provide the salt seal.

\subsection{PREHEATING SYSTEM}

The pump system must achieve a proper temperature to be compatible with the liquid-fluoride-salt medium. This requires a preheating capability to take the pump system from some initial condition to a temperature compatible with the salt medium. After preheating, continued heating of the pump system will occur due to the stator windings (electrical losses) and from the flow of salt internally in the pump during pumping operation.

In addition to preheating requirements, temperature limits for the various materials and stator windings must be considered. Heating up the internal pump structure to proper temperatures within a reasonable time without exceeding key material temperatures is a challenge. The heating apparatus may be applied in a temporary fashion to support preheating and then removed to enable the proper pump system cooling during pumping operation.

Several concepts are being examined. These concepts include the application of induction heating, which is shown in Fig. 88. Other concepts would be to apply a heating jacket or blanket device. Induction heating can be applied by separate solenoid coils inserted between the main drive stator and magnetic suspension bearing stators as shown. These coils would be wound on iron-cobalt pole pieces. The frequency would be chosen to heat the inner and outer cans selectively. Additionally, Inconel ${ }^{\circledR} 600$ pieces can be included in the rotor just beneath the induction coils to further heat the rotor assembly. An alternative approach is to use the normal stator windings as the heater by apply high-frequency signals to cause induction heating. This alternative approach may be difficult to implement because induction heating is usually accomplished by resonant circuits that generate high currents. The resonant circuit and high-frequency signal injection must be kept from interfering with or destroying the normal stator-coil driver electronics. The preheating cycle would not be functioning during normal motor levitation and rotation. 


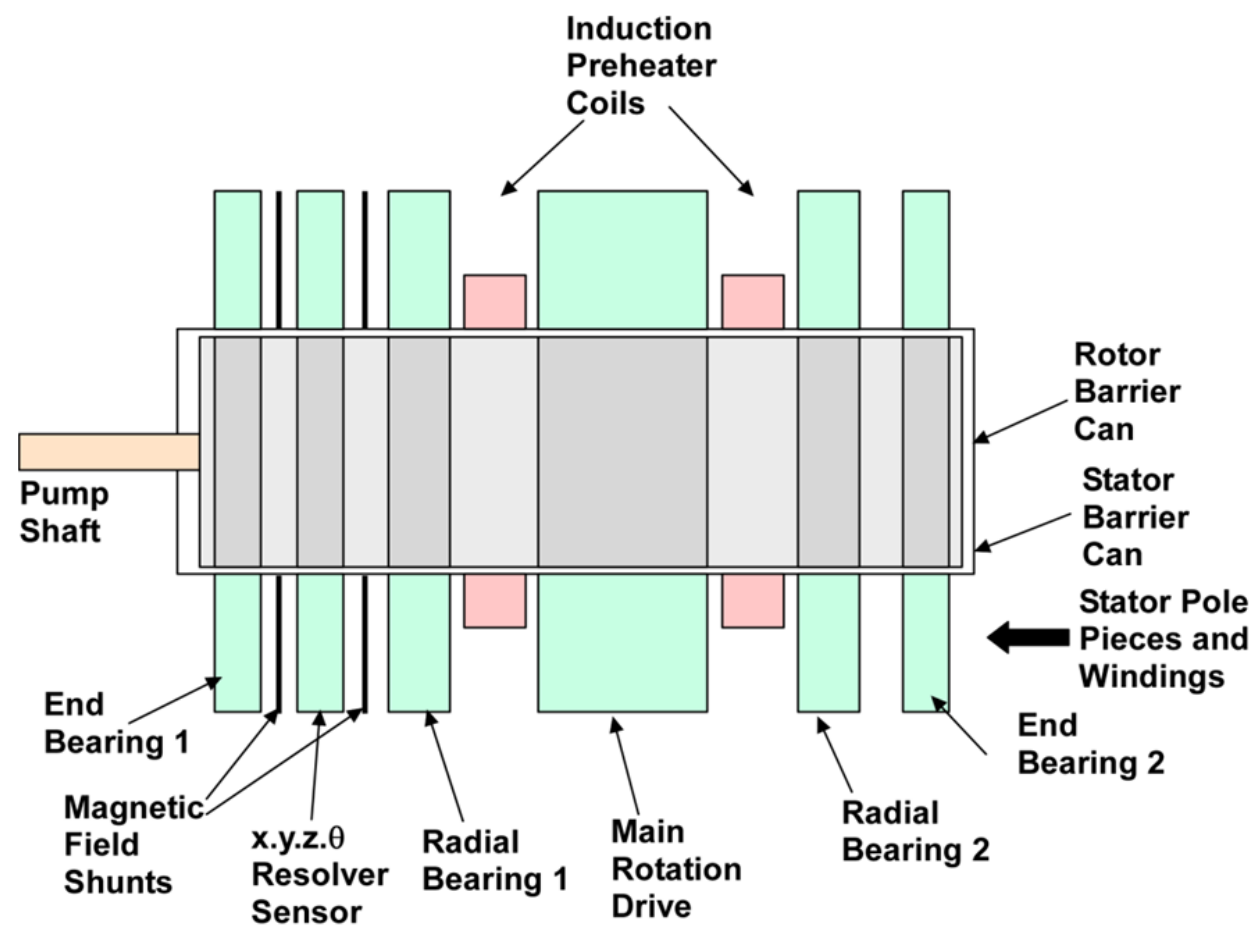

Fig. 88. Preheating system induction concept illustration.

\subsection{MATERIALS SELECTION}

The near-term selected materials for various parts of the demonstration system are presented in Appendix A. In some cases, for example, for the cans, a material, Hastelloy ${ }^{\mathbb{R}} \mathrm{N}$, has been uniquely identified, as it possesses the corrosion resistance, supplies the needed mechanical properties, and displays the necessary manufacturing characteristics. In other cases, alternative materials are presented because the individual material possesses some of the needed characteristics such as corrosion resistance but the mechanical properties at temperature would result in significantly large cross-sectional components. The alternative material may demonstrate better mechanical properties, but the corrosion resistance is being inferred at this time. An example of a component that falls into this category is the pump shaft for which Hastelloy ${ }^{\circledR}$ N, Hastelloy ${ }^{\circledR} 600$, and Haynes ${ }^{\circledR} 214$ are shown as possible materials of construction. While Hiperco ${ }^{\circledR} 50$ has initially been chosen as the material of construction of the rotor and stator laminations because of stable magnetic properties at temperature and reasonable mechanical properties, there is uncertainty as to which of a handful of high-temperature ceramics will be employed for the insulation between laminates. Similar uncertainty remains for the insulation for the motor windings. Other components and their associated choices of materials and possible materials issues are presented in Appendix A. The stack of component materials in the motor is shown in Fig. 64.

\subsection{TESTING PLAN}

Testing is performed to investigate technical details, verify sub-systems, and to verify the system. System performance is an integration of equipment that must meet the system specification and be incorporated in the various subsystem specifications and requirements. ${ }^{58}$ Testing considerations include the following.

1. Harsh environments such as temperature and corrosive materials such as molten salt

2. Performance of structural, mechanical, electrical, magnetic, sensing, electronics, computation, and software sub-system elements of the system 
3. Function of structural, mechanical, electrical, magnetic, sensing, electronics, computation, and software sub-system elements of the system

4. Failure modes, reliability, and the associated detection and mitigation of failure events

Typically, the testing is aligned with the technical design progress to address the needs identified in the design phase and later to test sub-systems and systems as the design progress to test articles. Figure 89 illustrates the test progression to support early design activities and later subsystem testing.

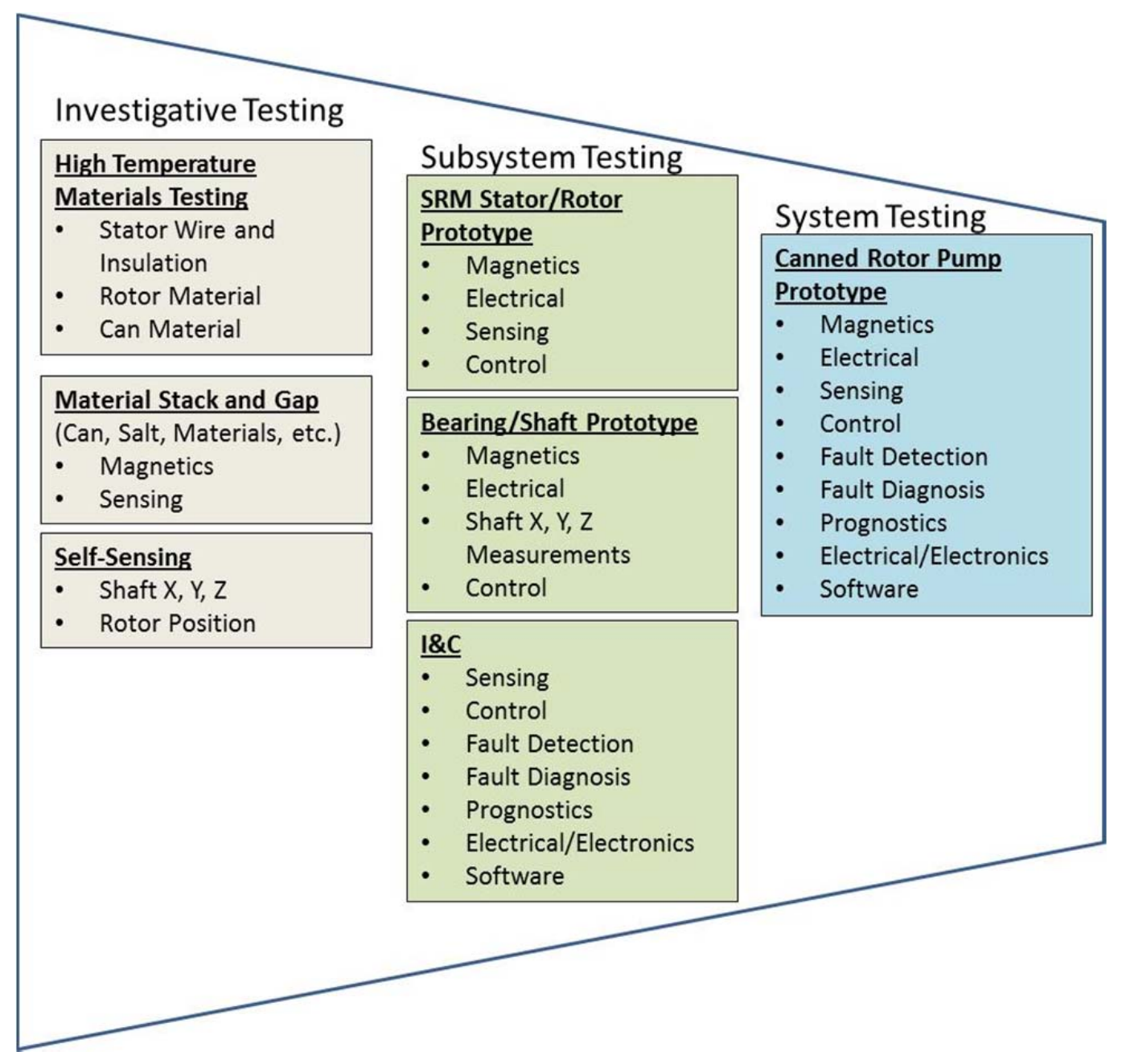

Fig. 89. Illustration of test progression.

This testing may occur in various laboratory facilities based on the test requirements. Testing may require manipulated environment condition (temperature), electrical infrastructure, I\&C infrastructure, and other supporting capabilities. Control, fault detection, prognostics, and software testing may occur in both simulation environments and on prototypical test articles.

\section{CONCLUSIONS AND NEXT STEPS}

Embedding modern sensors and controls deeply within the SSC of NPPs has the potential to provide improved performance and reliability, as well as reducing the maintenance cost. Work to date on the embedded sensors and control project has produced a conceptual design that translates the requirements 
for a high-temperature, harsh-environment pump into a concept for physical layout, as well as outlining the required sensors, controls, and materials.

Developing and demonstrating a canned-rotor, magnetic bearing pump suitable for a high-temperature, liquid fluoride salt environment is a complex, interdisciplinary task. This report documents the underlying theory necessary to create a conceptually viable integrated system design. A strategy for embedding I\&C within the design has also been developed. The strategy and theory sections provide a basis for the performance and design requirements that are also described extensively. The conceptual design represents the outgrowth of those engineering investigations. Overall, while a significant amount of research, development, and demonstration remains, the basic concept viability is reasonably ensured by the assessment and modeling performed thus far.

The hardware development objective of the project is to design, construct, and demonstrate a pump system that achieves improved operational performance and reliability due to the inclusion of deeply embedded I\&C. The conceptual design is the first step in describing a constructible pump system having embedded sensors and controls to accomplish that objective.

The rate of progress will slow over the next year in response to reduced 2013 funding. Consequently, only limited design advancement will be possible. The two major tasks for the next year will be creation of an integrated performance model and an assessment of potential fabrication methods and assembly techniques. Development of an integrated modeling and simulation capability allows predication of the assembled device performance and provides a platform to assess the impact of design changes. The integrated design model will also enable assessment of how specific design features accomplish design objectives. The pump must be designed to gracefully degrade in the event of unplanned subcomponent failures and allow for on-line exterior subcomponent replacement. Moreover, the embedded measurements need to be able to identify impending failures to allow any necessary maintenance to be performed during planned plant shutdowns. The integrated model will include consideration of both the embedded measurements and system degradation over time.

Assessment of the fabrication methods and system assembly techniques is necessary to allow component fabrication and testing to begin in the following year. The current conceptual design was based upon creating a conceptually reasonable system and did not place major emphasis on how all of the subcomponents will be constructed and then assembled into a working device. As design matures the details of how the system will be assembled and what will be the fabrication sequence need to be resolved. Moreover, ensuring that the lower temperature and surrogate material testing planned for the following year generates useful data to support the final system requires both an integrated model of the final system as well as a general understanding of the material and construction details of the eventual high-temperature system. 


\section{REFERENCES}

1. ANSI/EIA-632-1998, Processes for Engineering a System, Washington, D.C, Electronic Industries Association (EIA), 1999.

2. Hall, M. N. "Reducing Longterm System Cost by Expanding the Role of the Systems Engineer," Proceedings of the 1993 International Symposium on Technology and Society, IEEE, Washington DC, 1993, pp. 151-155.

3. Miller, R., Floricel, S., Lessard, D.R., The Strategic Management of Large Engineering Projects, MIT Press, 2000.

4. M.M. Bakran and H.-G. Eckel, "Power Electronics Technologies for Locomotives," IEEE Power Conversion Conference - Nagoya, 2007. PCC '07.

5. "Davis-Besse Reactor Pressure Vessel Head Degradation: Overview," NUREG/BR-0353, Rev. 1, Aug. 2008.

6. Kurt A. Polzin, "Liquid Metal Pump Technologies for Nuclear Surface Power," Proceedings of Space Nuclear Conference 2007, Boston, Massachusetts, June 24-28 2007, Paper 2002.

7. A. Chiba, T. Fukao, O. Ichikawa, M. Oshima, M. Takemoto, and D. G. Dorrell, Magnetic Bearings and Bearingless Drives, Elsevier, 2005

8. Praveen Vijayraghavan, Design of Switched Reluctance Motors and Development of a Universal Controller for Switched Reluctance and Permanent Magnet Brushless DC Motor Drives, PhD Dissertation, Virginia Polytechnic Institute and State University, November 2001

9. James E. Tomayko. "Computers Take Flight: A History of NASA's Pioneering Digital Fly-By-Wire Project," NASA-SP-2000-4224. [Online\}, 2000. Available at http://www.nasa.gov/centers/dryden/pdf/182985main_DFBW_rev1.pdf.

10. The Impact of Control Technology, Overview, Success Stories, and Research Challenges, IEEE Control System Society, February 2011, http://ieeecss.org/main/IoCT-report.

11. Studebaker, P., "The Champion of Dynamic Matrix Control", Control Engineer Magazine, March 1998, Vol. Xi, No. 03, pp. 34-38.

12. Dynamic Controller, Frustrated by Conventional Advanced Control Techniques, Charlie Cutler Had to Find a Better Way, ControlGlobal.com, May 01, 2003, http://www.controlglobal.com/articles/2003/159.html?page=print

13. Miller, T.J.E., Electronic Control of Switched Reluctance Machines, Elsevier Newnews, pp. 28-31, 2008

14. Krishnan, R., Switched Reluctance Motor Drives, CRC Press, 2001, pp. 388-392.

15. Chiba, A., Fukao, T., Ichikawa, O., Oshima, M., Takemoto, M., and Dorrel, D.G., Magnetic Bearings and Bearingless Drives, Elsevier Newnews, 2008.

16. C. P. Colker, C. S. Mah, and C. L. Foss, "Design and Development of a Canned-Motor Pump for High Temperature NaK Service in SNAP-8," NASA CR-72823 (Aerojet 3663), NASA Lewis Research Center, April 1971.

17. P. E. Kueser, et. al. (Westinghouse Electric Corporation), "Electrical Conductors and Electrical Insulation Materials Topical Report," NASA CR-54092 (Westinghouse 64.53E), Lewis Research Center (Oct, 1964), 777 pages.

18. P. E. Kueser, et. al., (Westinghouse Electric Corporation), "Magnetic Materials Topical Report Research and Development Program on Magnetic, Electrical Conductor, Electrical Insulation, and Bore Seal Materials," NASA CR-54091 (Westinghouse 64.52E) Lewis Research Center (Sept., 1964) 628 pages.

19. P. E. Kueser, et. al. (Westinghouse Electric Corporation), "Properties of Magnetic Materials for Use in High-Temperature Space Power Systems," NASA SP-3043, Lewis Research Center (1967) 328 pages. 
20. P. E. Kueser, et. al. (Westinghouse Electric Corporation), "High Temperature (800 to 1600 F) Magnetic Materials," NASA CR-1460, Lewis Research Center (March, 1970) 342 pages.

21. W. L. Grant and P. E. Kueser (Westinghouse Electric Corporation), "Thermal Vacuum Testing of High Temperature Electrical Components," NASA CR-1592, Lewis Research Center (Aug, 1970), 265 pages.

22. Jerr Ji, "High Temperature Mechanisms - A Breakthrough Development," , 6th International Planetary Probe Workshop, Atlanta, Georgia (6/21-22/2008) [Available at http://smartech.gatech.edu/handle/1853/26384?show=full]

23. G. Montague, et. al., "Design and Fabrication of High-Temperature, Radial Magnetic Bearing for Turbomachinery," NASA/TM-2003-212300, July 2003.

24. A. J. Provenza, et. al., "High Temperature Characterization of a Radial Magnetic Bearing for Turbomachinery," Transactions of the ASME, Vol. 127, April 2005, pp. 437-444.

25. G. Montague, et. al., "High-Temperature Switched-Reluctance Electric Motor," NASA Tech Briefs, February 2003, p. 39.

26. R. Moore et al., "Design Considerations for Concentrating Solar Power Tower Systems Employing Molten Salt," Sandia Report SAND2010-6978, Sept. 2010.

27 P. G. Smith, Experience with High-Temperature Centrifugal Pumps in Nuclear Reactors and Their Application to Molten-Salt Thermal Breeder Reactors, ORNL/TM-1993, Oak Ridge National Laboratory, Oak Ridge, Tennessee, 1967.

28 J. L. Crowley, W. B. McDonald, and D. L. Clark, Design and Operation of Forced-Circulation Corrosion Testing Loops with Molten Salt, ORNL/TM-528, Oak Ridge National Laboratory, Oak Ridge, Tennessee. 1963.

29 J. W. Kroger, A Forced-Circulation Loop for Corrosion Studies: Hastelloy N Compatibility with $\mathrm{NaBF}_{4}-\mathrm{NaF}$ (92.8 mole \%), ORNL/TM-4221, Oak Ridge National Laboratory, Oak Ridge, Tennessee, 1972.

30 R. C. Robertson; MSRE Design and Operations Report. Part I. Description of Reactor Design, ORNL/TM-728, Oak Ridge National Laboratory, Oak Ridge, Tennessee. 1965.

31 P. G. Smith,_Development of Fuel-and Coolant-Salt Centrifugal Pumps for the Molten-Salt Reactor Experiment, ORNL/TM-2987, Oak Ridge National Laboratory, Oak Ridge, Tennessee. 1970.

32 J. R. Engel, P. N. Haubenreich, and A. Houtzeel, Spray, Mist, Bubbles, and Foam in the Molten Salt Reactor Experiment,_ORNL/TM-3027, Oak Ridge National Laboratory, Oak Ridge, Tennessee. 1970.

33. G. Yoder et al., "Development of a Forced-Convection Liquid-Fluoride-Salt Test Loop," Proceedings of HTR 2010, Prague, Czech Republic, October 18-20, 2010, Paper 197.

34. A.V. Belevstev, A.V. Karzhavin, and A.A. Ulanowsky, Stability of a Cable Nicrosil-Nisil Thermocouple Under Thermal Cycling, Temperature: Its Measurement and Control in Science and Industry, Volume 7, edited by Dean C. Ripple, pp. 453-7 (2003).

35. Jeff Jablin, Michael R. Storar, and Phillip L. Gray, Improved Operating Efficiency Through the Use of Stabilized Thermocouples, Journal of Engineering for Gas Turbines and Power, 122, pp. 659-63 (October 2000).

36. N. A. Burley, Advanced Integrally Sheathed Type N Thermocouple of Ultra-High Thermoelectric Stability, Measurement, 8(1), pp. 36-41 (Jan-Mar 1990).

37. Procedures for Performing a Failure Mode, Effects and Critically Analysis. Rep. no. MIL-STD1629A. Washington DC: Department of Defense, 1977.

38. Mraz, Miha, and Bernhard Huber. FMEA - FMECA. Rep. Ljubljana: University of Ljubljana, 2005.

39. Hughes, A., Electric Motors and Drives, Third Edition, Elsevier Newnes, 2011.

40. Schweitzer, G. and Maslen, E. H., ed., "Magnetic Bearings: Theory, Design, and Application to Rotating Machinery," Springer, 2010.

41. Zhou, K., Doyle, J. C., and Glover, K., "Robust and Optimal Control”, Prentice Hall, 1996. 
42. Cummins, W., Corletti, M., Schulz, T., Westinghouse Electric Company, LLC, Westinghouse AP1000 Advanced Passive Plant, Proceedings of ICAPP '03, Cordoba, Spain, May 4-7, 2003, Paper 3235.

43. Y. Katoh et al., "Assessment of Silicon Carbide Composites for Advanced Salt Cooled Reactors," ORNL Report ORNL/TM-2007/168, Sept. 2007.

44. D. W. McKee and D. Chatterji, "Corrosion of Silicon Carbide in Gases and Alkaline Melts," Journal of The American Ceramic Society, 59,(9-10) 441-444 (1976).

45. Richard T. Fingers, "Creep Behavior of Thin Laminates of Iron Cobalt Alloys for Use in Switched Reluctance Motors and Generators", AFRL-PR-WP-TR-199-2053, June 1999, Propulsion Directorate Air Force Research Laboratory, Air Force Materiel Command, Wright-Patterson AFB OH 4533-7251.

46. http://www.specialmetals.com/documents/Inconel alloy 600 (Sept 2008).pdf

47. D. Holcomb and S. Cetiner, "An Overview of Liquid-Fluoride-Salt Heat Transport Systems," ORNL/TM-2010/156, Sept. 2010.

48. Henley, E.J., Kumamoto, H., Reliability Engineering and Risk Assessment, Prentice-Hall, Inc., 1981.

49. Ellis, D.O., Ludwig, F.J., Systems Philosophy, Prentice-Hall, Inc. 1962.

50. Baer-Riedhart, J., Digital Electronic Engine Control Fault Detection and Accomodation Flight Evaluation, NASA Ames Research Center Archives, DFRF83-614, http://ntrs.nasa.gov/archive/nasa/casi.ntrs.nasa.gov/19860015878_1986015878.pdf.

51. Boehm, J., Gerber, R., and Kiely, N.R.C., "Sensors for Magnetic Bearings", IEEE Transactions on Magnetics, Vol. 29, No. 6, November 1993, pp.2962-2964.

52. Kaman, Inductive Technology Handbook, www.kamansensors.com.

53. Mukhopadhyay, S.C, "Do we really need sensors? A Sensorless Magnetic Bearing Perspective," 1st International Conference on Sensing Technology November 21-23, 2005, Palmerston, North, New Zealand, pp. 425-431.

54. Park, Y.H., Han, D.C., Park, I.H, , Ahn, H.J., and Jang, D.Y., "A self-sensing technology of active magnetic bearings using a phase modulation algorithm based on a high frequency voltage injection method", Journal of Mechanical Science and Technology 22 (2008), pp. 1757-1764.

55. Chiba, A., Fukao, T., Ichikawa, O., Oshima, M., Takemoto, M., and Dorrel, D.G., Magnetic Bearings and Bearingless Drives, Elsevier Newnews, 2008.

56. Krishnan, R., Switched Reluctance Motor Drives, CRC Press, 2001, page 237.

57. Miller, T.J.E., Electronic Control of Switched Reluctance Machines, Newnews, 2001.

58. Machol, R.E., Tanner, W.P., Alexander, S.N., System Engineering Handbook, McGraw-Hill Book Company, 1965. 



\section{APPENDIX A}

\section{A.1 MECHANICAL DESIGN}

\section{A.1.1 Mass and Motor Mounting}

An estimate of the motor mass is needed for the inertial energy calculations that are a part of control system design. Table A-1 lists the expected masses of various motor components.

Table A-1. Approximate expected mass of the system

\begin{tabular}{ll}
\hline \multicolumn{1}{c}{ Component } & \multicolumn{1}{c}{ Approximate expected mass } \\
\hline Axle & $8.1 \mathrm{~kg}>\mathrm{m}>7.45 \mathrm{~kg}$ for $0.5 \mathrm{~cm}<\mathrm{d}<1.5$ \\
& $\mathrm{~cm}$ \\
Rotor & $3.5 \mathrm{~kg}$ \\
Stator & $5.9 \mathrm{~kg}$ \\
Inner Bearing & $3.1 \mathrm{~kg}$ \\
Outer Bearing & $4.5 \mathrm{~kg}$ \\
Complete System & $80 \mathrm{~kg}$ \\
\hline
\end{tabular}

\section{Mounting}

The mounting structure that supports the canned rotor pump must fulfill several requirements regarding structural rigidity, temperature exposure and system flexibility. Some of the considerations and conditions of the support structure are outlined in Table A-2.

Table A-2. Structural rigidity factors

\begin{tabular}{l|l}
\hline Weight & Must be able to support the pump weight for a period of seven years \\
Disturbance Forces & Must be designed to withstand unexpected disturbance forces. \\
Vibration & Must be able to isolate the adjacent equipment from any vibrations \\
Stiffness & Must be able to prevent the maximum allowed deflection \\
Temperature & Must withstand the operating temperatures of $600-750^{\circ} \mathrm{C}$ \\
Flexibility & $\begin{array}{l}\text { Must allow for adjustability in x-y-z directions in order to adapt to the } \\
\text { particular setups in different reactor equipment }\end{array}$ \\
\hline
\end{tabular}

\section{A.1.2 Size of Impeller, Shaft, and Pump Volute}

The physical sizes of the pump components are indicated. Table A-3 lists the impeller dimensions. Table A-4 shows the pump body. Table A-5 comments on design factors associated with the shaft. 
Table A-3. Impeller component dimensions

\begin{tabular}{lccc}
\hline \multicolumn{1}{c}{ Impeller } & Dim. & Unit \\
\hline \multirow{2}{*}{ Outer Diameter } & Disc & \\
Inner Diameter & \multicolumn{1}{c}{14.29} & $\mathrm{~cm}$ \\
Thickness & 2.54 & $\mathrm{~cm}$ \\
\multicolumn{2}{c}{1.27} & $\mathrm{~cm}$ \\
Outer Diameter & Extrusion & \\
Thickness & 3.81 & $\mathrm{~cm}$ \\
Inner Diameter & 1.27 & $\mathrm{~cm}$ \\
& 2.54 & $\mathrm{~cm}$ \\
Thickness & Vanes & \\
Total Impeller Thickness & 6.99 & $\mathrm{~cm}$ \\
\hline
\end{tabular}

Table A-4. Pump body dimensions

\begin{tabular}{lcc}
\hline \multicolumn{1}{c}{ Pump Body } & Dim. & Unit \\
\hline Inlet Diameter & 5.08 & $\mathrm{~cm}$ \\
Outlet Diameter & 6.35 & $\mathrm{~cm}$ \\
\hline
\end{tabular}

Table A-5. Shaft dimensioning factors

\begin{tabular}{ll}
\hline \multicolumn{1}{c}{ Dimension } & \multicolumn{1}{c}{ Considerations } \\
\hline Outer Diameter & $\begin{array}{l}\text { - Will be joined to the inner diameter of the rotor, the radial } \\
\text { bearings, and the spacers. } \\
\text { - Must allow torque transmission from rotor to impeller. } \\
\text { Inner Diameter }\end{array}$ \\
$\begin{array}{l}\text { Should be designed for optimal flow for cooling. See Section 5.2.1. } \\
\text { Length }\end{array}$ & $\begin{array}{l}\text { Will be joined to impeller inner diameter: see Table 19. } \\
\text { Should accommodate rotor, as well as axial and radial bearings. }\end{array}$ \\
\hline
\end{tabular}

\section{A.1.3 Inertia}

To determine optimal design decisions and optimize control algorithms, it is useful to estimate the polar moment of inertia of the various components of the canned-rotor system. Table A- 6 outlines each component and its corresponding polar moment of inertia. 
Table A-6. Estimates of polar moments of inertia

\begin{tabular}{lll}
\hline \multicolumn{1}{c}{ Component } & \multicolumn{1}{c}{ Dimensions } & \multicolumn{1}{c}{$\begin{array}{c}\text { Approximate Moment of } \\
\text { Inertia }\left(\mathbf{k g}^{2} \mathbf{c m}^{2}\right)\end{array}$} \\
\hline Axle & Length=46.4 cm & $100.2<\mathrm{I}<99.9$ \\
& Shaft Outer Diameter $=4.1 \mathrm{~cm}$ & $8.1 \mathrm{~kg}>\mathrm{m}>7.45 \mathrm{~kg}$ \\
& Axial bearing disc outer diameter $=12.7 \mathrm{~cm}$ & \\
& Shaft Inner Diameter: & \\
& $0.5 \mathrm{~cm}<\mathrm{d}<1.5 \mathrm{~cm}$ & $\mathrm{I}=47.9$ \\
Rotor Laminations & Inner Diameter: $4 \mathrm{~cm}$ & \\
& Outer diameter: $8 \mathrm{~cm}$ & \\
& Rotor Pole Area: $2 \mathrm{~cm} \times 2 \mathrm{~cm}$ & $\mathrm{I}=520.5$ \\
& Length: $7.6 \mathrm{~cm}$ & \\
Stator & Inner Radius: $8.9 \mathrm{~cm}$ & \\
& Outer Radius: $10.4 \mathrm{~cm}$ & $\mathrm{I}=5314.12$ \\
& Stator Pole: $2.1 \mathrm{~cm} \times 2.3 \mathrm{~cm}$ & \\
\hline \multirow{2}{*}{ Complete System } & Total Length: $53.5 \mathrm{~cm}$ & \\
& Outer Structure Diameter: $22.3 \mathrm{~cm}$ & \\
\hline
\end{tabular}

\section{A.1.4 Stiffness}

To estimate shaft stiffness, the shaft has been modeled as a stationary simply supported beam under the load of the rotor weight. For this initial model, the bearings will be modeled as rigid. The Jeffcott rotor model, which consists of an elastic shaft and a rigid disk, with the bearings serving as supports, will be used. An illustration of this model, as well as a representation of its maximum deflection, is shown in Fig. A-1.
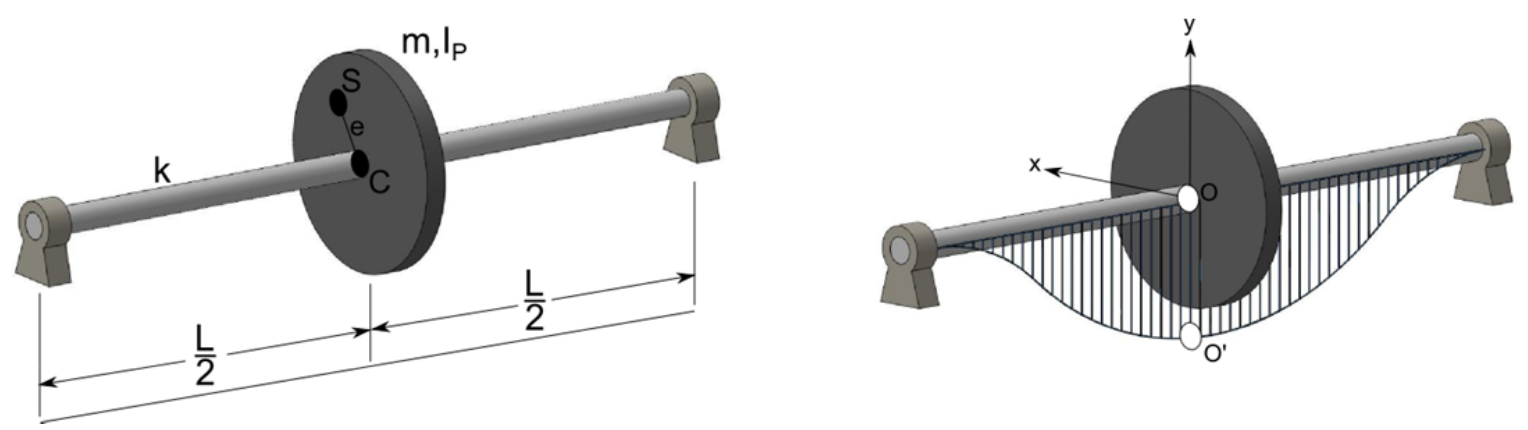

Fig. A-1. Simple bearing model for estimating shaft deflection.

Assuming a rotor mass of $232 \mathrm{~N}$ (estimate from current preliminary design), the required shaft stiffness for a range of maximum deflections is shown in Fig. A-2. 


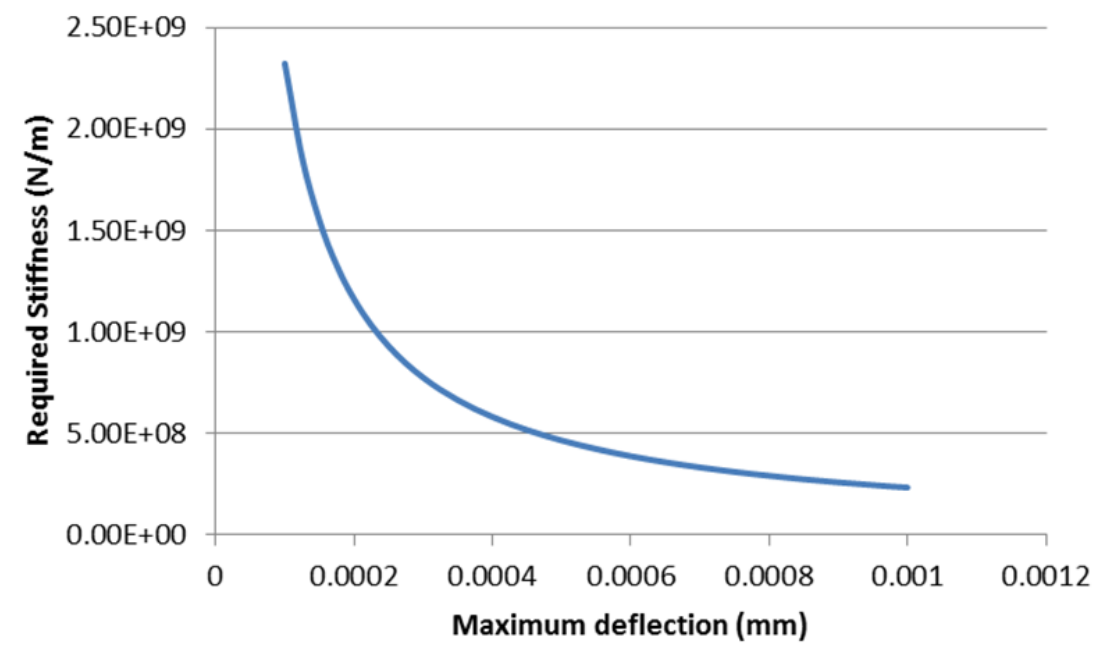

Fig. A-2. Required stiffness as a function of maximum deflection.

Given preliminary dimensions for length and shaft outer diameter, and taking into account both the rotor and the impeller weight, estimated deflections can be found at both the center of the shaft, and the impeller end. Varying the inner diameter of the shaft, the graphs, Figs. A-3 and A-4, show the estimated deflections versus rod inner diameter.

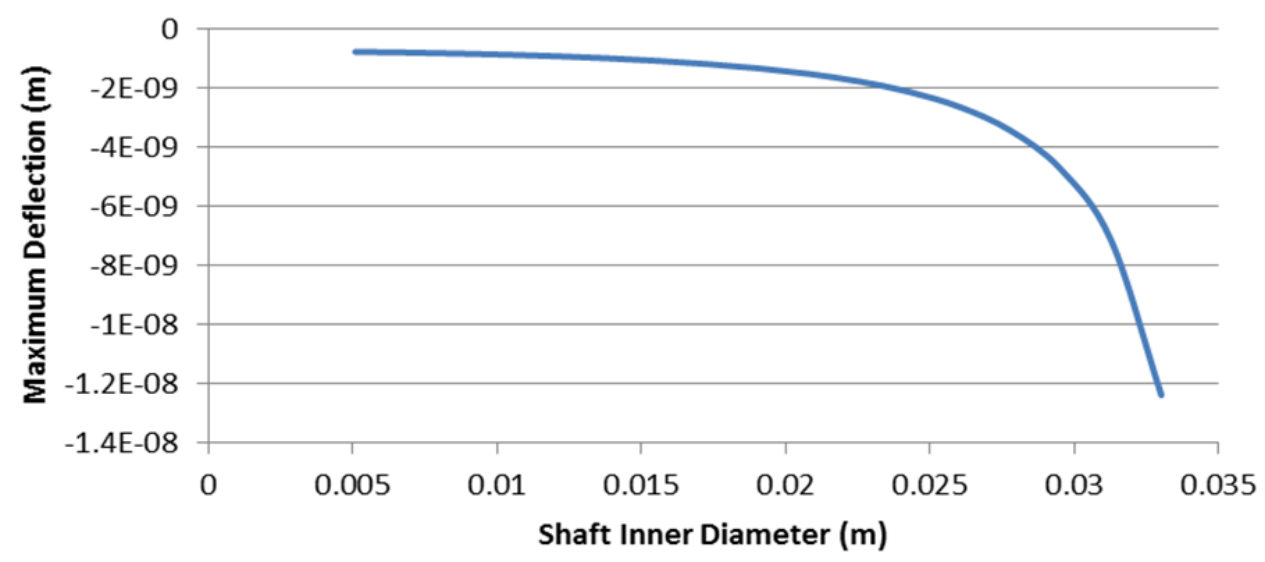

Fig. A-3. Deflection at center of shaft. 


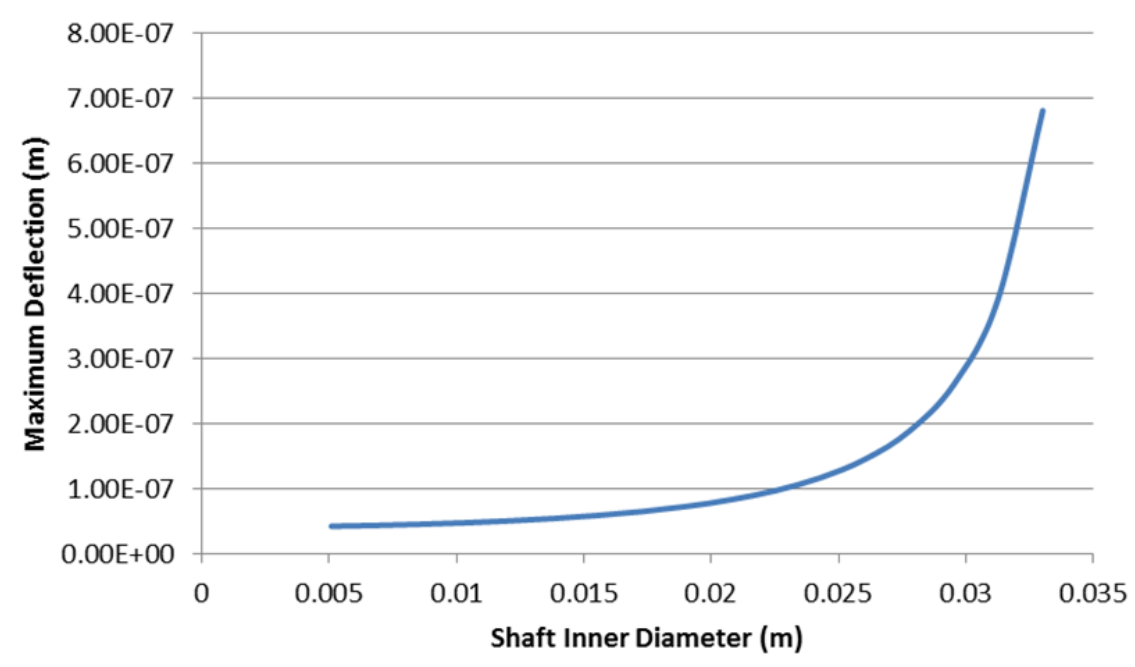

Fig. A-4. Deflection at impeller end.

\section{Elastic Bearings}

The stiffness contribution of the bearings can also be taken into account once they are modeled as elastic. In this case, bearings may be modeled as elastic members, and their respective stiffness work in parallel, while they are in series. The resulting stiffness in both the $\mathrm{x}$ and $\mathrm{y}$ direction is shown in Fig. A-5 and represented by Equation(s) (85).
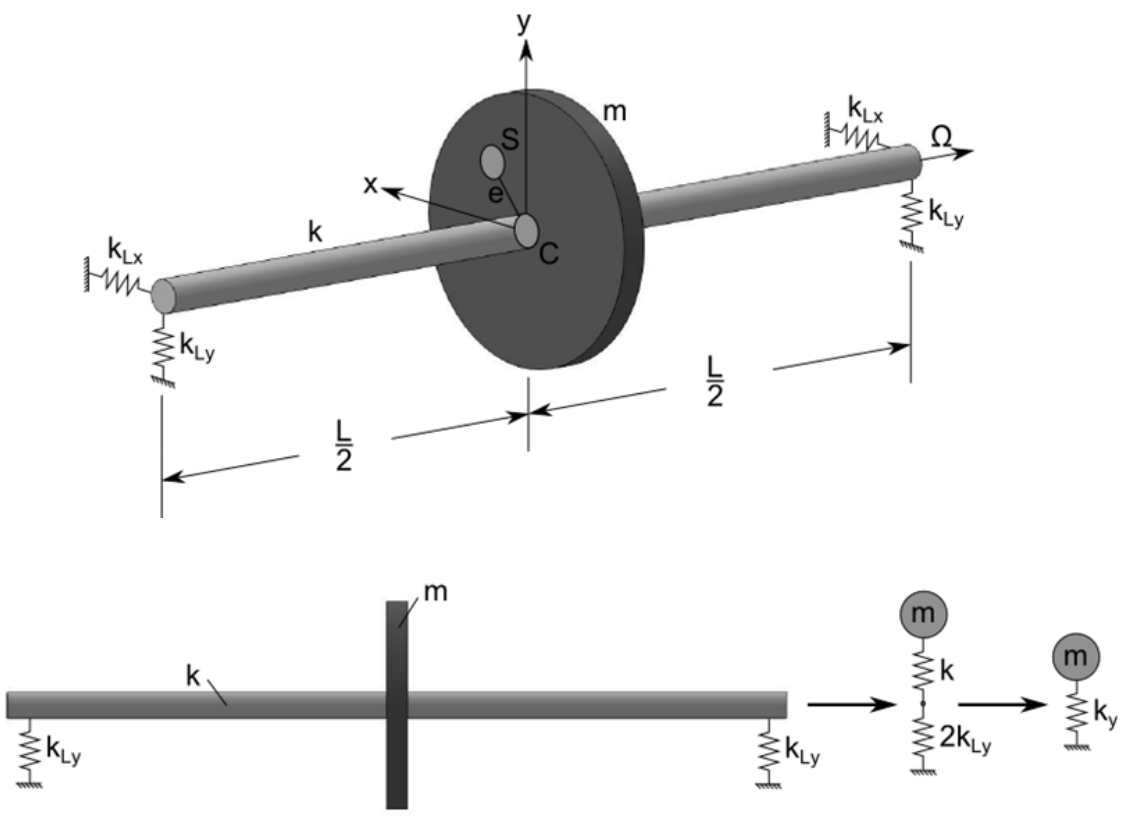

Fig. A-5. Modeling of bearings as elastic members. 


$$
\begin{aligned}
& k_{\chi}=\frac{2 k k_{L \chi}}{k+2 k_{L \chi}} \\
& k_{y}=\frac{2 k k_{L y}}{k+2 k_{L Y}}
\end{aligned}
$$

As there will be control of the forces provided by the magnetic bearings, the bearing stiffness may be modified to fulfill requirements.

\section{A.1.5 Natural Frequency, Balance and Eccentricity Specification}

The fundamental, undamped natural frequency of the reactor coolant pump rotating assembly is calculated for simple supports at the bearing locations and the rotor vibrating in air. This frequency, defined as the "classical lateral critical speed" should be greater than 125 percent of the normal operating speed.

Determination of the damped natural frequency of the reactor-coolant pump rotor bearing system model includes the effects of the bearing films, can annular fluid interaction, motor magnetic phenomena, and pump structure. The damped natural frequencies for the AP1000 canned-motor pump exhibit sufficient energy dissipation to be stable. The high degree of damping provides smooth pump operation.

The pump is analyzed for the response of the rotor and stator to external forcing functions. The support and connection of the pump to the steam generator and piping are considered in the analysis. The responses are evaluated using criteria including critical loads, stress deformation, wear, and displacement limits to establish the actual system critical speeds.

As a preliminary estimation, the shaft is modeled as a beam under an evenly distributed load of the rotor and the impeller. The shaft natural frequency can be described as Equation (86):

$$
\omega=\sqrt{\frac{k}{m}},
$$

where $\mathrm{k}$ refers to the beam stiffness, and $\mathrm{m}$ to the shaft mass.

Given dimensions for the shaft length and outer diameter, an estimate of the natural frequency of the shaft vs. shaft inner diameter is shown in Fig. A-6. These frequencies are not in close proximity to the limiting frequencies of the motor drive. 


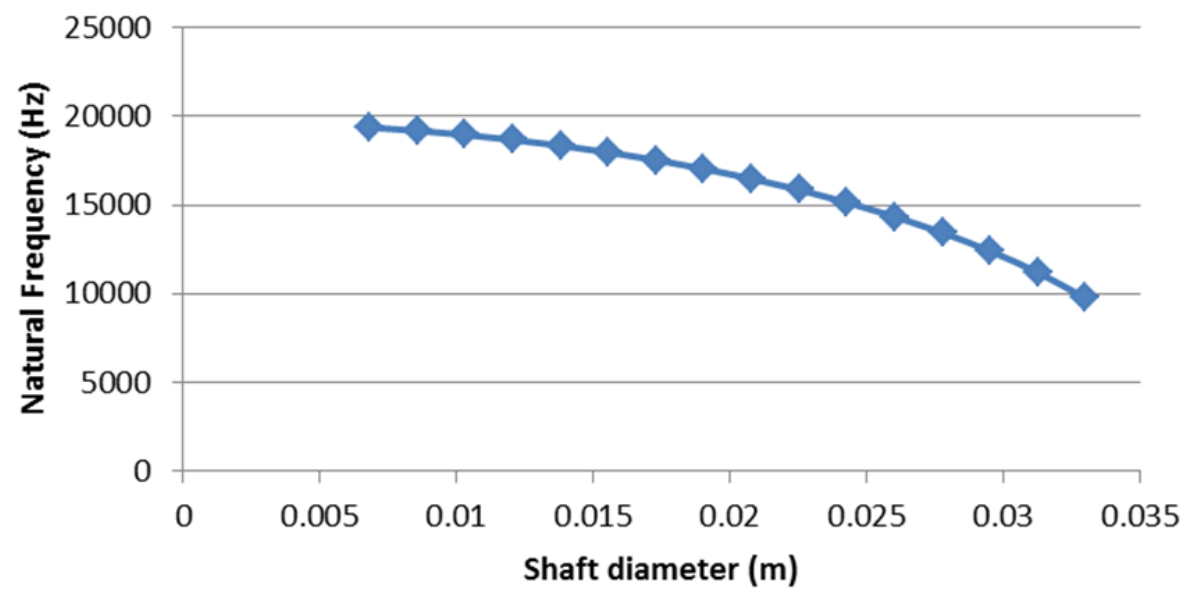

Fig. A-6. Shaft resonant frequency as a function of diameter.

Assuming elastic bearings, the stiffness $\mathrm{k}_{\mathrm{LX}}$ and $\mathrm{k}_{\mathrm{LY}}$ of the bearings must be taken into account. In this case, the $\mathrm{x}$ and $\mathrm{y}$ natural frequency become the following Equation(s) (87):

$$
\begin{aligned}
& \omega_{y}=\omega \sqrt{\frac{1}{1+\frac{k}{2 k_{L Y}}}} \\
& \omega_{\chi}=\omega \sqrt{\frac{1}{1+\frac{k}{2 k_{L \chi}}}}
\end{aligned}
$$

\section{A.1.6 Fabrication and Assembly Design Restrictions}

This section captures material selections for various motor components and alternative machining and fabrication techniques (see Table A-7). Table A-8 suggests assembly methods and identifies some assembly issues. 
Table A-7. Motor materials and tolerances

\begin{tabular}{|c|c|c|c|}
\hline Component & Material & $\begin{array}{c}\text { Potential manufacturing } \\
\text { processes }\end{array}$ & $\begin{array}{l}\text { Fabrication } \\
\text { Considerations }\end{array}$ \\
\hline Axle & Inconel $^{\circledR} 600$ & $\begin{array}{l}\text { Turning /Extrusion /rolling } \\
\text { (spline) } \\
\text { Post machining } \\
\text { Welding (of disc) }\end{array}$ & \\
\hline Rotor Laminations & Vacodur $^{\circledR} 50 /$ Hiperco50 & $\begin{array}{l}\text { Laser Cut/Stamping/ } \\
\text { Waterjet/Wire EDM } \\
\text { Welding for joining laminations }\end{array}$ & $\begin{array}{l}\text { Effects of high } \\
\text { temperatures on welded } \\
\text { joints }\end{array}$ \\
\hline Rotor Can & Hastelloy ${ }^{\circledR} \mathrm{N}$ & $\begin{array}{l}\text { Machining } \\
\text { Welding (endcap) }\end{array}$ & $\begin{array}{l}\text { Tolerances in the order of } \\
0.001 \text { in } \\
\text { Good Surface Finish } \\
\text { May need to post-machine } \\
\text { with rotor. }\end{array}$ \\
\hline Catcher Bearings & Graphite/ Silicon Carbide & & \\
\hline Stator Laminations & Hiperco $^{\circledR} 50$ & $\begin{array}{l}\text { Laser Cut/Stamping/ } \\
\text { Waterjet/Wire EDM } \\
\text { Welding for joining laminations }\end{array}$ & \\
\hline Stator Can & Hastelloy $^{\circledR} \mathrm{N}$ & Machining & $\begin{array}{l}\text { Tolerances in the order of } \\
0.001 \text { in } \\
\text { Good Surface Finish }\end{array}$ \\
\hline Structural Cylinder & Steel & $\begin{array}{l}\text { Spline: Bolted joints } \\
\text { Cast }\end{array}$ & \\
\hline
\end{tabular}

Table A-8. Rotor assembly issues

\begin{tabular}{|c|c|c|}
\hline Components & $\begin{array}{l}\text { Potential Assembly } \\
\text { Methods }\end{array}$ & Considerations \\
\hline $\begin{array}{l}\text { Rotor, radial bearings, } \\
\text { spacers and shaft }\end{array}$ & $\begin{array}{l}\text { Welding } \\
\text { Bolted Joints } \\
\text { Press-fit }\end{array}$ & $\begin{array}{l}\text { Any fasteners used must be able to withstand the operating } \\
\text { temperatures. } \\
\text { Fasteners must maintain desired torque even if not stationary. } \\
\text { Due to thermal expansion, the interference fit might change after } \\
\text { temperature rise. } \\
\text { Thermal expansion might cause components to slide in the shaft. }\end{array}$ \\
\hline Axial bearing and shaft & Press fit/Welding & $\begin{array}{l}\text { Due to thermal expansion, an interference fit might change after } \\
\text { temperature rise. } \\
\text { Welding would require reaching the back surface of the bearing. }\end{array}$ \\
\hline $\begin{array}{l}\text { Ceramic potting and } \\
\text { rotor }\end{array}$ & Pour and cure & $\begin{array}{l}\text { Lamination insulators may be inserted as sheets or may be poured } \\
\text { in place and baked to cure. }\end{array}$ \\
\hline $\begin{array}{l}\text { Inner rotor system and } \\
\text { rotor can }\end{array}$ & Welding & There must be no leakage between the rotor system-can interface. \\
\hline Catcher bearings & Press fit/Weld & Surface must be clean for smooth contact with rotor can. \\
\hline $\begin{array}{l}\text { Stator Can and Stator } \\
\text { system }\end{array}$ & Flexitallic gasket & $\begin{array}{l}\text { There must be no leakage between the stator system-can } \\
\text { interface. }\end{array}$ \\
\hline $\begin{array}{l}\text { Stator system and outer } \\
\text { structure }\end{array}$ & Flexitallic Gasket & \\
\hline
\end{tabular}


See Table A-9 for materials descriptions.

Table A-9. List of components and materials

\begin{tabular}{|c|c|c|c|c|c|c|c|c|c|c|}
\hline \multirow[b]{3}{*}{ Component } & \multirow[b]{3}{*}{ Subcomponent } & \multicolumn{8}{|c|}{ Material } & \multirow[b]{3}{*}{ Comments } \\
\hline & & & & & & & & Expected operating & & \\
\hline & & Material & Max use temp $\left({ }^{\circ} \mathrm{C}\right)$ & $\begin{array}{c}\text { Yield strength at max } \\
\text { use temp (ksi) }\end{array}$ & $\begin{array}{c}\begin{array}{c}\text { Stress } \\
(\mathbf{k s i})\end{array} \\
\end{array}$ & $\begin{array}{c}\text { Temp } \\
\left({ }^{\circ} \mathrm{C}\right)\end{array}$ & Environment & Cofficient of thermal expansion & Electrical Resisitivity (ohm-meters) & \\
\hline \multirow{5}{*}{$\begin{array}{l}\text { Rotor/magnetic } \\
\text { bearing }\end{array}$} & Magnetic material & $\begin{array}{l}\text { Fe-Co alloy: possible Hiperco® } \\
\text { Allog } 50 \mathrm{HS} 48.75 \mathrm{wt} \% \mathrm{Co} \text {, bal Fe, } \\
1.90 \mathrm{~V}, 0.30 \mathrm{Nb}, 0.05 \mathrm{Si}, 0.05 \mathrm{Mn}, \\
0.01 \mathrm{C}\end{array}$ & 730 (order-disorder) & & 37 & & & $\begin{array}{l}\text { Hiperco } 0^{\circledast} 50: \text { Mean to } 700^{\circ} \mathrm{C}(\text { ave of } \\
600 \text { and } 800):(10.5+11.3) 10^{-4} / 2=10.9 \\
\times 10^{-4} \text { per }^{\circ} \mathrm{C}\end{array}$ & $\begin{array}{l}\text { Hiperco }^{\circledR} 50 \text { at room temperature: } 400 \\
\text { micro-ohm-mm } \\
\text { ( } 400,000 \text { micro-ohm-m?) }\end{array}$ & \\
\hline & Interleaf insulation & AlSiO BN, MgO, & & & TBD & \multirow{3}{*}{700} & Inert gas? Argon? & & & $\begin{array}{l}\text { Stress due to thermal } \\
\text { expansion of the gas }\end{array}$ \\
\hline & Potting compound & Aremco Ceramacast 584 & & & TBD & & Inert gas? Argon? & & & \multirow[t]{2}{*}{$\begin{array}{l}\text { Stress due to thermal } \\
\text { expansion of the gas }\end{array}$} \\
\hline & Shaft & Inconel $^{\mathbb{Q}} 600$, Haynes $^{\circledR} 214$ & & $\begin{array}{l}\text { Hastelloy }^{\circledR} \mathrm{N}: 28 \text { at } \\
704^{\circ} \mathrm{C} \\
\text { Inconel }^{\circledR} 700: 65 \mathrm{ksi} \\
\text { Haynes }^{\circledR} 214: 77.8 \text { at } \\
760^{\circ} \mathbf{C}\end{array}$ & 92 & & $\begin{array}{l}\text { Internal flow channel } \\
\text { wetted by FLiNaK. } \\
\text { External surface wetted } \\
\text { by inert gas? Argon? }\end{array}$ & 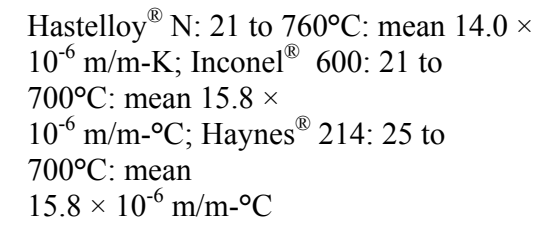 & $\begin{array}{l}\text { Hastelloy }{ }^{\circledR} \mathrm{N} \text { at } 705^{\circ} \mathrm{C}: 1.24 \text { micro- } \\
\text { ohm-m; Inconel }{ }^{\mathbb{\Phi}} 600: 1.13 \text { micro- } \\
\text { ohm-m; Haynes } 214 \text { at } 700^{\circ} \mathrm{C} \text {; } \\
133.7 \text { micro-ohm-cm }\end{array}$ & \\
\hline & Rotor can (seal) & Hastelloy ${ }^{\mathbb{D}} \mathrm{N}$, Nickel 200 & & & 55 & & & $\begin{array}{l}\mathrm{Ni} 200: 21 \text { to } 700^{\circ} \mathrm{C} \text { : mean } 15.8 \times 10-6 \\
\mathrm{~m} / \mathrm{m}-{ }^{\circ} \mathrm{C}\end{array}$ & 0.0430 micro-ohm-cm & $\begin{array}{l}\text { Stress due to thermal } \\
\text { expansion of the gas }\end{array}$ \\
\hline $\begin{array}{l}\text { Inner can (salt } \\
\text { barrier) }\end{array}$ & Material & Hastelloy ${ }^{\circledR}(1 \mathrm{~mm}), \mathrm{SiC}$ & & $\begin{array}{l}\text { Hastelloy }{ }^{\circledR} \mathrm{N}: 28 \text { at } \\
7044^{\circ} \mathrm{C}\end{array}$ & TBD & 700 & $\begin{array}{l}\text { External surface wetted } \\
\text { by FLiNaK. Internal } \\
\text { surface wetted by inert } \\
\text { gas? Argon? }\end{array}$ & $\begin{array}{l}\text { Hastellow }{ }^{\circledR} \mathrm{N}: 21 \text { to } 760^{\circ} \mathrm{C} \text { : mean } 14.0 \times \\
10^{-6} \mathrm{~m} / \mathrm{m}-\mathrm{K}\end{array}$ & $\begin{array}{l}\text { Hastelloy }{ }^{\circledR} \mathrm{N} \text { at } 705^{\circ} \mathrm{C}: 1.24 \text { micro- } \\
\text { ohm-m }\end{array}$ & \\
\hline $\begin{array}{l}\text { Catcher or touch } \\
\text { down bearings }\end{array}$ & & Graphite; ceramic bearing & & & TBD & & & & & $\begin{array}{l}\text { At temperature in the } \\
\text { salt }\end{array}$ \\
\hline \multirow[t]{3}{*}{$\begin{array}{l}\text { Can (outer salt } \\
\text { barrier) }\end{array}$} & Material & Hastelloy $^{\circledR} \mathrm{N}(1 \mathrm{~mm})$ & & & & 700 & $\begin{array}{l}\text { Internal surface wetted } \\
\text { by FLiNaK. External } \\
\text { surface wetted by air? }\end{array}$ & $\begin{array}{l}\text { Hastellow }{ }^{\circledR} \mathrm{N}: 21 \text { to } 760^{\circ} \mathrm{C} \text { : mean } 14.0 \times \\
10^{-6} \mathrm{~m} / \mathrm{m}-\mathrm{K}\end{array}$ & $\begin{array}{l}\text { Hastelloy }{ }^{\circledR} \mathrm{N} \text { at } 705^{\circ} \mathrm{C}: 1.24 \text { micro- } \\
\text { ohm-m }\end{array}$ & \\
\hline & Wire windings & $\begin{array}{l}\mathrm{Ag} \text { or Ag-flashed } \mathrm{Cu} \text { or Ni-flashed } \\
\mathrm{Cu}\end{array}$ & & & TBD & & & Cu: $16.6 \times 10^{-6} \mathrm{~m} / \mathrm{m}-\mathrm{K}$ & $\begin{array}{l}\mathrm{Cu} \text { at } 700^{\circ} \mathrm{C} ; 0.626 \text { micro-ohm-m; } \\
\text { and at } 20^{\circ} \mathrm{C}: 0.0168 \text { micro-ohm-m }\end{array}$ & \\
\hline & Wire insulation & Ceramic coated, S-glass, $\mathrm{Al}_{2} \mathrm{O}_{3}$ & & & TBD & & & & & \\
\hline \multirow[t]{3}{*}{$\begin{array}{l}\text { Stator/magnetic } \\
\text { bearings }\end{array}$} & Magnetic material & $\begin{array}{l}\text { Vacodur }^{\circledR} 50, \text { Hiperco }^{\circledR} 27, \\
\text { Hiperco }^{\circledR} 50 \mathrm{HS}\end{array}$ & & & TBD & & & $\begin{array}{l}\text { Hiperco } 0^{\circledast} 50: \text { Mean to } 700^{\circ} \mathrm{C} \text { (ave of } \\
600 \text { and } 800):(10.5+11.3) 10^{-4} / 2=10.9 \\
\times 10^{-4} \text { per }^{\circ} \mathrm{C}\end{array}$ & $\begin{array}{l}\text { Hiperco }{ }^{\circledR} 50 \text { at room temperature: } 400 \\
\text { micro-ohm-mm } \\
(400,000 \text { micro-ohm-m?) }\end{array}$ & \\
\hline & Structural materials & & & & TBD & & & & & \\
\hline & Thermal insulation & & & & TBD & & & & & $\begin{array}{l}\text { Between } 40 \text { and } \\
750^{\circ} \mathrm{C}\end{array}$ \\
\hline $\begin{array}{l}\text { Structural } \\
\text { support for can, } \\
\text { catcher bearing, } \\
\text { etc }\end{array}$ & & Alloy $800 \mathrm{H}$ & & $\begin{array}{l}\text { Alloy } 800 \text { at } 705^{\circ} \mathrm{C}: \\
25.3 \text { (annealed } 980^{\circ} \mathrm{C} \\
\text { condition); and } 61.4 \\
\text { (cold-drawn rod) }\end{array}$ & TBD & & & $\begin{array}{l}\text { Alloy } 800 \text { at } 700^{\circ} \mathrm{C}: 17.5 \text { micro- } \\
\text { ohy } / \mathrm{m}^{\circ}{ }^{\circ} \mathrm{C}\end{array}$ & $\begin{array}{l}\text { Alloy } 800 \text { at } 700^{\circ} \mathrm{C}: \\
1,251,000 \text { micro-ohm-m }\end{array}$ & $\begin{array}{l}\text { At } 700^{\circ} \mathrm{C} \text {, } \\
\text { interdiffusion effects } \\
\text { over the long term } \\
\text { (under } 20 \text { years; } \\
\text { more likely } 7 \text { years) }\end{array}$ \\
\hline Safety cage & & & & & & & Air outside & & & $\begin{array}{l}\text { High toughness; good } \\
\text { heat transfer }\end{array}$ \\
\hline
\end{tabular}





\section{A.3 RESOLVER MODEL}

Assuming no coupling with other coils, no fringing or leakage, and a series connection of the two excitation coils, the amount of magnetic flux in the excitation coils can be defined by Equation (88).

$$
\varnothing_{\text {exc } \_p 1}=\varnothing_{\text {exc } c p 2}=\frac{2 N_{e x c} i_{e x c}}{2 R_{S_{-} t o o t h}+R_{R_{-} c o r e}+R_{S_{-} c o r e}+R_{g a p_{-} c p 1}(\theta)+R_{g a p_{-} c p 2}(\theta)}
$$

where $N_{e x c}$ is the number of turns in the excitation winding and $i_{e x c}$ is the excitation current. The inductances of the excitation coils are also equal:

$$
L_{\text {exc_c } c 1}=L_{\text {exc_c } c p 2}=\frac{N_{e x c} \varnothing_{e x c_{-} c p 1}}{i_{\text {exc }}}=\frac{2 N_{\text {exc }}{ }^{2}}{2 R_{S_{-} \text {tooth }}+R_{R_{-} \text {core }}+R_{S_{-} c o r e}+R_{\text {gap } \_p 1}(\theta)+R_{\text {gap_cp } 2}(\theta)}
$$

Positive sensor coils are oriented in the same polarity as the excitation coils and therefore the magnetic flux is equal to that of the excitation coils:

$$
\varnothing_{\text {sen_cp1 }}=\varnothing_{\text {sen_ }_{-} c p 2}=\varnothing_{e x c_{-} c p 1}=\varnothing_{\text {exc_cp } 2}
$$

The voltage induced in the series connection of the positive cosine sensor windings is given by

$$
\varepsilon_{\text {sen_cp } 1+2}=-\frac{N_{s e n} d\left(\varnothing_{s e n_{-} c p 1}+\varnothing_{s e n_{-} c p 2}\right)}{d t}=-\frac{N_{s e n} d\left(\varnothing_{e x c_{-} c p 1}+\varnothing_{e x c_{-} c p 2}\right)}{d t} \text {, }
$$

where $N_{s e n}$ is the number of turns in the sensor winding. The same approach can be used to obtain expressions for magnetic flux, inductance, and induced voltage of the other positive cosine excitation and sensor coils:

$$
\begin{aligned}
& \varnothing_{\text {exc_c } c 33}=\varnothing_{\text {exc_cp } 4}=\frac{2 N_{\text {exc }} i_{\text {exc }}}{2 R_{S_{-} t o o t h}+R_{R_{-} c o r e}+R_{S_{-} c o r e}+R_{\text {gap_cp } 3}(\theta)+R_{\text {gap_cp } 4}(\theta)} \\
& L_{e x c_{-} c p 3}=L_{e x c_{-} c p 4}=\frac{N_{e x c} \varnothing_{e x c_{-} c p 3}}{i_{\text {exc }}}=\frac{2 N_{\text {exc }}{ }^{2}}{2 R_{S_{-} t o o t h}+R_{R_{-} c o r e}+R_{S_{-} c o r e}+R_{g a p_{-} c p 3}(\theta)+R_{g a p_{-} c p 4}(\theta)} \\
& \varnothing_{\text {sen_ }_{-} c p 3}=\varnothing_{\text {sen_ }_{-} c p 4}=\varnothing_{\text {exc_ }_{-} c p 3}=\varnothing_{\text {exc_cp } 4} \\
& \varepsilon_{\text {sen_cp3+4 }}=-\frac{N_{s e n} d\left(\varnothing_{s e n_{-} c p 3}+\varnothing_{\text {sen_c } c p 4_{4}}\right)}{d t}=-\frac{N_{s e n} d\left(\varnothing_{\text {exc_cp3 }}+\varnothing_{e x c_{-} c p 4}\right)}{d t}
\end{aligned}
$$

Expressions for magnetic flux and inductance of the negative cosine excitation coils are similar:

$$
\begin{aligned}
& \varnothing_{\text {exc_c } c 1}=\varnothing_{\text {exc_cn2 }}=\frac{2 N_{e x c} i_{e x c}}{2 R_{S_{-} t o o t h}+R_{R_{-} c o r e}+R_{S_{-} c o r e}+R_{\text {gap_c } c 1}(\theta)+R_{g a p_{-} c n 2}(\theta)} \\
& L_{\text {exc_cn }}=L_{\text {exc_c } c n 2}=\frac{N_{\text {exc }} \varnothing_{\text {exc } c n 1}}{i_{\text {exc }}}=\frac{2 N_{\text {exc }}^{2}}{2 R_{S_{-} \text {tooth }}+R_{R_{-} c o r e}+R_{S_{-} c o r e}+R_{\text {gap_cn1 }}(\theta)+R_{\text {gap_cn } 2}(\theta)}
\end{aligned}
$$


However, negative sensor coils have polarities opposite to the excitation coils, and thus magnetic flux in the coils also have opposite polarities:

$$
\varnothing_{\text {sen_cn1 }}=\varnothing_{\text {sen_cn2 }}=-\varnothing_{e x c_{-} c n 1}=-\varnothing_{\text {exc_cn2 }}
$$

Therefore, the induced voltage in the negative cosine sensor winding is :

$$
\varepsilon_{\text {sen_cn1+2 }}=-\frac{N_{s e n} d\left(\varnothing_{\text {sen_cn1 }}+\varnothing_{\text {sen_cn2 }}\right)}{d t}=\frac{N_{s e n} d\left(\varnothing_{e x c_{-} c n 1}+\varnothing_{\text {exc_cn2 }}\right)}{d t}
$$

Similarly, magnetic flux, inductance, and induced voltage of the remaining negative cosine excitation and sensor coils can be defined by

$$
\begin{aligned}
& \varnothing_{\text {exc_cn3 }}=\varnothing_{\text {exc_cn4 }}=\frac{2 N_{\text {exc }} i_{\text {exc }}}{2 R_{S_{-} t o o t h}+R_{R_{-} c o r e}+R_{S_{-} \text {core }}+R_{\text {gap_cn3 }}(\theta)+R_{\text {gap_cn } 4}(\theta)} \\
& L_{\text {exc_cn3 }}=L_{\text {exc_c } c n 4}=\frac{N_{e x c} \varnothing_{e x c_{-} c n 3}}{i_{\text {exc }}}=\frac{2 N_{\text {exc }}{ }^{2}}{2 R_{S_{-} \text {tooth }}+R_{R_{-} \text {core }}+R_{S_{-} c o r e}+R_{\text {gap } \_n 3}(\theta)+R_{\text {gap_cn }}(\theta)} \\
& \varnothing_{\text {sen_cn3 }}=\varnothing_{\text {sen_cn4 }}=-\varnothing_{\text {exc_cn3 }}=-\varnothing_{\text {exc_cn } 4} \\
& \varepsilon_{\text {sen_cn3+4 }}=-\frac{N_{s e n} d\left(\varnothing_{\text {sen_cn3 }}+\varnothing_{\text {sen_cn } 4_{-}}\right)}{d t}=\frac{N_{s e n} d\left(\varnothing_{\text {exc_cn3 }}+\varnothing_{e x c_{-} c n 4}\right)}{d t}
\end{aligned}
$$

With all cosine excitation coils in series, the total corresponding inductance is

$$
L_{e x c_{-} c_{-} t o t a l}=L_{e x c_{-} c p 1}+L_{e x c_{-} c p 2}+L_{e x c_{-} c p 3}+L_{e x c_{-} c p 4}+L_{e x c_{-} c n 1}+L_{e x c_{-} c n 2}+L_{e x c_{-} c n 3}+L_{e x c_{-} c n 4}
$$

And with all cosine sensor coils in series, the total induced voltage is

$$
\varepsilon_{\text {sen_c_total }}=\varepsilon_{\text {sen_cp } 1+2}+\varepsilon_{\text {sen_ }_{-} c p 3+4}+\varepsilon_{\text {sen_cn } 1+2}+\varepsilon_{\text {sen_cn } 3+4}
$$

Deriving expressions for magnetic flux, inductance, and induced voltage for sine excitation and sensing coils in the same manner: 


$$
\begin{aligned}
& \varnothing_{\text {exc_sp } 1}=\varnothing_{\text {exc_sp } 2}=\frac{2 N_{\text {exc }} i_{\text {exc }}}{2 R_{S_{-} \text {tooth }}+R_{R_{-} \text {core }}+R_{S_{-} \text {core }}+R_{\text {gap_sp } 1}(\theta)+R_{\text {gap_sp } 2}(\theta)} \\
& L_{\text {exc } \_s p 1}=L_{\text {exc } \_s p 2}=\frac{N_{e x c} \varnothing_{\text {exc_sp } 1}}{i_{\text {exc }}}=\frac{2 N_{\text {exc }}{ }^{2}}{2 R_{S_{-} \text {tooth }}+R_{R_{-} \text {core }}+R_{S_{-} \text {core }}+R_{\text {gap } \_s p 1}(\theta)+R_{g a p \_s p 2}(\theta)} \\
& \varnothing_{\text {sen_sp1 }}=\varnothing_{\text {sen_sp2 }}=\varnothing_{\text {exc_sp1 }}=\varnothing_{\text {exc_sp2 }} \\
& \varepsilon_{\text {sen_sp1+2 }}=-\frac{N_{s e n} d\left(\varnothing_{\text {sen_sp1 }}+\varnothing_{\text {sen_sp2 }}\right)}{d t}=-\frac{N_{s e n} d\left(\varnothing_{e x c_{\_} s p 1}+\varnothing_{e x c_{\_} s p 2}\right)}{d t} \\
& \varnothing_{\text {exc_sp } 3}=\varnothing_{\text {exc_sp } 4}=\frac{2 N_{\text {exc }} i_{\text {exc }}}{2 R_{S_{-} \text {tooth }}+R_{R_{-} \text {core }}+R_{S_{-} \text {core }}+R_{\text {gap_s } s p 3}(\theta)+R_{\text {gap_sp } 4}(\theta)}
\end{aligned}
$$

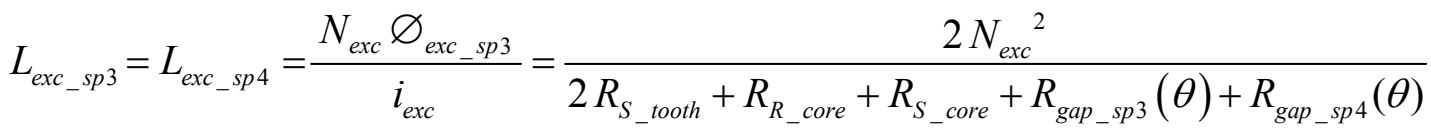

$$
\begin{aligned}
& \varnothing_{\text {sen_sp3 }}=\varnothing_{\text {sen_sp4 }}=\varnothing_{\text {exc_sp3 }}=\varnothing_{\text {exc_sp4 }} \\
& \varepsilon_{\text {sen_sp3+4 }}=-\frac{N_{s e n} d\left(\varnothing_{s e n_{-} s p 3}+\varnothing_{\text {sen_sp4 }}\right)}{d t}=-\frac{N_{s e n} d\left(\varnothing_{e x c_{-} s p 3}+\varnothing_{e x c_{-} s p 4}\right)}{d t} \\
& \varnothing_{\text {exc_sn1 }}=\varnothing_{\text {exc_sn2 }}=\frac{2 N_{\text {exc }} i_{\text {exc }}}{2 R_{S_{-} \text {tooth }}+R_{R_{-} \text {core }}+R_{S_{-} \text {core }}+R_{\text {gap_s sn1 }}(\theta)+R_{\text {gap_s } n 2}(\theta)} \\
& L_{\text {exc_sn1 }}=L_{\text {exc_sn2 }}=\frac{N_{\text {exc }} \varnothing_{\text {exc_sn1 }}}{i_{\text {exc }}}=\frac{2 N_{\text {exc }}{ }^{2}}{2 R_{S_{-} \text {tooth }}+R_{R_{-} \text {core }}+R_{S_{-} \text {core }}+R_{\text {gap_s } s 1}(\theta)+R_{\text {gap_s } s n}(\theta)} \\
& \varnothing_{\text {sen_ssn }}=\varnothing_{\text {sen_sn2 }}=-\varnothing_{\text {exc_sn1 }}=-\varnothing_{\text {exc_sn2 }} \\
& \varepsilon_{\text {sen_sn } \_2}=-\frac{N_{s e n} d\left(\varnothing_{\text {sen_sn1 }}+\varnothing_{\text {sen_sn2 }}\right)}{d t}=\frac{N_{\text {sen }} d\left(\varnothing_{\text {exc_sn1 }}+\varnothing_{\text {exc_sn2 } 2}\right)}{d t} \\
& \varnothing_{\text {exc_sn3 }}=\varnothing_{\text {exc_sn } 4}=\frac{2 N_{\text {exc }} i_{\text {exc }}}{2 R_{S_{-} \text {tooth }}+R_{R_{-} \text {core }}+R_{S_{-} \text {core }}+R_{\text {gap_s } n 3}(\theta)+R_{\text {gap_s } s n 4}(\theta)} \\
& L_{\text {exc } \_s n 3}=L_{\text {exc } \_s n 4}=\frac{N_{\text {exc }} \varnothing_{\text {exc_sn3 }}}{i_{\text {exc }}}=\frac{2 N_{\text {exc }}{ }^{2}}{2 R_{S_{-} \text {tooth }}+R_{R_{-} \text {core }}+R_{S_{-} \text {core }}+R_{\text {gap_s } s n 3}(\theta)+R_{\text {gap_s } n 4}(\theta)} \\
& \varnothing_{\text {sen_sn3 }}=\varnothing_{\text {sen_sn4 }}=-\varnothing_{\text {exc_sn3 }}=-\varnothing_{\text {exc_sn } 4} \\
& \varepsilon_{\text {sen_sn3+4 }}=-\frac{N_{\text {sen }} d\left(\varnothing_{\text {sen_sn3 }}+\varnothing_{\text {sen_sn4 }}\right)}{d t}=\frac{N_{\text {sen }} d\left(\varnothing_{e x c_{-} s n 3}+\varnothing_{\text {exc_sn4 }}\right)}{d t}
\end{aligned}
$$

With all sine excitation coils in series, the total corresponding inductance is

$$
L_{e x c_{-} s_{-} t o t a l}=L_{e x c_{-} s p 1}+L_{e x c_{-} s p 2}+L_{e x c_{-} s p 3}+L_{e x c_{-} s p 4}+L_{e x c_{-} s n 1}+L_{e x c_{-} s n 2}+L_{e x C_{-} s n 3}+L_{e x c_{-} s n 4}
$$

And with all sine sensor coils in series, the total induced voltage is

$$
\varepsilon_{s e n_{-} s_{-} t o t a l}=\varepsilon_{\text {sen_- } c p 1+2}+\varepsilon_{\text {sen_ } c p 3+4}+\varepsilon_{\text {sen_cnl+2 }}+\varepsilon_{\text {sen_cn3 } c 4}
$$


Sine and cosine excitation coils can be connected in series and the total corresponding inductance is

$$
L_{\text {exc_total }}=L_{\text {exc_c_total }}+L_{\text {exc_s_total }}
$$

\section{A.4 WIRE AND COIL MATERIAL SELECTION}

Wire. There are two classes of conductors: (1) low resistance conductors used as magnet wire and (2) a broad class of special conductors designed for specific requirements. For the stator windings, the conductor will need to be low resistance magnet wire designed for high-temperature use, with which an oxidation resistant cladding will be used. Examples of the second conductor class are pure metals such as nickel or platinum for use as resistance thermometer wires, which have high resistance to oxidation. Likewise, high resistance wires, normally alloys of nickel and chromium, are oxidation resistant. (e.g., Stablohm ${ }^{\circledR} 675$ and 800).

Copper, the electrical conductor of choice for most coil winding applications, has a maximum operating temperature of $315^{\circ} \mathrm{C}$ in air and conducts electricity at 97 percent that of silver. Pure copper undergoes severe oxidation in the temperature range of $350^{\circ} \mathrm{C}$ to $1050^{\circ} \mathrm{C}$, due to outward migration of copper atoms to form $\mathrm{Cu}_{2} \mathrm{O}$ at the scale gas interface. ${ }^{59}$

For most high-temperature applications of copper wire, a thin nickel coating is employed to minimize copper oxidation, improve mechanical properties, and limit copper diffusion into adjacent materials at elevated temperatures. Typical nickel layer thickness over copper wire is $10 \mu \mathrm{m}$ or greater. Other coatings are also feasible and may be desired such as silver. Shown in Table A-10 are data related to evaporation of several metals at various partial pressures and temperatures. Note that the vapor pressure of silver (not on table) is virtually the same as manganese. At temperatures around $700^{\circ} \mathrm{C}$, nickel and silver remain solid, have low vapor pressure, and low evaporation rates. The right side of the table, based on the Langmuir evaporation Equation (103), illustrates that as partial pressure increases, the number of atoms leaving the surface increases as well.

$$
\frac{d N}{d t}=\frac{p}{\sqrt{2 \pi m k T}},
$$

where $N$ is the density of atoms, $p$ is the partial pressure, $m$ is the atomic mass, $k$ is Boltzmann constant, and $T$ is absolute temperature. 
Table A-10. Metal evaporation sensitivity to temperature and pressure for potential wire material selection

\begin{tabular}{|c|c|c|c|c|c|c|c|c|c|c|c|c|c|c|c|}
\hline \multirow{2}{*}{$\begin{array}{l}\text { Element } \\
\text { (Symbol) }\end{array}$} & \multirow{2}{*}{$\begin{array}{l}\text { Element } \\
\text { (Name) }\end{array}$} & \multirow{2}{*}{\begin{tabular}{|c|}
$\begin{array}{c}\text { Molecular } \\
\text { Mass }\end{array}$ \\
(amu) \\
\end{tabular}} & \multirow{2}{*}{$\begin{array}{c}\text { Melting } \\
\text { Point } \\
\left({ }^{\circ} \mathrm{C}\right) \\
\end{array}$} & \multicolumn{6}{|c|}{ Temperature (C) for Partial Pressure (Atmospheres) } & \multicolumn{6}{|c|}{$\begin{array}{l}\text { Log Flux of Metal Atoms at Temperature and Partial Pressures } \\
\text { (Relative to Platinum at } 1492^{\circ} \mathrm{C} \text { ) }\end{array}$} \\
\hline & & & & 1.31E-08 & 1.31E-07 & $1.31 E-06$ & 1.31E-05 & 1.31E-04 & $1.31 E-03$ & $1.31 E-08$ & $1.31 E-07$ & $1.31 E-06$ & $1.31 E-05$ & $1.31 E-04$ & $1.31 E-03$ \\
\hline w & Tungsten & 184 & 3400 & 2407 & 2567 & 2757 & 2977 & 3227 & 3537 & 0.01 & 1.01 & 2.01 & 3.01 & 4.01 & 5.01 \\
\hline $\mathrm{Re}$ & Rhenium & 186 & 3182 & 2217 & 2387 & 2587 & 2807 & 3067 & 3407 & 0.01 & 1.01 & 2.01 & 3.01 & 4.01 & 5.01 \\
\hline $\mathrm{Nb}$ & Niobium & 93 & 2470 & 1987 & 2127 & 2277 & 2447 & 2657 & 2897 & 0.16 & 1.16 & 2.16 & 3.16 & 4.16 & 5.16 \\
\hline Mo & Molybdenum & 96 & 2620 & 1822 & 1957 & 2117 & 2307 & 2527 & 2787 & 0.15 & 1.15 & 2.15 & 3.15 & 4.15 & 5.15 \\
\hline $\mathrm{Pt}$ & Platinum & 195 & 1770 & 1492 & 1612 & 1747 & 1907 & 2097 & 2317 & 0.00 & 1.00 & 2.00 & 3.00 & 4.00 & 5.00 \\
\hline $\mathrm{Rh}$ & Rhodium & 103 & 1965 & 1472 & 1582 & 1707 & 1857 & 2037 & 2247 & 0.14 & 1.14 & 2.14 & 3.14 & 4.14 & 5.14 \\
\hline $\mathrm{Si}$ & Silicon & 28 & 1411 & 1147 & 1237 & 1337 & 1472 & 1632 & 1817 & 0.42 & 1.42 & 2.42 & 3.42 & 4.42 & 5.42 \\
\hline $\mathrm{Ni}$ & Nickel & 59 & 1453 & 1072 & 1157 & 1262 & 1382 & 1527 & 1697 & 0.26 & 1.26 & 2.26 & 3.26 & 4.26 & 5.26 \\
\hline $\mathrm{Pd}$ & Palladium & 106 & 1555 & 992 & 1082 & 1192 & 1317 & 1462 & 1647 & 0.13 & 1.13 & 2.13 & 3.13 & 4.13 & 5.13 \\
\hline $\mathrm{Fe}$ & Iron & 56 & 1536 & 1032 & 1127 & 1227 & 1342 & 1477 & 1647 & 0.27 & 1.27 & 2.27 & 3.27 & 4.27 & 5.27 \\
\hline $\mathrm{Au}$ & Gold & 197 & 1063 & 947 & 1032 & 1132 & 1252 & 1397 & 1567 & 0.00 & 1.00 & 2.00 & 3.00 & 4.00 & 5.00 \\
\hline $\mathrm{Cr}$ & Chromium & 52 & 1860 & 977 & 1062 & 1157 & 1267 & 1397 & 1552 & 0.29 & 1.29 & 2.29 & 3.29 & 4.29 & 5.29 \\
\hline $\mathrm{Cu}$ & Copper & 64 & 1084 & 852 & 937 & 1027 & 1132 & 1257 & 1417 & 0.24 & 1.24 & 2.24 & 3.24 & 4.24 & 5.24 \\
\hline $\mathrm{Al}$ & Aluminum & 27 & 660 & 812 & 887 & 972 & 1082 & 1217 & 1367 & 0.43 & 1.43 & 2.43 & 3.43 & 4.43 & 5.43 \\
\hline In & Indium & 115 & 156.6 & 597 & 664 & 742 & 837 & 947 & 1082 & 0.11 & 1.11 & 2.11 & 3.11 & 4.11 & 5.11 \\
\hline $\mathrm{Mn}$ & Manganese & 55 & 1244 & 611 & 675 & 747 & 837 & 937 & 1082 & 0.27 & 1.27 & 2.27 & 3.27 & 4.27 & 5.27 \\
\hline $\mathrm{Mg}$ & Magnesium & 24 & 650 & 246 & 282 & 327 & 377 & 439 & 509 & 0.45 & 1.45 & 2.45 & 3.45 & 4.45 & 5.45 \\
\hline
\end{tabular}

Wire insulation. Organic and polymer insulations are not usable for applications above $250^{\circ} \mathrm{C}$. For $700^{\circ} \mathrm{C}$ operating temperatures, a ceramic material is the only viable option. The ceramic must be applied to the wire in green (uncured) state to allow spindling the wire on a bobbin - a hardened or glassy material would continually crack during winding. Note that some cracking is expected, which is the reason for potting the final wound bobbin (discussed below under potting).

An example ceramic insulation for nickel coated copper wire is Cercal and Alcal "E." Both insulation types are manufactured by California Fine Wire Company, Grover Beach, California. Cercal has a composition of oxides of lead, silicon, bismuth, and zinc. Alcal "E" has a composition of aluminum oxide and silicon dioxide. The maximum operating temperature of Cercal is just above $700^{\circ} \mathrm{C}$ whereas Alcal "E" functions to $880^{\circ} \mathrm{C}$.

The primary difference between the Cercal and the Alcal Ceramic is that the Cercal requires a conductor that can be oxidized on its surface in order to provide for bonding between the conductor and the ceramic. Table A-11 compares properties of the two insulation products. Based on data from the manufacturer, applicable conductors are as follows:

- Nickel clad copper,

- Nickel clad sliver,

- Aluminum and aluminum alloys,

- Aluminum clad copper,

- Constantan,

- Chromel P, and

- Alumel.

Alcal type "E" ceramic insulation can be applied to the above materials and the following as well:

- Platinum,

- Gold,

- Silver,

- Palladium,

- Rhenium and most precious metal alloys, 
- Stainless steels, and

- Nickel-chromium alloys.

Table A-11. Comparison of ceramic insulation available from California Fine Wire Company

\begin{tabular}{|c|c|c|}
\hline Property & Cercal & Alcal \\
\hline Mechanical & $\begin{array}{l}\text { Semi-cured material is flexible. Wire is not } \\
\text { flexible after final cure }\end{array}$ & $\begin{array}{l}\text { Semi-cured material is flexible. Wire is not } \\
\text { flexible after final cure }\end{array}$ \\
\hline Insulation & Refractory glass metal compound & $\begin{array}{l}\text { Aluminum Silicon compound consisting of } \\
\text { Alumina and Silicon dioxide }\end{array}$ \\
\hline Temperature Range & $\begin{array}{l}186^{\circ} \mathrm{C} \text { to } 1282^{\circ} \mathrm{C} \text {. Normal operating } \\
\text { temperature is } 600^{\circ} \mathrm{C}\end{array}$ & $\begin{array}{l}38^{\circ} \mathrm{C} \text { to } 871^{\circ} \mathrm{C} \text {. Normal operating } \\
\text { temperature is } 700^{\circ} \mathrm{C}\end{array}$ \\
\hline Thermal Shock & $\begin{array}{l}980^{\circ} \mathrm{C} \text { to ambient in two seconds with no } \\
\text { detectable deterioration }\end{array}$ & $\begin{array}{l}980^{\circ} \mathrm{C} \text { to ambient in two seconds with no } \\
\text { detectable deterioration }\end{array}$ \\
\hline \multicolumn{3}{|l|}{ Service Life } \\
\hline$+420^{\circ} \mathrm{C}$ & 5000 hours & exceeds 5000 hours \\
\hline$+530^{\circ} \mathrm{C}$ & 2500 hours & exceeds 2500 hours \\
\hline$+620^{\circ} \mathrm{C}$ & 1000 hours & exceeds 1000 hours \\
\hline$+700^{\circ} \mathrm{C}$ & 500 hours & exceeds 1000 hours \\
\hline$+870^{\circ} \mathrm{C}$ & N/A & exceeds 1000 hours \\
\hline Voltage Rating & $\begin{array}{l}600 \text { volts D.C. nominal. Voltage and } \\
\text { leakage ratings vary with insulation } \\
\text { thickness. }\end{array}$ & $\begin{array}{l}600 \text { volts D.C. nominal. Voltage and } \\
\text { leakage ratings vary with insulation } \\
\text { thickness. }\end{array}$ \\
\hline
\end{tabular}

High temperature wire of similar characteristics is available from other manufacturers such as Karl Schupp. Nickel clad copper wire with ceramic insulation is a special order component.

\section{Coil bobbin}

The power and sensor coils cannot be wound directly on the ferromagnetic core structures because of mechanical difficulty in accomplishing a high-quality wind and because both an electrical and thermal insulator is needed between the coil and metallic structure. For this reason, preformed coil bobbins will be needed. The bobbin material should be compatible in thermal expansion with the metal (see Table A-12) and not become a significant conductor at higher temperatures because of increased ion mobility (see Fig. A-7 for an example). The bobbin may also have to accommodate addition insulation layers between winding layers. Experience from high-temperature transformer operation indicates that layer-to-layer voltage arc-over is a valid failure mode. 
Table A-12. Characteristics of insulating materials for preformed coil bobbin stock

\begin{tabular}{lcccc}
\hline \multicolumn{1}{c}{ Insulator } & $\begin{array}{c}\text { Minimum } \\
\text { Purity (\%) }\end{array}$ & $\begin{array}{c}\text { Melting } \\
\text { Point (K) }\end{array}$ & $\begin{array}{c}\text { Usable } \\
\text { Temperature }\left({ }^{\circ} \mathbf{C}\right)\end{array}$ & $\begin{array}{c}\text { Thermal Expansion } \\
\left(\times \mathbf{1 0}^{-6} /{ }^{\circ} \mathbf{C}\right)\end{array}$ \\
\hline Magnesia $(\mathrm{MgO})$ & 99.4 & 3073 & 1650 & 11.3 \\
Alumina $\left(\mathrm{Al}_{2} \mathrm{O}_{3}\right)$ & 99.5 & 2303 & 1650 & 9.2 \\
Zirconia $\left(\mathrm{ZrO}_{2}\right)^{*}$ & 99.4 & 2693 & 650 & 10.5 \\
Beryllia $(\mathrm{BeO})$ & 99.8 & 2843 & 2315 & 9.2 \\
Thoria $\left(\mathrm{ThO}_{2}\right)$ & 99.5 & 3323 & 2500 & 9.1 \\
Hafnia $\left(\mathrm{HfO}_{2}\right)$ & 99.8 & 3173 & 2000 & 7.9 \\
Yttria $\left(\mathrm{Y}_{2} \mathrm{O}_{3}\right)$ & 99.8 & 2683 & 2000 & 8.1 \\
Silicon $\mathrm{Carbide}(\mathrm{SiC})_{\text {Silicon Nitride }\left(\mathrm{Si}_{3} \mathrm{~N}_{4}\right)}$ & & & & 4.4 \\
\hline
\end{tabular}

* Pure zirconia is rarely used but rather stabilized ceramic mixtures.

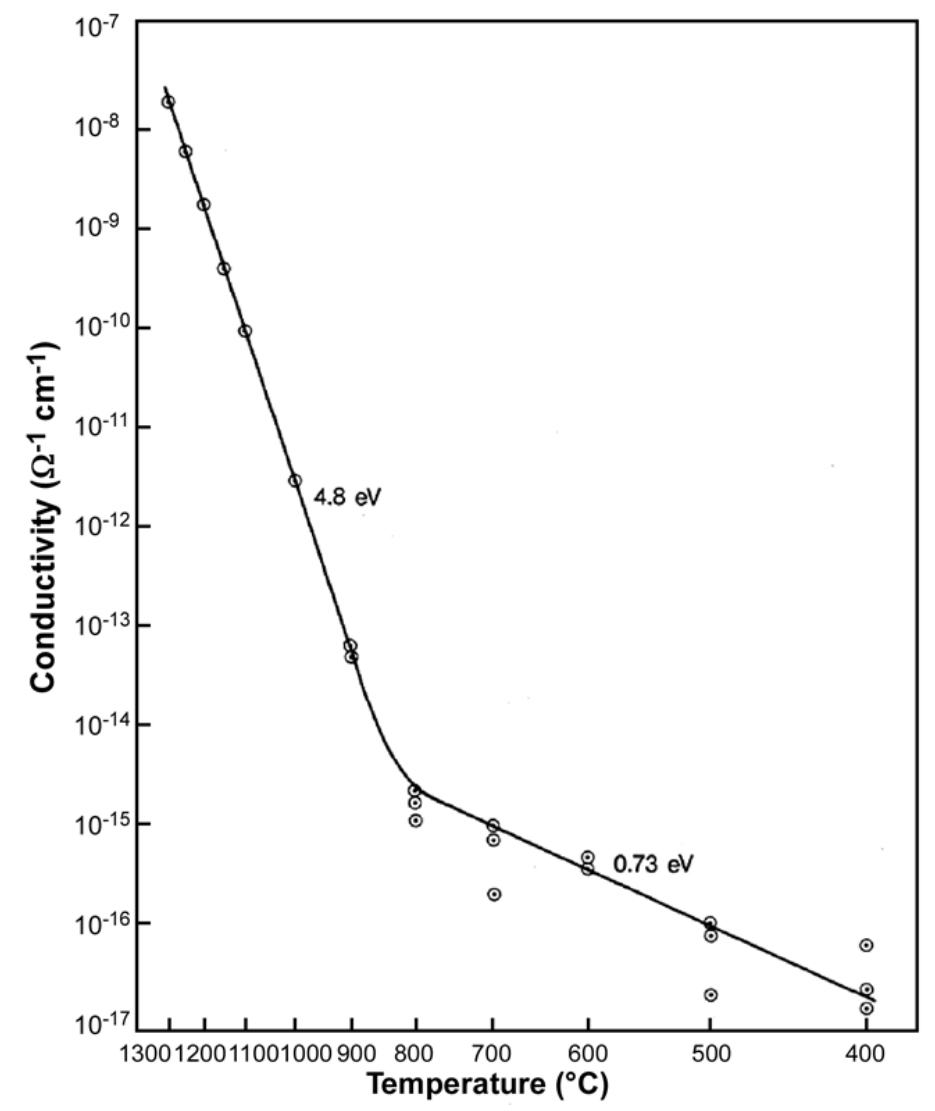

Fig. A-7. Conductivity versus temperature for high purity $\mathrm{Al}_{2} \mathrm{O}_{3}$ (Adapted from Will, deLorenzi, and Janora ${ }^{60}$ ). 


\section{Potting}

For long-term reliable use, the ceramic insulated wire must be potted to (1) block moisture penetration, (2) stabilize wire movement, and (3) fill cracks and gaps in the cured winding insulation. It is important that the potting compound be compatible with the ceramic insulated wire. Several potting compounds are available from California fine wire as well as others for such a purpose. Several processing steps may be required in which a base cement is applied then a subsequent top coat of moisture resistant compound. As an example, the top-coat material designated type 7 by California Fine Wire has good electrical resistance to $700^{\circ} \mathrm{C}$ and excellent moisture resistance. It cures at $845^{\circ} \mathrm{C}$.

\section{Wire size}

Wire size and organization for the solenoid coils of the main drive and suspension bearings is selected according to skin depth, current carrying capacity, and ease of winding. It is common to run many small diameter parallel wires rather than use a single larger conductor because the skin depth causes an increase in effective resistance with increasing frequency. The skin depth $\delta$ (in $\mu \mathrm{m}$ ) is approximated by Equation (104).

$$
\delta=\sqrt{\frac{2 \rho}{\omega \mu}},
$$

where

$$
\begin{aligned}
& \rho=\text { resistivity of the conductor } \\
& \omega=\text { angular frequency of current }=2 \pi \times \text { frequency } \\
& \mu=\text { absolute magnetic permeability of the conductor. }
\end{aligned}
$$

The skin depths for several metals over a frequency range of $10 \mathrm{~Hz}$ to $1 \mathrm{MHz}$ is graphically represented in Fig. A-8.

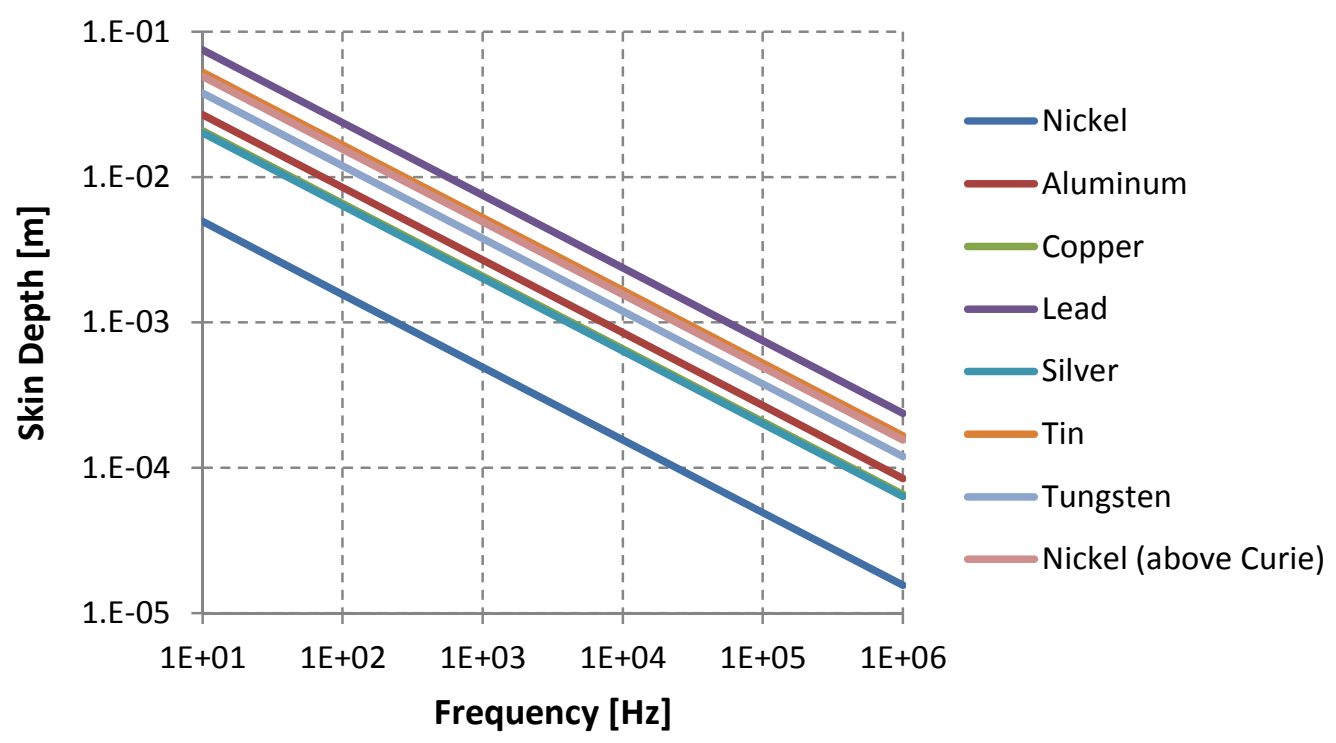

Fig. A-8. Skin depths for several metals. 
To achieve the combined conductivity of parallel conductors at frequency, it is necessary to insulate each wire strand individually, which is a form of Litz wire. ${ }^{\dagger}$ Electrical resistance, linear inductance, and maximum frequency (based on uniformly carrying current across wire diameter) are shown in

Table A-13. As an extreme example, for winding a bearing stator coil to carry current up to $150 \mathrm{kHz}$, one would select 28 AWG wire size from the table. However, to achieve a total resistance of $1 \Omega / \mathrm{km}$ equivalent for the parallel combination would require 213 individual strands. These values are for example only. For practical engineering, it may be necessary to accept a slightly less than full skin depth; further, the wire supplier may only have a limited selection of sizes available for high temperature applications. The allowable total resistance value of a coil is arrived at by determining the maximum heat generation following $P=I^{2} R$, which must be carried away by the thermal system.

Copper wire should be clad with protective metal for reducing copper oxidation and diffusion to enable long-term survival of the stator windings at high-temperatures (see Fig. A-9). From Fig. A-8, the shallower skin depth of nickel below the Curie temperature $\left(358^{\circ} \mathrm{C}\right)$ increases the wires effective resistance. At high frequency, the currents are forced to travel through a shallower annular region thereby increasing the resistance. Nickel above the Curie temperature (see Fig. 72 has a greatly increased depth of penetration and therefore lower resistance. Because the fluoride salt melts above the Curie temperature, nickel will make a reasonable clad material. The skin depth at $10 \mathrm{kHz}$ is large enough that a $1 \mu \mathrm{m}$ cladding will not appreciably increase the effective resistance. Note that silver can also be an alternative cladding material although NASA had experienced some silver migration as discussed in Section 2.3.

Table A-13. Copper wire parameters by size

\begin{tabular}{ccccccccc}
\hline AWG & $\begin{array}{c}\text { Diameter } \\
{[\mathbf{i n} .]}\end{array}$ & $\begin{array}{c}\text { Diameter } \\
{[\mathbf{m m}]}\end{array}$ & $\begin{array}{c}\text { Area } \\
{\left[\mathbf{m m}^{2}\right]}\end{array}$ & $\begin{array}{c}\text { Resistance } \\
{[\mathbf{\Omega} / \mathbf{1 0 0 0}} \\
\mathbf{f t}]\end{array}$ & $\begin{array}{c}\text { Resistance } \\
{[\mathbf{\Omega} / \mathbf{k m}]}\end{array}$ & $\begin{array}{c}\text { Max } \\
\text { Current } \\
{[\text { Amperes }]}\end{array}$ & $\begin{array}{c}\text { Inductance } \\
{[\mathbf{n H} / \mathbf{m}]}\end{array}$ & $\begin{array}{c}\text { Max Frequency } \\
\mathbf{1 0 0 \% \text { skin }} \\
\text { depth }\end{array}$ \\
\hline 0000 & 0.46 & 11.684 & 107 & 0.049 & 0.16072 & 302 & 568 & $125 \mathrm{~Hz}$ \\
000 & 0.4096 & 10.40384 & 85 & 0.0618 & 0.202704 & 239 & 590 & $160 \mathrm{~Hz}$ \\
00 & 0.3648 & 9.26592 & 67.4 & 0.0779 & 0.255512 & 190 & 612 & $200 \mathrm{~Hz}$ \\
0 & 0.3249 & 8.25246 & 53.5 & 0.0983 & 0.322424 & 150 & 635 & $250 \mathrm{~Hz}$ \\
1 & 0.2893 & 7.34822 & 42.4 & 0.1239 & 0.406392 & 119 & 657 & $325 \mathrm{~Hz}$ \\
2 & 0.2576 & 6.54304 & 33.6 & 0.1563 & 0.512664 & 94 & 679 & $410 \mathrm{~Hz}$ \\
3 & 0.2294 & 5.82676 & 26.7 & 0.197 & 0.64616 & 75 & 702 & $500 \mathrm{~Hz}$ \\
4 & 0.2043 & 5.18922 & 21.2 & 0.2485 & 0.81508 & 60 & 724 & $650 \mathrm{~Hz}$ \\
5 & 0.1819 & 4.62026 & 16.8 & 0.3133 & 1.027624 & 47 & 747 & $810 \mathrm{~Hz}$ \\
6 & 0.162 & 4.1148 & 13.3 & 0.3951 & 1.295928 & 37 & 770 & $1100 \mathrm{~Hz}$ \\
7 & 0.1443 & 3.66522 & 10.5 & 0.4982 & 1.634096 & 30 & 792 & $1300 \mathrm{~Hz}$ \\
8 & 0.1285 & 3.2639 & 8.37 & 0.6282 & 2.060496 & 24 & 815 & $1650 \mathrm{~Hz}$ \\
9 & 0.1144 & 2.90576 & 6.63 & 0.7921 & 2.598088 & 19 & 838 & $2050 \mathrm{~Hz}$ \\
\hline
\end{tabular}

\footnotetext{
${ }^{\dagger}$ Litz wire is a type of cable used carry alternating current. The wire is designed to reduce skin effect and proximity effect losses in conductors at high frequencies. The cable consists of many smaller strands, individually insulated and twisted together, following carefully prescribed patterns to balance the proportion that each strand is exposed to the outside of the conductor.
} 
Table A-13. Copper wire parameters by size (continued)

\begin{tabular}{|c|c|c|c|c|c|c|c|c|}
\hline AWG & $\begin{array}{c}\text { Diameter } \\
\text { [in.] }\end{array}$ & $\begin{array}{c}\text { Diameter } \\
{[\mathrm{mm}]}\end{array}$ & $\begin{array}{c}\text { Area } \\
{\left[\mathrm{mm}^{2}\right]}\end{array}$ & $\begin{array}{c}\text { Resistance } \\
{[\Omega / 1000} \\
\mathbf{f t}]\end{array}$ & $\begin{array}{c}\text { Resistance } \\
{[\Omega / \mathbf{k m}]}\end{array}$ & $\begin{array}{c}\text { Max } \\
\text { Current } \\
\text { [Amperes] }\end{array}$ & $\begin{array}{c}\text { Inductance } \\
{[\mathrm{nH} / \mathrm{m}]}\end{array}$ & $\begin{array}{c}\text { Max Frequency } \\
\begin{array}{c}100 \% \text { skin } \\
\text { depth }\end{array}\end{array}$ \\
\hline 10 & 0.1019 & 2.58826 & 5.26 & 0.9989 & 3.276392 & 15 & 861 & $2600 \mathrm{~Hz}$ \\
\hline 11 & 0.0907 & 2.30378 & 4.17 & 1.26 & 4.1328 & 12 & 884 & $3200 \mathrm{~Hz}$ \\
\hline 12 & 0.0808 & 2.05232 & 3.31 & 1.588 & 5.20864 & 9.3 & 907 & $4150 \mathrm{~Hz}$ \\
\hline 13 & 0.072 & 1.8288 & 2.62 & 2.003 & 6.56984 & 7.4 & 930 & $5300 \mathrm{~Hz}$ \\
\hline 14 & 0.0641 & 1.62814 & 2.08 & 2.525 & 8.282 & 5.9 & 953 & $6700 \mathrm{~Hz}$ \\
\hline 15 & 0.0571 & 1.45034 & 1.65 & 3.184 & 10.44352 & 4.7 & 976 & $8250 \mathrm{~Hz}$ \\
\hline 16 & 0.0508 & 1.29032 & 1.31 & 4.016 & 13.17248 & 3.7 & 999 & $11 \mathrm{k} \mathrm{Hz}$ \\
\hline 17 & 0.0453 & 1.15062 & 1.04 & 5.064 & 16.60992 & 2.9 & 1022 & $13 \mathrm{k} \mathrm{Hz}$ \\
\hline 18 & 0.0403 & 1.02362 & 0.823 & 6.385 & 20.9428 & 2.3 & 1045 & $17 \mathrm{kHz}$ \\
\hline 19 & 0.0359 & 0.91186 & 0.653 & 8.051 & 26.40728 & 1.8 & 1068 & $21 \mathrm{kHz}$ \\
\hline 20 & 0.032 & 0.8128 & 0.518 & 10.15 & 33.292 & 1.5 & 1091 & $27 \mathrm{kHz}$ \\
\hline 21 & 0.0285 & 0.7239 & 0.41 & 12.8 & 41.984 & 1.2 & 1114 & $33 \mathrm{kHz}$ \\
\hline 22 & 0.0254 & 0.64516 & 0.326 & 16.14 & 52.9392 & 0.92 & 1137 & $42 \mathrm{kHz}$ \\
\hline 23 & 0.0226 & 0.57404 & 0.258 & 20.36 & 66.7808 & 0.729 & 1160 & $53 \mathrm{kHz}$ \\
\hline 24 & 0.0201 & 0.51054 & 0.205 & 25.67 & 84.1976 & 0.577 & 1184 & $68 \mathrm{kHz}$ \\
\hline 25 & 0.0179 & 0.45466 & 0.162 & 32.37 & 106.1736 & 0.457 & 1207 & $85 \mathrm{kHz}$ \\
\hline 26 & 0.0159 & 0.40386 & 0.129 & 40.81 & 133.8568 & 0.361 & 1230 & $107 \mathrm{kHz}$ \\
\hline 27 & 0.0142 & 0.36068 & 0.102 & 51.47 & 168.8216 & 0.288 & 1253 & $130 \mathrm{kHz}$ \\
\hline 28 & 0.0126 & 0.32004 & 0.081 & 64.9 & 212.872 & 0.226 & 1277 & $170 \mathrm{kHz}$ \\
\hline 29 & 0.0113 & 0.28702 & 0.0642 & 81.83 & 268.4024 & 0.182 & 1298 & $210 \mathrm{kHz}$ \\
\hline 30 & 0.01 & 0.254 & 0.0509 & 103.2 & 338.496 & 0.142 & 1323 & $270 \mathrm{kHz}$ \\
\hline 31 & 0.0089 & 0.22606 & 0.0404 & 130.1 & 426.728 & 0.113 & 1346 & $340 \mathrm{kHz}$ \\
\hline 32 & 0.008 & 0.2032 & 0.032 & 164.1 & 538.248 & 0.091 & 1367 & $430 \mathrm{kHz}$ \\
\hline 33 & 0.0071 & 0.18034 & 0.0254 & 206.9 & 678.632 & 0.072 & 1391 & $540 \mathrm{kHz}$ \\
\hline 34 & 0.0063 & 0.16002 & 0.0201 & 260.9 & 855.752 & 0.056 & 1415 & $690 \mathrm{kHz}$ \\
\hline 35 & 0.0056 & 0.14224 & 0.016 & 329 & 1079.12 & 0.044 & 1439 & $870 \mathrm{kHz}$ \\
\hline 36 & 0.005 & 0.127 & 0.0127 & 414.8 & 1360 & 0.035 & 1461 & $1100 \mathrm{kHz}$ \\
\hline 37 & 0.0045 & 0.1143 & 0.01 & 523.1 & 1715 & 0.0289 & 1482 & $1350 \mathrm{kHz}$ \\
\hline 38 & 0.004 & 0.1016 & 0.00797 & 659.6 & 2163 & 0.0228 & 1506 & $1750 \mathrm{kHz}$ \\
\hline 39 & 0.0035 & 0.0889 & 0.00632 & 831.8 & 2728 & 0.0175 & 1533 & $2250 \mathrm{kHz}$ \\
\hline 40 & 0.0031 & 0.07874 & 0.00501 & 1049 & 3440 & 0.0137 & 1557 & $2900 \mathrm{kHz}$ \\
\hline
\end{tabular}




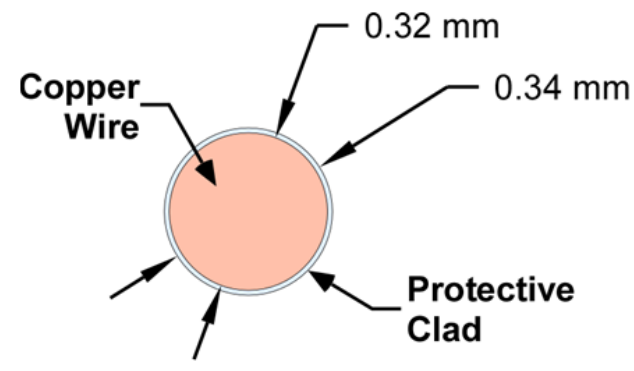

Fig. A-9. Clad wire cross section shown for $28 \mathrm{AWG} \mathrm{Cu}$ with $10 \mu \mathrm{m} \mathrm{Ni}$ Cladding.

\section{Terminations}

An operating temperature of nominally $700^{\circ} \mathrm{C}$ with transients to $750^{\circ} \mathrm{C}$ prohibits and traditional soldering of wiring terminations. A solid galvanic bond between metals is required because of the wide temperature range - room temperature to $700^{\circ} \mathrm{C}$. This requirement prohibits the use of connectors. The only feasible electrical wiring joining methods are welding or brazing. Nickel braze is common as is silver. The braze operation should leave the cladding material covering all copper part.

\section{A.5 FAILURE MODES AND EFFECTS ANALYSIS}

Two partially independent FMEA studies were conducted on the preliminary design of the canned rotor, reluctance motor. The first analysis looked at the failure modes of the magnetic suspension bearing systems and of the main motor drive systems. The second analysis grouped the various motor systems together and categorized components by mechanical, electronic, or control designation. All of these studies are reproduced in this appendix. The study results have directly contributed to the strategies and decisions made in developing a conceptual design of the embedded sensors and controls as well as the motor mechanical and magnetic design.

The design parameters for the mechanical, electronic, and control systems are detailed in Tables A-14, A-15, A-16, A-17, and A-18. 

Table A-14. Mechanical system

\begin{tabular}{|c|c|c|c|c|c|c|c|c|}
\hline & Component & Function & Potential Failure Modes & Potential Cause of Failure & $\begin{array}{l}\text { Potential Effect of Failure } \\
\text { Mode }\end{array}$ & Control Requirement & Detection Method & Corrective Action \\
\hline \multirow{3}{*}{ 燕 } & \multirow[t]{3}{*}{$\overline{\text { Axle (Shaft) }}$} & \multirow[t]{3}{*}{$\begin{array}{l}\text { Rotate about itself; Contain the } \\
\text { flow of salt }\end{array}$} & 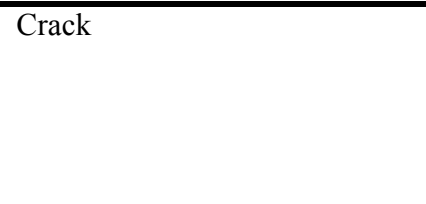 & $\begin{array}{l}\text { Thermal Stresses; Shearing; } \\
\text { Excessive Pressure; Latent Flaw; } \\
\text { Corrosion from salt (coating } \\
\text { fails); Excessive Vibrations }\end{array}$ & $\begin{array}{l}\text { salt leakage(if salt is contained } \\
\text { inside axle); corrosion of rotor } \\
\text { laminations motor failure; slowed } \\
\text { movements of impeller; loss of } \\
\text { coolant flow; }\end{array}$ & $\begin{array}{l}\text { rapid stop; speed reduction of } \\
\text { motor to complete stop; catcher } \\
\text { bearings }\end{array}$ & $\begin{array}{l}\text { temperature sensor; Vibration } \\
\text { sensors; positional sensors; }\end{array}$ & $\begin{array}{l}\text { system would have to be replaced } \\
\text { and repaired (extra motor to be } \\
\text { switched in) }\end{array}$ \\
\hline & & & Detach from Rotor & $\begin{array}{l}\text { Thermal Stresses; Shearing; } \\
\text { Break in Rotor Laminations; } \\
\text { Latent Flaw; Excessive } \\
\text { Vibrations; }\end{array}$ & $\begin{array}{l}\text { loss of rotation; impeller slowed; } \\
\text { loss of coolant flow; rotor crack; } \\
\text { corrosion of rotor; motor failure; }\end{array}$ & $\begin{array}{l}\text { rapid stop; speed reduction of } \\
\text { motor to complete stop; catcher } \\
\text { bearings }\end{array}$ & $\begin{array}{l}\text { temperature sensor; Vibration } \\
\text { sensors; positional sensors; }\end{array}$ & $\begin{array}{l}\text { system would have to be replaced } \\
\text { and repaired (extra motor to be } \\
\text { switched in) }\end{array}$ \\
\hline & & & Detach from Impeller & $\begin{array}{l}\text { Thermal Stresses; Shearing; } \\
\text { Latent Flaw; Excessive } \\
\text { Vibrations; }\end{array}$ & $\begin{array}{l}\text { rotation of impeller slowed; } \\
\text { coolant flow stopped; motor } \\
\text { failure; out of control rotor (the } \\
\text { loss of weight from impeller will } \\
\text { result in the system trying to } \\
\text { compensate for that) }\end{array}$ & $\begin{array}{l}\text { rapid stop; speed reduction of } \\
\text { motor to complete stop; catcher } \\
\text { bearings }\end{array}$ & $\begin{array}{l}\text { temperature sensor; Vibration } \\
\text { sensors; positional sensors; flow } \\
\text { meter }\end{array}$ & $\begin{array}{l}\text { system would have to be replaced } \\
\text { and repaired (extra motor to be } \\
\text { switched in) }\end{array}$ \\
\hline \multirow{5}{*}{ 蒿 } & \multirow[t]{5}{*}{$\begin{array}{l}\text { Rotor Laminations } \\
\text { (Inner Magnetic Bearing } \\
\text { Laminations) }\end{array}$} & \multirow{5}{*}{$\begin{array}{l}\text { Support Structure of magnetic } \\
\text { bearing laminations and provide } \\
\text { rotational torque to the axle (to } \\
\text { drive motor) with potential } \\
\text { fiduciary mark for location } \\
\text { requirements }\end{array}$} & Break Apart & $\begin{array}{l}\text { Thermal Stresses; Faulty } \\
\text { Welding; Latent Flaw; Shearing; } \\
\text { Crack in a single Lamination }\end{array}$ & $\begin{array}{l}\text { incorrect positioning sensors; loss } \\
\text { of rotational speed; }\end{array}$ & $\begin{array}{l}\text { rapid stop of motor; catcher } \\
\text { bearings }\end{array}$ & Vibration sensor; position sensors & $\begin{array}{l}\text { system would have to be replaced } \\
\text { and repaired (extra motor to be } \\
\text { switched in) }\end{array}$ \\
\hline & & & Crack in individual laminations & $\begin{array}{l}\text { Thermal Stresses; Latent Flaw; } \\
\text { Shearing; Fiduciary Marker } \\
\text { causes a weak point to crack } \\
\text { under stresses; }\end{array}$ & $\begin{array}{l}\text { Loss of marker showing the exact } \\
\text { position of the rotor; laminations } \\
\text { could break apart; insulation } \\
\text { failures }\end{array}$ & $\begin{array}{l}\text { reduction of motor speed; } \\
\text { potential stop for motor exchange }\end{array}$ & $\begin{array}{l}\text { position sensors; fiduciary marker } \\
\text { tracker; }\end{array}$ & $\begin{array}{l}\text { system would have to be replaced } \\
\text { and repaired (extra motor to be } \\
\text { switched in) }\end{array}$ \\
\hline & & & Detach from Axle & $\begin{array}{l}\text { Thermal Stresses; Faulty } \\
\text { Welding; Break in Laminations; } \\
\text { Excessive Vibration; Latent Flaw }\end{array}$ & $\begin{array}{l}\text { loss of rotation; impeller slowed; } \\
\text { loss of coolant flow; rotor crack; } \\
\text { can penetration; corrosion of } \\
\text { rotor; motor failure; }\end{array}$ & $\begin{array}{l}\text { rapid stop; speed reduction of } \\
\text { motor to complete stop; catcher } \\
\text { bearings }\end{array}$ & $\begin{array}{l}\text { Vibration sensor; position } \\
\text { sensors; temperature sensor; }\end{array}$ & $\begin{array}{l}\text { system would have to be replaced } \\
\text { and repaired (extra motor to be } \\
\text { switched in) }\end{array}$ \\
\hline & & & $\begin{array}{l}\text { Insulation between laminations } \\
\text { fails }\end{array}$ & Latent flaw; Exceeds temperature & $\begin{array}{l}\text { Increase in Eddy Currents; arc- } \\
\text { overs; decreased torque of motor }\end{array}$ & $\begin{array}{l}\text { reduction of motor speed; } \\
\text { potential stop for motor exchange }\end{array}$ & $\begin{array}{l}\text { Vibration sensor; temperature } \\
\text { sensor; control sensors }\end{array}$ & $\begin{array}{l}\text { system would have to be replaced } \\
\text { and repaired (extra motor to be } \\
\text { switched in) }\end{array}$ \\
\hline & & & $\begin{array}{l}\text { Not respond to Outer magnetic } \\
\text { Bearing Laminations }\end{array}$ & $\begin{array}{l}\text { Excessive Eddy currents; power } \\
\text { failure; Excessive Heat; Latent } \\
\text { Flaw ; Outer Magnetic Bearing } \\
\text { Laminations/winding failure; } \\
\text { Motor failure; Excess torque }\end{array}$ & $\begin{array}{l}\text { rotation is slowed then stopped; } \\
\text { salt flow is stopped; motor failure }\end{array}$ & $\begin{array}{l}\text { rapid stop of motor; catcher } \\
\text { bearings }\end{array}$ & position sensors failure; & $\begin{array}{l}\text { system would have to be replaced } \\
\text { and repaired (extra motor to be } \\
\text { switched in) }\end{array}$ \\
\hline
\end{tabular}


Table A-14 (continued)

\begin{tabular}{|c|c|c|c|c|c|c|c|c|}
\hline & Component & Function & Potential Failure Modes & Potential Cause of Failure & $\begin{array}{l}\text { Potential Effect of Failure } \\
\text { Mode }\end{array}$ & Control Requirement & Detection Method & Corrective Action \\
\hline & $\begin{array}{l}\text { Trapped Gas Inside } \\
\text { Rotor (Argon?) }\end{array}$ & $\begin{array}{l}\text { Replace any oxygen inside motor, } \\
\text { is a neutral gas and less } \\
\text { dangerous in the conditions of the } \\
\text { motor. }\end{array}$ & leak into environment & $\begin{array}{l}\text { rotor can failure and stator can } \\
\text { failure; seal failure; }\end{array}$ & $\begin{array}{l}\text { oxygen could make its way into } \\
\text { motor causing oxidation of } \\
\text { components; escaping into } \\
\text { environment; corrosion of motor; } \\
\text { motor speed reduction }\end{array}$ & $\begin{array}{l}\text { rapid stop of motor; catcher } \\
\text { bearings }\end{array}$ & $\begin{array}{l}\text { motor speed sensor; vibration } \\
\text { sensor; }\end{array}$ & $\begin{array}{l}\text { this is also a can failure so motor } \\
\text { would have to be replaced }\end{array}$ \\
\hline & Rotor Potting & Structure, Heat Transfer & Cracks & \begin{tabular}{|l} 
Corrosion; fatigue; excessive \\
pressure; excessive vibration; \\
thermal stresses; material fatigue \\
due to linear thermal expansion
\end{tabular} & $\begin{array}{l}\text { loss of structure; pressure against } \\
\text { rotor; }\end{array}$ & $\begin{array}{l}\text { reduction of motor speed; stop for } \\
\text { motor exchange; catcher bearings }\end{array}$ & $\begin{array}{l}\text { temperature sensor; pressure } \\
\text { noticed in control loop; }\end{array}$ & $\begin{array}{l}\text { system would have to be replaced } \\
\text { and repaired (extra motor to be } \\
\text { switched in) }\end{array}$ \\
\hline & Rotor Can & $\begin{array}{l}\text { Contain salt from corroding } \\
\text { Rotor and rotor components }\end{array}$ & Leakage of salt into Rotor & $\begin{array}{l}\text { Corrosion over a long period of } \\
\text { time; excessive pressure; } \\
\text { excessive vibration; shearing } \\
\text { stress; fatiguee, rubbing; ;in-hole } \\
\text { leaks; weld failure; thermal } \\
\text { stresses }\end{array}$ & Corrosion of rotor; motor failure & $\begin{array}{l}\text { rapid stop of motor; catcher } \\
\text { bearings }\end{array}$ & $\begin{array}{l}\text { position sensors; flow meter } \\
\text { decreasing }\end{array}$ & $\begin{array}{l}\text { system would have to be replaced } \\
\text { and repaired (extra motor to be } \\
\text { switched in) }\end{array}$ \\
\hline \multirow{3}{*}{ 壳 } & \multirow[t]{3}{*}{ FLiNaK } & \multirow[t]{3}{*}{$\begin{array}{l}\text { Coolant of Reactor. Liquid used } \\
\text { to rotate impeller and maintains } \\
\text { drive rotor temperature in normal } \\
\text { range }\end{array}$} & Solidifying & $\begin{array}{l}\text { Drive Stator Heating/Cooling } \\
\text { failure; }\end{array}$ & $\begin{array}{l}\text { Fluoride Gas Emission; } \\
\text { Rotor/stator can penetration }\end{array}$ & reduction of flow of coolant; & $\begin{array}{l}\text { flow meter; positional sensors; } \\
\text { temperature sensors before and } \\
\text { after motor }\end{array}$ & $\begin{array}{l}\text { heating up system by applying } \\
\text { more power or replacing system }\end{array}$ \\
\hline & & & Cooling flow is reduced & $\begin{array}{l}\text { Blockage of cooling channel } \\
\text { (partiai or full); loss of salt head } \\
\text { pressure; vapor lock }\end{array}$ & $\begin{array}{l}\text { pressure on can (potential leakage } \\
\text { cause); stalling of motor; }\end{array}$ & $\begin{array}{l}\text { reduced flow of coolant into } \\
\text { motor; reduction of motor speed }\end{array}$ & flow meter; positional sensors & $\begin{array}{l}\text { potential stop and restart of } \\
\text { system after a check up to make } \\
\text { sure nothing is blocking flow }\end{array}$ \\
\hline & & & No salt & blocked entrance; vapor lock & $\begin{array}{l}\text { pressure on can (potential leakage } \\
\text { cause); stalling of motor; }\end{array}$ & $\begin{array}{l}\text { reduction of motor speed; able to } \\
\text { switch to start up mode; }\end{array}$ & $\begin{array}{l}\text { flow meter; positional } \\
\text { sensors(lack of pressure will } \\
\text { throw system off) }\end{array}$ & $\begin{array}{l}\text { Switch system to start up mode } \\
\text { until salt flow can be started again } \\
\text { or motor completely stopepd. }\end{array}$ \\
\hline 8 & Catcher Bearings & $\begin{array}{l}\text { Prevent Stator/Rotor cans from } \\
\text { colliding during extreme } \\
\text { moments. }\end{array}$ & mechanical failure & $\begin{array}{l}\text { Corrosion over time; load exceeds } \\
\text { limits; not able to slowly release } \\
\text { can onto touchdown(too much } \\
\text { friction) }\end{array}$ & $\begin{array}{l}\text { Do not prevent rotor from making } \\
\text { contact with stator }\end{array}$ & none & none & $\begin{array}{l}\text { this would destroy a large part of } \\
\text { the motor a replacement might be } \\
\text { possible though }\end{array}$ \\
\hline
\end{tabular}


Table A-14 (continued)

\begin{tabular}{|c|c|c|c|c|c|c|c|c|}
\hline & Component & Function & Potential Failure Modes & Potential Cause of Failure & $\begin{array}{l}\text { Potential Effect of Failure } \\
\text { Mode }\end{array}$ & Control Requirement & Detection Method & Corrective Action \\
\hline \multirow{7}{*}{ 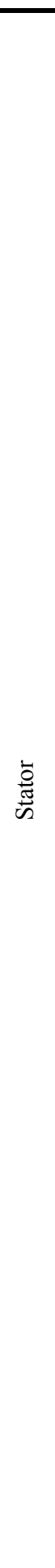 } & Stator Can & $\begin{array}{l}\text { Contain salt from corroding } \\
\text { Stator and stator components }\end{array}$ & Crack (Leaking) & $\begin{array}{l}\text { Corrosion over a long period of } \\
\text { time; excessive pressure; } \\
\text { excessive vibration; shearing } \\
\text { stress; fatigue; contact with stator; } \\
\text { welding fail; pin-hole leaks; } \\
\text { rubbing }\end{array}$ & $\begin{array}{l}\text { Corrosion of stator windings; loss } \\
\text { of control }\end{array}$ & $\begin{array}{l}\text { rapid stop of motor; catcher } \\
\text { bearings }\end{array}$ & $\begin{array}{l}\text { position sensors; flow meter } \\
\text { decreasing; leak detected }\end{array}$ & $\begin{array}{l}\text { system would have to be replaced } \\
\text { and repaired (extra motor to be } \\
\text { switched in) }\end{array}$ \\
\hline & \multirow[t]{4}{*}{$\begin{array}{l}\text { Stator Laminations } \\
\text { (Outer Magnetic } \\
\text { Bearing Laminations) }\end{array}$} & \multirow[t]{4}{*}{ Provide a path for magnetic flux } & Break Apart or Yolk break & $\begin{array}{l}\text { Thermal Stresses; Faulty } \\
\text { Welding; Latent Flaw; Shearing; } \\
\text { Crack in a single Lamination }\end{array}$ & $\begin{array}{l}\text { flux goes on incorrect path; motor } \\
\text { failure }\end{array}$ & $\begin{array}{l}\text { rapid stop of motor; catcher } \\
\text { bearings }\end{array}$ & Current signature & $\begin{array}{l}\text { system would have to be replaced } \\
\text { and repaired (extra motor to be } \\
\text { switched in) }\end{array}$ \\
\hline & & & Crack in individual laminations & $\begin{array}{l}\text { Thermal Stresses; Latent Flaw; } \\
\text { Shearing; }\end{array}$ & $\begin{array}{l}\text { laminations could break apart; } \\
\text { insulation failures; flux goes on } \\
\text { incorrect path; motor failure }\end{array}$ & Limit forces generated & Current signature & $\begin{array}{l}\text { system would have to be replaced } \\
\text { and repaired (extra motor to be } \\
\text { switched in) }\end{array}$ \\
\hline & & & $\begin{array}{l}\text { Insulation between laminations } \\
\text { fails }\end{array}$ & Latent flaw; Exceeds temperature & $\begin{array}{l}\text { Increase in Eddy Currents; arc- } \\
\text { overs; decreased torque of motor }\end{array}$ & Instigate voltage limit & $\begin{array}{l}\text { Current signature; temperature } \\
\text { measurement; partial discharge } \\
\text { detection; voltage measurement }\end{array}$ & $\begin{array}{l}\text { system would have to be replaced } \\
\text { and repaired (extra motor to be } \\
\text { switched in) }\end{array}$ \\
\hline & & & $\begin{array}{l}\text { Bearing not responding to } \\
\text { windings }\end{array}$ & $\begin{array}{l}\text { Excessive Eddy currents; power } \\
\text { failur; Excessive Heat; Outer } \\
\text { Magnetic Bearing } \\
\text { Laminations/winding failure; } \\
\text { Motor failure }\end{array}$ & \begin{tabular}{|l|} 
Loss of position control \\
\end{tabular} & $\begin{array}{l}\text { Devolve to bearing control with } \\
\text { less than full complement of } \\
\text { windings }\end{array}$ & $\begin{array}{l}\text { Current measurement; } \\
\text { observation of secondary position } \\
\text { measurement }\end{array}$ & $\begin{array}{l}\text { system would have to be replaced } \\
\text { and repaired (extra motor to be } \\
\text { switched in) }\end{array}$ \\
\hline & Stator Windings & $\begin{array}{l}\text { Circumferentially sequence } \\
\text { magnetic field }\end{array}$ & $\begin{array}{l}\text { Winding material melts } \\
\text { (Diffusion of silver into copper) }\end{array}$ & Over temperature; & increase resistivity & $\begin{array}{l}\text { Control current to limit value } \\
\text { depending on severity }\end{array}$ & Current waveform analysis & $\begin{array}{l}\text { system would have to be replaced } \\
\text { and repaired (extra motor to be } \\
\text { switched in) }\end{array}$ \\
\hline & Stator Potting & $\begin{array}{l}\text { Structure, Heat Transfer, Support } \\
\text { Windings (Prevent arc over) }\end{array}$ & Cracks & $\begin{array}{l}\text { Corrosion; fatigue; excessive } \\
\text { pressure, excessive vibration; } \\
\text { over temperature; thermal } \\
\text { stresses; material fatigue due to } \\
\text { linear thermal expansion }\end{array}$ & $\begin{array}{l}\text { loss of structure; pressure against } \\
\text { stator; arc overs; reduction of } \\
\text { torque; temperature sensors } \\
\text { disrupted }\end{array}$ & $\begin{array}{l}\text { Control currents to Limit heat } \\
\text { generation }\end{array}$ & $\begin{array}{l}\text { Continuous analysis of } \\
\text { temperature history }\end{array}$ & $\begin{array}{l}\text { system would have to be replaced } \\
\text { and repaired (extra motor to be } \\
\text { switched in) }\end{array}$ \\
\hline
\end{tabular}


Table A-14 (continued)

\begin{tabular}{|c|c|c|c|c|c|c|c|c|}
\hline & Component & Function & Potential Failure Modes & Potential Cause of Failure & $\begin{array}{l}\text { Potential Effect of Failure } \\
\text { Mode }\end{array}$ & Control Requirement & Detection Method & Corrective Action \\
\hline \multirow{4}{*}{ 产 } & Support Structure & $\begin{array}{l}\text { Adds extra support to mechanical } \\
\text { system and touchdown bearings. } \\
\text { Also helps with the excess } \\
\text { vibrations of the system by } \\
\text { making it more stable }\end{array}$ & $\begin{array}{l}\text { Detachment from stator } \\
\text { laminations }\end{array}$ & $\begin{array}{l}\text { Hardware failure; latent flaw; } \\
\text { Thermal Stresses }\end{array}$ & Eventual motor failure & Reduce motor rpm & Vibration; current signatures & $\begin{array}{l}\text { system would have to be replaced } \\
\text { and repaired (extra motor to be } \\
\text { switched in) }\end{array}$ \\
\hline & & & Fails to support & $\begin{array}{l}\text { Hardware failure; latent flaw; } \\
\text { Thermal Stresses; pressure } \\
\text { increase exceeds maximum } \\
\text { design value }\end{array}$ & failure of motor & Stop motor & Vibration & $\begin{array}{l}\text { system would have to be replaced } \\
\text { and repaired (extra motor to be } \\
\text { switched in) }\end{array}$ \\
\hline & Insulation & $\begin{array}{l}\text { Resists the flow of heat from } \\
\text { motor to outside environment } \\
\text { (like an oven mitt) }\end{array}$ & loss of insulation & $\begin{array}{l}\text { excessive heating; human error } \\
\text { (removal, rubbing etc.) }\end{array}$ & $\begin{array}{l}\text { Shorten life of windings or } \\
\text { peripheral components }\end{array}$ & & Temperature measurement & $\begin{array}{l}\text { system would have to be replaced } \\
\text { and repaired (extra motor to be } \\
\text { switched in) }\end{array}$ \\
\hline & Safety Cage & $\begin{array}{l}\text { Limits approach to motor } \\
\text { assembly without impairing } \\
\text { cooling air flow }\end{array}$ & Detachment & $\begin{array}{l}\text { Latent flaw; excessive vibration; } \\
\text { maintenance error }\end{array}$ & $\begin{array}{l}\text { Maintenance equipment could } \\
\text { impact motor assembly }\end{array}$ & None & Visual inspection & $\begin{array}{l}\text { system would have to be replaced } \\
\text { and repaired (extra motor to be } \\
\text { switched in) }\end{array}$ \\
\hline \multirow{4}{*}{ 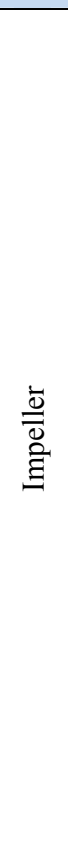 } & Impeller & $\begin{array}{l}\text { Accelerating the fluid outwards } \\
\text { from the center of rotation } \\
\text { transferring energy from the } \\
\text { motor that drives the pump to the } \\
\text { FLiNaK being pumped }\end{array}$ & Detaches from axle & $\begin{array}{l}\text { Thermal Stresses; Shearing; } \\
\text { Break in Rotor Laminations; } \\
\text { Latent Flaw; Excessive } \\
\text { Vibrations; }\end{array}$ & $\begin{array}{l}\text { Crashes into Impeller Casing; salt } \\
\text { flow stops; motor failure; }\end{array}$ & Stop motor & Vibration; loss of torque & $\begin{array}{l}\text { system would have to be replaced } \\
\text { and repaired (extra motor to be } \\
\text { switched in) }\end{array}$ \\
\hline & & & Does not rotate & $\begin{array}{l}\text { Rotor/Axle fail; Stopped coolant } \\
\text { flow; solidified coolant; ; ver } \\
\text { torque; loss of control system }\end{array}$ & salt flow stops; & Stop motor & Current measurement & $\begin{array}{l}\text { system would have to be replaced } \\
\text { and repaired (extra motor to be } \\
\text { switched in) }\end{array}$ \\
\hline & \multirow[t]{2}{*}{ Impeller Casing } & $\begin{array}{l}\text { Contain the flow of salt and } \\
\text { Impeller }\end{array}$ & Leaks & $\begin{array}{l}\text { Corrosion; pin-hole leaks; } \\
\text { welding fail; seal broken; } \\
\text { excessive pressure; Cavitation }\end{array}$ & $\begin{array}{l}\text { corrosion of support structure; } \\
\text { salt in the environment; flow of } \\
\text { salt slowed }\end{array}$ & None & None & $\begin{array}{l}\text { system would have to be replaced } \\
\text { and repaired (extra motor to be } \\
\text { switched in) }\end{array}$ \\
\hline & & & Fail to support & $\begin{array}{l}\text { Corrosion; too much pressure at } \\
\text { once }\end{array}$ & failure of impeller & & & \\
\hline
\end{tabular}


Table A-14 (continued)

\begin{tabular}{|c|c|c|c|c|c|c|c|c|}
\hline & Component & Function & Potential Failure Modes & Potential Cause of Failure & $\begin{array}{c}\text { Potential Effect of Failure } \\
\text { Mode }\end{array}$ & Control Requirement & Detection Method & Corrective Action \\
\hline \multirow{2}{*}{ 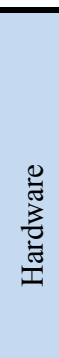 } & Bolts & $\begin{array}{l}\text { Connect the parts of the motor } \\
\text { together (i.e. the stator rotor etc.) }\end{array}$ & Break or loosen & $\begin{array}{l}\text { latent flaw; exceed temperature, } \\
\text { vibration, improperly tightened; } \\
\text { no thread lock }\end{array}$ & \begin{tabular}{|l|} 
Detachment of support structure, \\
rotor, stator, etc.; vibration;
\end{tabular} & $\begin{array}{l}\text { Reduce motor rpm depending on } \\
\text { severity }\end{array}$ & Vibration & replacement of bolts; \\
\hline & \begin{tabular}{|l|} 
Seals \\
\end{tabular} & $\begin{array}{l}\begin{array}{l}\text { Join parts of motor together by } \\
\text { preventing leakage of salt }\end{array} \\
\end{array}$ & Leakage & $\begin{array}{l}\text { wears overtime; thermal stresses; } \\
\text { improper installation; corrosion }\end{array}$ & corrosion of motor & None & None directly & $\begin{array}{l}\text { replacement of seals; layered } \\
\text { seals }\end{array}$ \\
\hline
\end{tabular}


Table A-15. Electronic system

\begin{tabular}{|c|c|c|c|c|c|c|c|c|}
\hline Component & Function & Potential Failure Modes & Potential Cause of Failure & $\begin{array}{l}\text { Potential Effect of Failure } \\
\text { Mode }\end{array}$ & Control Requirement & Detection Method & Corrective Action & Defense in Depth \\
\hline \multirow[t]{3}{*}{ Control Power Electronics } & \multirow[t]{3}{*}{ Supply power to control system } & Loss of power & $\begin{array}{l}\text { Blown fuse or circuit } \\
\text { breaker; failed components; } \\
\text { over-heating; human error } \\
\text { (accidental unplugging); } \\
\text { short circuit; open circuit }\end{array}$ & Motor stops & Shut down drivers & Self monitoring & Repair & $\begin{array}{l}\text { Redundant power supplies; } \\
\text { redundant drive electronics }\end{array}$ \\
\hline & & Drop in Voltage & $\begin{array}{l}\text { Failed components; over- } \\
\text { heating; power surge; short } \\
\text { circuit; grounding issues; } \\
\text { brown out }\end{array}$ & $\begin{array}{l}\text { Degraded performance; } \\
\text { Reduced torque }\end{array}$ & $\begin{array}{l}\text { Change torque management } \\
\text { or rpm to accommodate } \\
\text { voltage drop until recovered }\end{array}$ & Self monitoring & Repair & $\begin{array}{l}\text { Redundant power supplies; } \\
\text { redundant drive electronics }\end{array}$ \\
\hline & & Intermittent voltage & $\begin{array}{l}\text { Failed components; over- } \\
\text { heating; power surge; short } \\
\text { circuit; grounding issues; }\end{array}$ & Intermittent speed control & $\begin{array}{l}\text { Change torque management } \\
\text { or rpm to accommodate } \\
\text { voltage drop until recovered }\end{array}$ & Self monitoring & Troubleshoot and repair & $\begin{array}{l}\text { Redundant power supplies; } \\
\text { redundant drive electronics }\end{array}$ \\
\hline \multirow[t]{3}{*}{ Power Supplies } & \multirow[t]{3}{*}{$\begin{array}{l}\text { Provides DC voltage to drive } \\
\text { electronics }\end{array}$} & Loss of Power & $\begin{array}{l}\text { Blown fuse or circuit } \\
\text { breaker; failed components; } \\
\text { over-heating; human error } \\
\text { (accidental unplugging); } \\
\text { interlock protection } \\
\text { activated; short circuit; } \\
\text { open circuit; }\end{array}$ & Motor stops & Shut down drivers & Self monitoring & & $\begin{array}{l}\text { Use burn-in process on } \\
\text { individual components; }\end{array}$ \\
\hline & & Drop in supply voltage & $\begin{array}{l}\text { failed components; over- } \\
\text { heating; brown out; } \\
\text { grounding issues }\end{array}$ & $\begin{array}{l}\text { Degraded performance; } \\
\text { reduced torque }\end{array}$ & $\begin{array}{l}\text { Change torque management } \\
\text { or rpm to accommodate } \\
\text { voltage drop until recovered }\end{array}$ & Self monitoring & & $\begin{array}{l}\text { Use burn-in process on } \\
\text { individual components; }\end{array}$ \\
\hline & & Intermittent voltage & $\begin{array}{l}\text { failed components; over- } \\
\text { heating; human error } \\
\text { (accidental unplugging and } \\
\text { corrective replugging); } \\
\text { grounding issues }\end{array}$ & Reduced torque & $\begin{array}{l}\text { Change torque management } \\
\text { or rpm to accommodate } \\
\text { voltage drop until recovered }\end{array}$ & Self monitoring & & $\begin{array}{l}\text { Use burn-in process on } \\
\text { individual components; }\end{array}$ \\
\hline
\end{tabular}


Table A-15 (continued)

\begin{tabular}{|c|c|c|c|c|c|c|c|c|}
\hline Component & Function & Potential Failure Modes & Potential Cause of Failure & $\begin{array}{l}\text { Potential Effect of Failure } \\
\text { Mode }\end{array}$ & Control Requirement & Detection Method & Corrective Action & Defense in Depth \\
\hline \multirow[t]{4}{*}{\begin{tabular}{|l} 
Motor Power Electronics \\
\end{tabular}} & \multirow[t]{4}{*}{$\begin{array}{l}\text { Converts ac to dc and provides } \\
\text { modulated current to poly-phase } \\
\text { stator windings }\end{array}$} & Single Phase Open Circuit & $\begin{array}{l}\text { Failure of a single } \\
\text { transistor; switch-on failure } \\
\text { of both transistors on one } \\
\text { leg; break down of one } \\
\text { phase leg; latent flaw }\end{array}$ & $\begin{array}{l}\text { Increased torque ripple; } \\
\text { motor vibration; loss of } \\
\text { torque }\end{array}$ & $\begin{array}{l}\text { Switch to degraded control } \\
\text { mode in which not all } \\
\text { windings are driven }\end{array}$ & $\begin{array}{l}\text { Monitoring of individual } \\
\text { phase currents }\end{array}$ & Repair or replace motor & \\
\hline & & Single Phase Short Circuit & $\begin{array}{l}\text { switch off failure of one } \\
\text { transistor; ground of single } \\
\text { motor phase; latent flaw; } \\
\text { failed components; over } \\
\text { heating; safety protection } \\
\text { activated; human error }\end{array}$ & $\begin{array}{l}\text { Blown fuse or circuit } \\
\text { breaker; }\end{array}$ & $\begin{array}{l}\text { Switch to degraded control } \\
\text { mode in which not all } \\
\text { windings are driven }\end{array}$ & $\begin{array}{l}\text { Monitoring of individual } \\
\text { phase currents }\end{array}$ & Repair or replace motor & \\
\hline & & Three Phase Short Circuit & $\begin{array}{l}\text { switches permanently } \\
\text { closed; ground of } 3 \text { phases; } \\
\text { packaging failure } \\
\text { (overstress on wire bonds; } \\
\text { thermal expansion; } \\
\text { exceeding temperature } \\
\text { range); latent flaw }\end{array}$ & Motor stops & Shut down motor & $\begin{array}{l}\text { Monitoring of individual } \\
\text { phase currents }\end{array}$ & Repair or replace motor & \\
\hline & & Total loss of drive current & $\begin{array}{l}\text { Blown fuse or circuit } \\
\text { breaker, failed components; } \\
\text { over heating; safety } \\
\text { protection activated; human } \\
\text { error; }\end{array}$ & Motor stops & Shut down motor & $\begin{array}{l}\text { Monitoring of individual } \\
\text { phase currents }\end{array}$ & Repair or replace motor & $\begin{array}{l}\text { Use burn-in process on } \\
\text { individual components; }\end{array}$ \\
\hline \multirow[t]{2}{*}{\begin{tabular}{|l} 
Windings \\
\end{tabular}} & \multirow[t]{2}{*}{$\begin{array}{l}\text { Circumferentially sequence } \\
\text { magnetic field }\end{array}$} & Short Circuit & $\begin{array}{l}\text { Turn to Turn, coil to coil, } \\
\text { phase to phase, coil to } \\
\text { ground short Circuit; latent } \\
\text { flaw }\end{array}$ & $\begin{array}{l}\text { Motor torque adversely } \\
\text { affected; loss of control; } \\
\text { blow fuses/breakers }\end{array}$ & $\begin{array}{l}\text { Switch to degraded control } \\
\text { mode in which not all } \\
\text { windings are driven }\end{array}$ & $\begin{array}{l}\text { Monitor current and breaker } \\
\text { status }\end{array}$ & Repair or replace motor & \\
\hline & & Open Circuit & $\begin{array}{l}\text { Open Circuit; latent flaw; } \\
\text { mechanical failure }\end{array}$ & $\begin{array}{l}\text { Motor torque adversely } \\
\text { affected; loss of control; }\end{array}$ & $\begin{array}{l}\text { Switch to degraded control } \\
\text { mode in which not all } \\
\text { windings are driven }\end{array}$ & $\begin{array}{l}\text { Monitor current and breaker } \\
\text { status }\end{array}$ & Repair or replace motor & \\
\hline \begin{tabular}{|l} 
Wire Leads \\
\end{tabular} & $\begin{array}{l}\text { Electrically connecting devices to } \\
\text { supply power and current }\end{array}$ & Open/short Circuit & $\begin{array}{l}\text { Excessive current; Metal } \\
\text { fatigue; eroded wire frames; } \\
\text { corrosion; improper } \\
\text { rapping; over temperature; } \\
\text { latent flaw; Excessive } \\
\text { vibration; short; }\end{array}$ & $\begin{array}{l}\text { Motor torque adversely } \\
\text { affected; loss of control; }\end{array}$ & $\begin{array}{l}\text { Switch to degraded control } \\
\text { mode in which not all } \\
\text { windings are driven }\end{array}$ & $\begin{array}{l}\text { Monitor current and breaker } \\
\text { status }\end{array}$ & Repair or replace motor & \\
\hline
\end{tabular}


Table A-16. Control system

\begin{tabular}{|c|c|c|c|c|c|c|c|c|c|}
\hline & Component & Function & Potential Failure Modes & $\begin{array}{l}\text { Potential Cause of } \\
\text { Failure }\end{array}$ & $\begin{array}{l}\text { Potential Effect of Failure } \\
\text { Mode }\end{array}$ & Control Requirement & Detection Method & Corrective Action & Defense in Depth \\
\hline \multirow{4}{*}{ 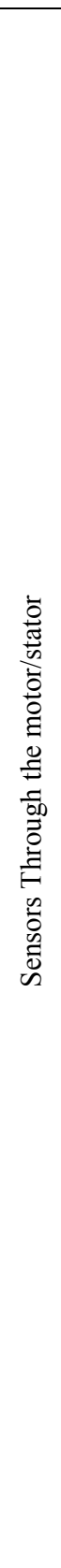 } & \multirow[t]{2}{*}{ Rotational Position } & \multirow[t]{2}{*}{$\begin{array}{l}\text { Positional sensor through the } \\
\text { stator that reads the position of } \\
\text { the rotation of the rotor system. }\end{array}$} & No reading & $\begin{array}{l}\text { Open wire; mechanical } \\
\text { failure; incorrect frequency } \\
\text { setting (reads that it doesn't } \\
\text { even exist); active } \\
\text { frequency }\end{array}$ & $\begin{array}{l}\text { no rotational position data } \\
\text { for control algorithms (no } \\
\text { speed data if derived); } \\
\text { unknown rotational } \\
\text { position; }\end{array}$ & $\begin{array}{l}\text { Switch control algorithm to } \\
\text { accommodate alternate } \\
\text { measurements; shut down } \\
\text { motor if no measurement is } \\
\text { possible }\end{array}$ & \begin{tabular}{|l|} 
Compare independent \\
sensors with sensorless \\
(calculated) values
\end{tabular} & Alert operator and repair & \\
\hline & & & Inaccurate readings & $\begin{array}{l}\text { Noise; drift error; } \\
\text { interference (from other } \\
\text { sensors frequencies or } \\
\text { suspension current); high } \\
\text { temperature drift; exceeded } \\
\text { limits; low rotational speed } \\
\text { reading might have error }\end{array}$ & $\begin{array}{l}\text { incorrect data for } \\
\text { algorithms; }\end{array}$ & $\begin{array}{l}\text { Switch control algorithm to } \\
\text { accommodate alternate } \\
\text { measurements; shut down } \\
\text { motor if no measurement is } \\
\text { possible }\end{array}$ & \begin{tabular}{|l} 
Compare independent \\
sensors with sensorless \\
(calculated) values
\end{tabular} & Alert operator and repair & \\
\hline & \multirow[t]{2}{*}{ Rotational Speed } & \multirow[t]{2}{*}{$\begin{array}{l}\text { Positional sensor through the } \\
\text { stator that reads the speed of } \\
\text { the rotations. }\end{array}$} & No reading & $\begin{array}{l}\text { Open wire; mechanical } \\
\text { failure; incorrect frequency } \\
\text { setting (reads that it doesn't } \\
\text { even exist);active } \\
\text { frequency }\end{array}$ & $\begin{array}{l}\text { no data for algorithms; no } \\
\text { known rotational speed; }\end{array}$ & $\begin{array}{l}\text { Switch control algorithm to } \\
\text { accommodate alternate } \\
\text { measurements; shut down } \\
\text { motor if no measurement is } \\
\text { possible }\end{array}$ & \begin{tabular}{|l} 
Compare independent \\
sensors with sensorless \\
(calculated) values
\end{tabular} & Alert operator and repair & \\
\hline & & & Inaccurate readings & $\begin{array}{l}\text { Noise; drift error; } \\
\text { interference (from other } \\
\text { sensors frequencies or } \\
\text { suspension current); high } \\
\text { temperature drift; } \\
\text { calculation incorrect } \\
\text { (integration drift); } \\
\text { exceeded limits }\end{array}$ & $\begin{array}{l}\text { incorrect data for } \\
\text { algorithms; }\end{array}$ & $\begin{array}{l}\text { Switch control algorithm to } \\
\text { accommodate alternate } \\
\text { measurements; shut down } \\
\text { motor if no measurement is } \\
\text { possible }\end{array}$ & \begin{tabular}{|l} 
Compare independent \\
sensors with sensorless \\
(calculated) values
\end{tabular} & Alert operator and repair & \\
\hline
\end{tabular}


Table A-16 (continued)

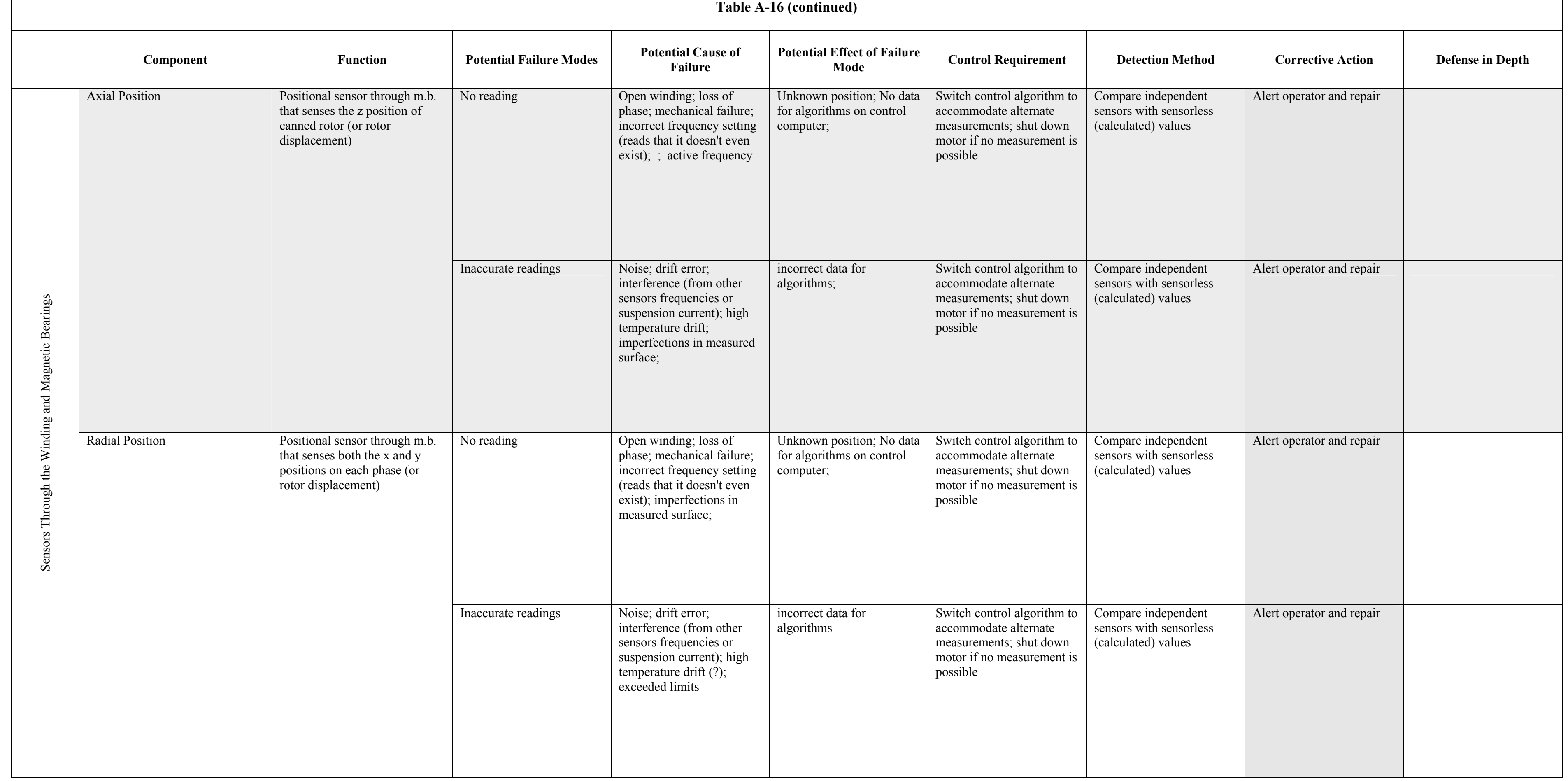


Table A-16 (continued)

\begin{tabular}{|c|c|c|c|c|c|c|c|c|c|}
\hline \multicolumn{10}{|c|}{ Table A-16 (continued) } \\
\hline & Component & Function & Potential Failure Modes & $\begin{array}{l}\text { Potential Cause of } \\
\text { Failure }\end{array}$ & $\begin{array}{l}\text { Potential Effect of Failure } \\
\text { Mode }\end{array}$ & Control Requirement & Detection Method & Corrective Action & Defense in Depth \\
\hline \multirow{6}{*}{ 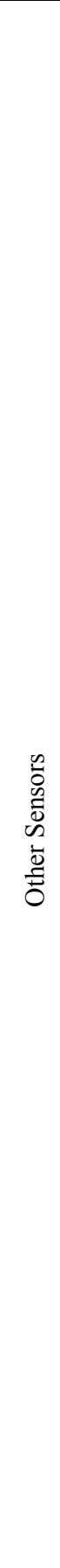 } & \multirow[t]{2}{*}{ Temperature (Interior) } & \multirow[t]{2}{*}{$\begin{array}{l}\text { Monitors the temperature of the } \\
\text { windings and interior parts of } \\
\text { motor }\end{array}$} & No reading & $\begin{array}{l}\text { open wire; mechanical } \\
\text { failure (spot welding of } \\
\text { thermal coupling failure; } \\
\text { grounding issues to the } \\
\text { sheaf) ; excessive } \\
\text { movement or stress; } \\
\text { oxidation of thermal couple }\end{array}$ & $\begin{array}{l}\text { Overheating; no data for } \\
\text { algorithms; overcooling; }\end{array}$ & $\begin{array}{l}\text { Adjust control until sensor } \\
\text { can be repaired }\end{array}$ & $\begin{array}{l}\text { Estimate temperature from } \\
\text { dynamic current and } \\
\text { voltage measurements }\end{array}$ & Alert operator and repair & \\
\hline & & & Inaccurate readings & $\begin{array}{l}\text { improper offset; noise; } \\
\text { exceeded limits; } \\
\text { interference }\end{array}$ & $\begin{array}{l}\text { incorrect data for } \\
\text { algorithms; }\end{array}$ & $\begin{array}{l}\text { Adjust control until sensor } \\
\text { can be repaired }\end{array}$ & $\begin{array}{l}\text { Estimate temperature from } \\
\text { dynamic current and } \\
\text { voltage measurements }\end{array}$ & Alert operator and repair & \\
\hline & \multirow[t]{2}{*}{ Vibration (Exterior) } & \multirow[t]{2}{*}{$\begin{array}{l}\text { Possible sensor located on the } \\
\text { outside of motor to track the } \\
\text { overall vibration levels of the } \\
\text { motor }\end{array}$} & No reading & $\begin{array}{l}\text { open wire; mechanical } \\
\text { failure; excessive } \\
\text { movement or stress; over } \\
\text { temperature }\end{array}$ & $\begin{array}{l}\text { Excessive vibrations; no } \\
\text { data for algorithms; } \\
\text { upsetting other data; }\end{array}$ & None & $\begin{array}{l}\text { Normal vibration signature } \\
\text { is not found }\end{array}$ & Alert operator and repair & \\
\hline & & & Inaccurate readings & $\begin{array}{l}\text { excessive vibration; noise; } \\
\text { exceeded limits; drift error; } \\
\text { change in wire sensitivity } \\
\text { due to heat; }\end{array}$ & $\begin{array}{l}\text { incorrect data for } \\
\text { algorithms; excessive } \\
\text { vibrations; }\end{array}$ & None & $\begin{array}{l}\text { Normal vibration signature } \\
\text { is not correct }\end{array}$ & Alert operator and repair & \\
\hline & \multirow[t]{2}{*}{ Current (On circuitry) } & \multirow[t]{2}{*}{$\begin{array}{l}\text { Measures the current going into } \\
\text { circuitry. }\end{array}$} & No reading & 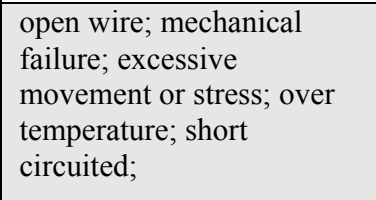 & $\begin{array}{l}\text { excessive current; no data } \\
\text { for algorithms calculations; }\end{array}$ & None & \begin{tabular}{|l|} 
Current monitored \\
independently by driver \\
electronics and secondary \\
electronics
\end{tabular} & Alert operator and repair & \\
\hline & & & Inaccurate readings & $\begin{array}{l}\text { excessive current; noise; } \\
\text { exceeded limits; drift error; } \\
\text { change in wire sensitivity } \\
\text { due to heat; }\end{array}$ & $\begin{array}{l}\text { incorrect data for } \\
\text { algorithms; excessive } \\
\text { current; }\end{array}$ & None & $\begin{array}{l}\text { Current monitored } \\
\text { independently by driver } \\
\text { electronics and secondary } \\
\text { electronics }\end{array}$ & Alert operator and repair & \\
\hline
\end{tabular}


Table A-16 (continued)

\begin{tabular}{|c|c|c|c|c|c|c|c|c|}
\hline Component & Function & Potential Failure Modes & $\begin{array}{l}\text { Potential Cause of } \\
\text { Failure }\end{array}$ & $\begin{array}{l}\text { Potential Effect of Failure } \\
\text { Mode }\end{array}$ & Control Requirement & Detection Method & Corrective Action & Defense in Depth \\
\hline \multirow[t]{2}{*}{ Flow meter (Exterior) } & \multirow[t]{2}{*}{$\begin{array}{l}\text { Uses Doppler Velocimetry to } \\
\text { see the velocity of fluid }\end{array}$} & No feedback & $\begin{array}{l}\text { Over heating; broken } \\
\text { clamp; acoustic waveguide } \\
\text { failure }\end{array}$ & $\begin{array}{l}\text { no flow data for } \\
\text { algorithms; }\end{array}$ & None & $\begin{array}{l}\text { Compare motor speed with } \\
\text { flow meter readings }\end{array}$ & Alert operator and repair & \\
\hline & & \begin{tabular}{|l} 
Inaccurate readings \\
\end{tabular} & $\begin{array}{l}\text { Offset waveguide; } \\
\text { incorrect frequency; } \\
\text { acoustic waveguide failure } \\
\text { (thermal contact in } \\
\text { between); }\end{array}$ & $\begin{array}{l}\text { incorrect flow data for } \\
\text { algorithms; }\end{array}$ & None & $\begin{array}{l}\text { Compare motor speed with } \\
\text { flow meter readings }\end{array}$ & Alert operator and repair & \\
\hline \multirow[t]{2}{*}{ Hardware Input Signal Processing } & \multirow[t]{2}{*}{$\begin{array}{l}\text { Processes signals to filter noise } \\
\text { and amplify sensor data if } \\
\text { needed. }\end{array}$} & Input not responding & $\begin{array}{l}\text { failed components; } \\
\text { grounding issues; lightning } \\
\text { discharge; computer } \\
\text { hacked; corroded } \\
\text { connections; short or open } \\
\text { wiring; }\end{array}$ & $\begin{array}{l}\text { data unable to reach control } \\
\text { computer; loss of control; }\end{array}$ & Shutdown motor & External monoitoring & Alert operator and repair & \\
\hline & & \begin{tabular}{|l} 
Incorrect signal processing \\
\end{tabular} & $\begin{array}{l}\text { cross-talk between signals; } \\
\text { drift or bias in signal value; } \\
\text { excessive noise in input }\end{array}$ & $\begin{array}{l}\text { incorrect information sent } \\
\text { to control computer; }\end{array}$ & Shutdown motor & External monoitoring & Alert operator and repair & \\
\hline \multirow[t]{3}{*}{\begin{tabular}{|l} 
Analog to Digital Converter \\
\end{tabular}} & \multirow[t]{3}{*}{$\begin{array}{l}\text { Converts analog data into } \\
\text { digital for computer usage }\end{array}$} & Incorrectly converts data & $\begin{array}{l}\text { Quantization error; } \\
\text { aperture error; component } \\
\text { failure; excessive vibration; } \\
\text { too low of a resolution; bit } \\
\text { fidelity }\end{array}$ & $\begin{array}{l}\text { incorrect information sent } \\
\text { to control computer; }\end{array}$ & $\begin{array}{l}\text { Adjust control until } \\
\text { electronics can be repaired }\end{array}$ & $\begin{array}{l}\text { Compare acquired signals } \\
\text { with model predictions }\end{array}$ & Alert operator and repair & \\
\hline & & Process is slowed down & $\begin{array}{l}\text { Quantization error; } \\
\text { aperture error; component } \\
\text { failure; excessive vibration; }\end{array}$ & $\begin{array}{l}\text { delay in control loop; } \\
\text { incorrect voltage sent } \\
\text { (overcompensate or too } \\
\text { late); }\end{array}$ & \begin{tabular}{|l|} 
Adjust control until \\
electronics can be repaired
\end{tabular} & $\begin{array}{l}\text { Compare acquired signals } \\
\text { with model predictions }\end{array}$ & Alert operator and repair & \\
\hline & & Converter not responding & $\begin{array}{l}\text { short or open wiring; } \\
\text { component failure; } \\
\text { excessive temperature; }\end{array}$ & $\begin{array}{l}\text { data unable to reach control } \\
\text { computer; loss of control; }\end{array}$ & $\begin{array}{l}\text { Adjust control until } \\
\text { electronics can be repaired }\end{array}$ & $\begin{array}{l}\text { Compare acquired signals } \\
\text { with model predictions }\end{array}$ & Alert operator and repair & \\
\hline
\end{tabular}


Table A-16 (continued)

\begin{tabular}{|c|c|c|c|c|c|c|c|c|}
\hline Component & Function & Potential Failure Modes & $\begin{array}{l}\text { Potential Cause of } \\
\text { Failure }\end{array}$ & $\begin{array}{l}\text { Potential Effect of Failure } \\
\text { Mode }\end{array}$ & Control Requirement & Detection Method & Corrective Action & Defense in Depth \\
\hline \multirow[t]{3}{*}{$\begin{array}{l}\text { Software Signal Processing } \\
\text { Algorithms }\end{array}$} & \multirow[t]{3}{*}{$\begin{array}{l}\text { a system of step-by-step } \\
\text { procedures for calculations, } \\
\text { data processing, and automated } \\
\text { reasoning of the motor control }\end{array}$} & $\begin{array}{l}\text { Incorrect algorithm } \\
\text { function }\end{array}$ & $\begin{array}{l}\text { data were not recognized } \\
\text { correctlty; incorrect code; } \\
\text { computer hacking; set } \\
\text { point decide errors; sensor } \\
\text { noise; lack of sensor data; } \\
\text { insufficiant sensor } \\
\text { resolution }\end{array}$ & $\begin{array}{l}\text { incorrect data sent to } \\
\text { control computer; too } \\
\text { much less voltage sent to } \\
\text { windings; }\end{array}$ & $\begin{array}{l}\text { Adjust control until } \\
\text { electronics can be repaired }\end{array}$ & $\begin{array}{l}\text { Motor drive signals } \\
\text { compared with simple } \\
\text { model }\end{array}$ & Alert operator and repair & \\
\hline & & loss of control & power failure of computer & motor failure; & $\begin{array}{l}\text { Adjust control until } \\
\text { electronics can be repaired }\end{array}$ & $\begin{array}{l}\text { Motor drive signals } \\
\text { compared with simple } \\
\text { model }\end{array}$ & Alert operator and repair & \\
\hline & & degraded performance & $\begin{array}{l}\text { high temperature drift not } \\
\text { taken account forget point } \\
\text { decide errors; sensor noise; } \\
\text { lack of sensor data; } \\
\text { insuffiant sensor resolution }\end{array}$ & $\begin{array}{l}\text { delay in control loop; } \\
\text { incorrect voltage sent } \\
\text { (overcompensates or too } \\
\text { late); }\end{array}$ & $\begin{array}{l}\text { Adjust control until } \\
\text { electronics can be repaired }\end{array}$ & $\begin{array}{l}\text { Motor drive signals } \\
\text { compared with simple } \\
\text { model }\end{array}$ & Alert operator and repair & \\
\hline \multirow[t]{2}{*}{$\begin{array}{l}\text { Control Computer (Software } \\
\text { Control Algorithms) }\end{array}$} & \multirow[t]{2}{*}{$\begin{array}{l}\text { Processes signals to accomplish } \\
\text { control of rotor functions under } \\
\text { all normal and abnormal } \\
\text { conditions, diagnoses current } \\
\text { signals and detects developing } \\
\text { problems, receives commands, } \\
\text { and generates status } \\
\text { information }\end{array}$} & Control not responding & $\begin{array}{l}\text { shorted or open wiring; } \\
\text { computer hacked; } \\
\text { overheated; control } \\
\text { algorithm failing; fried } \\
\text { components; power loss; } \\
\text { solar flares }\end{array}$ & motor failure; & $\begin{array}{l}\text { Divert to backup or Stop } \\
\text { motor }\end{array}$ & $\begin{array}{l}\text { Current monitored } \\
\text { independently by driver } \\
\text { electronics and secondary } \\
\text { electronics; motor drive } \\
\text { signals compared with } \\
\text { simple model }\end{array}$ & Alert operator and repair & \\
\hline & & $\begin{array}{l}\text { Incorrect drive current } \\
\text { sequence generated }\end{array}$ & $\begin{array}{l}\text { incorrect set points or } \\
\text { algorithms applied; signal } \\
\text { or command failed to } \\
\text { detect anomaly; control } \\
\text { computer hacked; output } \\
\text { validation subsystem failed } \\
\text { to detect anomalous drive; } \\
\text { solar flares }\end{array}$ & $\begin{array}{l}\text { too much/less } \\
\text { voltage/current sent to } \\
\text { motor; loss of control; } \\
\text { motor failure }\end{array}$ & $\begin{array}{l}\begin{array}{l}\text { Divert to backup or Stop } \\
\text { motor }\end{array} \\
\end{array}$ & $\begin{array}{l}\text { Current monitored } \\
\text { independently by driver } \\
\text { electronics and secondary } \\
\text { electronics; motor drive } \\
\text { signals compared with } \\
\text { simple model }\end{array}$ & Alert operator and repair & \\
\hline
\end{tabular}


Table A-16 (continued)

\begin{tabular}{|c|c|c|c|c|c|c|c|c|}
\hline Component & Function & Potential Failure Modes & $\begin{array}{l}\text { Potential Cause of } \\
\text { Failure }\end{array}$ & $\begin{array}{l}\text { Potential Effect of Failure } \\
\text { Mode }\end{array}$ & Control Requirement & Detection Method & Corrective Action & Defense in Depth \\
\hline \multirow[t]{3}{*}{$\begin{array}{l}\text { Software Output Signal } \\
\text { Conditioning }\end{array}$} & \multirow[t]{3}{*}{$\begin{array}{l}\text { Takes the control decision and } \\
\text { converts into the correct duty } \\
\text { cycle information for the PWM } \\
\text { and also potential warnings for } \\
\text { operators }\end{array}$} & $\begin{array}{l}\text { Converts control decision } \\
\text { incorrectly }\end{array}$ & $\begin{array}{l}\text { data were not recognized } \\
\text { correctly; incorrect code; } \\
\text { computer hacking; set } \\
\text { point decide errors; sensor } \\
\text { noise; lack of sensor data; } \\
\text { insufficiant sensor } \\
\text { resolution }\end{array}$ & $\begin{array}{l}\text { Incorrect power needed for } \\
\text { motor; reduced torque; } \\
\text { motor failure; }\end{array}$ & $\begin{array}{l}\text { Divert to backup or Stop } \\
\text { motor }\end{array}$ & \begin{tabular}{|l|} 
Current monitored \\
independently by driver \\
electronics and secondary \\
electronics; motor drive \\
signals compared with \\
simple model
\end{tabular} & Alert operator and repair & \\
\hline & & & power failure of computer & Motor stops & $\begin{array}{l}\text { Divert to backup or Stop } \\
\text { motor }\end{array}$ & \begin{tabular}{|l} 
Current monitored \\
independently by driver \\
electronics and secondary \\
electronics; motor drive \\
signals compared with \\
simple model
\end{tabular} & Alert operator and repair & \\
\hline & & & $\begin{array}{l}\text { high temperature drift not } \\
\text { taken account forget point } \\
\text { decide errors; sensor noise; } \\
\text { lack of sensor data; } \\
\text { insufficient sensor } \\
\text { resolution }\end{array}$ & $\begin{array}{l}\text { Unpredictable and erratic } \\
\text { motor behavior }\end{array}$ & $\begin{array}{l}\text { Divert to backup or Stop } \\
\text { motor }\end{array}$ & \begin{tabular}{|l|} 
Current monitored \\
independently by driver \\
electronics and secondary \\
electronics; motor drive \\
signals compared with \\
simple model
\end{tabular} & Alert operator and repair & \\
\hline $\begin{array}{l}\text { Hardware Output Signal } \\
\text { Processing (Pulse Width } \\
\text { Modulator) }\end{array}$ & $\begin{array}{l}\text { Controls the power to motor } \\
\text { and magnetic bearing coils by } \\
\text { using switching frequency. }\end{array}$ & Incorrect duty cycle & $\begin{array}{l}\text { failed components; } \\
\text { grounding issues; lightning } \\
\text { discharge; computer } \\
\text { hacked; corroded } \\
\text { connections; short or open } \\
\text { wiring; }\end{array}$ & $\begin{array}{l}\text { Incorrect power needed for } \\
\text { motor; reduced torque; } \\
\text { motor failure; }\end{array}$ & $\begin{array}{l}\text { Divert to backup or Stop } \\
\text { motor }\end{array}$ & \begin{tabular}{|l|} 
Current monitored \\
independently by driver \\
electronics and secondary \\
electronics; motor drive \\
signals compared with \\
simple model
\end{tabular} & Alert operator and repair & \\
\hline
\end{tabular}


Table A-16 (continued)

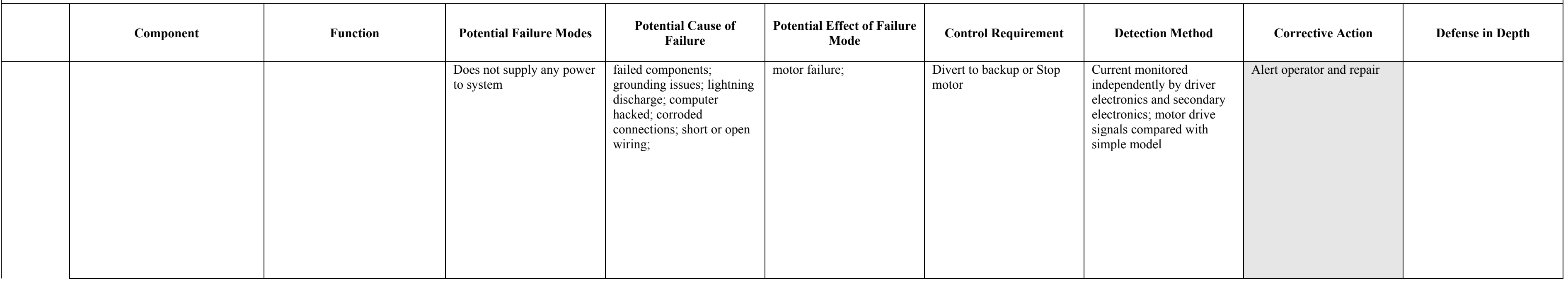


Table A-17. Bearing system components

\begin{tabular}{|c|c|c|c|c|c|c|c|c|c|}
\hline Description & Function & Failure Mode & Cause of Failure & Effect of Failure & Detection Method & Control Requirement & Corrective Action & Severity & Defense in Depth \\
\hline Bearing 1 Rotor & Maintain rotor centrality & $\begin{array}{l}\text { Rub or impact outer } \\
\text { barrier stationary can; } \\
\text { detachment from shaft }\end{array}$ & $\begin{array}{l}\text { Excessive side force; } \\
\text { corrosion; manufacturing } \\
\text { defect }\end{array}$ & $\begin{array}{l}\text { Motor failure; barrier } \\
\text { penetration }\end{array}$ & $\begin{array}{l}\text { Current signature; sensor } \\
\text { signal; vibration sensors }\end{array}$ & $\begin{array}{l}\text { Fast response; high } \\
\text { emergency drive } \\
\text { capability }\end{array}$ & Rapid stop of motor & High; rapid evolution & Multiple barrier layers \\
\hline Bearing 1 Stator & $\begin{array}{l}\text { Magnetically drive } \\
\text { bearing rotor to central } \\
\text { position }\end{array}$ & Open winding & $\begin{array}{l}\text { Over current; over } \\
\text { temperature; voltage arc- } \\
\text { over, latent flaw; } \\
\text { corrosion }\end{array}$ & $\begin{array}{l}\text { Loss of position control; } \\
\text { bearing loses holding } \\
\text { force and rotor impacts } \\
\text { stationary can }\end{array}$ & $\begin{array}{l}\text { Current monitoring, } \\
\text { voltage monitoring; } \\
\text { vibration sensors }\end{array}$ & $\begin{array}{l}\text { Diagnostics of current and } \\
\text { voltage signature }\end{array}$ & $\begin{array}{l}\text { None unless there is } \\
\text { redundant windings }\end{array}$ & High; rapid evolution & $\begin{array}{l}\text { Redundant or spare stator } \\
\text { windings }\end{array}$ \\
\hline Bearing 1 Position Sensor & $\begin{array}{l}\text { Measure X-Y shaft } \\
\text { position (multiple sensors } \\
\text { for radial centering } \\
\text { measurement) }\end{array}$ & $\begin{array}{l}\text { Wrong centering position; } \\
\text { intermittent operation; } \\
\text { dead sensor }\end{array}$ & $\begin{array}{l}\text { Over temperature; latent } \\
\text { flaw; excessive noise }\end{array}$ & $\begin{array}{l}\text { Potential loss of position } \\
\text { control }\end{array}$ & $\begin{array}{l}\text { Loss of sensor signal; } \\
\text { sensor signal not in } \\
\text { bounds of model } \\
\text { prediction; signal } \\
\text { validation methods used } \\
\text { to identify failing sensors }\end{array}$ & $\begin{array}{l}\text { Diagnostic of sensor } \\
\text { signal; alternative means } \\
\text { of determining shaft } \\
\text { position }\end{array}$ & $\begin{array}{l}\text { Switch to alternative } \\
\text { sensor method; use } \\
\text { model-based prediction; } \\
\text { plan for controlled motor } \\
\text { shutdown }\end{array}$ & $\begin{array}{l}\text { Medium; alternative } \\
\text { sensor modality available }\end{array}$ & $\begin{array}{l}\text { Redundancy achieved by } \\
\text { employing both physical } \\
\text { and virtual sensors to } \\
\text { make system fault tolerant }\end{array}$ \\
\hline $\begin{array}{l}\text { Bearing } 1 \text { Virtual Position } \\
\text { Sensor }\end{array}$ & $\begin{array}{l}\text { Calculate X-Y shaft } \\
\text { position by interpreting } \\
\text { current phase and } \\
\text { amplitude in stator } \\
\text { windings }\end{array}$ & $\begin{array}{l}\text { Wrong centering position; } \\
\text { excessive uncertainty; loss } \\
\text { of signal }\end{array}$ & $\begin{array}{l}\text { Open winding; } \\
\text { interference from } \\
\text { electrical noise; shorted } \\
\text { windings }\end{array}$ & $\begin{array}{l}\text { Potential loss of position } \\
\text { control }\end{array}$ & $\begin{array}{l}\text { Signature analysis; model } \\
\text { comparison; comparison } \\
\text { with physical sensor }\end{array}$ & $\begin{array}{l}\text { Extraction of real-time } \\
\text { position information under } \\
\text { wide range of degraded } \\
\text { conditions }\end{array}$ & $\begin{array}{l}\text { Compare physical and } \\
\text { virtual position } \\
\text { measurements and } \\
\text { validate measurement }\end{array}$ & $\begin{array}{l}\text { Medium; alternative } \\
\text { sensor modality available }\end{array}$ & $\begin{array}{l}\text { Redundancy achieved by } \\
\text { employing both physical } \\
\text { and virtual sensors to } \\
\text { make system fault tolerant }\end{array}$ \\
\hline $\begin{array}{l}\text { Bearing1 Internal Rotor } \\
\text { Can }\end{array}$ & $\begin{array}{l}\text { Prevents salt from } \\
\text { attacking rotating bearing } \\
\text { magnetic components }\end{array}$ & $\begin{array}{l}\text { Leakage of salt into } \\
\text { bearing rotor }\end{array}$ & $\begin{array}{l}\text { Rubbing; pin-hole leaks; } \\
\text { weld failure }\end{array}$ & $\begin{array}{l}\text { Loss of magnetic function } \\
\text { from rotor can leakage }\end{array}$ & $\begin{array}{l}\text { Current signature; sensor } \\
\text { signal }\end{array}$ & $\begin{array}{l}\text { Diagnosis and prediction } \\
\text { of failing inner barrier }\end{array}$ & $\begin{array}{l}\text { Controlled motor } \\
\text { shutdown and repair }\end{array}$ & High; slow evolution & $\begin{array}{l}\text { Separate } \\
\text { compartmentalized } \\
\text { barriers; select salt } \\
\text { compatible materials; } \\
\text { employ multiple layers; an } \\
\text { eddy-current thickness } \\
\text { gauge is used to monitor } \\
\text { stationary can and infer } \\
\text { thickness degradation of } \\
\text { internal can }\end{array}$ \\
\hline $\begin{array}{l}\text { Liquid Salt Rotor Cooling } \\
\text { Circulation }\end{array}$ & $\begin{array}{l}\text { Maintains bearing rotor } \\
\text { temperature in normal } \\
\text { range }\end{array}$ & $\begin{array}{l}\text { Cooling flow is reduced or } \\
\text { stops }\end{array}$ & $\begin{array}{l}\text { Blockage of cooling } \\
\text { channel (partial or full); } \\
\text { loss of salt head pressure }\end{array}$ & $\begin{array}{l}\text { Temperature of bearing } \\
\text { rotor components exceeds } \\
\text { design limit and leads to } \\
\text { barrier failure and loss of } \\
\text { magnetic properties }\end{array}$ & $\begin{array}{l}\text { Thermocouples mounted } \\
\text { on outer surface of } \\
\text { stationary can }\end{array}$ & $\begin{array}{l}\text { Monitor for temperature } \\
\text { spikes or drift and } \\
\text { implement corrective } \\
\text { actions }\end{array}$ & $\begin{array}{l}\text { Scale back power level of } \\
\text { motor to reduce internal } \\
\text { heat generation }\end{array}$ & Medium; slow evolution & $\begin{array}{l}\text { Design rotor system with } \\
\text { multiple cooling channels }\end{array}$ \\
\hline $\begin{array}{l}\text { Bearing 1 Stator } \\
\text { Heating/Cooling }\end{array}$ & $\begin{array}{l}\text { Keeps stator above } \\
\text { minimum temperature to } \\
\text { prevent over-cooling of } \\
\text { salt inside stationary can } \\
\text { and removes heat above } \\
\text { specified temperature to } \\
\text { prevent overheating of } \\
\text { stator components }\end{array}$ & $\begin{array}{l}\text { Loss of coolant; loss of } \\
\text { coolant flow; reduced heat } \\
\text { removal; over-cooling }\end{array}$ & $\begin{array}{l}\text { Coolant leak; piping } \\
\text { rupture; TC failure; } \\
\text { coolant pump failure; } \\
\text { heater failure; loss of } \\
\text { power; loss of heat sink }\end{array}$ & $\begin{array}{l}\text { Stator windings exceed } \\
\text { maximum temperature } \\
\text { rating; magnetic materials } \\
\text { exceed thermal rating; salt } \\
\text { solidifies inside stationary } \\
\text { can }\end{array}$ & $\begin{array}{l}\text { Thermocouples mounted } \\
\text { in stator assembly monitor } \\
\text { temperature; coolant flow } \\
\text { is monitored; status of } \\
\text { coolant pump, heat sink, } \\
\text { and heaters is monitored }\end{array}$ & $\begin{array}{l}\text { PI controller is used to } \\
\text { (1) keep above minimum } \\
\text { temperature; (2) control } \\
\text { operating temperature to } \\
\text { set point. Diagnostics } \\
\text { identify failures and track } \\
\text { slowly changing } \\
\text { conditions. }\end{array}$ & $\begin{array}{l}\text { Temperatures above } \\
\text { threshold value for } \\
\text { specified period result in } \\
\text { motor power scale back to } \\
\text { reduce heat load. }\end{array}$ & Medium; slow evolution & $\begin{array}{l}\text { Secondary cooling system } \\
\text { can be employed either as } \\
\text { standby or parallel } \\
\text { simultaneous operation. } \\
\text { Redundant thermocouples. }\end{array}$ \\
\hline
\end{tabular}


Table A-17 (continued)

\begin{tabular}{|c|c|c|c|c|c|c|c|c|c|}
\hline Description & Function & Failure Mode & Cause of Failure & Effect of Failure & Detection Method & Control Requirement & Corrective Action & Severity & Defense in Depth \\
\hline $\begin{array}{l}\text { Bearing 1 Stator Power } \\
\text { Electronics }\end{array}$ & $\begin{array}{l}\text { Provide modulated current } \\
\text { to bearing stator windings }\end{array}$ & $\begin{array}{l}\text { Total loss of drive current; } \\
\text { loss of one or more } \\
\text { phases; limited current }\end{array}$ & $\begin{array}{l}\text { Blown fuse or circuit } \\
\text { breaker; Safety protective } \\
\text { feature engaged; failed } \\
\text { IGBT or other electronic } \\
\text { component; over-heating } \\
\text { (loss of electronics } \\
\text { cooling); interlock } \\
\text { protection activated } \\
\text { because of maintenance or } \\
\text { operating personnel }\end{array}$ & $\begin{array}{l}\text { Loss of position control; } \\
\text { bearing loses holding } \\
\text { force and rotor impacts } \\
\text { stationary can; limit cycle } \\
\text { oscillation starts; motor } \\
\text { failure }\end{array}$ & $\begin{array}{l}\text { Drive current is separately } \\
\text { monitored; status and } \\
\text { temperature of drive } \\
\text { electronics is monitored; } \\
\text { sensors watch rotor } \\
\text { position }\end{array}$ & $\begin{array}{l}\text { Current and voltage are } \\
\text { monitored to prevent } \\
\text { operation in damage } \\
\text { region of electronics and } \\
\text { to keep away from } \\
\text { protective features }\end{array}$ & $\begin{array}{l}\text { Secondary electronics } \\
\text { package seamlessly } \\
\text { activated to prevent loss } \\
\text { of position control }\end{array}$ & High; rapid evolution & $\begin{array}{l}\text { Secondary or tertiary } \\
\text { drive electronics required } \\
\text { to meet reliability goals; } \\
\text { possibly a second bearing } \\
\text { stator winding with } \\
\text { associated drive } \\
\text { electronics is incorporated }\end{array}$ \\
\hline $\begin{array}{l}\text { Bearing } 1 \text { Drive } \\
\text { Electronics Power Supply }\end{array}$ & $\begin{array}{l}\text { Provides dc voltage at } \\
\text { required current to the } \\
\text { drive electronics }\end{array}$ & $\begin{array}{l}\text { Loss of power; drop in } \\
\text { supply voltage; } \\
\text { intermittent voltage; noise } \\
\text { or oscillation in voltage }\end{array}$ & $\begin{array}{l}\text { Blown fuse or circuit } \\
\text { breaker; Safety protective } \\
\text { feature engaged; failed } \\
\text { component; over-heating; } \\
\text { interlock protection } \\
\text { activated }\end{array}$ & $\begin{array}{l}\text { Prevents stator drive } \\
\text { electronics from } \\
\text { operating; bearing ceases } \\
\text { functioning }\end{array}$ & $\begin{array}{l}\text { Power supply voltage } \\
\text { monitoring; power supply } \\
\text { status monitoring }\end{array}$ & $\begin{array}{l}\text { Diagnostics of power } \\
\text { supply parameters predicts } \\
\text { developing problems. }\end{array}$ & $\begin{array}{l}\text { Abrupt loss of power is } \\
\text { mitigated by having } \\
\text { redundant power supplies } \\
\text { or a secondary power } \\
\text { source used only for } \\
\text { coast-down during loss- } \\
\text { of-power conditions }\end{array}$ & High; rapid evolution & $\begin{array}{l}\text { Secondary power source } \\
\text { needed to meet reliability } \\
\text { goals; stored energy in } \\
\text { secondary source need } \\
\text { only meet controlled } \\
\text { shutdown needs }\end{array}$ \\
\hline $\begin{array}{l}\text { Bearing } 1 \text { Input } \\
\text { Electronics }\end{array}$ & $\begin{array}{l}\text { Receives signals from } \\
\text { sensors, current } \\
\text { transformers, voltage } \\
\text { measurements, } \\
\text { thermocouples, acoustic } \\
\text { probes; } \text { eddy current } \\
\text { probes }\end{array}$ & $\begin{array}{l}\text { Input not responding; } \\
\text { excessive noise in input; } \\
\text { drift or bias in signal } \\
\text { value; cross-talk between } \\
\text { sensor signals }\end{array}$ & $\begin{array}{l}\text { Lighting or electrostatic } \\
\text { discharge; ground loop } \\
\text { formed; failed input stage } \\
\text { component(s); A/D } \\
\text { converter failure; shorted } \\
\text { or open wiring } \\
\text { connections; corroded } \\
\text { connections; input } \\
\text { computer hacked }\end{array}$ & $\begin{array}{l}\text { Real-time motor } \\
\text { measurements are } \\
\text { corrupted or unavailable; } \\
\text { incorrect control action } \\
\text { may be taken }\end{array}$ & $\begin{array}{l}\text { Signal validation methods } \\
\text { used to identify both } \\
\text { sensor and input } \\
\text { electronics degradation } \\
\text { and failure }\end{array}$ & $\begin{array}{l}\text { Control should be } \\
\text { maintained over full or } \\
\text { degraded operation even } \\
\text { under loss of sensory } \\
\text { input conditions }\end{array}$ & $\begin{array}{l}\text { Alternative control } \\
\text { algorithms may be } \\
\text { employed for degraded or } \\
\text { missing sensor } \\
\text { information }\end{array}$ & $\begin{array}{l}\text { Low to high depending on } \\
\text { failure }\end{array}$ & $\begin{array}{l}\text { Multiple input stages } \\
\text { needed to provide } \\
\text { protection against single } \\
\text { failures }\end{array}$ \\
\hline $\begin{array}{l}\text { Bearing } 1 \text { Diagnostics and } \\
\text { Control Computer } \\
\text { Subsystem }\end{array}$ & $\begin{array}{l}\text { Processes signals to } \\
\text { accomplish control of } \\
\text { bearing functions under } \\
\text { all normal and abnormal } \\
\text { conditions, diagnoses } \\
\text { current signals and detects } \\
\text { developing problems, } \\
\text { receives commands, and } \\
\text { generates status } \\
\text { information }\end{array}$ & $\begin{array}{l}\text { Control not responding; } \\
\text { incorrect bearing position } \\
\text { or force signals generated; } \\
\text { erratic or noisy drive } \\
\text { signals generated }\end{array}$ & $\begin{array}{l}\text { Control computer failure; } \\
\text { incorrect set points or } \\
\text { algorithms applied; signal } \\
\text { or command validation } \\
\text { algorithm failed to detect } \\
\text { anomaly; control } \\
\text { computer hacked; output } \\
\text { validation subsystem } \\
\text { failed to detect anomalous } \\
\text { drive signals }\end{array}$ & $\begin{array}{l}\text { Bearing fails to center } \\
\text { rotor and shaft; motor } \\
\text { degrades or fails; rotor } \\
\text { impacts stationary can }\end{array}$ & $\begin{array}{l}\text { Watch dog subsystem } \\
\text { detects basic health and } \\
\text { functioning of control } \\
\text { computer hardware; } \\
\text { independent output } \\
\text { validation subsystem } \\
\text { determines whether drive } \\
\text { signal timing and } \\
\text { amplitude is within } \\
\text { specification }\end{array}$ & $\begin{array}{l}\text { Control system should } \\
\text { generate current output } \\
\text { values for bearing stator to } \\
\text { accomplish the } \\
\text { performance standard for } \\
\text { the system }\end{array}$ & $\begin{array}{l}\text { Control system should } \\
\text { continue operation } \\
\text { without incident under } \\
\text { any single failure; } \\
\text { continued operation } \\
\text { should extend to a wide } \\
\text { variety of degraded } \\
\text { component conditions; } \\
\text { control should degrade } \\
\text { gracefully under the worst } \\
\text { case component failures }\end{array}$ & $\begin{array}{l}\text { worst case is high; } \\
\text { degradation can progress } \\
\text { rapidly }\end{array}$ & $\begin{array}{l}\text { Ultimately double or triple } \\
\text { redundancy should be } \\
\text { employed to protect } \\
\text { against single failures; } \\
\text { resilient control strategies } \\
\text { should be incorporated to } \\
\text { provide a high degree of } \\
\text { protection against hacking }\end{array}$ \\
\hline
\end{tabular}


Table A-18. Drive rotor system components

\begin{tabular}{|c|c|c|c|c|c|c|c|c|c|}
\hline Description & Function & Failure Mode & Cause of Failure & Effect of Failure & Detection Method & Control Requirement & Corrective Action & Severity & Defense in Depth \\
\hline Drive Rotor & $\begin{array}{l}\text { Supply rotational torque to } \\
\text { shaft (ultimately to pump } \\
\text { load) }\end{array}$ & $\begin{array}{l}\text { Rub or impact outer } \\
\text { barrier stationary can; } \\
\text { detachment from shaft; } \\
\text { excessive eddy losses; } \\
\text { excessive vibration }\end{array}$ & $\begin{array}{l}\text { Excessive out-of-balance } \\
\text { force; corrosion; } \\
\text { manufacturing defect; } \\
\text { overheating; particle } \\
\text { lodged in gap region; } \\
\text { intralaminar insulation } \\
\text { breakdown; nonuniform } \\
\text { movement of laminations }\end{array}$ & $\begin{array}{l}\text { Motor failure; barrier } \\
\text { penetration; vibration that } \\
\text { overpowers holding force } \\
\text { of bearings }\end{array}$ & $\begin{array}{l}\text { Current signature analysis; } \\
\text { sensor signal; vibration } \\
\text { sensors }\end{array}$ & $\begin{array}{l}\text { Fast response; high } \\
\text { emergency drive } \\
\text { capability; asymmetric } \\
\text { drive capability for out-of- } \\
\text { balance compensation }\end{array}$ & $\begin{array}{l}\text { Rapid stop of motor if } \\
\text { warranted; speed } \\
\text { reductions asymmetric } \\
\text { polyphase drive }\end{array}$ & $\begin{array}{l}\text { Medium to high; rapid } \\
\text { evolution is possible }\end{array}$ & $\begin{array}{l}\text { Multiple barrier layers to } \\
\text { slow development of leak }\end{array}$ \\
\hline Drive Stator & $\begin{array}{l}\text { Circumferentially } \\
\text { sequence magnetic field } \\
\text { to apply torque to drive } \\
\text { rotor }\end{array}$ & Open winding & $\begin{array}{l}\text { Over current; over } \\
\text { temperature; voltage arc- } \\
\text { over; latent flaw; corrosion }\end{array}$ & $\begin{array}{l}\text { Motor failure; reduced } \\
\text { torque; vibration; reduced } \\
\text { speed; invoke degraded } \\
\text { mode operation }\end{array}$ & $\begin{array}{l}\text { Current monitoring, } \\
\text { voltage monitoring; } \\
\text { vibration sensors }\end{array}$ & $\begin{array}{l}\text { Diagnostics of current and } \\
\text { voltage signature must be } \\
\text { performed continuously }\end{array}$ & $\begin{array}{l}\text { None unless there is } \\
\text { redundant windings }\end{array}$ & High; rapid evolution & $\begin{array}{l}\text { Redundant or spare stator } \\
\text { windings; plan for } \\
\text { degraded operation with } \\
\text { loss of drive phase }\end{array}$ \\
\hline $\begin{array}{l}\text { Drive Rotor Rotational } \\
\text { Position Sensor }\end{array}$ & $\begin{array}{l}\text { Measure rotational } \\
\text { position of rotor (and } \\
\text { speed) for use by control } \\
\text { system to generate drive } \\
\text { current for each phase and } \\
\text { for diagnostics }\end{array}$ & $\begin{array}{l}\text { Wrong position indication; } \\
\text { intermittent or noisy } \\
\text { operation; dead sensor }\end{array}$ & $\begin{array}{l}\text { Over temperature; latent } \\
\text { flaw; excessive noise }\end{array}$ & $\begin{array}{l}\text { Loss of rotor-stator drive } \\
\text { synchronization; excessive } \\
\text { heat dissipation; loss of } \\
\text { speed control; reverse } \\
\text { direction possible }\end{array}$ & $\begin{array}{l}\text { Loss of sensor signal; } \\
\text { sensor signal not in bounds } \\
\text { of model prediction; signal } \\
\text { validation methods are } \\
\text { used to identify failing } \\
\text { sensors }\end{array}$ & $\begin{array}{l}\text { Continuous diagnostics of } \\
\text { sensor signal; alternative } \\
\text { means of determining } \\
\text { rotor rotational position }\end{array}$ & $\begin{array}{l}\text { Switch to alternative } \\
\text { position measurement } \\
\text { method; use model-based } \\
\text { prediction; plan for } \\
\text { controlled motor shutdown }\end{array}$ & $\begin{array}{l}\text { Medium; alternative } \\
\text { sensor modality available }\end{array}$ & $\begin{array}{l}\text { Redundancy achieved by } \\
\text { employing both physical } \\
\text { and virtual sensors to } \\
\text { make system fault tolerant }\end{array}$ \\
\hline $\begin{array}{l}\text { Drive Rotor Rotational } \\
\text { Virtual Position Sensor }\end{array}$ & $\begin{array}{l}\text { Calculate rotational } \\
\text { position (and speed) of } \\
\text { rotor for control system } \\
\text { use by interpreting current } \\
\text { and amplitude for each } \\
\text { stator winding phase }\end{array}$ & $\begin{array}{l}\text { Wrong interpretation of } \\
\text { phase information; } \\
\text { excessive uncertainty; loss } \\
\text { of signal }\end{array}$ & $\begin{array}{l}\text { Open winding; } \\
\text { interference from electrical } \\
\text { noise; shorted windings }\end{array}$ & $\begin{array}{l}\text { Loss of speed control; } \\
\text { lower torque; }\end{array}$ & $\begin{array}{l}\text { Signature analysis; model } \\
\text { comparison; comparison } \\
\text { with physical sensor }\end{array}$ & $\begin{array}{l}\text { Extraction of real-time } \\
\text { position information under } \\
\text { wide range of degraded } \\
\text { conditions }\end{array}$ & $\begin{array}{l}\text { Compare physical and } \\
\text { virtual position } \\
\text { measurements and validate } \\
\text { measurement }\end{array}$ & $\begin{array}{l}\text { Medium; alternative } \\
\text { sensor modality available }\end{array}$ & $\begin{array}{l}\text { Redundancy achieved by } \\
\text { employing both physical } \\
\text { and virtual sensors to } \\
\text { make system fault tolerant }\end{array}$ \\
\hline Drive Rotor Internal Can & $\begin{array}{l}\text { Prevents salt from } \\
\text { attacking drive rotor } \\
\text { magnetic components } \\
\text { (iron cobalt alloy), which } \\
\text { are quickly attacked by } \\
\text { liquid salt }\end{array}$ & Leakage of salt into rotor & $\begin{array}{l}\text { Rubbing; pin-hole leaks; } \\
\text { weld failure }\end{array}$ & $\begin{array}{l}\text { Loss of magnetic function } \\
\text { because material is } \\
\text { corroded away; } \\
\text { detachment of rotor } \\
\text { magnetics from shaft; } \\
\text { disintegration of rotor } \\
\text { assembly }\end{array}$ & $\begin{array}{l}\text { Current signature; sensor } \\
\text { signal; vibration }\end{array}$ & $\begin{array}{l}\text { Diagnosis and prediction } \\
\text { of rotor failure }\end{array}$ & $\begin{array}{l}\text { Controlled motor } \\
\text { shutdown and repair } \\
\text { before extensive damage }\end{array}$ & High; slow evolution & $\begin{array}{l}\text { Separate } \\
\text { compartmentalized } \\
\text { barriers can prevent } \\
\text { corrosion propagation; } \\
\text { select salt compatible } \\
\text { materials; employ multiple } \\
\text { layers; an eddy-current } \\
\text { thickness gauge } \\
\text { monitoring stationary can } \\
\text { and infer thickness } \\
\text { degradation of internal can }\end{array}$ \\
\hline
\end{tabular}


Table A-18 (continued)

\begin{tabular}{|c|c|c|c|c|c|c|c|c|c|}
\hline Description & Function & Failure Mode & Cause of Failure & Effect of Failure & Detection Method & Control Requirement & Corrective Action & Severity & Defense in Depth \\
\hline $\begin{array}{l}\text { Liquid Salt Drive Rotor } \\
\text { Cooling Circulation }\end{array}$ & $\begin{array}{l}\text { Maintains drive rotor } \\
\text { temperature in normal } \\
\text { range }\end{array}$ & $\begin{array}{l}\text { Cooling flow is reduced or } \\
\text { stops }\end{array}$ & \begin{tabular}{|l|} 
Blockage of cooling \\
channel (partial or full); \\
loss of salt head pressure; \\
vapor lock
\end{tabular} & \begin{tabular}{|l|} 
Impaired rotor cooling \\
resulting in temperature of \\
rotor magnetic and \\
insulation exceeding \\
design limit; can lead to \\
barrier failure and loss of \\
magnetic properties
\end{tabular} & $\begin{array}{l}\text { Thermocouples mounted } \\
\text { on outer surface of } \\
\text { stationary can; vibration } \\
\text { signature may identify } \\
\text { flow anomalies }\end{array}$ & $\begin{array}{l}\text { Monitor for temperature } \\
\text { spikes or drift and } \\
\text { implement corrective } \\
\text { actions }\end{array}$ & $\begin{array}{l}\text { Scale back power level of } \\
\text { motor to reduce internal } \\
\text { heat generation as a } \\
\text { temporary solution }\end{array}$ & Medium; slow evolution & $\begin{array}{l}\text { Design rotor system with } \\
\text { multiple cooling channels }\end{array}$ \\
\hline $\begin{array}{l}\text { Drive Stator } \\
\text { Heating/Cooling }\end{array}$ & $\begin{array}{l}\text { Keeps drive stator above } \\
\text { minimum temperature to } \\
\text { prevent over-coling of } \\
\text { salt inside stationary can } \\
\text { and removes heat above } \\
\text { specified temperature to } \\
\text { prevent overheating of } \\
\text { stator components (e.g., } \\
\text { wire) }\end{array}$ & $\begin{array}{l}\text { Loss of coolant; loss of } \\
\text { coolant flow; reduced heat } \\
\text { removal; over-cooling }\end{array}$ & $\begin{array}{l}\text { Coolant leak; piping } \\
\text { ruppture; TC failure; } \\
\text { coolant pump failure; } \\
\text { heater failure; loss of } \\
\text { power; loss of heat sink; } \\
\text { control system failure }\end{array}$ & \begin{tabular}{|l|} 
Stator windings exceed \\
maximum temperature \\
rating; magnetic materials \\
exceed thermal rating; salt \\
solidifies inside stationary \\
can
\end{tabular} & $\begin{array}{l}\text { Thermocouples mounted } \\
\text { in stator assembly monitor } \\
\text { temperature; coolant flow } \\
\text { is monitored; status of } \\
\text { coolant pump, heat sink, } \\
\text { and heaters is monitored }\end{array}$ & $\begin{array}{l}\text { PI controller is used to } \\
\text { (1) keep stator above } \\
\text { minimum temperature; } \\
\text { (2) control operating } \\
\text { temperature to set point. } \\
\text { Diagnostics identify } \\
\text { failures and track changing } \\
\text { conditions. }\end{array}$ & $\begin{array}{l}\text { Temperatures above } \\
\text { threshold value for } \\
\text { specified period result in } \\
\text { motor power scale back to } \\
\text { reduce heat load. }\end{array}$ & Medium; slow evolution & $\begin{array}{l}\text { Secondary cooling system } \\
\text { can be employed either as } \\
\text { standby or parallel } \\
\text { simultaneous operation. } \\
\text { Redundant thermocouples. }\end{array}$ \\
\hline $\begin{array}{l}\text { Drive Stator Power } \\
\text { Electronics }\end{array}$ & $\begin{array}{l}\text { Provide modulated current } \\
\text { to poly-phase stator } \\
\text { windings }\end{array}$ & $\begin{array}{l}\text { Total loss of drive current; } \\
\text { loss of one or more } \\
\text { phases; current limiting } \\
\text { invoked }\end{array}$ & \begin{tabular}{|l|} 
Blown fuse or circuit \\
breaker; safety protective \\
feature engaged; failed \\
IGBT or other electronic \\
component; over-heating \\
(loss of electronics \\
cooling); interlock \\
protection activated \\
because of maintenance or \\
operating personnel
\end{tabular} & $\begin{array}{l}\text { Motor failure; reduced } \\
\text { torque; vibration; reduced } \\
\text { speed; invoke degraded } \\
\text { mode operation; drive } \\
\text { imbalance causes rotor to } \\
\text { impact stationary can; } \\
\text { limit cycle oscillation } \\
\text { starts }\end{array}$ & $\begin{array}{l}\text { Drive current is separately } \\
\text { monitored; status and } \\
\text { temperature of drive } \\
\text { electronics is monitored; } \\
\text { sensors watch rotor } \\
\text { rotation position and speed }\end{array}$ & $\begin{array}{l}\text { Current and voltage are } \\
\text { monitored to prevent } \\
\text { operation in damage } \\
\text { region of electronics and } \\
\text { to keep away from } \\
\text { protective features }\end{array}$ & $\begin{array}{l}\text { Secondary electronics } \\
\text { package seamlessly } \\
\text { activated to prevent loss of } \\
\text { position control }\end{array}$ & High; rapid evolution & $\begin{array}{l}\text { Secondary (or tertiary) } \\
\text { drive electronics required } \\
\text { to meet reliability goals; } \\
\text { possibly a second drive } \\
\text { stator winding with } \\
\text { associated drive } \\
\text { electronics is incorporated }\end{array}$ \\
\hline $\begin{array}{l}\text { Main Drive Electronics } \\
\text { Power Supply }\end{array}$ & $\begin{array}{l}\text { Provides dc voltage at } \\
\text { required current to the } \\
\text { drive electronics }\end{array}$ & $\begin{array}{l}\text { Loss of power; drop in } \\
\text { supply voltage; } \\
\text { intermittent voltage; noise } \\
\text { or oscillation in voltage }\end{array}$ & \begin{tabular}{|l|} 
Blown fuse or circuit \\
breaker; Safety protective \\
feature engaged; failed \\
component; over-heating; \\
interlock protection \\
activated
\end{tabular} & $\begin{array}{l}\text { Prevents stator drive } \\
\text { electronics from operating; } \\
\text { motor ceases functioning }\end{array}$ & \begin{tabular}{|l|} 
Power supply voltage \\
monitoring; power supply \\
status monitoring
\end{tabular} & $\begin{array}{l}\text { Diagnostics of power } \\
\text { supply parameters predicts } \\
\text { developing problems. }\end{array}$ & $\begin{array}{l}\text { Loss of power is mitigated } \\
\text { by having redundant } \\
\text { power supplies or a } \\
\text { secondary power source }\end{array}$ & High; rapid evolution & $\begin{array}{l}\text { Secondary power source } \\
\text { may be needed to meet } \\
\text { reliability goals }\end{array}$ \\
\hline
\end{tabular}


Table A-18 (continued)

\begin{tabular}{|c|c|c|c|c|c|c|c|c|c|}
\hline Description & Function & Failure Mode & Cause of Failure & Effect of Failure & Detection Method & Control Requirement & Corrective Action & Severity & Defense in Depth \\
\hline $\begin{array}{l}\text { Main Drive Input } \\
\text { Electronics }\end{array}$ & $\begin{array}{l}\text { Receives signals from } \\
\text { sensors, current } \\
\text { transformers, voltage } \\
\text { measurements, } \\
\text { thermocouples, acoustic } \\
\text { probes; eddy current } \\
\text { probes }\end{array}$ & $\begin{array}{l}\text { Input not responding; } \\
\text { excessive noise in input; } \\
\text { drift or bias in signal } \\
\text { value; cross-talk between } \\
\text { sensor signals }\end{array}$ & $\begin{array}{l}\text { Lighting or electrostatic } \\
\text { discharge; ground loop } \\
\text { formed; failed input stage } \\
\text { component(s); } \mathrm{A} / \mathrm{D} \\
\text { converter failure; shorted } \\
\text { or open wiring } \\
\text { connections; corroded } \\
\text { connections; input } \\
\text { computer hacked }\end{array}$ & $\begin{array}{l}\text { Real-time motor } \\
\text { measurements are } \\
\text { corrupted or unavailable; } \\
\text { incorrect control action } \\
\text { may be taken; motor } \\
\text { ceases operation }\end{array}$ & $\begin{array}{l}\text { Signal validation methods } \\
\text { used to identify both } \\
\text { sensor and input } \\
\text { electronics degradation } \\
\text { and failure }\end{array}$ & $\begin{array}{l}\text { Control should be } \\
\text { maintained over full or } \\
\text { degraded operation even } \\
\text { under loss of sensory input } \\
\text { conditions }\end{array}$ & $\begin{array}{l}\text { Alternative control } \\
\text { algorithms may be } \\
\text { employed for degraded or } \\
\text { missing sensor information }\end{array}$ & $\begin{array}{l}\text { Low to high depending on } \\
\text { failure }\end{array}$ & $\begin{array}{l}\text { Multiple input stages } \\
\text { needed to provide } \\
\text { protection against single } \\
\text { failures }\end{array}$ \\
\hline $\begin{array}{l}\text { Main Drive Diagnostics } \\
\text { and Control Computer } \\
\text { Subsystem }\end{array}$ & $\begin{array}{l}\text { Processes signals to } \\
\text { accomplish control of } \\
\text { rotor functions under all } \\
\text { normal and abnormal } \\
\text { conditions, diagnoses } \\
\text { current signals and detects } \\
\text { developing problems, } \\
\text { receives commands, and } \\
\text { generates status } \\
\text { information }\end{array}$ & $\begin{array}{l}\text { Control not responding; } \\
\text { incorrect drive current } \\
\text { sequence generated } \\
\text { including phase reversal; } \\
\text { erratic or noisy drive } \\
\text { signals generated }\end{array}$ & $\begin{array}{l}\text { Control computer failure; } \\
\text { incorrect set points or } \\
\text { algorithms applied, signal } \\
\text { or command validation } \\
\text { algorithm failed to detect } \\
\text { anomaly; control computer } \\
\text { hacked; output validation } \\
\text { subsystem failed to detect } \\
\text { anomalous drive signals }\end{array}$ & $\begin{array}{l}\text { Motor failure; reduced } \\
\text { torque; vibration; reduced } \\
\text { speed; direction reversal; } \\
\text { invoked degraded mode } \\
\text { operation; drive imbalance } \\
\text { causes rotor to impact } \\
\text { stationary can; limit cycle } \\
\text { oscillation starts }\end{array}$ & $\begin{array}{l}\text { Watch dog subsystem } \\
\text { detects basic health and } \\
\text { functioning of control } \\
\text { computer hardware; } \\
\text { independent output } \\
\text { validation subsystem } \\
\text { determines whether drive } \\
\text { signal timing and } \\
\text { amplitude is within } \\
\text { specification }\end{array}$ & $\begin{array}{l}\text { Control system should } \\
\text { generate current output } \\
\text { values for drive stator } \\
\text { individual phases to } \\
\text { accomplish the system } \\
\text { performance standard }\end{array}$ & $\begin{array}{l}\text { Control system should } \\
\text { continue operation without } \\
\text { incident under any single } \\
\text { failure; continued } \\
\text { operation should extend to } \\
\text { a wide variety of degraded } \\
\text { component conditions; } \\
\text { control should degrade } \\
\text { gracefully under the worst } \\
\text { case component failures }\end{array}$ & $\begin{array}{l}\text { worst case failure is high } \\
\text { severity; degradation can } \\
\text { progress rapidly }\end{array}$ & $\begin{array}{l}\text { Ultimately double or triple } \\
\text { redundancy should be } \\
\text { employed to protect } \\
\text { against single failures; } \\
\text { resilient control strategies } \\
\text { should be incorporated to } \\
\text { provide a high degree of } \\
\text { protection against hacking }\end{array}$ \\
\hline
\end{tabular}


